\title{
Assessment of myocardial injury : analysis of simultaneously sampled plasma activities of several cardiac enzymes
}

Citation for published version (APA):

Willems, G. M. (1985). Assessment of myocardial injury : analysis of simultaneously sampled plasma activities of several cardiac enzymes. [Doctoral Thesis, Maastricht University]. Rijksuniversiteit Limburg. https://doi.org/10.26481/dis.19851018gw

Document status and date:

Published: 01/01/1985

DOI:

10.26481/dis.19851018gw

Document Version:

Publisher's PDF, also known as Version of record

Please check the document version of this publication:

- A submitted manuscript is the version of the article upon submission and before peer-review. There can be important differences between the submitted version and the official published version of record.

People interested in the research are advised to contact the author for the final version of the publication, or visit the DOI to the publisher's website.

- The final author version and the galley proof are versions of the publication after peer review.

- The final published version features the final layout of the paper including the volume, issue and page numbers.

Link to publication

\footnotetext{
General rights rights.

- You may freely distribute the URL identifying the publication in the public portal. please follow below link for the End User Agreement:

www.umlib.nl/taverne-license

Take down policy

If you believe that this document breaches copyright please contact us at:

repository@maastrichtuniversity.nl

providing details and we will investigate your claim.
}

Copyright and moral rights for the publications made accessible in the public portal are retained by the authors and/or other copyright owners and it is a condition of accessing publications that users recognise and abide by the legal requirements associated with these

- Users may download and print one copy of any publication from the public portal for the purpose of private study or research.

- You may not further distribute the material or use it for any profit-making activity or commercial gain

If the publication is distributed under the terms of Article $25 \mathrm{fa}$ of the Dutch Copyright Act, indicated by the "Taverne" license above, 
ASSESSMENT OF MYOCARDIAL INJURY.

\begin{abstract}
ANALYSIS OF SIMULTANEOUSLY
SAMPLED PLASMA ACTIVITIES

OF SEVERAL CARDIAC ENZTMES
\end{abstract}

Proefschrift

ter verkrijging van de graad van doctor in de geneeskunde aan de Rijksuniversiteit Limburg te Maastricht, op gezag van de Rector Magnificus, Prof. Dr. F.I.M. Bonke, volgens het besluit van het college van Dekanen, in het openbaar te verdedigen op vrijdag 18 oktober 1985, om 16.00 wur.

$$
\text { door }
$$

George Marcel Willems

geboren te vtrecht in 1948 
Promotor

- Prof. Dr. H.C. Henker, Rijksuniversiteit Limburg, Mastricht

Co-promotor : Dr. W. Th. Hermens, Rijksuniversiteit Limburg, Mastricht

Refecenten : Prof Dr. P. Eyckhoff, Technische Hogeschool Eindhoven, Einahoven

Dr. A. van der Laarse, Academisch ziekenhuis Leiden, Leiden

Prof. Dr. H.J.J. Wellens, Rijksuniversiteit Limburg, Mastricht 
Aan mijn Moeder 
CONTENTS

CHAPTER 1 : INTRODUCTION

1.1 : Plasma levels of tissue enzymes as indicators of cardiac damage

1.2 : Quantification of cumulative enzyme release into plasma

1.3: The present study

CHAPTER 2 : TURNOVER AND DISTRIBUTION OF CIRCULATING PROTEINS

2.1 : Introduction

2.2 : Turnover and distribution of circulating proteins

2.3 : Kinetic studies: Models

2.4: Kinetic studies: Plasma proteins

2.5 : Kinetic studies: Tissue enzymes

2.6 : Entrance into plasma of tissue enzymes

APPENDIX A : DERTVATION OF THE MATHEMATICAL EXPRESSIONS USED IN CHAPTER 2

A1 : Compartmental analysis

A2 : The two-compartment model

A3 : The three-compartment mamilary model

A4 : Quantification of the input into plasma

CHAPTER 3 : ANALYSIS OF PLASMA ACTIVITIES OF SEVERAL SIMULTANEOUSLY SAMPLED ENZYMES

3.1 : Introduction

3.2 : Estimation of the circulatory parameters from simultaneously sampled plasma activities

3.3 : The estimation procedure

3.4: The performance of the estimation procedure

3.5 : Departures from the model

3.6. Simplified estimation procedures 
APPENDIX B : DERIVATION OF THE MATHEMATICAL EXPRESSIONS USED IN CHAPTER 3

Bl : Identifiability of the unit impulse response from simultaneously sampled plasma activities.

B2 : The numerical implementation of the estimation procedure

B3 : Statistical analysis

B4 : The calculation of simulated data

CHAPTER 4 : ESTIMATION OF THE CIRCULATORY PARAMETERS OF CARDIAC ENZYMES IN PATIENTS WITH AMI

4.1 : Introduction

4.2 : Methods

4.3 : Results

4.4 : Discussion

CHAPTER 5 : ENZYMATIC ASSESSMENT OF INJURY AFTER HEART SURGERY

5.1 : Introduction

5.2 : Methods

5.3 : Results

5.4 : Discussion

REFERENCES

CONCLUSIONS and SUMMARY

ABBREVIATIONS

CURRICULUM VITAE 


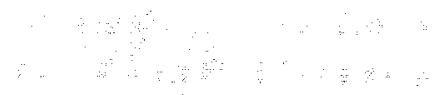


CHAPTER 1

TNTRODUCTION

1.1 Plasma levels of tissue enzymes as indicators of cardiac damage

The rise of plasma levels of tissue enzymes as a conseguence of tissue injury was already discovered in 1908, when wohlgemuth developed a sensitive assay for the detection of amylase activity and showed a rise of this activity in the plasma of patients with acute pancreatitis [Wohlgemuth, 1908].

For the heart the development of clinical enzymology started in 1954 with the observation of LaDue that acute myocardial infarction (AMI) in man was followed by a transient elevation of the plasma activity of aspartate aminotransferase (AST) (LaDue, 1954]. This report triggered an active interest in the study of enzyme release into plasma after tissue injury. In the following years there emerged much evidence that after AMI essentially any enzyme that is present in an appreciable amount in the cytosol of cardiac myocytes leakes from the infarcted area into the bloodstream; for a review see [Trautshold, 1968, Kupper, 1979]. Thus it became clear that elevated plasma levels of e.g. AST, aldolase (ALD) and lactate dehydrogenase (LDH) present sensitive markers of cardiac damage [Agress, 1960]. As these enzymes are also abundantly present in a variety of other tissues such enzyme tests lacked specificity.

Soon this was recognized and the search for cardiospecific enzymes was incited, resulting in the introduction of heat stable lactate dehydrogenase [Strandjord, 1961] and alpha-hydroxybutyrate dehydrogenase (HBD) [Rosalki, 1963] both allowing the discrimination between cardiac enzyme release on the one hand and liver or muscle damage on the other hand. Improved specificity was also obtained by the introduction of creatine kinase (CK) as a muscle specific enzyme [Dreyfuss, 1960] and its cardiospecific iso-enzyme CK-MB [v.a. Veen, 1966]. 
In the same time much evidence became available substantiating that elevated plasma enzyme levels of cardiac enzymes are caused by leakage from cardiac tissue. In experimental infarctions it was found that 24 hours after coronary 1 igation AST is depleted from the central zone of infacction [Mydick, 1955; Agress, 1955] and that shortly after coronary ligation enzyme levels in cardiac Iymphe are increased [Dunn, 1958]. While the absence of elevated plasma levels of cardiac enzymes in patients with coronary insufficlency not resulting in myocardial infarction was indicative for a relation between enzyme leakage and tissue necrosis UVincent, 1965; Nissen, 1965]. Furthermore in experimental infarctions in dogs a correlation was demonstrated between the extent of the infarcted area and the resulting plasma activity elevations [Nydick, 1955; Agress, 1955; Ruegsegger, 1959]. In these early attempts the histological infarct size was assessed by inspection of a sectioned heart and was correlated to maximal plasma levels of enzyme activity. Though both are crude measures a significant (but rather low) correlation was found. This quantitative relation between the extent of tissue damage and plasma enzyme levels was substantiated by the observation in man that histological infarct size, mortality rate as well as incidence of heart failure are correlated to post infarction plasma enzyme levels in patients with AMI [Rosalki, 1963; Kibe, 1967; Smith, 19671 .

These findings combined with the concept that heart cells do not regenerate, i.e. necrosis of heart tissue is cumulative, have inspired attempts to estimate this cumulative myocardial damage from the observed plasma time-activity curves of cardiac enzymes. 


\section{2 Quantification of cumulative enzyme release into plasma}

Although in the earlier attempts mentioned above the authors used peak levels of plasma activity as a measure of the total amount of enzyme released, it was soon recognized that plasma levels of such a protein reflect a dynamic equilibrium between entrance into the plasma and removal from the plasma by elimination and redistribution to extravascular fluid such as lymphe [Dunn, 1958; Strandjord, 1961; Brüdigan, 1960]. These authors also explored the relation between the plasma disappearance curve of the same enzyme upon a bolus injection and the plasma levels observed after AMI. Thus it became evident that peak levels reached, for a certain amount released into plasma, are sensitive to the rate of input in plasma. This influence of the rate of release upon the maximal values reached in plasma especially invalidates the use of peak values in intervention studies, because a modified time-course of release and an effect on the extent of myocardial damage both are apt to occur [Hermens, 1975].

Apart from these practical arguments the exact quantification of the amount of enzyme entering the bloodstream is desirable because only then a quantity is obtained, which allows comparison of the amounts of different enzymes released into plasma, or comparison of the amount of enzyme entering plasma to the original enzyme content of the damaged tissue.

A method for the quantification of cumulative enzyme release from the observed plasma time-activity curve in patients with AMI was introduced in 1970 [Witteveen, 1970]. The model accounted for the elimination of enzyme from plasma and in a subsequent improved version [Witteveen, 1972] also for the redistribution of enzyme to an extravascular pool. It was recognized that the calculated quantities were predominantly determined by the rate of elimination assumed. Consequently an attempt was made to estimate the rate constant of elimination for the five enzymes considered in these studies [Hemker, 1972] from the plasma decay curves in the same patients. Infarct size was expressed in terms of grams of tissue lost by conversion of total enzyme release to gram equivalents by determination of heart tissue enzyme content. 
At that time in an experimental study in the dog on $c k$ a $h i g h$ correlation ( $r=0.96)$ was demonstrated between infarct size 24 hours after coronary ligation, defined by the enzyme depletion from the heart, and total enzyme release, as calculated from the plasma levels. The proposed method of quantification was based on analysis of the plasma disappearance curves observed after a bolus injection of a purified CK preparation [She11, 1971]. The model used only accounted for dilution of protein in the circulation and for elimination. Although initially an erroneously high distribution volume of 11 to of the bodyweight was used, which was subsequently corrected [Roberts, 1975], the method after this correction is essentially considered sound [Visser, 1981a]. However the recovery into plasma, i.e. the fraction of the amount of enzyme lost from myocardium that has entered the circulation, was found to be $0.15 \pm 0.05$ (mean \pm standard deviation). This small recovery was inputed to local inactivation.

Obviously such a process preventing 85 of the total enzyme loss from the heart to reach the circulation theoretically presents serious hazards for the validity of the total amount of enzyme that appears in the circulation as a measure of infarct size. Even small variations in the extent of local inactivation would severely affect the estimations.

Thus early in the seventies the two main issues in the field of enzymatic infarct sizing already had emerged. First, in order to calculate cumulative enzyme release a suitable model of the behaviour of the enzyme in the circulation must be selected and this model must be supplied with some rate constants, e.g. the rate constant for the elimination, that turn out to be rather variable depending on species and enzyme considered. In contrast to the situation in experimental animals the identification of the model and these parameters in man can not be performed by injection or infusion of (purified) enzyme preparations as this is considered to present a immunological $r$ isk.

The second issue regards the question of the validity of cumulative enzyme release as a measure for the extent of myocardial damage. This second point hinges mainly on the recovery in plasma of enzymes depleted from the heart. 
In view of the widespread use of enzymatic assessment of cardiac damage in patient after AMI and cardiac surgery (CS), the first point has up to now attracted remarkable 1 ltte attention. Nearly all studies in man use a simple one-compartment model, incorporating only entrance of enzyme into plasma and the limination from the plasma and neglecting extravasation to (and return from) the extravascular pool. The rate of elimination is estimated from the rate of decay of the downslope part of the plasma activity curve. The so determined apparent disappearance rate $k_{\mathrm{d}}$ only reflects the true elimination rate if enzyme release into plasma is terminated. The validity of this assumption is questionable [Brüdigan, 1960; slutsky, 1977].

In experimental studies in the dog on $\mathrm{LDH}$, AST, and CK the $k$ a so determined is by a factor 3 lower than the elimination rate observed after injection of enzyme preparations 『strandjord, 1961; Dunn, 1958; She11, 19711. Apparently the method is also rather sensitive to the selection of the time interval for the determination of the apparent disappearance rate as in patients reported values for $\mathrm{CK}$ range from $0.035\left(\mathrm{~h}^{-1}\right)$ [Grande, 1982] to $0.066\left(\mathrm{~h}^{-1}\right)$ [Norris, 1975].

With respect to the validity of enzymatic assessment of cardiac damage in patients with AMI a number of reassuring results have been reported. A correlation of $r=0.79$ was reported between the peak value of heat stable LDH and infarct size determined by nitroblue tetrazolium (NBT) staining in postmortem studied hearts [Erhardt, 1974]. An even better correlation $(x=$ 0.96) was found for the relation of cumulative CK release and postmortem infarct-size determined by the NBT test [Bleifeld, 1977]. The relation between total CK-MB recovery into plasma compared to $\mathrm{CK}-\mathrm{MB}$ depletion from the heart and to infarct size determined by the NBT-test was studied in a group of 22 patients with AMI which survived at least 48 hours but died before 17 days after onset of symptoms. Here also a relation was demonstrated between CK-MB and total CK release into plasma and infarct size by NBT staining [Grande, 1982]. Recently this result was confirmed in a multi-center study [Hackel, 1984]. 
These results tend to diverge from the results of experimental studies in the dog. In the above mentioned study [She11, 1971] the recovery although very low was remarkably constant $0.15 \pm$ 0.05 (mean \pm standard deviation). This contrasts to later studies using the same method but reporting a dependence on the method of producing ischemia and on the size of the infarction [Cairns, [978]. 


\subsection{The present study}

As mentioned before the identification of circulatory parameters of tissue enzymes, such as the rate of elimination, in man is hampered by the inadmissibility of injection or infusion of (purified) preparations of these enzymes. Hence $1 t$ would be of importance to be able to estimate these parameters from plasma curves observed in patients with a form of acute tissue damage accompanied by a transient release of these enzymes. As mentioned earlier the apparent disappearance rate of the plasma curve not necessarily reflects true elimination from plasma, because continuing release will lead to a underestimation. For protelns that are eliminated rapidly compared to the time scale of the release into plasma the period of continuing release cannot be estimated

(u) 1 )

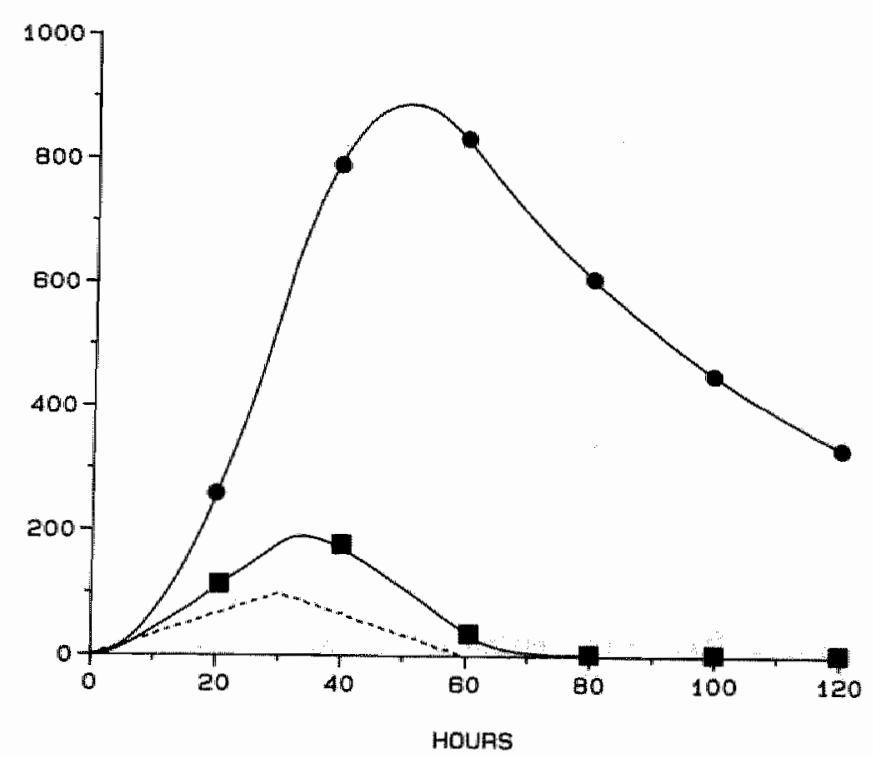

Fig. 1.1 Plasma activity curves calculated for the indicated trinagular meLease funotion (dabhed line; maximal value is $43 \mathrm{U} / 2 / \mathrm{h}$ ) for two valuee of the Fractional Catabolio Rate constant (FCR).

Rapia elimination ( $\mathbf{0}): F C R=0.20 \mathrm{~h}^{-1}$

stow elimination $(\bullet): F C R=0.015 \mathrm{~h}^{-1}$

Extravasation of enzyme is neglected. 
from the plasma curve as is illustrated in Fig. 1.1. With lacking knowledge about the exact moment of cessation of input into plasma the small time delay between termination of the release and the renormalisation of the plasma activities leaves no room for a safety margin. This contrasts to the situation with a slowly disappearing enzyme (Fig. 1.1).

This thesis deals with the development of a method for the estimation of the circulatory parameters from the plasma curves during continuing release of enzyme. Hereto we analyse the plasma curves of two or more different proteins simultaneously released into the bloodstream. By consideration of more plasma curves at the same time it turns out to be possible to estimate the input into plasma together with the circulatory parameters characterizing the behaviour of protein levels in plasma.

The approach followed requires that the proteins are released simultaneously into plasma and that the system response upon input in the circulation is linear, i.e. the response to the sum of two inputs equals the sum of the responses to the individual inputs. Although the underlying mechanisms causing the specific response of the system are in principle irrelevant for the estimation, it would be unwise to neglect the existing large body of knowledge on the behaviour of proteins in the circulation. Most data in this field are obtained in studies with radio labeled plasma proteins; an approach with a higher accuracy and more observables, such as the time course of the whole body content of injected protein, than obtainable for tissue enzymes. These data are reviewed in Chapter 2.

In Chapter 3 our method of estimation is introduced and a description is given of the algorithm used in the calculations. The performance of the procedure is assessed by application on simulated data sets.

The plasma activity curves of $\mathrm{CK}$, $\mathrm{HBD}$, and other cardiac enzymes sampled in patients after AMI are analyzed in chapter 4. with the estimates of the elimination rates thus obtained we reconsider the autopsy studies mentioned in the previous section. A further application is described in Chapter 5 where the enzyme release is compared in patients after AMI and after cardiac surgery. 
CHAPTER 2

TURNOVER AND DISTRIBUTION OF CIRCULATING PROTEINS

\section{I Introduction}

A quantitative interpretation of the dynamic state of plasma levels of a protein invariably requires the estimation of the amounts of protein entering and leaving the circulation. protein, once arrived in the plasma, is ultimately removed from the circulation, as is commonly seen after infusions in deficient subjects, after infusion of radiolabelled protein and after passive immunisation. However, the declining plasma levels observed in such cases not only reflect irreversible elimination but also a reversible redistribution of the protein to extravascular fluid compartments. An illustrative example of this redistribution is furnished by the rebound of the plasma level after a sudden depletion of the plasma pool observed when the plasma level of antibody is suddenly reduced by exchange transfusion or by injection of antigen [Gitlin, 1957].

The physiological data on the processes of removal of protein from the circulation and the exchange between the vascular and extravascular pool are discussed in section 2 of this chapter. Current knowledge of these processes is however insufficient to get reliable estimates of the rate of removal from and the rate of entrance into the plasma in the intact organism. Especialily the elimination of protein is poorly understood. The observed rates of elimination of different proteins show for instance variations of two orders of magnitude unexplained by the molecular composition.

In the fifties questions with respect to the rate of turnover and the size of the whole body pool of plasma proteins, combined with the availability of radioactive labels, resulted in kinetic studies with radiolabelled plasma proteins. This incited the development of compartmental models for the interpretation of the observed plasma disappearance curves of the injected label. A variety of formalisms and models is currently used. In Section 3 
of this chapter an attempt towards unification is made by taking the viewpoint that models should allow estimation of the eliminated quantities of protein and the size of the extravascular pool from the observables. The use of radiolabelled proteins not only allows a high accuracy in the determinations but also offers the possibility to estimate independently the instantaneous rate of elimination and (thus) of the size of the whole body pool. Another definite advantage is that only tracer amounts of labelled protein have to be injected which minimizes the perturbation of the system and obviates the complexities of non-linearity.

The highest quality data on the behaviour of macromolecules in the circulation curcently available were thus obtained in such studies. In section 4 these experiments with plasma proteins and the resulting circulatory model and parameters are reviewed.

Regrettably a comparable set of data on the dynamics of tissue enzymes in the body is lacking. Partly this is caused by the difficulties encountered in kinetic studies with tissue enzymes. These proteins appear to be very sensitive to molecular alterations in the process of isolation and purification, resulting in anomalous disappearance curves. Kinetic studies on the plasma disappearance of injected enzymes also have the disadvantage that the dose retained in the total body is not observable. From the data discussed in section 5 it is apparent that the absence of this check easily introduces misinterpretations. Another factor is the hesitation to inject tissue preparations in man.

Quantification of the amount of protein entered in the circulation from the observed plasma levels requires an estimation of the quantity of protein eliminated from the body and of the quantity still present in the extravascular compartment. These estimations are much simpler if the rates of the transport processes involved are linearly dependent on the pool sizes, $1 . e$. in case of first order rate transport processes. This linearity is also essential for the validity of most methods for the analysis of the kinetics of tissue enzymes.

For tissue enzymes this question received little attention in the literature. The results in this respect obtained for plasma proteins indicate that elimination rates depending non-linearly 
upon the plasma concentration do indeed occur, for instance for albumin. It is, however, questionable whether these situations are comparable as the level of albumin normally equals $40 \mathrm{gr} / \mathrm{L}$ whereas plasma concentrations of tissue enzymes are in the range of $\mathrm{mg} / 1$.

Synthesis of most plasma proteins is localized in the hepatocytes and the proteins are delivered directly to the plasma pool. Tissue proteins on the other hand normally are confined to the cell and only low levels are found in the plasma. The transient rise in plasma after injury is caused by leakage of these enzymes from cells in the damaged organ into the interstitial fluid and the subsequent transport to the plasma. In the last section of this chapter is devoted to the data on cellular enzyme release and on the pathways by which the enzymes once released gain access to the circulation. 


\section{2 Purnover and distrubution of circulating proteins}

\section{Elimination}

Surprisingly the process of removal of protein from the circu. lation is best understood for proteins that are damaged in some sense. For instance in the case of desialized glycoproteins ont has acquired the completeness of comprehension aimed at for native protein degradation. Upon treatment with neuramidase glycoprotein as ceruplasmin looses its sialic acid residue. In contrast to their native counterparts the asialic proteins disappear very rapialy from the circulation. The site of removal was identified by the finding that most of the radiolabelled protein, disappeared from the plasma, could be retrieved in the liver and that the primary site of binding was the hepatocyte membrane. This finding was corroborated by the subsequent demonstration that the carbohydrate residues exposed by the desialisation served as a recognition marker binding to a receptor in the hepatocyte membrane and that the rate of removal of various desialized glycoproteins correlated with the strength of binding [Ashwe11, 1974].

For denatured albumin, immunocomplexes and many complexes of proteins with specific inhibitory proteins it was demonstrated that removal from plasma largely takes place in the Kupfer cells in the liver [Schultze, 1966; Freeman, 1958; Hebert, 1976].

In contrast to these examples available evidence concerning the elimination of native proteins from the circulation does not allow an equivocal conclusion. Obvious pathways, in analogy to the elimination of drugs, the kidneys and the liver (also suggested by the previous examples) have received much attention, but with essentially negative results. Only for small proteins, as amylase, myoglobin and lysozyme that can pass the glomular membrane, it is demonstrated that clearance by urinary excretion takes place when the maximum capacity for reabsorption is exceeded [Ha]1, 1979]. For larger tissue enzymes it was demonstrated in experimental animals that integral removal of the kidneys, liver or spleen nor severe hemodynamic disturbances affect the 
rate of disappearance of injected enzymes IDumn, 1958 , strandjord, 1959, Fleisher, 1963a, Sibley, 1958, noberts, 19751. For albumin, the best studied plasma protein with respect to elimination, the contribution of liver and kidneys to the removal is considered as unimportant [Schultze, 1966; Wallevik, 1979].

The observation of altered plasma decay curves after a bolus injection of tissue enzymes when also zymosan was administeed, an inhibitor of the mononuclear phagocyte system (MPS) (formerly reticulo endothelial system (RES)), led to studies of the action of MPS in protein elimination lMahey, 1965; Mahey, 1967; Wakim, 1963b; Roberts, 1975]. At first sight there emerged the concept that the MPS indeed is involved in the degradation of rapidly disappearing tissue enzymes as $L_{5}, A S T$ and $C K$, in contrast to the situation with slowly eliminated enzymes as $L_{1}$ and ALT. However the enzyme preparations used in these studies often seem to contain a damaged fraction, cf. Section 2.5, which is eliminated very rapidly by the MPS [Freeman, 1958; Hebert, 1976]. Furthermore there are indications that MPS-affecting agents cause enzyme release [Amelung, 1968], also suggested by the data presented in [Wakim, 1963b].

The damaging action of proteases, either directly in the bloodstream or after the transfer of the protein to the small intestine, was also considered as a possible mechanism of removal [Fleisher, 1968; Posen, 1970; Schultze, 1966], with inconclusive results. A gradual degradation of proteins, while circulating in plasma seems improbable from experiments with radiolabelied enzymes showing a simultaneous disappearance of enzymatic activity and radioactivity [Roberts, 1975; Massarat, 1968]. Also the constant value of the Michaelis constant $\mathrm{k}_{\mathrm{m}}$ of the isoenzymes of AST observed during the disappearance from plasma after injection in the dog indicates an integral removal of the molecules Wakim, 1963a]. Other authors however have reported a faster disppearance from plasma of enzymatic activity compared to cadioactivity for $\mathrm{LD}_{5}$ [Querski, 1976] or to plasma levels assayed by immunological methods for CK-MB [Morin, 1979].

The notion emerging from the preceding discussion that degradation of circulating proteins is not well understood, is proba- 
bly best illustrated by the discussion on the question whether the site of removal is situated in an extravascular compartment or in a compartment in close connection to plasma which discussion is entirely based on kinetic studies [McFarlane, 1970] c.f. section 2.4.

\section{Distribution_of_proteins_over_the_body_fliuidis}

The escape of protein from the plasma to the interstitial fluid is by far the most extensively studied aspect of the circulation of proteins through the body. It is now generally accepted that filtration, diffusion and vesicular transport contribute to the passage of macromolecules through the capillary wall. The quantitative description of the transcapillary protein flow commonly proceeds by partitioning this flow in the part effected by diffusion and vesicles $\left(J_{d}\right)$ and the part resulting from the bulk fluid flow over the endothelium. It is assumed that $\mathrm{J}_{\mathrm{d}}$ is determined by the concentration difference between capillary and interstitium:

$$
J_{a}=\operatorname{PS}\left(C_{p}-C_{e}\right)
$$

with

$\mathrm{J}_{\mathrm{d}}$ - the flow of protein $(\mathrm{mol} / \mathrm{h})$ over the membrane

PS - the permeability-surface $(1 / \mathrm{h})$

$\mathrm{C}_{\mathrm{p}}$ - the plasma concentration of the protein (mol/1)

$\mathrm{C}_{e}$ - the extravascular concentration of protein $(\mathrm{mol} / \mathrm{l})$

In Table 2.1 data on capillary permeability derived from [Renkin, 1977] and the review [Renkin, 1979] are summarized. These data show a strong dependence of PS on the size of the molecules. The much larger values of PS found for heart muscle compared to skeletal muscle are related to the higher capillary density in heart.

The fluid flow through the capillary wall is the result of the counteracting forces of the hydrostatic pressure difference 
Table 2.1 Caplilary permeability in heart and skeletal muscle

\begin{tabular}{|c|c|c|c|c|c|c|}
\hline Substance & $\begin{array}{l}\text { Molec. } \\
\text { weight }\end{array}$ & $\begin{array}{l}\text { stokes } \\
\text { radius } \\
(\mathrm{nm})\end{array}$ & $\begin{array}{l}\text { PS } \\
\text { Hear } \\
\text { muso }\end{array}$ & cte & $\begin{array}{l}\text { PS } \\
\text { ske } \\
\text { mus }\end{array}$ & $\begin{array}{l}\text { Letal } \\
\text { de }\end{array}$ \\
\hline urea & 60 & 0.26 & $\begin{array}{r}55000 \\
6600\end{array}$ & $\begin{array}{l}(\mathrm{dog}) \\
(\mathrm{cat})\end{array}$ & 7200 & (man) \\
\hline hexose & 180 & 0.36 & 19000 & (dog) & $\begin{array}{l}3300 \\
2800\end{array}$ & $\begin{array}{l}\text { (man) } \\
\text { (cat) }\end{array}$ \\
\hline suctose & 342 & 0.47 & 14000 & (dog) & $\begin{array}{l}2200 \\
1500\end{array}$ & $\begin{array}{l}\text { (man) } \\
\text { (cat) }\end{array}$ \\
\hline inuline & 550 & 1.5 & 4700 & $(\mathrm{dog})$ & $\begin{array}{l}216 \\
350\end{array}$ & $\begin{array}{l}(\text { man) } \\
\text { (cat) }\end{array}$ \\
\hline myoglobin & 17000 & 1.9 & & & $\begin{array}{l}216 \\
350\end{array}$ & $\begin{array}{l}\text { (man) } \\
\text { (cat) }\end{array}$ \\
\hline albumin & 65000 & 3.6 & 58 & $(\mathrm{dog})$ & $\begin{array}{l}12 \\
14\end{array}$ & $\begin{array}{l}\text { (aog) } \\
\text { (cat) }\end{array}$ \\
\hline$I g G$ & 160000 & 5.6 & 30 & $(\mathrm{dog})$ & 8 & $(\mathrm{dog})$ \\
\hline alpha $_{2}-\mathrm{M}$ & 820000 & 10 & & & 4 & $(\operatorname{dog})$ \\
\hline
\end{tabular}

The values of the pexmeability surface are expressed in $\mathrm{ml} / \mathrm{kg} / \mathrm{h}$. The capillary surface in heart muscle is $56 \mathrm{~m}^{2} / \mathrm{kg}$ and in skeletal muscle $7 \mathrm{~m}^{2} / \mathrm{kg}$.

between the capillary and the interstitium and of the difference in colloid osmotic pressure in plasma and interstitial fluld. The osmotic pressure difference arises from the much smaller permeability of the capillary wall for protelns than for water. Capillary walls are not uniform but show manifold openings and gaps between endothelial cells. The existence of such pores of different sizes explains the decreasing permeability of the wall for molecules of increasing size. Correspondingly the filtration of protein through the membrane associated with the volume flow Jv is determined by the reflection coefficient $\sigma$ of the protein [Kedem, 1963]:

$$
J_{f}=(1-\infty) J_{V} C
$$


with $J_{f}$ - the flow of protein due to filtration (mol/h) over the membrane

$J_{v}$ - the fluid (solvent) flow over the membrane $(1 / \mathrm{h})$

$\sigma$ - the reflection coefficient; represents the fraction of protein (ar other solute) that is excluded from passage when a unit volume of the solution flows through the membrane

$c$ - the concentration of the protein in the pore flow (mol/l); this concentration has a value between $C_{p}$ and $\mathrm{C}_{e}$, due to the diffusional exchange and the paracapillary fluid flow.

For proteins the reflection coefficient has values close to one $(\sigma=0.95-1.00)$ for most capillary walls [Renkin, 1979】, while in heart muscle for urea $a=0.1$ and for sucrose $a=0.3$ is found. Using equations (2.1) and $(2.2)$ one may derive an expression for the lymph concentration. Assuming equality of lymph flow and bulk fluid flow, the amount of protein entering the interstitium is given by $J_{d}+J_{f}$, while in equilibrium the same amount should leave interstitium via lymph:

$$
J_{\ell} C_{\ell}=\operatorname{PS}\left(C_{p}-C_{e}\right)+(1-\sigma) J_{\ell} C
$$

with $C_{l}$ - the protein concentration in lymphe (mol/1)

$J_{\ell}$ - the lymph flow $(1 / \mathrm{h})$.

If further it is assumed that the extravascular concentration $C_{e}$ equals $C_{l}$ and that the concentration $C$ in the bulkflow is the mean of plasma and lymph concentration then elimination of $\mathrm{C}$ and $c_{e}$ from the previous expression results in

$$
\left(\mathrm{PS}+\mathrm{J}_{\ell}-(1-\sigma) \mathrm{J}_{\ell} / 2\right) \mathrm{C}_{\ell}=\left(\mathrm{PS}+(1-\sigma) \mathrm{J}_{\ell} / 2\right) \mathrm{C}_{\mathrm{p}}
$$

i.e.

$$
\mathrm{R}=c_{\ell} / C_{p}=\frac{P S+(1-\sigma) J_{\ell} / 2}{P S+(1+\sigma) J_{l} / 2}
$$


The expcession allows an experimental determination of a and $\mathrm{PS}$ under the assumptions indicated above. Equation $(2.3)$ may be derived more rigorousily [Kedem, 1963] by using the thermodynamics of transport processes and is commonly used in contemporary work on transcapillary transport, although variant forms also appear in the literature as well as different interpretations, e.g. [Rippe, 1979; Renkin, 1979; Taylor, 1981]. Fig. 2.1 shows data on the lymph-plasma concentration ratio $R$ reported in [Grotte, 1956, Renkin, 1979; Hansson, 1975; Olszewski, 19781.

The decreasing permeability for increasing molecular size is apparent from these data as well as from the data in Table 2.1 . This dependence of permeability on molecular weight is mainly found for molecules with a molecular weight smaller than approx-

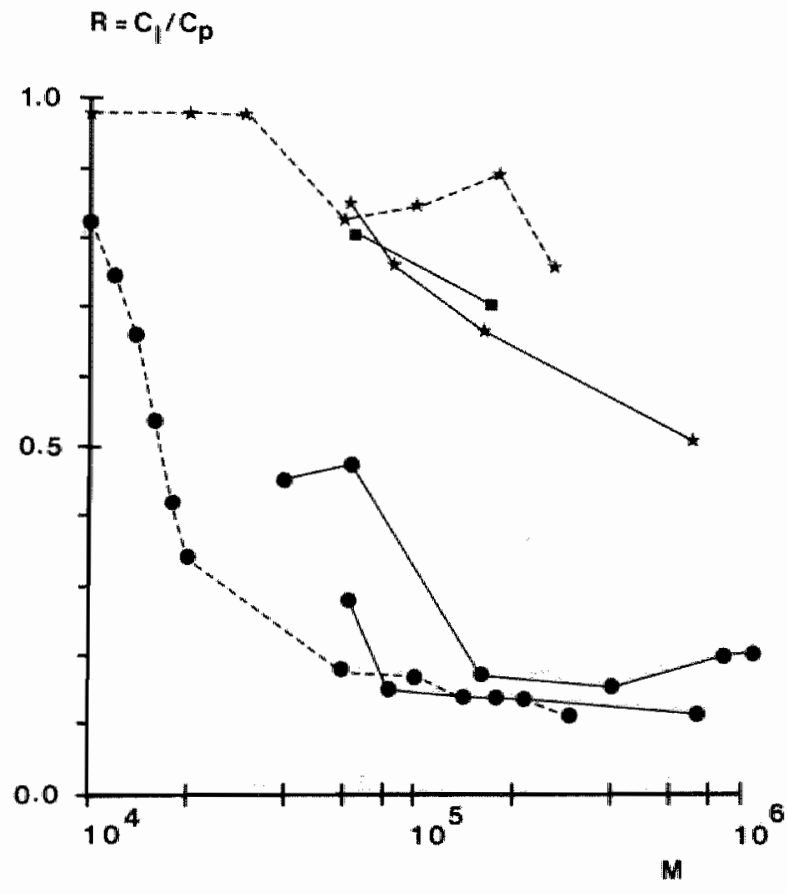

Fig. 2.1 Concentration ratio of macromolecules in 2 mph and in plasma. $M=$ relative moleaular mass. Dotted lines indicate synthetic polymers. Solid lines plasma proteins. $\mathbf{0}=$ leg, $+=$ liver, $\mathbf{0}=$ heart. 
Imately $M=60.000$. For larger molecules it seems that the capillary wall does not longer discriminate with respect to molecular size. This finding is interpreted as a strong indication for the existence of a limited number of large pores. Once the molecular radius exceeds the size of the small pores and this pathway is blocked, the remaining large pores are equally accessible to proteln molecules irrespective of the molecular radius. Fig. 2.1 also shows considerable differences in the data of different authors, which may be due to various high rates of lymphe flow during the experiments, ef. Formula 2.3.

A further discrepancy arises when it is attempted to calculate the extravasation of albumin in the skeletal muscle of an intact animal on basis of the reported PS value. After an intraveneous bolus injection of radiolabeled albumin, the initial disappearance rate from plasma is approximately $5 \% / \mathrm{h}$ in dog and man $(\mathrm{cf}$. Section 2.4). Assuming a muscular mass of $40 \%$ of body weight and PS values of $14 \mathrm{~m} 1 / \mathrm{h} / \mathrm{kg}$ the equivalent of $5.6 \mathrm{ml} \mathrm{plasma} / \mathrm{h} / \mathrm{kg}$ body weight leaves the circulation in the skeletal muscle alone. With a plasma volume of $45 \mathrm{ml} / \mathrm{kg}$ body weight this amounts to an initial disappearance rate of albumin of $13 \% / \mathrm{h}$. This represents a Lower limit because the contribution of filtration was neglected in this calculation.

The transport from interstitium back into the blood circulation is effected by backdiffusion through the capillary vessel wall and by the lymph flow. The relative contribution of both pathways has been the subject of much discussion [Lassen, 1974; Szabo, 1976】. There exists a huge variation in published values of lymph flow and lymph concentrations, reviewed in [Hermens, 19821, which makes quantitative estimates hazardous. Furthermore the major part of thoracic duct lymph is derived from the liver and intestine [Morris, 1956$]$ and thus represents chiefly the short circuit of these organs with relatively permeable capillary walls, while most of the extravascular pool of protein is localised in the skin and the muscles, see below.

The amount of albumin present in the extravascular fluids, 1.e. the size of the extravascular pool is directly examined in a few studies by immunological methods. One stuay reported an 
Table 2.2 Calculation of extravascular pool size (EP) from lymoh

cancentration and interstitial fluid volume (xry)

Skeletal Skin viscera muscle

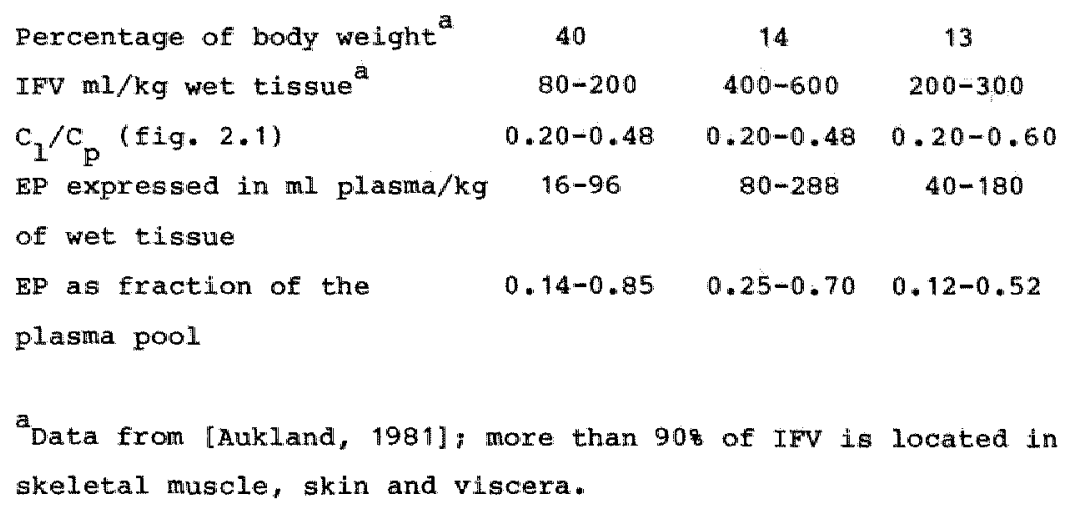

extravascular pool size of 1.6 times the plasma pool [Coward, 1977] which is in agreement with the pool size found in kinetic studies, cf. Section 2.4. Others found however an ratio of 2.5 3.0 [Katz, 1970a; Katz, 1970b; Jewel, 1975].

An alternative approach to estimate the extravascular pool size from the product of the extravascular distribution volume and the extravascular concentration is presented in Table 2.2. In these calculations the exclusion of macromolecules from a part of the IFV, possibly upto 488 [Aukland, 1981] is neglected.

A further assumption is that the lymphe concentration is equal to the concentration in the interstitial fluid. From the data in Table 2.2 it is apparent that especially the uncertainty as to the value of the lymph concentration results in a wide range for the estimates of EP of 0.49 to 2.1 times the plasma pool. 


\subsection{Kinetic studies: Models}

As discussed in the previous section the current knowledge on the process of exchange of macromolecules between plasma and the extravascular fluids is insufficient to derive quantitative estimates of the flows and pool sizes involved, while the process of elimination of native protein from the circulation still is completely obscure. Due to these circumstances nearly all quantitative data on the behavior of proteins in the circulation are derived from kinetic studies, in which the plasma levels (and often the retained dose) in response to an infusion of protein are observed as function of the time. The interpretation of such data requires a model of the dynamics of the protein in the body fluids.

In agreement with the concepts discussed in section 2 all models appearing in the literature share the basic configuration sketched in Fig. 2.2. The total pool of circulating protein is conceived to reside partly in plasma, the plasma pool $P$, and partly in the other (extracellular) body fluids, the extravascular pool E. From the plasma pool $P$ protein disappears at a flow rate $J_{\text {cat }}$ by elimination and at a flow rate $J_{E P}$ by transfer to the extravascular pool. The extravascular pool $E$ increases at a rate $J_{E P}$, representing the extravasation while the pool E diminishes at a rate $J_{P E}$ by the return flow of extravascular protein to plasma. As assumed in some models also extravascular catabolisme at a rate $J_{c a t}^{e}$ occurs. New protein is delivered to the circulation by input in plasma at a rate $f(t)$. Within this framework the differences between models arise from various specifications of the flow rates and more specifically by various configurations assumed for the extravascular pool [Matthews, 1957; Reeve, 1962; Lewallen, 1959a; Beeken, 19621.

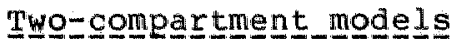

In the simplest conceivable elaboration of the herefore introduced model the flows are assumed to be proportional to the instantaneous size of the pool ( $P$ or $E$ ) providing the flow. This 


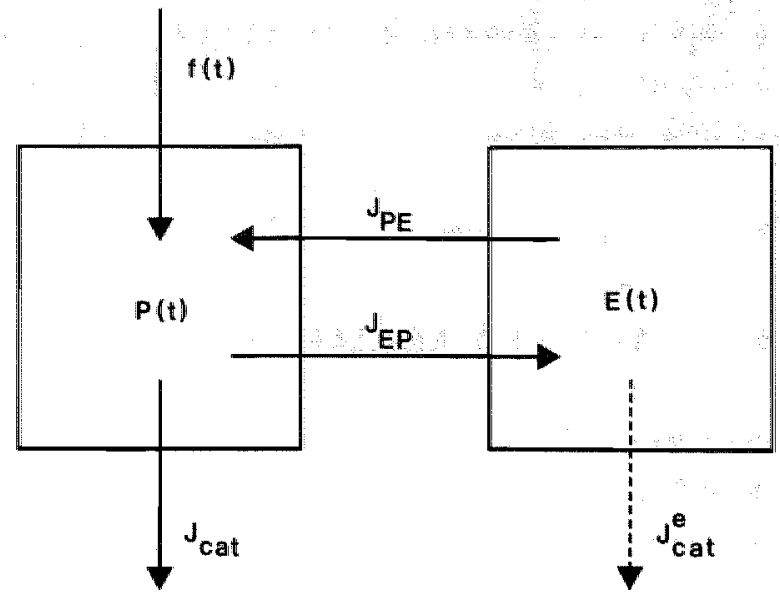

Fig. 2.2 General model for the cinculation of protein in the body's fluido.

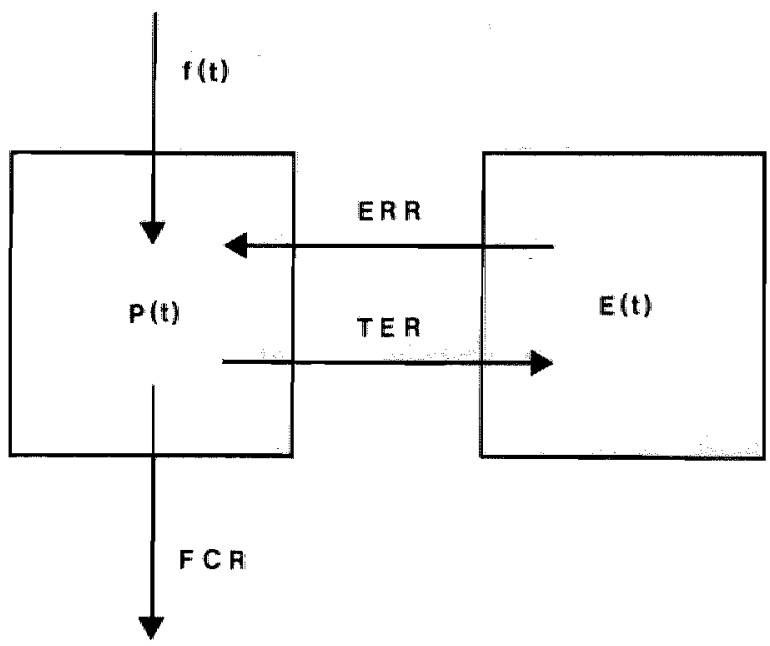

Fig. 2.3 The aimplified two-compartment model. 
leads to the two-compartment model presented in Fig. 2.3 , with the formalism adopted in [Hermens, 1982]. The changes in time of the two state variables, the pool sizes $\mathbb{P}$ and $E$, are given by the system of linear differential equations:

$$
\begin{aligned}
& \frac{d}{d t} P(t)=-F C R P(t)-T E R P(t)+E R R E(t)+E(t) \\
& \frac{d}{d t} E(t)=\operatorname{TER} P(t)-E R R E(t)
\end{aligned}
$$

with $P$ - the quantity of the protein present in plasma, expressed in units (U), e.g. grams for plasma proteins

E - the quantity of the protein present in the extravascular fluids (U)

FCR - the fractional catabolic rate constant $\left(\mathrm{h}^{-1}\right) ; i . e$.

$$
J_{\mathrm{cat}}=\mathrm{ECR} \mathrm{P}
$$

TER - the fractional transcapillary escape rate constant $\left(h^{-1}\right) ;$ i. e.

$$
J_{E P}=\text { TER } P
$$

ERR - the fractional extravascular return rate constant $\left(h^{-1}\right) ; i . e$.

$$
J_{P E}=E R R E
$$

$f(t)$ - the input of new protein into the plasma $(U / h)$.

Using standard methods for the solution of linear differential equations the solution of $(2.4)$ for the initial conditions $P(t=0)$ $=0$ and $E(t=0)=0$ and for $f(t)$ equal to the unit impulse at $t=$ 0 , i.e. the injection of a bolus of one unit in the plasma pool at $t=0$, is given by (cf. Appendix A.2 or [Mathews, 1957]):

$$
\begin{aligned}
& P_{b}(t)=P_{1} e^{-k_{1} t}+P_{2} e^{-k_{2} t}, P_{1}+P_{2}=1 \\
& E_{b}(t)=E_{1} e^{-k_{1} t}+E_{2} e^{-k_{2} t}, E_{1}+E_{2}=0
\end{aligned}
$$

with

$$
\begin{aligned}
& \mathrm{k}_{1,2}=\left(\mathrm{ECR}+\mathrm{TER}+\mathrm{ERR} \pm \sqrt{(\mathrm{FCR}+\mathrm{TER}+\mathrm{ERR})^{2}-4 \mathrm{ERR} F \mathrm{FR}}\right) \\
& \mathrm{P}_{1}=\frac{\mathrm{k}_{1}-\mathrm{ERR}}{\mathrm{k}_{1}-\mathrm{k}_{2}} ; \mathrm{P}_{2}=1-\mathrm{P}_{1} \\
& \mathrm{E}_{1}=-\frac{T \mathrm{ER}}{\mathrm{k}_{1}-\mathrm{k}_{2}} ; \mathrm{E}_{2}=-\mathrm{E}_{1}
\end{aligned}
$$


Thus the system response upon an unit bolus input into plasma $1 \mathrm{~s}$ a biexponential decay curve and the system response upon general input is the so called convolution of the input ith the response to the bolus, cf. Formula 2.12 .

The identification of the three model parameters FCR, TER and ERR specifying this circulatory model is usually performed by the observation of a decay curve $P_{b}(t)$ after an injection of a bolus. In such experiments one generally observes the plasma concentration $C_{b}(t),(U / 1)$, instead of the plasma pool size $P_{b}(t)$, (U). The dilution volume in plasma $V_{p}$ may be determined by extrapolation of $c_{b}(t)$ to zero time:

$$
v_{p}=D / C_{b}(0) \text {, with } D \text { the dose injected }
$$

and $P_{b}(t)$ may then be calculated as $P_{b}(t)=v_{p} C_{b}(t)$. Alternatively ane may express the unit impulse response $\mathrm{P}_{\mathrm{b}}(\mathrm{t})$ as

$$
P_{b}(t)=C_{b}(t) / C_{b}(0)
$$

If the response upon a bolus injection, $P_{b}(t)$, has been measured the model parameters FCR, TER and ERR may be expressed in $P_{1}, P_{2}$, $k_{1}$ and $k_{2}$ by inversion of $(2,5 b)$, cf. Appendix A.2:

$$
\begin{aligned}
& \mathrm{FCR}=1 /\left(\mathrm{P}_{1} / \mathrm{k}_{1}+\mathrm{P}_{2} / \mathrm{k}_{2}\right) \\
& \mathrm{TER}=\mathrm{P}_{1} \mathrm{P}_{2}\left(\mathrm{k}_{2}-\mathrm{k}_{1}\right)^{2} /\left(\mathrm{P}_{1} \mathrm{k}_{2}+\mathrm{P}_{2} \mathrm{k}_{1}\right) \\
& \mathrm{ERR}=\mathrm{P}_{1} \mathrm{k}_{2}+\mathrm{P}_{2} \mathrm{k}_{1}
\end{aligned}
$$

There are several assumptions implicit in this model. Firstly it is assumed that the extravascular pool of the protein is homogeneous with respect to exchange with the plasma pool. Further assumptions concern the linearity and the time-invariance of the system response. These assumptions are cruclal not only for this specific two-compartment model but for practical all methods currently in use for the analysis of the behavior of macromole- 
cules in the circulation. The linearity of the system is plausible when tracer dynamics are considered for instance in the study of radio-labelled (plasma) proteins, cf. Section 2.4. It must, however, be realized that this linearity of tracer dynamics does not imply the linearity of the system with respect to the native protein. For the applications considered in this study, i.e. the description of the dynamics of tissue enzymes in the circulation, some evidence exists for the linearity of the process of elimination of enzyme from the circulation, cf. Section 2.5. As for the linearity of the exchange rates between plasma and the extravascular pool direct evidence is scarce. It might be argued that the processes governing the exchange between these pools, diffusion and filtration, generally show transport rates linearly dependent on the concentrations. Moreover the amounts of tissue enzymes are minute compared to the circulating quantities of plasma proteins. Another assumption made in the above discussed two-compartment model is the absence of extravascular elimination of the protein.

Relaxation of this assumption has serious consequences for the identification of the circulatory parameters. If elimination from the extravascular pool does occur, cf. Fig. 2.4, the equations

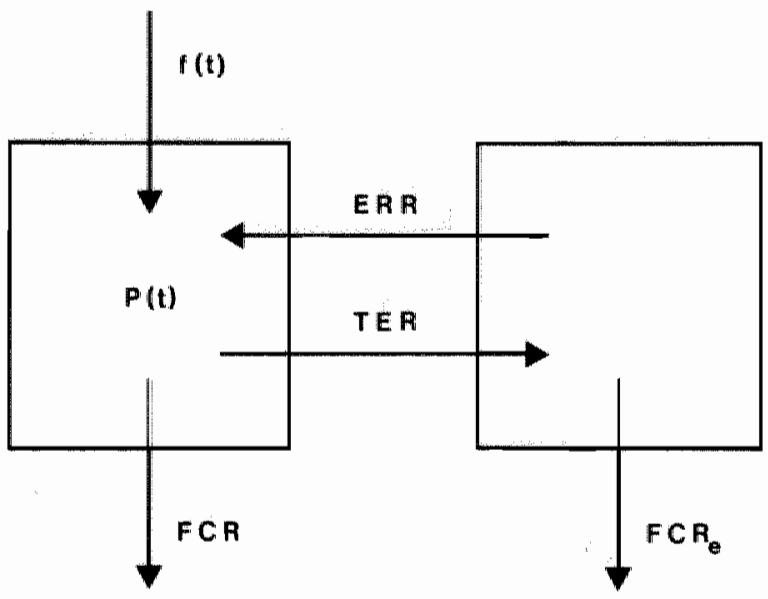

Fig. 2.4 The two-compartment model with extravaseular elimination of protein. 
(2.4) describing the changes in time of $\mathrm{P}(\mathrm{t})$ and $\mathrm{E}(\mathrm{t})$ are replaced by:

$$
\begin{aligned}
& \frac{d}{d t} P(t)=-\operatorname{ECR} P(t)-\operatorname{TER} P(t)+\operatorname{ERR} E(t)+f(t) \\
& \frac{d}{d t} E(t)=\operatorname{TER} P(t)-\operatorname{ERRE}(t)-\operatorname{FCR}_{e} E(t)
\end{aligned}
$$

Using the linear transformation $\mathbb{P}^{\prime}=P, E^{\prime}=\alpha \mathrm{E}$ this system can be rewcitten as:

$$
\begin{aligned}
& \frac{d}{d t} P^{\prime}(t)=-(E C R+T E R) P^{\prime}(t)+(E R R / \alpha) E^{\prime}(t)+f(t) \\
& \frac{d}{d t} E^{\prime}(t)=(\alpha \text { TER }) P^{\prime \prime}(t)-\left(E C R e^{+E R R}\right) E^{\prime}(t)
\end{aligned}
$$

This last system is for the value $\alpha=E R /(E R R+F C R$ ) identical to (2.4) but with different values for the model parameters, indicated by the primed parameters:

$$
\begin{aligned}
& \mathrm{TER}^{\prime \prime}=\frac{\mathrm{ERR}}{\mathrm{ERR}+\mathrm{FCR}} \mathrm{TER} \\
& \mathrm{FCR}^{\prime \prime}=\mathrm{FCR}+\frac{\mathrm{TER}}{\mathrm{ERR}+\mathrm{FCR}} \mathrm{FCR}_{\mathrm{e}} \\
& \mathrm{ERR}^{\prime \prime}=\mathrm{ERR}+\mathrm{FCR}_{\mathrm{e}}
\end{aligned}
$$

The primed parameters in $(2.10)$ are obtained from bi-exponential analysis of the plasma curve of a bolus when it is falsely assumed that elimination occurs only from the plasma pool, cf the model (2.4). Thus an one-parameter family of solutions of (2.8) is found parametrized by the value of $F_{C R}$, which is unidentifiable from the plasma curve alone. However, the requirement that the model is physically interpretable may imply a rather narrow range for the values of the original TER and ERR, cf. Appendix A.2. In such case still meaningful results are obtainable from the analysis of the plasma curve, in spite of the undeterminacy. Observation of $E_{b}(t)$ in addition to $P_{b}(t)$ eliminates this unidentifiability of the circulatory parameters cf. Appendix A.2. 


\section{Three-compartment model 1}

The finding that for some proteins the plasma curves measured after a bolus injection are not adequately represented by a biphasic curve but require a tri-exponential curve for an adequate fit has incited the introduction of three-compartment models in this field. The mammilary model presented in Fig. 2.5, cf. [Matthews, 1957], is the most frequently employed for the analysis of plasma disappearance curves. In this model it is assumed that elimination takes place from the plasma pool and that the plasma pool exchanges protein with the independent extravascular pools. The unit impulse response for this model is given by:

$$
P_{b}(t)=P_{1} e^{-k_{1} t}+P_{2} e^{-k_{2} t}+P_{3} e^{-k_{3} t}
$$

Expressions for $P_{1}, P_{2}, P_{3}, k_{1}, k_{2}$ and $k_{3}$ as a function of the model parameters FCR, $\mathrm{TER}_{1}, \mathrm{TER}_{2}, \mathrm{ERR}_{1}$ and $\mathrm{ERR}_{2}$ are given in Appendix A.3. The same is true for the reverse problem i.e. the calculation of the model parameters from the coefficients of the tri-exponential unit impulse response.

The five rate constants of the mammilary model are identifiable from the five parameters characterizing the unit impulse response; however, it was hereby assumed that the extravascular pools do not exchange protein and that elimination occurs only in plasma. Thus the mammilary model is a special case from the general three-compartmental model presented in Fig. 2.6. Although there exists indeed some evidence for the existence of different rate of exchange of protein between plasma and the interstitial fluids of different tissues, e.g. skin and muscle, [Katz, 1970a; Donato, 1966] giving a motivation for the three-compartmental model, there are few reasons to suppose a priori that proteins are not eliminated from the extravascular pools or that there is no exchange between these pools.

The relaxation of these assumptions makes the general threecompartmental model unidentifiable from the observation of the plasma disappearance curve after a bolus injection alone. While 


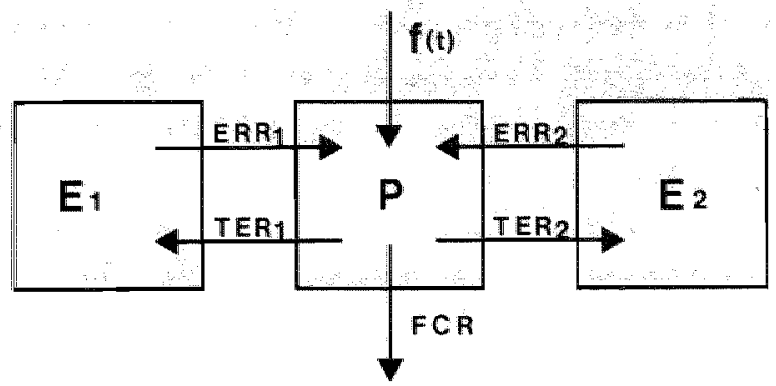

Fig. 2.5 Mathews mammilary three-compartment modet.

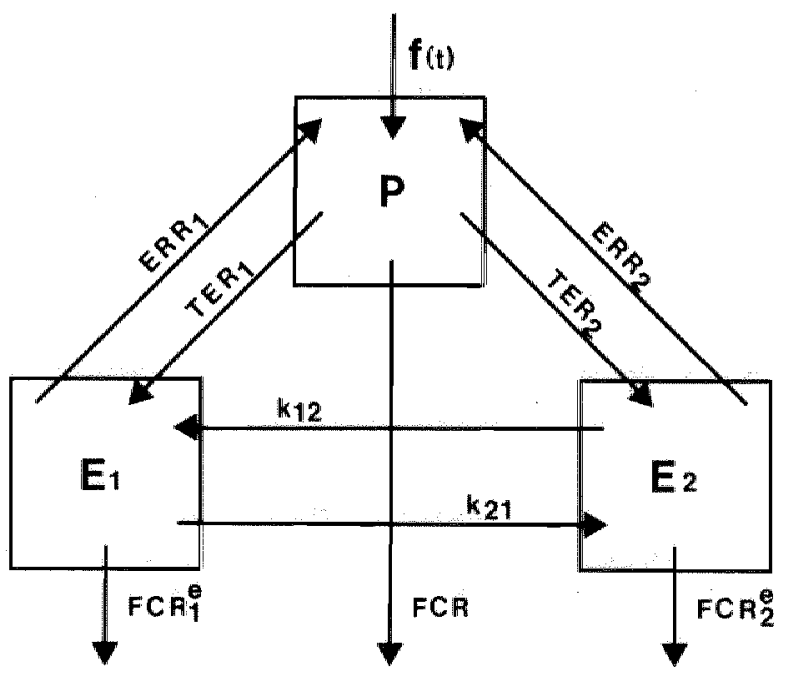

Fig. 2.6 The general three-compartment model. 
in the case of the two-compartment model the observation of the total extravascular pool size as a function of the time restored the identifiability this is not the case for the three-compartmental model. Just as is the case in the previous example of the two-compartment model it is possible to generate an family of models compatible to the data [Berman, 1956; Lewallen, 1959a,b; Walter, 1981]. In case that the plasma pool $P$ and the total extravascular pool $E_{1}(t)+E_{2}(t)$ are observed the linear transEormation

$$
P^{\prime}=P ; E_{1}^{\prime}=\alpha E_{1}+\beta E_{2} ; E_{2}^{\prime}=(1-\alpha) E_{1}+(1-\beta) E_{2}
$$

still is compatible with the data and a two-parameter family of models can be generated from the data. It appears, however, that also in this case for a slowly eliminated protein as albumin the restriction to physically interpretable models results in a rather narrow range for the possible parameter values [Lewallen, $1959 \mathrm{a}, \mathrm{b}]$.

The unidentifiability of the general three-compartmental model only can be resolved by more observations, e.g. direct measurement of the elimination in the extravascular pools or observation of the separate extravascular pools, or by study of the system response to protein input in the extravascular pools. For a convenient identifiability analysis see e.g. [Delforge, 1981; Norton, 19801. Such extensions of the experimental procedures are even more necessary for a meaningfull application of more complex compartmental models [Jacquez, 1972; Brown, 1980]. This would require that a more precise physiological meaning could be attributed to the various compartments and the transfer processes between them.

With these data lacking all models used are essentially "black box" models as far as the site of elimination and the structure of the extravascular protein pool is concerned. Most results hinge on an exponental analysis of the plasma curves and the estimation of the parameters $P_{i}$ and $k_{i}$ in

$$
P_{b}(t)=\sum_{i=1}^{m} P_{i} e^{-k_{i} t} ; P_{1}+\ldots+P_{m}=1
$$


is a notoriously 111 conditioned problem, extcemely sensitive even for independent random errors in observed values of $P_{b}(t)$ and even more sensitive for minor systematic contamination of the data.

This is illustrated in Table 3.2 , where an error free triexponental plasma curve according to the mamilary model is simulated, and is shown to be approximated closely by a bi-exponential curve. The two-compartment model extravascular pool size so calculated however is a serious underestimation of the total extravascular pool size of the mamilary model.

Model-independent_methods_of__analysis

Once the assumption can be made that the system response is (linear) additive and time invariant, much information can be derived from the observation of the response curves of the plasma levels $P_{b}(t)$ and the total extravascular pool size $E_{b}(t)$ after $a$ injection of a bolus at zero time. Especially the synthesis rate of a protein delivered directly into plasma and the extravascular pool size in the steady state may be derived explicitly from such data.

In the simplified two-compartment model $(2.4)$ the extravascular poolsize in equilibrium (E/P) $\mathrm{s}$ equals:

$$
\frac{d}{d t} E_{s}=0=T E R P_{s}-E R R E_{s}:(E / P)_{s}=T E R / E R R
$$

For the mammilary model an analogous expression

$$
(\mathrm{E} / \mathrm{P})_{\mathrm{S}}=\left(\mathrm{E}_{1} / \mathrm{P}\right)_{\mathrm{s}}+\left(\mathrm{E}_{2} / \mathrm{P}\right)_{\mathrm{s}}=\left(\mathrm{TER}{ }_{1} / \mathrm{ERR}_{1}\right)+\left(\mathrm{TER}_{2} / \mathrm{ERR}_{2}\right)
$$

may be derived. The predicted synthesis rate in both models is obviously:

$$
S=F C R P_{s}
$$

The above expressions depend on an exponential analysis of the plasma curve followed by the interpretation of the exponential 
coefflcients in the framework of a selected compartmental model. One may however start equally well from the assumption that the system response is additive and time invariant [Cutler, 1978; Nossiin, 1964]. In that case the responses $P(t)$ and $E(t)$ upon an input $f(t)$ may be expressed in terms of the unit impulse responses $\mathbb{P}_{b}(t)$ and $E_{b}(t)$ by means of the so called convolution integrals, cf. Appendix A. I:

$$
\begin{aligned}
& P(t)=\int_{0}^{t} f(\tau) P_{b}(t-\tau) d \tau=f * P_{b}(t) \\
& E(t)=\int_{0}^{t} f(\tau) E_{b}(t-\tau) d \tau=f * E_{b}(t)
\end{aligned}
$$

Inserting a constant synthesis rate $f(t)=S$, in both equations we obtain for the steady state values

and

$$
\mathrm{P}_{\mathrm{s}}=\mathrm{S} \int_{0}^{\infty} \mathrm{P}_{\mathrm{b}}(\tau) \mathrm{d} \tau
$$

$$
E_{s}=s \int_{0}^{\infty} E_{b}(\tau) d \tau
$$

Hence it is found that

and

$$
S=P_{S^{\prime}} \int_{0}^{\infty} P_{b}(\tau) d \tau
$$

$$
(E / P)_{s}=\int_{0}^{\infty} E_{b}(\tau) d \tau / \int_{0}^{\infty} P_{b}(\tau) d \tau
$$

If the extra assumption is made, that elimination occurs from plasma only, we even can do without $E_{b}(t)$ because then the amount $D_{b}(t)$ of protein degraded up to time $t$ can be calculated by

$$
\begin{aligned}
& F C R=1 /\left(\int_{0}^{\infty} P_{b}(\tau) d \tau\right) \\
& D_{b}(t)=F C R \int_{0}^{t} P_{b}(\tau) d \tau
\end{aligned}
$$

The quantity of protein in the extravascular pool is then derived from the balance equation, cf. [Nosslin, 1964]:

$$
l=P_{b}(t)+E_{b}(t)+D_{b}(t) .
$$




\subsection{Kinetic studies: plasma proteins}

In healthy individuals plasma protein concentrations have relatively high values and it is not practicable to perform kinetic studies simply by infusion of bulk protein. Administration of a manageable dose only would result in a small deviation from the normal steady state condition. Therefore kinetic studies on the behavior of plasma proteins in the circulation generally use (radio-) labelled proteins. Since the fifties the availability of the iodine isotopes ${ }^{131} \mathrm{I}$ and ${ }^{125} \mathrm{I}$ and the techniques for tagging proteins with these labels provoked a proliferation of reports on this kind of studies. Initially various artefacts were caused by the purification and labeling procedure. In general this kind of damage inflicted to the native protein, e.g. by overlabelling, has the effect that the affected fraction of protein exhibits an anomalously rapid elimination [Schultze, 1966; Rossing, 1967]. In this respect however serious progress has been made by the introduction of various checks: comparison of the disappearance rate of native and cadiolabelled albumin in analbumenics [Ereeman, 1959b], the rat liver test [Cohen, 1961], and biological screening, where the labelled protein preparation is injected in an experimental animal, so that the damaged fraction of protein is eliminated and the remaining fraction is harvested from the plasma.

With these labelling artefacts recognized and controlled the use of radioactively tagged proteins provides many advantages. Assuming an ideal label the transfer rates for the tagged protein satisfy irst order rate laws: If the transfer of the native protein, e.g. from pool 1 to pool 2 is given by $J_{21}$, then the How of the indistinguisable label from pool 1 to pool 2 equals

$$
J_{21}=J_{21} \mathrm{P}_{1} / \mathrm{P}_{1}=\left(J_{21} / \mathrm{P}_{1}\right) \mathrm{P}_{1}
$$

with $\mathrm{P}_{1}$ and $\mathrm{p}_{1}$ respectively the amount of the native and the tagged protein in pool 1. Thus even for non-linear rate equations governing the dynamics of the native protein the tracer kinetics may be described by linear rate equations, because $J_{21} / P_{1}$ is 
hardly influenced by the addition of tracer amounts of labelled proteins and thus remains constant.

Radiolabelled proteins allow high accuracy of determination and I-Labelled proteins have the additional advantage that the iodine Label trom degraded protelns appears after a relatively short cicculation time in the urine [Takeda, 1962; Zizza, 1959]. It is conceived that after degradation of the protein the label, perhaps incorporated in a small organic compound, is released in the distribution space of iodine, which is considecably larger than the distribution volume for proteins and generally considered as one well mixed pool, from which the label is excreted in the urine, cf. Fig. 2.7. Iodine kinetics are then described by [Lewallen, 1959a; Beeken, 1962; Takeda, 1962]:

$$
\begin{aligned}
& \frac{d}{d t} I(t)=-k_{u} I(t)+v \operatorname{DR}(t) \\
& \frac{d}{d t} U(t)=k_{u} I(t)
\end{aligned}
$$

with $D R(t)$ - the amount of protein eliminated per hour

$I(t)$ - the circulating amount of iodine

$\mathrm{U}(t)$ - the (cumulative) amount of label excreted in urine

$v$ - number of iodine labels per protein molecule

with $\mathrm{k}_{\mathrm{u}}=0.072-0.084 \mathrm{~h}^{-1}$. The cumulative amount of degraded protein can then be expressed as

$$
D(t)=\int_{0}^{t} D R(t) d \tau=\left(U(t)+\frac{1}{k_{u}} \frac{d}{d t} U(t)\right) / v
$$

Thus an independent estimation is obtained for the quantity of protein eliminated. Such an estimate allows also the determination of the total amount $\mathbb{E}(t)$ of protein present in the extravascular pool using the relation

$$
1=P(t)+E(t)+D(t)
$$

which holds after the injection of one unit of protein.

This allows the model-independent determination of the syntesis rate and of the size of the extravascular poolsize, as 
Fig. 2.7 Compartment model for the iodide circulation and exoretion.

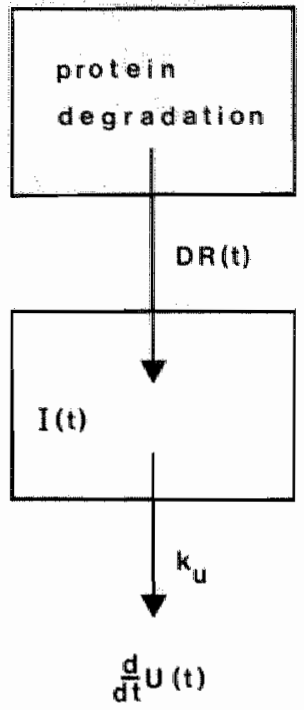

described in the previous section, of. [Andersen, 1964]. Another application of the $D(t)$ so obtained is the independent check on the FCR as obtained by the interpretation of the plasma curve by means of e.g. Mathews mammilary compartmental model. In this way the risk that a rapidly declining initial phase of the plasma curve, caused by the presence of a damaged fraction of labelled protein is falsely interpreted as reflecting a distribution phase, is avoided. Furthermore the independent assessment of the elimination rate by means of (2.14) allows some conclusions on the site of elimination. There is some time required tor protein injected into the plasma to reach the extravascular pool and thus elimination from (one of) the extravascular pools would imply a delayed urinary excretion compared to the situation that elimination takes place from the plasma pool. In this respect the plot of $D(t)$ against $f^{t} P(s)$ ds could be of diagnostic value exhibiting a straight line through the origin with slope FCR in case of elimination from plasma and showing deviations from this line in case of extravascular elimination, see also the integrated rate equation method of [Nosslin, 1973]. The opposite finding of a delayed urinary excretion however although generally considered as an indication of extravascular elimination [Lewallen, 1959a; Mosslin, 1973; Alper, 1963; McFarlane, 19701 could also be caused 
by a time-delay between removal from the circulation and release of the lodine label.

By using the urinary excretion rate in the above described way a number of authors have concluded that albumin and immunoglobin-G elimination occurs in plasma [Takeda, 1963; Beeken, 1963; Andersen, 1964). This conclusion is however challenged by others [Lewallen, 1959a, McFarlane, 1970].

In Table 2.3 a selective compilation of studies on the behavior of plasma proteins in the circulation is presented. Reports are included that give data on the values of FCR, TER and $(E / P)$, calculated by one of the methods discussed in the previous section, or that present sufficient data of the observed plasma disappearance curve so that these parameters could be calculated by using the two-compartment model or the mammilary three compartment model are included. A number of the earlier studies that are suspicious for labeling artefacts were excluded. From the data presented in this table it appears that within one study the circulatory parameters are well defined and exhibit a moderate variance but it is also apparent that there exist a number of disagreements between different studies concerning the same pratein, e.g. for albumin and fibrinogen. For the FCR and $(\mathbb{E} / \mathrm{P}) \mathrm{s}$ generally a variation of less then $20 \%$ is observed while TER exhibits frequently a coefficient of variation of $30 \%$.

In general the plasma curves are reported to conform well to a bi-exponential curve with as notable exceptions albumin and also alpha $_{1}$-antitrypsin.

From the plot in Fig. 2.8 of the parameters TER and (E/P) $\mathrm{s}$ versus the molecular weight there appears no consistent relation. It seems to be only possible to infere from these plots a range for the values of $\operatorname{TER}\left(\mathrm{h}^{-1}\right)$ and $(\mathrm{E} / \mathrm{P}) \mathrm{s}^{*}$

$$
0.01<\mathrm{TER}<0.03
$$

and

$$
0.5<(\mathrm{E} / \mathrm{P})_{\mathrm{S}}<1.4
$$

for molecules with a molecular weight between 80.000 and 150.000 . 
Totole 2.3 Glaculatory parameters af plasmo proteingit

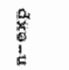

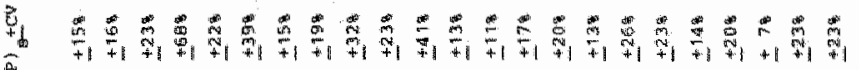

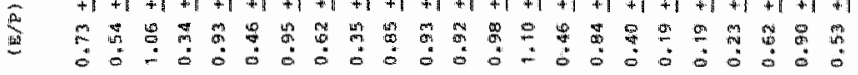

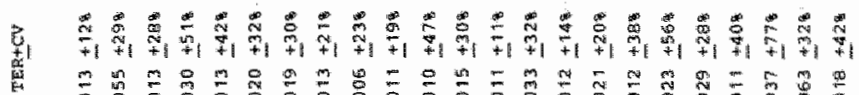

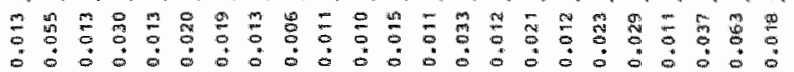

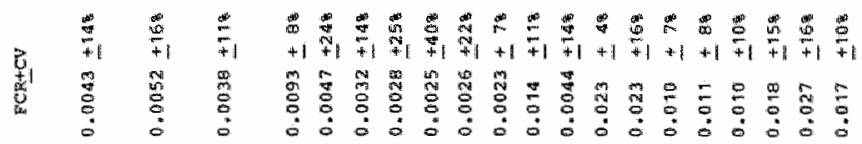

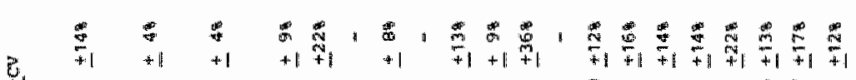

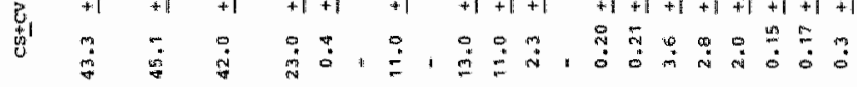

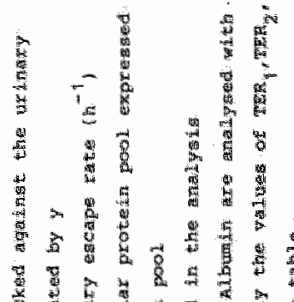

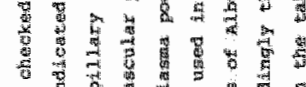

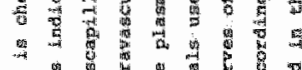

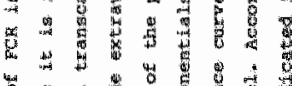

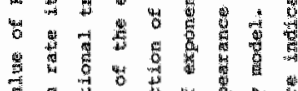
5
5

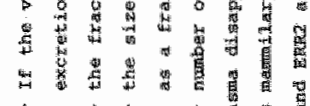

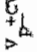

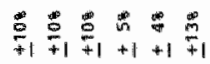

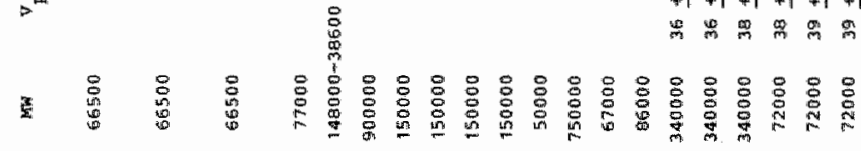

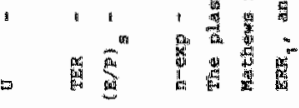

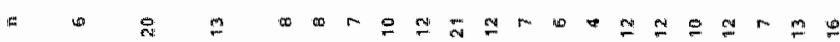

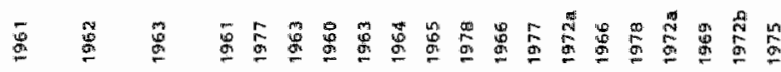

I.

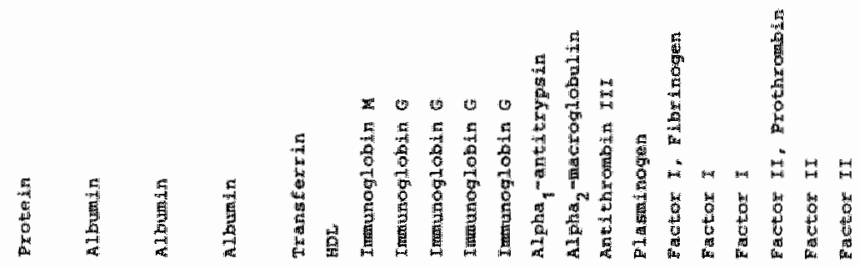

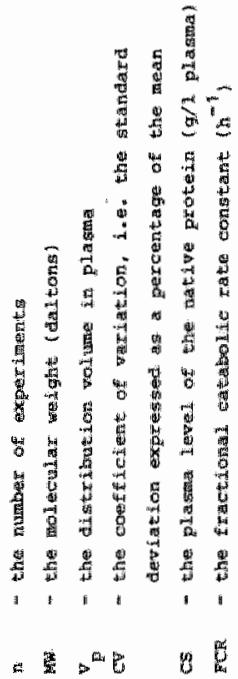




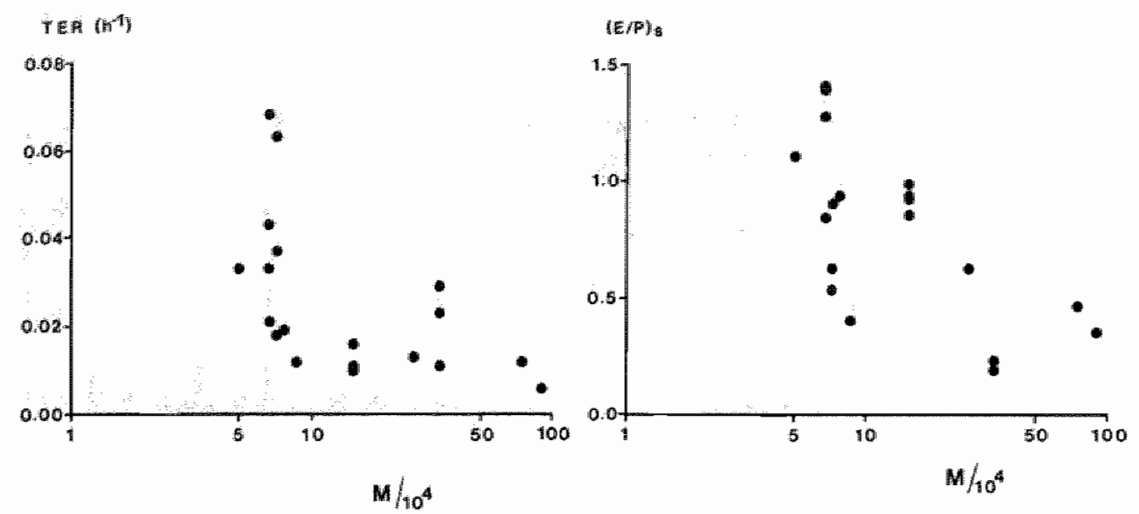

Fig. 2.8 Tranecpaillary escape rate (TER; left panel) and extravascular poot size $(E / P)_{G}$; right panel) in relation to molecular weight $(M)$. Data from Table 2.3. 


\subsection{Kinetic studies: Tissue enzymes}

In contrast to the wealth of information avaliable on the behavior in the circulation of plasma proteins in man the information on the behavior of tissue enzymes in this respect is fragmentary. Only one study exists where the plasma aisapppearance curve of a tissue enzyme is followed after injections of an enzyme preparation in man [Clubb, 1965]. The results obtained in this study on placental alkaline phosphatase fit quite well in the picture that emerged from studies on plasma proteins. For this molecule with $M=190.000$ a TER of $0.028\left(\mathrm{~h}^{-1}\right)$ and $(\mathrm{E} / \mathrm{P})_{\mathrm{s}}=$ 0.70 was found. Two further reports exist on the apparent disappearance rate observed after reinfusion of enzyme rich plasma obtained from patients with high plasma levels of tissue enzymes in the same patients after renormalization of their plasma levels [Dawson, 1969; Tommasini, 1979].

More data are available for the case of $\mathrm{CK}$ in the dog, cf. Table 2.4. This compilation may illustrate the problems caused by purification of enzymes. Frequently a very fast initial elimination phase is observed [She11, 1971; Sobel, 1977], which was attributed to an extraordinary rapid escape to the extravascular pool (TER $=0.60 \mathrm{~h}^{-1}$, [Sobel, 1977]) by these authors. This interpretation led to a value of $\mathrm{FCR}=0.54 \mathrm{~h}^{-1}$. Others attributed this biphasic disappearance of $C K$ to a fraction of damaged molecules present in the preparation IVisser, 19810; Hermens, 1982]. This is corroborated by the circulatory parameters in the dog as obtained with radioactively labelled plasma proteins and ALT, cf. Table 2.5, that conform to the circulatory model as discussed in the previous section for man. With the values of TER and ERR from these data the disappearance curve of a rapidly eliminated enzyme such as $C K$ should be virtually mono-exponential. Thus the interpretation of the rapidly decreasing phase of the plasma curve as caused by an initial distribution phase must be rejected. That enzymes are easily affected by purification is also apparent from observation of anomalous distribution volumes. In a study where purified preparations of $C K-M M, C K-M B$ and $C K-B B$ were injected in the dog a distribution volume of $67 \pm 18 \%, 78 \pm$ 
Table 2.4 Plama as apgearance of CK-wa preparations injected in

the: $\mathrm{dog}$.m.

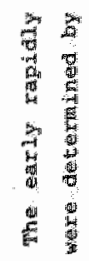

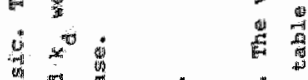

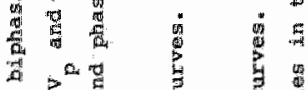

i $p^{2}$

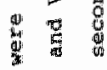

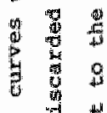

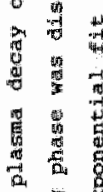

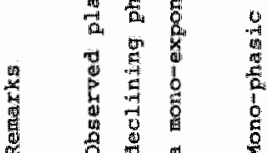

辛

要要

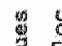

.

(j)

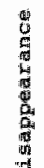

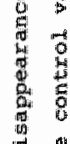

章

曾

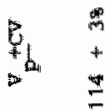

$\therefore \quad=$

s.

造

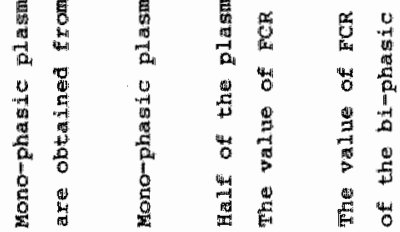

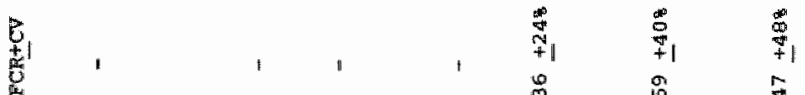

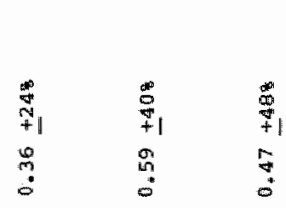

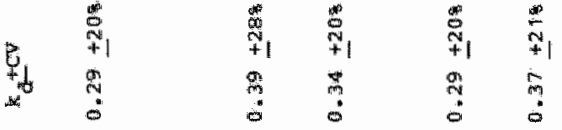

$\stackrel{\infty}{+\infty}$

$\stackrel{\leftrightarrow}{+\infty}$
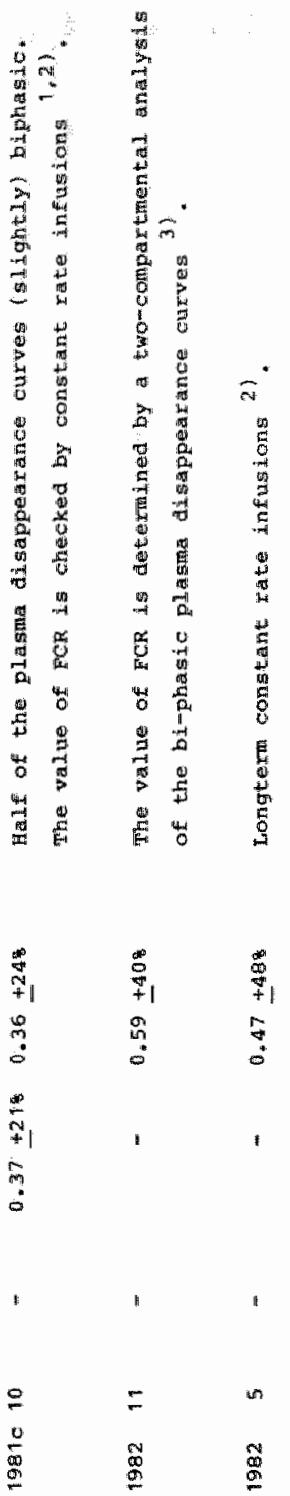

it
- के

尊

울

点

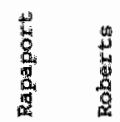

焉

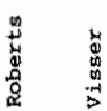

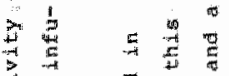

苟

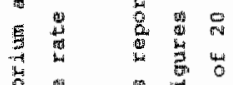

焉

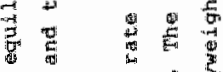

要三容要

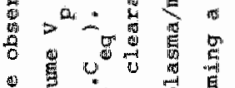

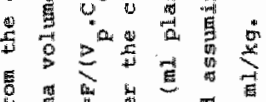

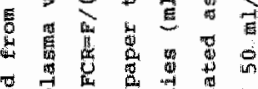

过等

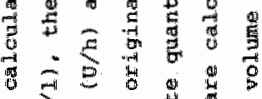

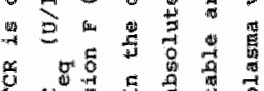

का के

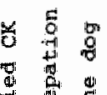

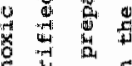

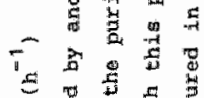

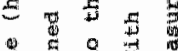

通 8

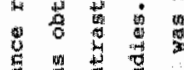

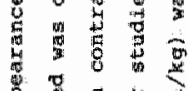

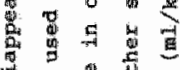

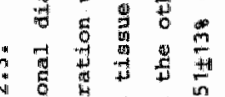

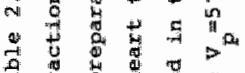

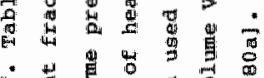

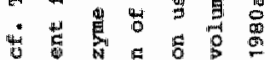

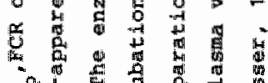

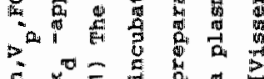


Table 2.5 Circulatory parameters of plesma protedne and enzytes in the dogin

\begin{tabular}{|c|c|c|c|c|c|c|c|c|}
\hline Protediti & Reference & & $\mathrm{n}$ & Whit & $c_{g}+c$ & $\mathrm{FCR}_{-} \mathrm{CO}$ & TERAE & $(\mathrm{k} / \mathrm{p})+\mathrm{cv}$ \\
\hline Albumin & Wetterfors & .1965 & 6 & 65500 & $33.4 \pm 78$ & $0.0074+58$ & $0.034 \pm 230$ & $1.09 \pm 198$ \\
\hline Ima noglobin $G$ & Aradersen &, 1963 & 10 & 150000 & $9.9 \pm 12 \%$ & $0.00105+239$ & $0.010+25 \%$ & $0.72 \pm 313$ \\
\hline Antithrombin III & Kobayrasint. & .1977 & 20 & 67000 & $0.6 \pm 19$ & $0.025 \pm 70$ & 0.048208 & $0.42 \pm 90$ \\
\hline ALT & 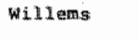 & .1982 & 6 & 115000 & $13.0 \pm 49$ & $0.022 \pm 220$ & $0,0.31 \pm 400$ & $0.48+30 \%$ \\
\hline
\end{tabular}

CE. Table $2.3 \mathrm{cs}$ of hit express in $\mathrm{U} / \mathrm{l}$.

228 and $104 \pm 238(\mathrm{ml} / \mathrm{kg})$ (mean $\pm \mathrm{CV}$ ) where observed for these enzymes [Rapaport, 1975]. In another study using non-purified preparations of CK, LD, AST and ALT virtually the same plasma volumes ranging from 47 to $51(\mathrm{ml} / \mathrm{kg})$, were found for these four enzymes [Visser, 1982]. This sensitivity of tissue enzymes for purification procedures is not restricted to the case of CK. Similar rapidly eliminated fractions are apparently present in purified preparations of $C-A S T$ and ALT explaining the occurrence of triphasic plasma curves of the injection in the dog [Wakim, 1963a; Fleisher, 1963a] in contrast to strictly mono-phasic plasma curves observed after injection of AST [Visser, 1981c] and a biphasic plasma curves observed after injection of the much slower eliminated ALT [Willems, 1982], when using a preparation obtained by anoxic incubation of tissue.

The data in Table 2.4, accepting the equivalence of $k_{d}$ and FCR, indicate a considerable influence of the preparation used on the value of FCR obtained, but it also appears that within the same study the variation in FCR observed is moderate; the coefficient of variation generally does not exceed 20\%. This same order of magnitude is also seen in the coefficients of variation of FCR of plasma proteins, cf. Table 2.3 and Table 2.4, and in the variation of the values of FCR for ALT, GPI and AST IWI1lems, 1982; Visser, $1981 \mathrm{cl}$. As these coefficients of variation also comprise the random error due to the inaccuracy of the method $1 t$ is assumed that the biological variation in FCR of tissue enzymes is in the range of 15-20\%.

Especially for $\mathrm{CK}$ much attention has been given to factors influencing the apparcut disappearance rate from the plasma in 
the dog [Roberts, 1975; Roberts, 1977]. It appears that the injection of zymosan, already mentioned in section 2, morphine in high dosis, valium in high dosis and various kind of anaesthesia reduce the elimination rate of $\mathrm{CK}$, wile hemodynamic disturbances do not exhibit an effect on the elimination rate.

As mentioned in the previous section it is demonstrated for several plasma proteins that the fractional catabolic rate FCR depends on the plasma level of the protein. Such a deviation from a first order rate process is not reported up to now for enzymes. on the contrary there exist several indications that the elimination of enzymes is a first order rate process, i.e. the amount eliminated per hour equals a constant factor (FCR) times the plasma concentration.

The strictly mono-exponential plasma curves observed after injection of AST is incompatible with a nonlinear dependence of the elimination rate on the plasma concentration. A more direct evidence is the apparent independence of the FCR from the injected dose [Visser, 1981C, Rapaport, 1975] and the equality of FCR's as obtained with bolus injections and with constant rate infusions [Visser, 1981c; Willems, 1982]. 


\subsection{Entrance into plasma of tissue enzymes}

In the normal healthy state the plasma levels of tissue enzymes such as CK and LD are low. The intracellular concentration of $\mathrm{CK}$ in skeletal muscle for instance exceeds the plasma concentration by a factor 50.000. The existence of such large gradients evoked the doctrine that tissue enzymes in normal tissues are confined to the cell and that leakage of these enzymes out of the cell implies tissue necrosis. There exist however several indications that such a strict relation between cellular enzyme loss and cell death does not hold in absolute generality. It is well known that after heavy exercise, e.g. long distance running, the plasma levels of $\mathrm{CK}$ may show a considerable rise [Hansen, 1982; Berg, 19821, while also focal cellular damage is observed in exercised muscles in rat [Kuipers, 1983]. This damage however appears to be transient indicating a capacity for regemeration of the skeletal muscle cell. In experiments where erythrocytes were exposed to a osmotic shock a transient leakiness of the cell membrane for macromolecules followed by a resealing of the membrane was observed [Seeman, 1974]. Another outstanding example of cell recovery after the infliction of lesions to the plasma membrane is the regeneration of Nitella cells after integral removal of the plasma membrane [Inoue, 1973].

As for the myocardium the data on heart lymph concentrations also suggest that enzyme release is a normal phenomenon. The concentrations of cardiac enzymes in heart lymph are higher than in plasma, the isoenzyme pattern of LD in cardiac lymphe resembles closely the pattern in heart tissue in contrast to the pattern found in plasma [Norbeck, 1977; Szabo, 1978b; spieckermann, 1979]. Moreover it was demonstrated that isoprenaline induced stress increased the lymphe flow as well as the amount of cardiac enzymes transported by the lymph [Norbeck, 19771. However in these experiments the quantities of enzyme involved are very small compared to the quantity present in the heart, e.g. the amount of $\mathrm{CK}$ transported during one hour is less than 0.1 of the total heart content. Obviously a minor degree of tissue damage inflicted during the preparation phase would be 
sufficient to cause the observed enzyme release and the increased release during exertional stress, accompanied by an increased lymph flow, could then also be explained by an enhanced wash-out.

Notwithstanding the uncertainties brought about by the above mentioned examples it is concluded in a review that for the working heart enhanced enzyme rellease during and after a hypoxic or ischemic episode indicates cell death [Hearse, 1979]. This conclusion is supported by the findings that in experimental coronary occlusions a close correlation between lacal enzyme depletion and resialual local coronary flow is observed [Hirzel, 19771, that in hypoxic perfused isolated hearts a close correlation is observed between enzyme release and the depletion of high energy phosphates [Gebhard, 1977] and that in anoxic heart cell cultures the extent of cell death measured by the cellular uptake of Tryptan Blue was strictly correlated to the quantity of enzyme released [Van der Laarse, 1980a】.

At first impression the initial rising part of the plasma activity curves of the enzymes $C K$ and $H B D$ as observed in patients with AMI suggests a retarded release of HBD compared to $C K$. This erroneous conclusion is caused by the steeper initial increase of CK due to the higher myocardial CK content [Willems, 1979]. On the contrary there exists much evidence that once the cell envelope becomes permeable for macromolecules, all cytosolic enzymes leave the cell simultaneously. This was demonstrated in a preparation of beating neonatal rat heart cells, where anoxia induced release of $C K$, HBD and cAST runs strictly parallel [Van der Laarse, 1978, Van der Laarse, 1981]. In vitro hypoxic incubation of chicken muscle also was shown to induce a parallel release of LD, CK, MD, ALD, PGM and phosphorylase b, proteins with a molecular weight ranging from $M=70.000$ to $M=370.000$ [Dawson, 1966]. It thus appears that the leaky cell membrane does not discriminate between molecules of different sizes. Phis parallel release of enzymes is also observed in experiments with isolated perfused hearts, where anoxia and hypoxia induced release of myokinase $(M=21.000)$ and myoglobin $(M=17.600)$ was not accelerated in comparison to the release of $\mathrm{CK}(\mathrm{M}=80.000)$ or LDH $(M=136.000)$ [Hearse, 1973; Mezger, 1979; Spieckermann, 
1979]. These observations obtained with cell cultures, in vitro incubated tissue and isolated perfused hearts are confirmed in studies on the in vivo release of various emzymes following cardiac damage. After experimental infarctions in the dog the plasma levels of CK, AST, ALT, LDH show a simultaneous rise CNorbeck, 1977: Norbeck, 1978]. In the same preparation ilt was earlier demonstrated that in heart lymph various cardiac enzymes show simultaneous elevation and that the lymphe concentrations of these enzymes are in proportion to myocardial cytoplasmic enzyme content [Malmberg, 1973]. This simultaneous release of enzymes in heart lymph is confirmed in later studies for the case of coronary ligation in the dog [Norbeck, 1977] and in man after cardiac surgery [Inoue, 1978].

The above mentioned examples regard the release of cytoplasmic enzymes. The release of enzymes from other cellular compartments in contrast may be considerably delayed and incomplete. In the above mentioned study of Malmberg it was demonstrated that the release of mitochondrial AST was small compared to the release of cytoplasmic AST. In patients after AMI the release of mitochrondrial AST and MDH is considerably delayed in comparison to the appearance in plasma of cytoplasmic enzymes [Smith, 1977]. Similar observations are reported in [Van der Laarse, 1981] where also the incomplete release of mAST from anoxic cell cultures is demonstrated.

Enzymes leaked out of the cell in the Interstitial fluda arrive in the plasma partly by lymphatic transport and partly by diffusion directly into the capillaries. Although in experimental infarctions the lymphatic enzyme activites may reach huge values the total transport by lymphe is restricted by the low flow rate of cardiac lymphe and it is estimated that only 15-30\% of the enzyme appearing in plasma is transported by lymphe [Malmberg, 1973; Norbeck, 1977; Spieckermann; 1979]. This is in agreement with the high values found for the permeability surface product for heart capillaries, c.f. Table 2.1. A further factor that may facilitate the transport from interstitium into the capillaries in the heart compared to the resting skeletal muscle is the build up of a considerable tissue pressure during the systolic phase of 
every heart beat, cf. Section 2. The above mentioned conclusion of the relative contribution of lymph to the transport from tissue to plasma is obtained by direct estimation of the quantity of enzyme transported by lymph. Other authors on basis of circumstantial evidence have drawn the opposite conclusion IClark, 1978; Gervin, 19741. Accepting however the conclusion that only a small part of the enzymes reaching the plama are transported by lymph Elow and taking in account that the passage time of lymph Erom heart to plasma is estimated to be only 20 minutes [Spleckermann, 1979] one may take the view that cardiac enzymes are released directly in the bloodstream.

The incomplete and highly variable recovery in plasma of CK depleted from the heart observed in experimental infarctions in the dog evokes the question which factors may prevent enzymes released from cardiac cells to reach the circulation. In the earlier mentioned study of Clark et al. the degradation of CK in lymph was proposed as an explanation of this small fraction of $\mathrm{CK}$ reaching the plasma. In view of the small amount of enzyme transported by lymph and the short passage time of the lymph this seems an improbably mechanism.

In a recent study it was demonstrated that coronary ligation in the dog resulted in a much more rapid and complete disappearance of $C K$ from the infarcted tissue then found for $L D$ and myoglobin [Johnson, 1981]. From the strictly parallel loss of LD (M $=136000)$ and of myoglobin $(M=17600)$ observed in this study the conclusion was drawn that the extra loss of $\mathrm{CK}$ is due to local inactivation and not caused by a faster efflux of $\mathrm{CK}$ from the tissue. The finding that incubation in vitro of dog myocardial tissue also resulted in a fast loss of CK activity from the tissue in contrast to a nearly constant level of LD supported this conclusion. Such a susceptibility of $\mathrm{CK}$ for local degradation following from both examples above mentioned is also apparent from experiments where a preparation of heart $\mathrm{CK}$ and cytosolic AST was injected in the skeletal muscle of a dog and where only 508 of $\mathrm{CK}$ appeared in plasma in contrast to a 1008 recovery of AST [Visser, 1981a; van Dieijen-Visser, 1981]. In view of these findings it is regrettable that the serum entrance ratio, 
i.e. the fraction of enzyme lost from the heart that appears in the bloodstream, is only studied for the case of CK in the aog. By such studies it is now well documented that the serum entrance ratio of $C K$ is lower for large massive infarctions than for smaller or scattered infarctions [Cairns, 1978; Swain; 1980] and that recovery in plasma of $\mathrm{CK}$ lost from the heart may vary from $15 \%$ [She11, 1971], after correction for the anomalous distribution volume, to $73 \%$ for scattered infarctions [Cairns, 1978], after recalculation of the data with the elimination constant FCR $=0.30 \mathrm{~h}^{-1}$.

It is questionable whether these results for CK after experimental infarction in the dog can be extrapolated to other enzymes and other species. For instance the fast loss of enzyme activity of $C K$ in heart $t$ issue when incubated in vitro was found for the dog but not for the rabbit nor for autopsies of human hearts [Johnson, 1981; Kjekshus, 1970; Van der Laarse, 1980] and the susceptibility of CK for denaturation in lymphe is not found for LDH [Robison, 1975]. 
APPENDIX A

DERIVATION OF THE MATHEMATICAL EXPRESSIONS USED IN CHAPTER 2

\section{A. 1 Compartmental analysis}

The dynamical state of the variables of a (linear) compartmental system is determined by a set of Iinear differential equations:

$$
\frac{d}{d t} x_{i}=\sum_{j=1}^{n} a_{i j} x_{j}+f_{i} ; \begin{aligned}
& i=1, \ldots, n \\
& j=1, \ldots, n
\end{aligned}
$$

with

$t$ - the time expressed in arbitrary units

$x_{i}$ - the size of. the $i-t h$ pool expressed in arbitrary units

$a_{i j}$ - the fractional transfer rate constant of transport from pool $j$ to pool $i(i \neq j)$

$a_{i i}$ - the total fractional transfer rate constant of transport from pool i to the other pools including the irreversible removal from the system at a rate $\mathrm{a}_{0 i}$. i.e. $a_{i 1}=-\sum_{\substack{j=0 \\ j \neq i}}^{n} a_{j i}$

$f_{i}$ - the extraneous input in pool i per unit of time.

Using the notation: $x=\left(x_{1}, \ldots, x_{n}\right)$

$$
\begin{aligned}
& E=\left(E_{1}, \ldots, E_{n}\right) \\
& A=\left(a_{i j}\right)
\end{aligned}
$$

the set of equations (A.I) equals

$$
\frac{d}{d t} x=A x+f
$$

The general solution of these equations with given initial values $x(0)=x_{0}$ is given by.

$$
x(t)=e^{A t} x_{0}+\int_{0}^{t} e^{-A(t-\tau)} f(\tau) d \tau
$$


In the general case that the matrix A has distinct eigenvalues i. e.

$$
A=P A P^{-1}
$$

with

$\Lambda$ - the diagonal matrix of the eigenvalues $\lambda_{1}, i=1, \ldots, n$

P - the matrix formed by the corresponding eigenvectors as columns.

the exponential $e^{A t}$ can be expressed as

$$
\mathrm{e}^{\mathrm{At}}=\mathrm{P} \mathrm{e}^{\Lambda t} \mathrm{P}^{-1}=\mathrm{P}\left\{\begin{array}{ll}
\mathrm{e}^{\lambda_{1} \mathrm{t}} & \\
\phi & \phi \\
& \mathrm{e}^{\lambda_{\mathrm{n}} t}
\end{array}\right\} \mathrm{P}^{-1}
$$

The unit impulse response upon input in the first compartment is then given by

$$
x_{b}(t)=p e^{\Lambda t} P^{-1}\left\{\begin{array}{l}
1 \\
0 \\
0
\end{array}\right\}
$$
1. e.

$$
\begin{aligned}
& x_{b}(t)=Q\left|\begin{array}{l}
e^{\lambda_{1} t} \\
e_{\lambda_{n} t}^{\lambda}
\end{array}\right| \text {, with } Q_{i j}=P_{i j}\left(P^{-1}\right)_{j 1} \\
& x_{b i}(t)=\sum_{j=1}^{n} Q_{i j} e^{\lambda j t}=\sum_{j=1}^{n} P_{i j}\left(P^{-1}\right)_{j 1} e^{\lambda j t}
\end{aligned}
$$

On the other hand if the (observed) unit impulse response is resolved in exponential terms:

$$
x_{b}(t)=Q\left|\begin{array}{l}
e^{\lambda_{1} t} \\
e^{\lambda_{n} t}
\end{array}\right|=Q e_{t}
$$

then

$$
\frac{d}{d t} x_{b}(t)=Q M e_{t}=A Q e_{t}
$$

As the family of vectors $e_{t}, t \geq 0$, spans the $R^{n}$ this last relation implies 


$$
A Q=Q A
$$

For non-singular matrices $Q$ the coefficient matrix $\mathbb{A}$ thus can be expressed explicitely in terms of the observed $Q$ and $\Lambda$ :

$$
A=Q \wedge Q^{-1}
$$

The observation of all components of $x_{b}$ enables one to determine uniquely the coefficient matrix $\mathbb{A}$. This in contrast to the situation that only a projection (of rank less than $n$ ) of the state variables is observed, say

$$
y_{b}(t)=B x_{b}(t) \text {, with rank }(B)<n
$$

Then there exists a nontrivial family of transformations $T$ with the property

$$
\mathrm{BT}=\mathrm{B}
$$

which implies for any $Q$ compatible with the observations, i.e.

$$
B Q e_{t}=B x_{b}(t)=y_{b}(t)
$$

that

$$
\text { BTQe }_{t}=y_{b}(t)
$$

i.e TQ is equally compatible with the observations. Using (A.3) we then find that the family coefficient matrices

$$
A_{\mathrm{TT}}=\mathrm{TQ} \wedge Q^{-1} \mathrm{~T}^{-1}
$$

is compatible with the observed data $Y_{b}(t)$ for all $T$ satisfying (A.5).

Given restrictions on the observability that cause the undeterminacy of the rate constant matrix A from the observed unit 
impulse response upon input in one of the compartments, the examination of the set of responses upon input in severa 1 com partments may resolve the unidentifiability IDelforge, 1981; Norton, 19801 .

often, however such an approach is not practically, e.g. because only one of the compartments is attainable for input or because of the fuzzy definition of the other compartinents. In such cases the requirement that the transfer rate constant matrix must be physically realisabie, i.e.

$$
a_{i j} \leqq-\sum_{\substack{j \neq 1 \\ j \neq 1}}^{n} a_{j i} \text { and } a_{i j} \geq 0
$$

together with the representation (A.6) of the set of compatible matrices $A_{T}$ often leads to rather narrow bounds for the rate constants [Lewallen, 1959a,b].

An alternative is of course to invoke a priori assumptions that restrict the rate constant matrix, e.g. $a_{i j}=0$ for specified values of $i$ and $j$ [Grewal1, 1976; Walter, 1981; Mathews, 1956].

For the explicit calculation of solutions of the set of linear differential equations (A.1) often proceeds more conveniently by using the Laplace transformation than by the above described formalism. Applying the Laplace transformation to both sides of (A.1) we find, cf. Table A.1:

i.e.

$$
s \bar{x}(s)-x_{0}=A \bar{x}(s)+\bar{f}(s)
$$

$$
\bar{x}(s)=(s I-A)^{-1}\left(x_{0}+\bar{E}(s)\right)
$$

From Cramers rule it follows that

$$
(s I-A)^{-1}=\left(B_{0}+B_{1} s+\cdots+B_{n-1} s^{n-1}\right) / \operatorname{det}(s I-A)
$$

with $n \times n$ matrices $B_{j}, j=0, \ldots, n-1$, depending on $A_{\text {. }}$ BY partial fraction expansion this last formula is reduced to 


$$
(s I-A)^{-1}=\frac{C_{1}}{s-\lambda_{1}}+\ldots .+\frac{C_{n}}{s-\lambda_{n}}
$$

By inversion of the Laplace transformation, cf. Table A.1, it is found that $x(t)$ equals:

$$
x(t)=\sum_{j=1}^{n} e^{\lambda j t} \cdot c_{j} x_{0}+\sum_{j=0}^{n} \int^{t} e^{\lambda j(t-\tau)} c_{j} f(\tau) d \tau
$$

which is essentially equivalent to equation (A.2).

Table A.1. Properties of the Laplace transformation

$$
\begin{array}{rlrl}
\int_{-\infty}^{\infty}|f(t)| e^{-s t} d t<\infty & & \rightarrow \bar{f}(s)=\int_{0}^{\infty} e^{-s t} f(t) d t ; R e s \geq s_{0} \\
\int_{1}^{\infty}|\vec{f}(c+i s)| d s<\infty & \rightarrow f(t)=\frac{1}{2 \pi} \int_{-\infty}^{\infty} \bar{f}(c+i s) e^{t(c+i s) d s} \\
g(t)=\int_{0}^{t} f(\tau) d \tau & \leftrightarrow f_{1}(t)=f_{2}(t) \\
g(t)=\frac{d}{d t} f(t) & \leftrightarrow \bar{g}(s)=\bar{f}(s) / s \\
g(t)=e^{-k t}(s), s>0 & \leftrightarrow \bar{g}(s)=s \bar{f}(s)-f(0) \\
g(t)=\int_{0}^{t} f_{1}(\tau) \text { f } f_{2}(t-\tau) d \tau & \leftrightarrow \bar{g}(s)=\bar{f}_{1}(s) \bar{f}_{2}(s) \\
g(t)=\delta(t)=t h e \text { unit } & \leftrightarrow \bar{g}(s)=1 \\
\text { impulse at } t=0
\end{array}
$$

\section{A. 2 The_two-compartment_model}

The dynamics of the state variables $P(t)$ and $E(t)$ of the general two-compartment model presented in Fig. 2.4 is described by 


$$
\begin{aligned}
& \frac{d}{d t} P=-K_{p} P+E R R+f ; K_{p}=F C R+T E R \\
& \frac{d}{d t} E=T E R P-K_{e} E \quad ; K_{e}=F_{e}+E R R
\end{aligned}
$$

Laplace transformation of this equation gives for $\mathbb{P}(0)=0$ and $E(0)=0$ :

$$
\begin{aligned}
& \left(s+K_{p}\right) \bar{P}(s)=\operatorname{ERR} \bar{E}(s)+\bar{f}(s) \\
& \left(s+K_{e}\right) \bar{E}(s)=\operatorname{TER} \bar{P}(s)
\end{aligned}
$$

which can be solved for $\overline{\mathrm{P}}$ and $\overline{\mathrm{E}}$ :

$$
\begin{aligned}
& \overline{\mathrm{P}}(\mathrm{s})=\overline{\mathrm{f}}(\mathrm{s})\left(\mathrm{s}+\mathrm{K}_{\mathrm{e}}\right) / \mathrm{q}_{2}(\mathrm{~s}) ; \mathrm{q}_{2}(\mathrm{~s})=\left(\mathrm{s}+\mathrm{K}_{\mathrm{p}}\right)\left(\mathrm{s}+\mathrm{K}_{\mathrm{e}}\right)-\mathrm{TER} \text { ERR } \\
& \overline{\mathrm{E}}(\mathrm{s})=\overline{\mathrm{f}}(\mathrm{s}) \mathrm{TER} / \mathrm{q}_{2}(\mathrm{~s})
\end{aligned}
$$

The Laplace transformation of the unit impulse response equals 1 and consequently we obtain for the unit impulse response the expression

$$
\begin{aligned}
& \overrightarrow{\mathrm{P}}(s)=\left(s+K_{e}\right) / q_{2}(s) \\
& \overrightarrow{\mathrm{E}}_{\mathrm{b}}(s)=\operatorname{TER} / \mathrm{q}_{2}(s)
\end{aligned}
$$

Factorization of the denominator $q_{2}(s)$

$$
\left(s+K_{p}\right)\left(s+K_{e}\right)-\operatorname{TER} \operatorname{ERR}=\left(s+k_{1}\right)\left(s+k_{2}\right)
$$

with

$$
k_{1,2}=\frac{1}{2}\left(k_{p}+k_{e} \pm \sqrt{\left(k_{p}+K_{e}\right)^{2}-4\left(k_{p} k_{e}-T E R \text { ERR }\right)}\right)
$$

and subsequent partial fraction expansion results in

$$
\begin{aligned}
& \bar{P}_{b}(s)=\frac{P_{1}}{s+k_{1}}+\frac{P_{2}}{s+k_{2}}=\frac{P_{1}\left(s+k_{2}\right)+P_{2}\left(s+k_{1}\right)}{\left(s+k_{1}\right)\left(s+k_{2}\right)} \\
& \bar{E}_{b}(s)=\frac{E_{1}}{s+k_{1}}+\frac{E_{2}}{s+k_{2}}=\frac{E_{1}\left(s+k_{2}\right)+E_{2}\left(s+k_{1}\right)}{\left(s+k_{1}\right)\left(s+k_{2}\right)}
\end{aligned}
$$


The coefficients $\mathbb{P}_{1}, \mathrm{P}_{2}, \mathrm{E}_{1}$ and $\mathrm{E}_{2}$ are determined by comparison of the numerators of $(A .8)$ and $(A .10)$

$$
\begin{aligned}
& \mathrm{P}_{1}\left(s+\mathrm{k}_{2}\right)+\mathrm{P}_{2}\left(s+\mathrm{k}_{1}\right)=s+\mathrm{k}_{\mathrm{e}}: \mathrm{P}_{1}=\frac{\mathrm{k}_{1}-\mathrm{K}_{e}}{\mathrm{k}_{1}-\mathrm{k}_{2}} ; \mathrm{P}_{2}=\frac{\mathrm{k}_{2}-\mathrm{K}_{e}}{\mathrm{k}_{2}-\mathrm{k}_{1}} \\
& \mathbb{E}_{1}\left(s+\mathrm{k}_{2}\right)+\mathrm{E}_{2}\left(s+\mathrm{k}_{1}\right)=\mathrm{TER}: \mathrm{E}_{1}=\frac{\mathrm{TER}}{\mathrm{k}_{2}-\mathrm{k}_{1}} ; \mathrm{E}_{2}=\frac{\mathrm{TER}}{\mathrm{k}_{1}-\mathrm{k}_{2}}
\end{aligned}
$$

By inversion of the Laplace transformation one obtains

$$
\begin{aligned}
& P_{b}(t)=P_{1} e^{-k_{1} t}+P_{2} e^{-k_{2} t} \\
& E_{b}(t)=E_{1} e^{-k_{1} t}+E_{2} e^{-k_{2} t}
\end{aligned}
$$

with $k_{1}$ and $k_{2}$ given by (A.9) and $P_{1}, P_{2}, E_{1}$ and $E_{2}$ by (A.11). Reversely the circulatory parameters FCR, TER, ERR and FCR can be calculated from the bi-exponential representation of the unit impulse response (A.12) by equating (A.10) to (A.8):

Equality of the denominators

Equality of the numerators

$$
\begin{aligned}
& \mathrm{K}_{\mathrm{e}}=\mathrm{P}_{1} \mathrm{k}_{2}+\mathrm{P}_{2} \mathrm{k}_{1} \\
& \mathrm{TER}=\mathrm{E}_{1} \mathrm{k}_{2}+\mathrm{E}_{2} \mathrm{k}_{1}
\end{aligned}
$$

If only observations of $\mathbb{P}_{b}(t)$ are available and thus the equation (A.13b) is lacking the value of $F_{C R}$ remains undetermined. Assuming a value $\mathrm{FCR}_{\mathrm{e}}=0$ we find

$$
\begin{aligned}
& \text { (A.13a) ERR' }=\mathrm{P}_{1} \mathrm{k}_{2}+\mathrm{P}_{2} \mathrm{k}_{1} \\
& \text { (A.13C) } \\
& \mathrm{TER}^{\prime}=-\frac{\left(\mathrm{k}_{1}-\mathrm{ERR}^{\prime}\right)\left(\mathrm{k}_{2}-\mathrm{ERR}^{\prime}\right)}{E R R^{\prime \prime}}=\frac{\mathrm{P}_{1} \mathrm{P}_{2}\left(\mathrm{k}_{1}-\mathrm{k}_{2}\right)^{2}}{\mathrm{P}_{1} \mathrm{k}_{2}+\mathrm{P}_{2} \mathrm{k}_{1}} \\
& \text { (A. 13d) } \\
& \mathrm{FCR}^{\prime}=\frac{\mathrm{k}_{1} \mathrm{k}_{2}}{\mathrm{ERR}^{\prime}}=\frac{1}{\mathrm{P}_{1} / \mathrm{k}_{1}+\mathrm{P}_{2} / \mathrm{k}_{2}}
\end{aligned}
$$

The relation between the primed parameters (A.14) found by setting the value of $\mathrm{FCR}_{e}=0$ and the values of FCR, TER and ERR cal- 
culated from (A.13) by assuming another arbitrary value for FCR , with $0<F C R e<R_{e}$, is given by:

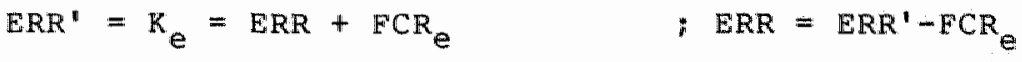

$$
\begin{aligned}
& \mathrm{TER}^{\prime \prime}=\frac{\mathrm{TER}^{\prime \prime} \mathrm{ERR}^{\prime}}{\mathrm{ERR}^{\prime \prime}}=\operatorname{TER}\left(\frac{\mathrm{ERR}}{\mathrm{ERR}+\mathrm{ECR}}\right) ; \operatorname{TER}_{\mathrm{R}}=\mathrm{TER}^{\prime}\left(\frac{\mathrm{ERR}}{\mathrm{ERR}^{\prime}-\mathrm{FCR}_{\mathrm{e}}}\right) \\
& \mathrm{FCR}^{\prime}=\frac{\mathrm{k}_{1} \mathrm{k}_{2}}{\mathrm{ERR}^{\prime}}=\mathrm{FCR}^{\prime}+\left(\frac{\mathrm{TER}}{\mathrm{ERR}+\mathrm{ECR}}\right) \mathrm{FCR}_{\mathrm{e}} ; \mathrm{FCR}=\mathrm{FCR}^{\prime}-\left(\frac{\mathrm{TER}}{\mathrm{ERR}^{\prime}-\mathrm{FCR}_{\mathrm{e}}}\right) \mathrm{ECR}_{\mathrm{e}}
\end{aligned}
$$

Although the parameters FCR, TER and ERR remain undetermined when $E_{b}(t)$ is not observed and with unknown FCR, 1 t is often possible to derive rather narrow ranges for the circulatory parameters from the requirement that the model must be physically interpretable, i.e. ECR $>0$, TER $>0$, ERR $>0$.

The inequalities imply

$$
0<\mathrm{FCR}_{\mathrm{e}}<\frac{\mathrm{ERR}^{\prime}}{\left(\mathrm{FCR}{ }^{\prime \prime}+\mathrm{TER}^{\prime \prime}\right)} \mathrm{FCR}^{\prime}
$$

$\frac{\mathrm{TER}^{\prime}}{\mathrm{ECR}^{\prime}+\mathrm{T}^{\prime} \mathrm{ER} \mathrm{R}^{\prime}} \quad \mathrm{ERR}^{\prime \prime}<\mathrm{ERR}<\mathrm{ERR}$ '

TER $^{*}<$ TER $<$ TER $^{\prime \prime}+$ FCR

Hence for values of FCR' small compared to 'TER' and ERR' accurate estimations of TER and ERR are obtained.

\section{A. 3 The_three-compartment_mamm 1 lary_model}

The dynamics of the state variable of the mammilary model, presented in Fig. 2.5 , is governed by the differential equations:

$\frac{d}{d t} P=-K_{p} P+E R R_{1} E_{1}+E R R_{2} E_{2}+E(t)$

$\frac{\mathrm{d}}{\mathrm{dt}} \mathrm{E}_{1}=\mathrm{TER}_{1} \mathrm{P}-\mathrm{ERR}_{1} \mathrm{E}_{1} ; \mathrm{K}_{\mathrm{p}}=\mathrm{FCR}+\mathrm{TER}_{1}+\mathrm{TER}_{2}$

$\frac{d}{d t} E_{2}=\operatorname{TER}_{2} P-E_{2} E_{2}$ 
Laplace transfornation of these equations with $f(t)$ equal to the unit impulse response, i.e. $f(t)=\hat{f}(t)$, and with $P(0)=0$, $E_{1}(0)=0$ and $E_{2}(0)=0$ results in:

$$
\begin{aligned}
& \left(s+R_{p}\right) \bar{p}_{b}(s)=\operatorname{ERR}_{1} \bar{E}_{b 1}(s)+E_{2} R_{2} \bar{E}_{b 2}(s)+1 \\
& \left(s+E_{1}\right) \bar{E}_{b 1}(s)=\operatorname{TER}_{1} \overline{\mathbb{P}}_{\mathrm{b}}(s) \\
& \left(s+\mathbb{E R R}_{2}\right) \bar{E}_{\mathrm{b} 2}(s)=\operatorname{TER}_{2} \overline{\mathrm{P}}_{\mathrm{b}}(s)
\end{aligned}
$$

Solving these equations for $\overline{\mathrm{P}}_{b}, \overline{\mathrm{E}}_{b}$ and $\overline{\mathrm{E}}_{b}$ one obtains:

$$
\begin{aligned}
& \overline{\mathrm{P}}_{\mathrm{b}}(\mathrm{s})=\left(\mathrm{s}+\mathrm{ERR}_{1}\right)\left(\mathrm{s}+\mathrm{ERR}_{2}\right) / \mathrm{q}_{3}(\mathrm{~s}) \\
& \overline{\mathrm{E}}_{\mathrm{b} 1}(\mathrm{~s})=\operatorname{TER}_{1}\left(s+\mathrm{ERR}_{2}\right) / \mathrm{q}_{3}(\mathrm{~s}) \\
& \overline{\mathrm{E}}_{\mathrm{b} 2}(\mathrm{~s})=\operatorname{TER}_{2}\left(s+\mathrm{ERR}_{1}\right) / \mathrm{q}_{3}(\mathrm{~s})
\end{aligned}
$$

with

$q_{3}(s)=\left(s+K_{p}\right)\left(s+E R R_{1}\right)\left(s+E R R_{2}\right)-T E R_{1} E R R_{1}\left(s+E R R_{2}\right)-T E R_{2} E_{2}\left(s+E R R_{1}\right)$

Let $-k_{1},-k_{2},-k_{3}$ be the (real) roots of $q_{3}(s)=0$, i.e.

$$
q_{3}(s)=\left(s+k_{1}\right)\left(s+k_{2}\right)\left(s+k_{3}\right)
$$

Then partial fraction expansion of (A.15) results in

$$
\begin{aligned}
& \bar{P}_{b}(s)=\frac{P_{1}}{s+k_{1}}+\frac{P_{2}}{s+k_{2}}+\frac{P_{3}}{s+k_{3}}= \\
& \frac{P_{1}\left(s+k_{2}\right)\left(s+k_{3}\right)+\mathbb{P}_{2}\left(s+k_{1}\right)\left(s+k_{3}\right)+P_{3}\left(s+k_{1}\right)\left(s+k_{2}\right)}{\left(s+k_{1}\right)\left(s+k_{2}\right)\left(s+k_{3}\right)} \\
& \overrightarrow{\mathrm{E}}_{\mathrm{bl}}(s)=\frac{\mathrm{E}_{11}}{s+\mathrm{k}_{1}}+\frac{\mathrm{E}_{12}}{s+\mathrm{k}_{2}}+\frac{\mathrm{E}_{13}}{s+\mathrm{k}_{3}}= \\
& \frac{E_{11}\left(s+k_{2}\right)\left(s+k_{3}\right)+E_{12}\left(s+k_{1}\right)\left(s+k_{3}\right)+E_{13}\left(s+k_{1}\right)\left(s+k_{2}\right)}{\left(s+k_{1}\right)\left(s+k_{2}\right)\left(s+k_{3}\right)} \\
& \vec{E}_{b 2}(s)=\frac{E_{21}}{s+k_{1}}+\frac{E_{22}}{s+k_{2}}+\frac{E_{23}}{s+k_{3}}= \\
& \frac{E_{21}\left(s+k_{2}\right)\left(s+k_{3}\right)+E_{22}\left(s+k_{1}\right)\left(s+k_{3}\right)+E_{23}\left(s+k_{1}\right)\left(s+k_{2}\right)}{\left(s+k_{1}\right)\left(s+k_{2}\right)\left(s+k_{3}\right)}
\end{aligned}
$$


with coefficients $\mathrm{P}_{1}, \mathrm{E}_{11}$ and $\mathrm{E}_{21}, i=1,2,3$, determined by the requirement that the denominators of (A.15) and (A.16) must be equal at $s=-k_{1},-k_{2}$ and $-k_{3}$ respectively:

$$
\begin{aligned}
& P_{1}=\frac{\left(E R R_{1}-k_{1}\right)\left(E R R_{2}-k_{1}\right)}{\left(k_{2}-k_{1}\right)\left(k_{3}-k_{1}\right)} ; E_{11}=\frac{\operatorname{TER}_{1}\left(E R R_{2}-k_{1}\right)}{\left(k_{2}-k_{1}\right)\left(k_{3}-k_{1}\right)} \\
& E_{21}=\frac{T_{E R}\left(E R R_{1}-k_{1}\right)}{\left(k_{2}-k_{1}\right)\left(k_{3}-k_{1}\right)} ; P_{2}=\frac{\left(E R R_{1}-k_{2}\right)\left(E R R_{2}-k_{2}\right)}{\left(k_{1}-k_{2}\right)\left(k_{3}-k_{2}\right)} \\
& E_{12}=\frac{\operatorname{TER}_{1}\left(\operatorname{ERR}_{2}-\mathrm{k}_{2}\right)}{\left(\mathrm{k}_{1}-\mathrm{k}_{2}\right)\left(\mathrm{k}_{3}-\mathrm{k}_{2}\right)} ; \mathrm{E}_{22}=\frac{\mathrm{TER}_{2}\left(\mathrm{ERR}_{1}-\mathrm{k}_{2}\right)}{\left(\mathrm{k}_{1}-\mathrm{k}_{2}\right)\left(\mathrm{k}_{3}-\mathrm{k}_{2}\right)} \\
& \mathrm{P}_{3}=\frac{\left(\mathrm{ERR}_{1}-\mathrm{k}_{3}\right)\left(\mathrm{ERR}_{2}-\mathrm{k}_{3}\right)}{\left(\mathrm{k}_{1}-\mathrm{k}_{3}\right)\left(\mathrm{k}_{2}-\mathrm{k}_{3}\right)} ; \mathrm{E}_{13}=\frac{\mathrm{TER}_{1}\left(\mathrm{ERR}_{2}-\mathrm{k}_{3}\right)}{\left(\mathrm{k}_{1}-\mathrm{k}_{3}\right)\left(\mathrm{k}_{2}-\mathrm{k}_{3}\right)} \\
& E_{23}=\frac{T_{2}\left(E R R_{1}-k_{3}\right)}{\left(k_{1}-k_{3}\right)\left(k_{2}-k_{3}\right)}
\end{aligned}
$$

Thus inversion of the Laplace transformation gives:

$$
\begin{aligned}
& \mathbb{P}_{b}(t)=P_{1} e^{-k_{1} t}+P_{2} e^{-k_{2} t}+P_{3} e^{-k_{3} t} \\
& E_{b 1}(t)=E_{11} e^{-k_{1} t}+E_{12} e^{-k_{2} t}+E_{13} e^{-k_{3} t} \\
& E_{b 2}(t)=E_{21} e^{-k_{1} t}+E_{22} e^{-k_{2} t}+E_{23} e^{-k_{3} t}
\end{aligned}
$$

with $P_{i}, E_{1 i}$ and $E_{2 i}$ given by (A.17).

The calculation of the circulatory parameters $F C R, T E R_{1}, T E R_{2}$, $E R R_{1}$ and $E R R_{2}$ from the observed three-exponential decay of the plasma pool after an unit bolus injection

$$
P_{b}(t)=P_{1} e^{-k_{1} t}+P_{2} e^{-k_{2} t}+P_{3} e^{-k_{3} t}
$$

proceeds by comparison of the formula's (A.15) and (A.16). The equality of the denominators implies

$$
\mathrm{ERR}_{1,2}=1 / 2\left(\mathrm{H}_{1} \pm \sqrt{\mathrm{H}_{1}^{2}-4 \mathrm{H}_{2}}\right)
$$

with

$$
\begin{aligned}
& \mathrm{H}_{1}=\mathrm{P}_{1}\left(\mathrm{k}_{2}+\mathrm{k}_{3}\right)+\mathrm{P}_{2}\left(\mathrm{k}_{1}+\mathrm{k}_{3}\right)+\mathrm{P}_{3}\left(\mathrm{k}_{1}+\mathrm{k}_{2}\right)=\mathrm{ERR}_{1}+\mathrm{ERR}_{2} \\
& \mathrm{H}_{2}=\mathrm{P}_{1}\left(\mathrm{k}_{2} \mathrm{k}_{3}\right)+\mathrm{P}_{2}\left(\mathrm{k}_{1} \mathrm{k}_{3}\right)+\mathrm{P}_{3}\left(\mathrm{k}_{1} \mathrm{k}_{2}\right)=\mathrm{ERR}_{1} \mathrm{ERR}_{2}
\end{aligned}
$$


By equating the numerators of (A.15) and $(A .16)$ for $s=-E R R_{1}$, $-\mathrm{ERR}_{2}$ and $\mathrm{s}=0$ one obtains:

$$
\begin{aligned}
-\mathrm{TER}_{1} \mathrm{ERR}_{1}\left(\mathrm{ERR}_{2}-\mathrm{ERR}_{1}\right) & =\left(k_{1}-\mathrm{ERR}_{1}\right)\left(k_{2}-\mathrm{ERR}_{1}\right)\left(k_{3}-\mathrm{ERR}_{1}\right) \\
-\mathrm{TER}_{2} \mathrm{ERR}_{2}\left(\mathrm{ERR}_{1}-\mathrm{ERR}_{2}\right) & =\left(k_{1}-\mathrm{ERR}_{2}\right)\left(k_{2}-\mathrm{ERR}_{2}\right)\left(k_{3}-\mathrm{ERR}_{2}\right) \\
\mathrm{ECR} \mathrm{ERR}_{1} \mathrm{ERR}_{2} & =k_{1} k_{2} k_{3}
\end{aligned}
$$

Solving these expressions for $\mathrm{TER}_{1}, \mathrm{TER}_{2}$ and $\mathrm{FCR}$ results in:

$$
\begin{aligned}
& \operatorname{TER}_{1}=\left(k_{1}-E R R_{1}\right)\left(k_{2}-E R R_{1}\right)\left(k_{3}-E R R_{1}\right) / E R R_{1}\left(E R R_{1}-E R_{2}\right) \\
& \operatorname{TER}_{2}=\left(k_{1}-E R R_{2}\right)\left(k_{2}-E R R_{2}\right)\left(k_{3}-E R R_{2}\right) / E R R_{2}\left(E R R_{2}-E R_{1}\right) \\
& F C R=1 /\left(P_{1} / k_{1}+P_{2} / k_{2}+P_{3} / k_{3}\right)
\end{aligned}
$$

\section{A.4 Quantifíication_of_ingut_into_o_plasma}

The problem of obtaining the rate of input into the plasma $f(t)$ from the observed plasma response curve is encountered in the field of enzymatic infarct sizing and also in the study of drug delivery to the circulation [Cutler, 1978; Veng Pederson, 1978]. The calculation of $f(t)$ or of the total quantity $A(t)$ that entered the circulation up to time $t$ can be performed on basis of compartmental models once the parameters are identified. Another approach assumes the additivity and the time-invariance of the system response upon an input $\mathbb{f}(t)$. Then the response $P(t)$ may be expressed in terms of the unit impulse response $P_{b}(t)$, cf. Formu1. 2.12:

$$
P(t)=\int_{0}^{t} f(\tau) P_{b}(t-\tau) d
$$

Laplace transformation of this formula results in

$$
\overline{\mathrm{P}}(\mathrm{s})=\overline{\mathrm{f}}(\mathrm{s}) \overline{\mathrm{P}}_{\mathrm{b}}(\mathrm{s})
$$

and formally one may express $\bar{f}(s)$ in $\vec{P}(s)$ and $\bar{P}_{b}(s)$ : 


$$
\bar{f}(s)=\bar{P}(s) / \bar{P}_{b}(s)
$$

By inversion of the Laplace transformation one obtains an expression for $f(t)$.

In the frequently encountered situation, cf. Appendix A.1, that $P_{b}(t)$ is equal to a sum of exponentials:

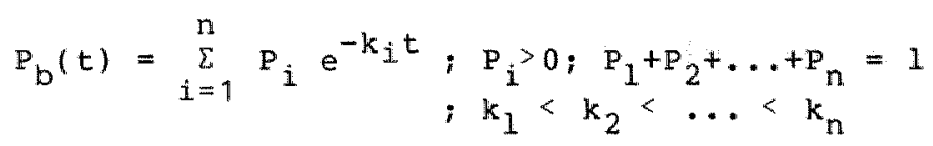

one may derive an explicit expression for the input $f(t)$. Starting from (A.19-20) one obtains

$$
\begin{aligned}
\bar{f}(s) & =\left\{\sum_{i=1}^{n} \frac{\mathrm{P}_{i}}{s+k_{i}}\right\}^{-1} \overline{\mathrm{P}}(s) \\
& =\left(\prod_{i=1}^{n}\left(s+k_{i}\right)\right) /\left(\sum_{i=1}^{n} \mathrm{P}_{i} \prod_{\substack{j=1 \\
j \neq 1}}^{n}\left(s+k_{j}\right)\right) \bar{P}(s)
\end{aligned}
$$

Hence the cumulative input $A(t)=\int_{0}^{t} f(\tau) d \tau$ equals

$$
\begin{aligned}
& \overline{\mathrm{A}}(\mathrm{s})=\frac{1}{\mathrm{~s}} \overline{\mathrm{f}}(\mathrm{s}) \\
& =\left(\prod_{i=1}^{n}\left(s+k_{i}\right)\right) /\left(s \sum_{i=1}^{n} P_{i} \prod_{\substack{j=1 \\
j \neq i}}^{n}\left(s+k_{j}\right)\right) \bar{P}(s)
\end{aligned}
$$

The denominator in this formula is a $n$-th degree polynomial $\bar{q}(s)$ with the properties:

$$
\bar{q}(0)=0, \operatorname{sign}\left(\bar{q}\left(-k_{j}\right)\right)=(-1)^{j}
$$

and thus possesses $n$ distinct real roots $-e_{0}, \ldots,-e_{n-1}$

$$
e_{0}=0 ; k_{j}<e_{j}<k_{j+1} ; j=1, \ldots, n-1
$$

Hence $\bar{q}(\mathbf{s})$ can be factorized $\left(\sum_{i=1}^{n} p_{i}=1\right)$

$$
\bar{q}(s)=\prod_{j=0}^{n-1}\left(s+e_{j}\right)
$$


By partial fraction expansion of (A.21) one finds:

$$
\overline{\mathrm{A}}(s)=\left(1+\sum_{j=0}^{\mathrm{n}-1} \mathbb{A}_{j} /\left(s+e_{j}\right)\right) \overline{\mathrm{P}}(\mathrm{s})
$$

with coefficients $\mathbb{A}_{j}$ satisfying

$$
\prod_{j=0}^{n-1}\left(s+e_{j}\right)+\sum_{j=0}^{n-1} A_{j} \prod_{m=0}^{n-1}\left(s+e_{m}\right)=\prod_{j=1}^{n}\left(s+k_{j}\right)
$$

The coefficients $A_{j}$ are now determined by insertion of $s=-e_{j}$ " $j=0, \ldots, n-1$

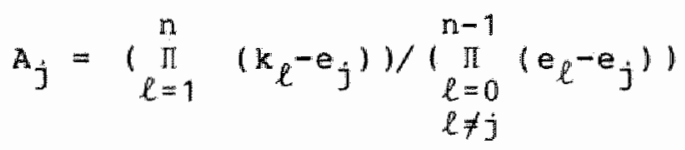

Thus, using $e_{0}=0$, we find for $A(t)$

$A(t)=P(t)+A_{0} \int_{0}^{t} P(\tau) d \tau+\sum_{j=1}^{n-1} A_{j} \int_{0}^{t} e^{-e_{j}(t-\tau)} P(\tau) d \tau \quad(A .24)$ with: $\quad-e_{j}, j=0, \ldots, n-1$ the $n$ zero's of $\bar{q}(s)=0$ and $e_{0}=0$ $A_{j}$ given by $(A .23)$.

For mono-, bi- and tri-exponential responses $P_{b}(t)$ upon a bolus injection the resulting formula's are presented in Table A.2.

The interpretation of these formulas in terms of compartmental models is easily obtained; e.g. for the biphasic response the simplified two-compartmental model can be used:

The curnulative input necessarily equals

$$
A(t)=P(t)+E(t)+D(t)
$$

with - $P(t)$ the amount of protein still present in plasma

- $E(t)$ the amount of protein in the extravascular pool

- $D(t)$ the amount of protein already degraded.

By using the equation (2.4)

$$
\frac{d}{d t} E(t)=\operatorname{TER} P(t)-\operatorname{ERR} E(t) ; E(0)=0
$$


E(t) may be expressed as

$$
E(t)=\operatorname{TER} \int_{0}^{t} e^{-\operatorname{ERR}(t-\tau)} \mathrm{P}(\tau) \mathrm{d \tau}
$$

and the quantity $D(t)$ is simply equal to

$$
D(t)=\mathbb{E C R} \int_{0}^{t} \mathrm{P}(\tau) \mathrm{d \tau}
$$

Hence we have

$$
Q(t)=P(t)+F C R \int_{0}^{t} P(\tau) d \tau+T E R \int_{0}^{t} e^{-E R R(t-T)} P(T) d T
$$

and we see the correspondency

$$
\begin{aligned}
& A_{0}=\text { FCR } \\
& A_{1}=\text { TER } \\
& e_{1}=\text { ERR }
\end{aligned}
$$

between the formula from the Table (A.2) and the two-compartment model $(2.4)$. 
Table A.2 Quantification of the cumulative input $A(t)$ into the plasma; calculation of $A(t)$ from the measured plasma response $\mathrm{P}(\mathrm{t})$ on bases of exponential analysis of the unit impulse response $\mathrm{P}_{\mathrm{b}}(t)$.

Monophasic response:

$$
\begin{aligned}
& P_{b}(t)=P_{1} e^{-k_{1} t} \\
& P(t)=\int_{0}^{t} P_{1} e^{-k_{1}(t-\tau)} f(\tau) d \tau \\
& \left(P_{1}=1\right) \\
& A(t)=\int_{0}^{t} f(\tau) d \tau=P(t)+A_{0} \int_{0}^{t} P(\tau) d \tau ; \quad A_{0}=k_{1}
\end{aligned}
$$

Biphasic cesponse:

$$
\begin{aligned}
& P_{b}(t)=P_{1} e^{-k_{1} t}+P_{2} e^{-k_{2} t} \quad\left(P_{1}+P_{2}=1\right) \\
& P(t)=\int_{0}^{t}\left[P_{1} e^{-k_{1}(t-\tau)}+P_{2} e^{-k_{2}(t-\tau)}\right] f(\tau) d \tau
\end{aligned}
$$

$A(t)=\int_{0}^{t} E(\tau) d \tau=P(t)+A_{0} \int_{0}^{t} P(\tau) d \tau+A_{1} \int_{0}^{t} P(\tau) e^{-e_{1}(t-\tau)} d \tau$ with $e_{1}=P_{1} k_{2}+P_{2} k_{1} ; A_{0}=\frac{k_{1} k_{2}}{e_{1}} ; A_{1}=\frac{\left(k_{1}-e_{1}\right)\left(k_{2}-e_{1}\right)}{\left(-e_{1}\right)}$

Triphasic response:

$$
P_{b}(t)=P_{1} e^{-k_{1} t}+P_{2} e^{-k_{2} t}+P_{3} e^{-k_{3} t} \quad\left(P_{1}+P_{2}+P_{3}=1\right)
$$

$P(t)=\int^{t}\left[P_{1} e^{-k_{1}(t-\tau)}+P_{2} e^{-k_{2}(t-\tau)}+P_{3} e^{-k 3(t-\tau)}\right] f(\tau) d \tau$

$A(t)=\int_{0}^{t} f(\tau) d \tau=P(t)+A_{0} \int^{t} P(\tau) d \tau+A_{1} \int^{t} P(\tau) e^{-e_{1}(t-\tau)} d \tau$

$$
+A_{2} \int_{0}^{t} \mathrm{p}(\tau) \mathrm{e}^{-e_{2}(t-\tau)} \mathrm{d} \tau
$$

$$
\begin{aligned}
& \text { with } e_{1,2}=\frac{1}{2}\left(H_{1} \pm\left(H_{1}-4 H_{2}\right) ; H_{1}=P_{1}\left(k_{2}+k_{3}\right)+P_{2}\left(k_{1}+k_{3}\right)+P_{3}\left(k_{1}+k_{2}\right)\right. \\
& \mathrm{H}_{2}=\mathrm{P}_{1} \mathrm{k}_{2} \mathrm{k}_{3}+\mathrm{P}_{2} \mathrm{k}_{1} \mathrm{k}_{3}+\mathrm{P}_{3} \mathrm{k}_{1} \mathrm{k}_{2} \\
& A_{0}=\frac{k_{1} k_{2} k_{3}}{e_{1} e_{2}} ; A_{1}=\frac{\left(k_{1}-e_{1}\right)\left(k_{2}-e_{1}\right)\left(k_{3}-e_{1}\right)}{\left(-e_{1}\right)\left(e_{2}-e_{1}\right)} ; A_{2}=\frac{\left(k_{1}-e_{2}\right)\left(k_{2}-e_{2}\right)\left(k_{3}-e_{2}\right)}{\left(-e_{2}\right)\left(e_{1}-e_{2}\right)}
\end{aligned}
$$


CEAPTER 3

ANALYSIS OF SIMULTANEOUSLY SAMPLED PLASMA ENZYME ACTIVTTIES

\subsection{Introduction}

The development of a method for the identification of circulatory model parameters by means of an analysis of the plasma curves of several proteins that enter the circulation concurrently is prompted by a number of reasons. As mentioned before, cf. Chapter 2.5, the determination of elimination constants and other circulatory parameters of tissue enzymes from the plasma decay curve after injection of purified enzyme preparations often is complicated by the occurrence of damaged fractions in these preparations. In man furthermore there exists the ethical problem of injection of a foreign tissue protein preparation. These considerations seem to have prevented up to now the application of such kinetic studies for the determination of the circulatory parameters of tissue enzymes in man with a few exceptions mentioned in the previous chapter. In contrast plasma ourves of several enzymes released after organ damage may be obtained in variety of diseases.

The method for the estimation of the circulatory parameters proposed in this chapter can be applied to simultaneously sampled plasma curves of different proteins collected during continuing release. This method depends on the assumption that the time course of release into plasma of the various proteins can be described by the same input function, obviously apart from a scaling proportionality constant. For the validity of this assumption with respect to the release of cytoplasmic cardiac enzymes after AMI much evidence is available, cf. Chapter 2.6.

In section 2 the model for the dynamics of the plasma levels of simultaneously released proteins is presented. This regression problem is complicated by the considerable number of parameters required to represent the input into plasma. In earlier attempts the problem was reduced by the elimination of the unknown input from the regression equations. This was effected by expressing the plasma levels of the second enzyme in terms of a transfer 
Eunction, characterizing the circulatory model for both enzymes, and the plasma levels of the first enzyme [Willems, 1979]. The resulting method, however, did have some disadvantages. Firstly the regresision equations are calculated by using the observed values of the first enzyme, that are contaminated with error. The regressors thus inherently are subject to error. This condition tends to introduce bias in the estimates and troubles the statistical analysis [Seber, 1977]. Furthermore deviations in the plisma curve of the first enzyme are difficult to assess in this set up. Lastly the resulting method permitted only the simultaneous analysis of two different plasma curves.

Therefore it was decided to construct an algorithm for the solution of the full regression problem described in section 3 . This section and the other sections of this chapter are presented such that the main line of reasoning is contained in the text, while the technical details, the more repulsive formula and their derivations are relegated to Appendix $B$. The accuracy of the parameter estimation depends on several factors such as the size of the measurement error of the plasma enzyme activities and the frequency and duration of the sampling. In Section 4 the performance of the estimation procedure is investigated with simulated data sets.

Departures from the underlying assumptions may severely affect the estimated values of the circulatory parameters. In section 5 the effects of some of the more plausible deviations of the model assumptions are investigated.

In the last section of this chapter two simplifications of the general estimation procedure are proposed. In both cases a priori information is used, that must be obtained independently. With the first method it is assumed, that the exchange rates between plasma and the extravascular pool is known, e.g. from experiments with labelled plasma proteins. Then one may estimate the FCR of (rapidly eliminated) enzymes by simultaneous analysis of the plasma activities sampled during 4 days after onset of AMI. The second method allows the determination of FCR of a rapidly eliminated enzyme from an even stil1 shorter auration of sampling of the plasma activities after AMI, but requires kmowledge of the circulatory parameters of HBD that is used as a reference enzyme. 


\subsection{Estimation of the circulatory parameters from simultaneously} sampled plasma curves

If the time course of enzyme release after AMI is identical for all cytoplasmic cardiac enzymes (cf. Section 2.6), the input functions $f_{j}(t)$ describing the input of the $j$-th enzyme all are related to a reference input function $f(t)$ :

$$
\operatorname{rho}_{j} f_{j}(t)=f(t) ; j=1, \ldots, m
$$

with

$$
\begin{aligned}
& f_{j}(t) \text { - the input into plasma of the } j \text {-th enzyme }(U / 1 / h) \\
& r h o_{j} \text { - a scaling factor; rho } j / r h o_{k} \text { equals the ratio of }
\end{aligned}
$$
total release of the $\mathrm{k}-\mathrm{th}$ and $\mathrm{j}-\mathrm{th}$ enzyme

$f(t)$ - describes the time course of the release

In order to define the arbitrary scale of $f(t)$, one of the rho's is fixed, e.g. $r h o_{1}=1$ and thus $f_{1}(t)=f(t)$.

With the representation (3.1) one may express the multiple response of the plasma activities of the m-enzymes by (cf. Section 2.3):

$$
\begin{aligned}
C_{j}(t) & =\left(1 / r h o_{j}\right) \int_{0}^{t} f(\tau) P_{b j}(t-\tau) d \tau \\
& =\left(1 / r h o_{j}\right) \mathbb{P}_{b j}(t)^{*} f(t)
\end{aligned}
$$

where $\mathrm{P}_{\mathrm{bj}}(t)$ represents the unit impulse response of enzyme $j$.

As discussed earlier, it is generally possible to represent the impulse response $P_{b}(t)$ of proteins by a bi-exponential function

$$
P_{b}(t)=P_{1} e^{-k_{1} t}+P_{2} e^{-k_{2} t}
$$

where the parameters $\mathrm{P}_{\ell}, \mathrm{k}_{\ell}$ are related to the values of the circulatory parameters, e.g. FCR, TER and ERR, of the model selected to describe the kinetics of the plasma levels. It was also shown that once the parameters $\mathrm{P}_{\ell}, \mathrm{k}_{\ell}$ characterizing the unit impulse response are known it is possible to calculate the input $f_{j}(t)$ from the observed plasma response curve $C_{j}(t)$. 
The choice of the circulatory model

The simplitied two-compartment model generally allows an adequate description of the kinetics of the plasma levels of proteins in the circulation, cf. Section 2.4. A feature of the data on the dynamics of proteins in the body is the marked range in the values of FCR for the different proteins. While apparently the transcapillary escape rates TER and the extravascular poolsizes $E / P$ do not exhibit much variation for proteins with a molecular welght between 70000 and 160000 . The variation between values of these parameters reported in different studies on the same protein is of the same order as the differences observed for different proteins. This is in agreement with the observations in isolated organ studies where it was found that the molecular size for molecules within this range hardly affects the rate of transfer of these substances from plasma to the extravascular fluids nor the extravascular distribution space.

In view of these data it seems justified to assume identical exchange rates between plasma and the extravascular pool for the various enzymes.

Thus using (3.2) the model depicted in Fig. 3.2 is proposed for the description of the multiple response of the plasma activities of $M$ enzymes after cardiac damage.

The system response upon a general input $f(t)$ is then given by, cf. Chapter 2.3,

$$
c_{j}(t)=\left(1 / r h o_{j}\right)\left(P_{1 j} e^{-k_{1 j}+P_{2 j}} e^{\left.-k_{2 j}\right) * f(t)}\right.
$$

with

$$
\begin{aligned}
& k_{1 j}, k_{2 j}=\frac{1}{2}\left(F C R_{j}+T E R+E R E+\sqrt{\left(F C R_{j}+T E R+E R R\right)^{2}-4 F^{2} R_{j} E R R}\right) \\
& { }_{1 j}=\left(k_{1 j}-E R R\right) /\left(k_{1 j}-k_{2 j}\right) ; P_{2 j}=\left(k_{2 j}-E R R\right) /\left(k_{2 j}-k_{1 j}\right)
\end{aligned}
$$

In Appendix B. 1 it is demonstrated that the simultaneous error free observation of $c_{j}(t),(j=1, \ldots, m)$, allows the unique determination of the circulatory parameters $F_{C R}, T E R$ and ERR of this system even with unknown input $f(t)$. 


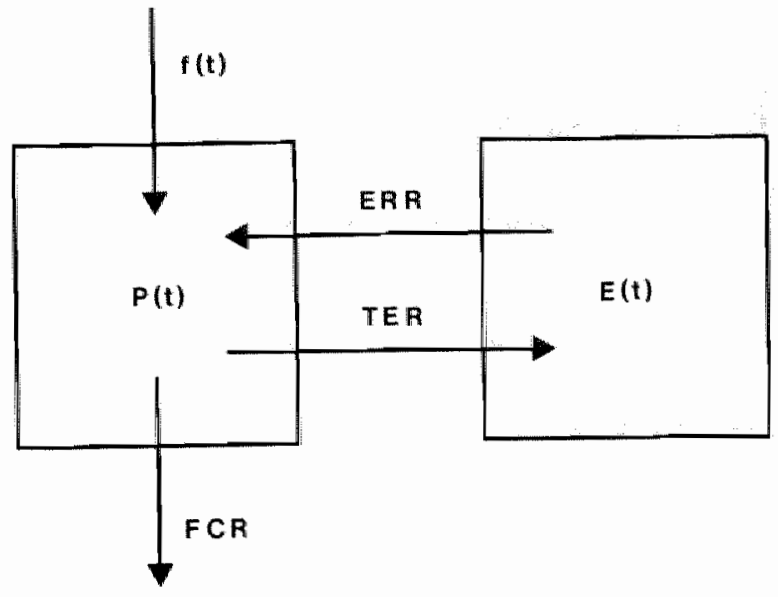

Fig. 3.1 The two compartment madel.

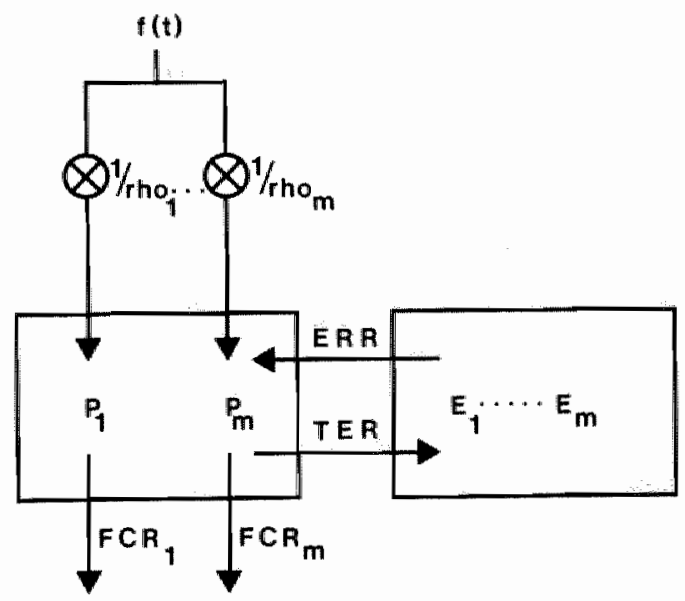

Fig. 3.2 The two-compartment model for simultaneousty released enzymes. 

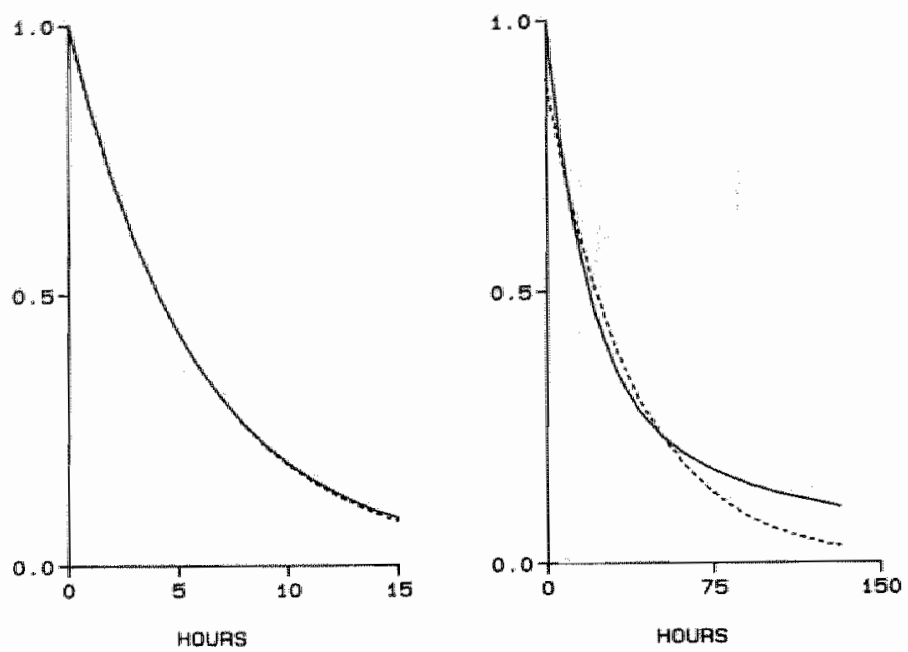

Fig. 3.3 Bi-expontential unit impulse reaponse (solid line) and best ordinary least squares fit by a mono-exponential function (dashed line). Left parel : $\mathbb{F} C R=0.150 ; T E R=0.020 ; E R R=0.020$ Right panel : $E C R=0.020$; TER $=0.020 ; E R R=0.020$

A further simplification of the description of the plasma curves in response to a general input $f(t)$ might be attained by the one-compartment model accounting only for elimination from the plasma and neglecting the distribution over the body fluids other than plasma. For rapidly eliminated proteins, i.e. with a value of FCR which is large compared to the value of TER, such an approximation indeed seems to be acceptable, cf. Table 3.1 and Fig. 3.3, because of the minor error involved in this approximation. Thus it seems attractive to substitute:

for

$$
P_{b j}(t)=e^{-F C R_{j} t}
$$

$$
P_{b j}(t)=\left(P_{1 j} e^{-k_{1 j} t}+P_{2 j} e^{-k_{1 j} t}\right)
$$

in Formula (3.3) in the cases that only rapidly eliminated enzymes are studied. In section 3.5 it is however demonstrated that while the departures arising from this simplification are small for large FCR, the regression of the right hand side of (3.3) on 
Table 3.1 Mono-exponential approximation of the biphasic unit impulse response.

\begin{tabular}{cccccccc}
\multicolumn{8}{c}{$\mathrm{TER}=0.020$} \\
FCR & $\mathrm{P}_{1}$ & $\mathrm{k}_{1}$ & $\mathrm{P}_{2}$ & $\mathrm{k}_{2}$ & $\mathrm{P}_{0}$ & $\mathrm{k}_{0}$ & ReS \\
0.020 & 0.724 & 0.052 & 0.276 & 0.0076 & 0.95 & 0.030 & 0.019 \\
0.050 & 0.890 & 0.077 & 0.110 & 0.0130 & 0.96 & 0.060 & 0.017 \\
0.075 & 0.941 & 0.100 & 0.058 & 0.0150 & 0.98 & 0.088 & 0.008 \\
0.100 & 0.964 & 0.124 & 0.037 & 0.0162 & 0.99 & 0.114 & 0.006 \\
0.150 & 0.983 & 0.173 & 0.017 & 0.0174 & 0.99 & 0.166 & 0.003 \\
0.200 & 0.990 & 0.222 & 0.010 & 0.0180 & 0.99 & 0.216 & 0.003 \\
0.250 & 0.994 & 0.272 & 0.006 & 0.0184 & 0.99 & 0.268 & 0.001 \\
0.300 & 0.996 & 0.321 & 0.004 & 0.0187 & 1.00 & 0.317 & 0.001
\end{tabular}

$\mathrm{TER}=0.040 ; \mathrm{ERR}=0.040$

$\begin{array}{lccccccc}\text { FCR } & P_{1} & k_{1} & P_{2} & k_{2} & P_{0} & k_{0} & \text { Res } \\ 0.050 & 0.765 & 0.112 & 0.235 & 0.0178 & 0.88 & 0.059 & 0.037 \\ 0.075 & 0.842 & 0.132 & 0.158 & 0.0227 & 0.92 & 0.088 & 0.027 \\ 0.100 & 0.890 & 0.154 & 0.110 & 0.0260 & 0.93 & 0.114 & 0.023 \\ 0.150 & 0.941 & 0.200 & 0.059 & 0.0300 & 0.98 & 0.176 & 0.008 \\ 0.200 & 0.964 & 0.248 & 0.036 & 0.0323 & 0.98 & 0.227 & 0.007 \\ 0.250 & 0.976 & 0.296 & 0.024 & 0.0338 & 0.99 & 0.280 & 0.004 \\ 0.300 & 0.983 & 0.345 & 0.017 & 0.0348 & 0.99 & 0.330 & 0.004\end{array}$

The bi-exponential unit impulse response function $\mathbb{P}_{b}(t)$

$$
p_{b}(t)=P_{1} e^{-k_{1} t}+p_{2} e^{-k_{2} t}
$$

is approximated by $\mathrm{P}_{0} \mathrm{e}^{-\mathrm{k}_{0} \mathrm{t}}$ with $\mathrm{P}_{0}$ and $\mathrm{k}_{0}$ determined by an ordinary least squares fit over the time interval $\left(t=0-t_{1}\right)$ with $\mathbb{P}_{b}\left(t_{1}\right)=0.10$.

Res is the mean squared deviation between data and fit. 
data results in a poor determination of the parameters involved and that the small departures give rise to considerable bias in the estimation in consequence of the 111 conditioned regression problem.

Oppositely the refinement of the two-compartment model by allowing different values of $\mathrm{TER}$ and ERR for the different enzymes gives rise to unidentifiability of the circulatory parameters, C. Appendix B.1, and is therefore useless for the determination of the parameters. Thus the restriction of identical values of TER and ERR is essential and it will be necessary to evaluate the effect of departures from this assumption, cf. Chapter 3.5 .

Arother refinement of the model consisting in the introduction of different extravascular pools, with separate values for the exchange rates with plasma, i.e. the description of the plasma response by means of Mathews mamilary model will cause analogeously unidentifiability, unless the a priori restriction is made that the exchange rates of all proteins involved with the extravascular pools is identical, i.e. $\operatorname{TER}_{1 j}=\mathbb{T E R}_{1}, \operatorname{TER}_{2 j}=$ $\mathrm{TER}_{2}, \mathrm{ERR}_{1 j}=\mathrm{ERR}_{1}, \mathrm{ERR}_{2 j}=\mathrm{ERR}_{2}, j=1, \ldots, \mathrm{m}$.

It is however questionable whether such a refinement of the model, allowing a three exponential impulse response, is warranted for the plasma activity curves considered. For a slowly eliminated protein like albumin (FCR $=0.004 \mathrm{~h}^{-1}$ ) where the response upon a bolus injection of radioactivity labelled protein is monitored for more than a month one definetely may discern a triphasic disappearance curve. The plasma activity curves considered in this study however are observed for maximally fifteen days, are contaminated with an error of about 5\%, and concern proteins that are eliminated more than 3 times faster than albumin. As can be seen from the data presented in Table 3.2 the unit impulse response of an enzyme with $F C R=0.015 \mathrm{~h}^{-1}$ and with the other circulatory parameters derived from Mathews mammilary model for albumin [Beeken, 1962] can be approximated reasonably well by a bi-exponential curve. 
Table 3.2 Bi-exponential approximation of a triphasic unit inpulse response.

$\begin{array}{rccccc}\text { T } & C_{0} & \text { FCR } & \text { TER } & \text { E/P } & \text { Res } \\ 50 & 1.00 & 0.020 & 0.038 & 0.79 & 0.00033 \\ 100 & 1.00 & 0.018 & 0.038 & 0.92 & 0.00158 \\ 200 & 0.99 & 0.016 & 0.036 & 1.10 & 0.00390 \\ 400 & 0.98 & 0.015 & 0.036 & 1.22 & 0.00500\end{array}$

The tri-exponential unit impulse response function

$$
P_{b}(t)=0.495 e^{-0.1014 t}+0.228 e^{-0.0276 t}+0.277 e^{-0.0051 t}
$$

of Mathews Mammilary model with the circulatory parameters

$$
\begin{aligned}
\mathrm{FCR}=0.015 ; \mathrm{TER}_{1} & =0.013 ; \mathrm{E}_{1} / \mathrm{P}=1.06 \\
\mathrm{TER}_{2} & =0.030, \mathrm{E}_{2} / \mathrm{P}=0.50
\end{aligned}
$$

is approximated on the time interval $(0, T)$ by a least squares fit with the bi-exponential unit impulse response of the two-compartmental model. 


\subsection{The estimation procedure}

In order to apply the model (3.3) to patient data we must make the following adjustments: The response $c_{j}(t)$ effected by the transient input $f(t)$ generally is superimposed upon a non-zero basal steady state plasma level caused by aspecific enzyme release of various origins. Hence we have the expression

$$
c_{j}(t)=C s_{j}+(1 / \text { rhoj }) P_{b j}(t) * f(t)
$$

for the model plasma activity curve of enzyme $j$.

In contrast to the situation considered in the Appendix B.1 for the identifiability analysis where it was assumed that the response $C_{j}(t)$ was completely observed and free of error, the data collected in practice are sampled for a finite dicrete series of sample times $t_{1}, \ldots, t_{N}$ and are contaminated with an error $E_{i j}$.

The limited number of observations available necessitates the introduction of an approximation of the input function characte-

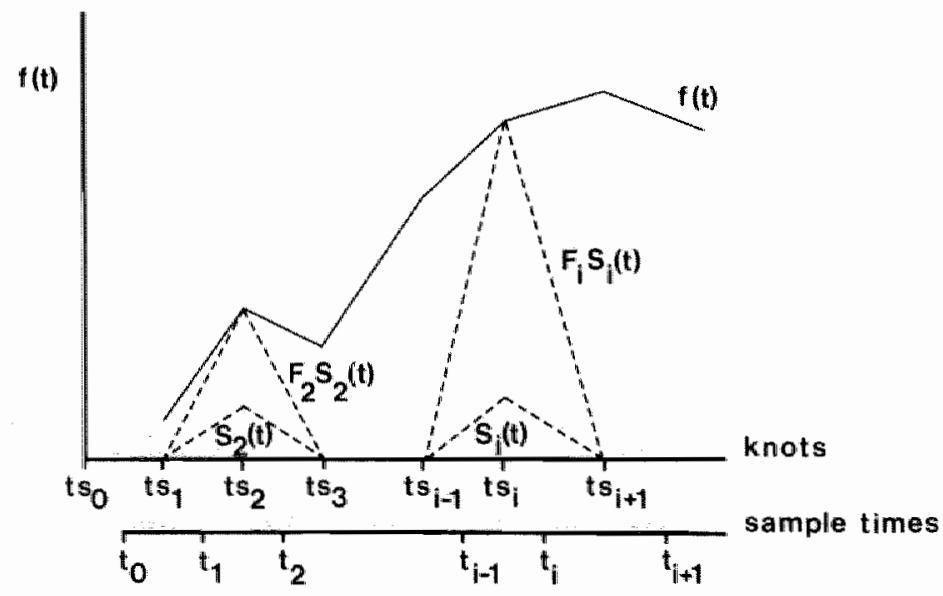

Fig. 3.4 The approximation of the input function $f(t)$ by a sum of "chapeau" funations $F_{i} S_{i}(t)$. The contributions $F_{2} S_{2}(t)$ and $F_{i} S_{i}(t)$ are thown in dotted lines. 
rised by a finite number of parameters. In this study it is choosen to approximate $f(t)$ by a sum of chapeau functions (1inear elements), ct. Fig. 3.4:

$$
f(t)=\sum_{i=1}^{N s} F_{i} S_{i}(t)
$$

defined on the knots $t_{s i}$ intermediate the sample times $t_{j}$ :

$$
\begin{aligned}
& t_{\text {so }}=-\frac{2}{2} t_{1} \\
& t_{s 1}=\frac{2}{2} t_{1} \\
& t_{\text {si }}=\frac{2}{2}\left(t_{i-1}+t_{i}\right)
\end{aligned}
$$

Clearly such a choice is to some extent arbitrary. There exist however some sound arguments for this choice. Firstly, this set of basis functions affords a flexible approximation also to functions $f(t)$ with a widely different behaviour in different ranges of the time. This in contrast to such obvious cholces as polynomials or exponential functions which often allow a more parsimonious approximation to smooth well behaved functions but that are too inflexible to follow abrupt changeover in the path of the function.

secondly, for such basis functions $S_{1}(t)$ it is possible to calculate explicitly (and efficiently) the convolution integral

$$
s_{i}(t) * e^{-k t}=\int_{0}^{t} e^{-k(t-\tau)} s_{i}(\tau) d t
$$

(cf. Appendix B.2), and thus of the modelfunction

$$
\begin{aligned}
C_{j}(t) & =C s_{j}+\left(1 / r h o_{j}\right)\left(P_{1 j} e^{-k 1 j t}+P_{2 j} e^{-k 2 j}\right) * f(t) \\
& =M(j, p, t)
\end{aligned}
$$

where all the parameters characterizing the model for the enzymes (cf. Formula (3.3)) are collected in the parameter vector p; $1 . e$. $\mathrm{p}=\left(\mathrm{CS}_{1}, \ldots, \mathrm{CS}_{\mathrm{m}}, \mathrm{rho} \mathrm{o}_{1}=1, \ldots, \mathrm{rho}_{\mathrm{m}}, \mathrm{FCR}_{1}, \ldots, \mathrm{ECR}_{\mathrm{m}}, \mathrm{TER}, \mathrm{ERR}, \mathrm{F}_{1}, \ldots, \mathrm{F}_{\mathrm{Ns}}\right)$ 
The estimation of these parameters from the measured data of the plasma activity curves now proceeds by a weighted (nonlinear) least squares fit: with the notation $c_{1 j}$ indicating the obsecved plasma activity of the j-th enzyme at the sample time $t_{1}, 1=1, \ldots, N$, and $r_{1 j}$ indicating the weighted residuals, $i . e$. the weighted deviation between the model function and the sampled data

$$
r_{i j}(p)=w_{i j}\left(C_{i j}-M\left(j, p, t_{i}\right)\right.
$$

with given weight factors $w_{i j}$ ' we define the sum of squared restaduals by

$$
\operatorname{SSR}(p)=\sum_{j=1}^{m} \sum_{i=1}^{\mathbb{N}} \mathrm{r}_{i j}{ }^{2}
$$

This function SSR(p) of the parameter values, given the observations $\mathrm{Cd} \mathrm{d}_{\mathrm{ij}}$, represents a measure for the deviation between the model with parameter values $p$ and the data. An obvious choice is then to estimate the parameter by minimizing the discrepancy ssR(p), i.e. choosing as estimate for $p$ the value of $p_{0}$ such that SSR(p) is minimal. This minimization is performed by using a modified Gauss-Newton procedure as described in Appendix B. 2 .

In the situation that the errors $\varepsilon_{i j}$ contaminating the observations of $C d_{i j}$ of $C_{j}\left(t_{i}\right)$ are independently distributed normal variates with mean zero and variance inversely proportional to $\left.1 / w_{i j}\right)^{2}, i \cdot e .:$

$$
w_{i j} E_{i j} \sim N\left(0, \sigma^{2}\right)
$$

This least squares estimate is a generally accepted estimator, (the Maximum Likelihood Estimator). For a further discussion of the properties of the estimator one is referred to the Appendix B. 3. 


\subsection{The performance of the estimation procedure}

The purpose of this section is to examine the small sample properties of the proposed estimator of the circulatory parameters.

\section{Generation_of_the_data_a_used_in_the_simulations}

In Fig. 3.5 the mean input function $f(t)$, as calculated for thirty patients with uncomplicated AMI, cf. [Willems, 1979] is shown together with the ad hoc approximation

$$
\begin{aligned}
f_{o}(t)=c t^{2} e^{-a t ; ~ w i t h ~} a & =0.111 \\
c & =0.78
\end{aligned}
$$

to this mean input function. This approximation $f_{o}(t)$ is used as a reference input function to calculate simulated data sets

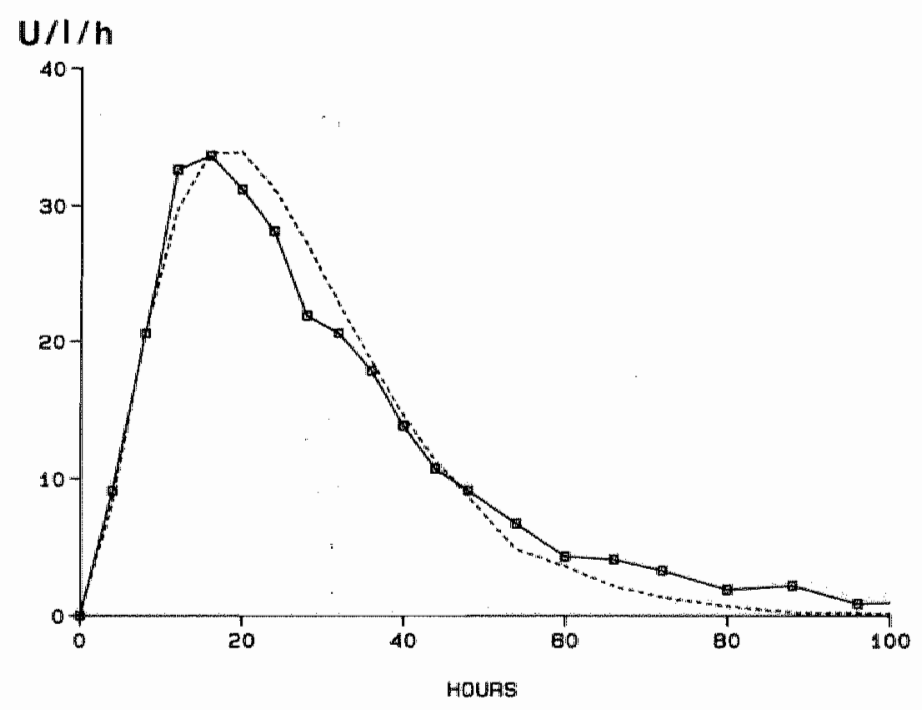

Fig. 3.5 The mean input of HBD into plasma calculated for 30 patienta with AMI uBing the values FCR $=0.015 \mathrm{~h}^{-1}, T E R=0.014 \mathrm{~h}^{-1}$ and $E R R=0.018 \mathrm{~h}^{-1}$ for the circulatory parameters (solid line). Also shown is the ad hoc approximation (3.8) (dotted line). 
conforming to the model (3.7) describing the plasma levels of simultaneously released enzymes. The plasma response upon this input equals the conwolution of $f_{0}(t)$ with the bi-exponential unit impulse response function, corresponding to the (given) reference values of the circulatory parameters (cf. Formula 3.3 ). In Appendix B.4 the formula for the explicit calculation of the resulting plasma activities is given. Unless specified otherwise the sample times of the simulated data are choosen conform the sample schedule usually practised with patient studies, i.e.

$\mathrm{T}=4,8,12, \ldots, 48,56,64,72,84,96,108,120,144,168, \ldots, 360$ (h)

cf. Chapter 4.2 .

As mentioned earliex in Appendix B.3, it is assumed that the sampling error is percentual and Gaussian. The sampling error is simulated by adding

$$
\varepsilon=\sigma C X
$$

with $X$ a Gaussian quasi random number with mean zero and variance 1 to the calculated plasma activity $C$. The size (standard deviation) of the error is determined by the scaling factor o. The quasi-random numbers $x$ are constructed by using the NAG library, subroutine G05DDF [NAGLIB].

Application_of the estimation_progedure to simulated_data

At first the case is consldered of two simultaneously released enzymes with values of the circulatory parameters corresponding to the cardiac enzymes $C K$ and $H B D$. In Table 3.3 the results obtained by fitting 100 simulated sets of plasma curves with various levels of error are summarized. Apparently even for the very small sampling error of 0.58 there results a (moderate) bias in the estimated value of the circulatory parameters presumably caused by the scarcity of the sampling schedule. Beyond this the results are satisfactory for sampling errors with o upto $2.5 \%$. For larger errors, i.e. with larger 0 , there occurs a conside- 
Table 3.3 Estimation of the circulatory parameters by simultaneous analysis of the plasma activity curves of two enzymes. Res \& TER ERR $\mathrm{CS}_{1} \mathrm{rho}_{1} \underset{\begin{array}{c}\mathrm{rho}_{1} \mathrm{CS}_{2} \\ \mathrm{FCR}_{1}\end{array}}{\mathrm{rho}_{2}} \mathrm{FCR}_{2} \begin{aligned} & \mathrm{rho}_{2} \\ & \mathrm{FCR}_{2}\end{aligned}$

\begin{tabular}{|c|c|c|c|c|c|c|c|c|c|c|c|}
\hline \multicolumn{2}{|c|}{$\begin{array}{c}\text { Reference } \\
\text { values }\end{array}$} & -- & 0.0140 & 0.0180 & 80.0 & 1.0 & 0.0150 & 45.0 & 0.140 & 0.200 & 0.028 \\
\hline \multirow{2}{*}{\multicolumn{2}{|c|}{$\mathrm{O}=0.5{ }^{\text {mean }}$}} & 0.7 & $0.015 i$ & 0.0191 & 80.4 & 1.0 & 0.0153 & 45.7 & 0.136 & 0.240 & 0.02 \\
\hline & & 0.1 & 0.0004 & 0.0003 & 0.5 & - & 0.0002 & 0.1 & 0.003 & 0.006 & 0.00 \\
\hline \multirow{2}{*}{$0=0.58$} & $\delta$ & - & 0.0006 & 0.0012 & 1.5 & - & 0.0006 & - & 0.008 & 0.018 & 0.00 \\
\hline & mean & 1.1 & 0.0151 & 0.0191 & 80.4 & 1.0 & 0.0153 & 45.7 & 0.136 & 0.210 & 0.0 \\
\hline \multirow[t]{3}{*}{$\sigma=1.08$} & $S D$ & 0.1 & 0.0009 & 0.0006 & 0.9 & - & 0.0004 & 0.1 & 0.005 & 0.011 & 0.00 \\
\hline & $\delta$ & - & 0.0024 & 0.0019 & 2.3 & - & 0.009 & - & 0.012 & 0.028 & 0. \\
\hline & mean & 2.5 & 0.0152 & 0.0191 & 80.2 & 1.0 & 0.0153 & 45.7 & 0.137 & 0.210 & \\
\hline \multirow[t]{3}{*}{$0=2.58$} & $\mathrm{SD}$ & 0.3 & 0.0023 & 0.0016 & 2.3 & - & 0.0009 & 0.3 & 0.013 & 0.029 & 0.00 \\
\hline & $\delta$ & - & 0.0055 & 0.0043 & 5.2 & - & 0.0021 & - & 0.026 & 0.063 & 0.0 \\
\hline & mean & 4.9 & 0.0158 & 192 & 80.0 & 1.0 & & 45.7 & & 24 & \\
\hline \multirow[t]{3}{*}{$a=5.0 \%$} & $S D$ & 0.7 & 0.0054 & 0.0033 & 4.6 & - & 0.0020 & 0.6 & 0.026 & 0.084 & 0. \\
\hline & $\delta$ & - & 0.0117 & 0.0086 & 10.2 & - & 0.0043 & - & 0.053 & 0.156 & 0.0 \\
\hline & mean & 7.5 & 0.0166 & 0.0195 & 81.5 & 1.0 & 0.0157 & 45.7 & 0.137 & 0.239 & 0.0 \\
\hline \multirow[t]{2}{*}{$\sigma=7.58$} & SD & 0.9 & 0.0079 & 0.0051 & 7.4 & - & 0.0029 & 0.9 & 0.038 & 0.118 & 0.0 \\
\hline & $\delta$ & - & 0.0194 & 0.0136 & 15.5 & - & 0.0069 & - & 0.081 & 0.288 & 0.0 \\
\hline
\end{tabular}

Indlcated figures are obtained mean and standard deviation as obtained from 100 independently simulated data sets. Plasma activity curves were constructed using the indicated reference values for rho and the circulatory parameters and the reference input function $f_{0}(t)$ Erom Formula (3.8); calculations are given more explicitly in Appendix B.4. For each estimated parameter value $\mathrm{p}$ also an approximate 958-confidence interval $(\mathrm{p}-\delta, \mathrm{p}+\delta)$ was calculated. The presented figures are the mean values as obtained in 100 gifnulations, indicating the (half of) the size of this confidence interval.

The simulated data were contaminated with independent percentual sampling error with a standard deviation of 08 . This gampling error was generated by a quasi-random generator slmulating a Gaussian distributed random variable. The value of $\mathrm{Cs}_{2}$ of the rapidly eliminated enzyme (s) is independently estimated as the mean of the data after 192 hours.

Res - is the estimated value of the standard deviation of the percentual erxor expressed in percents. 

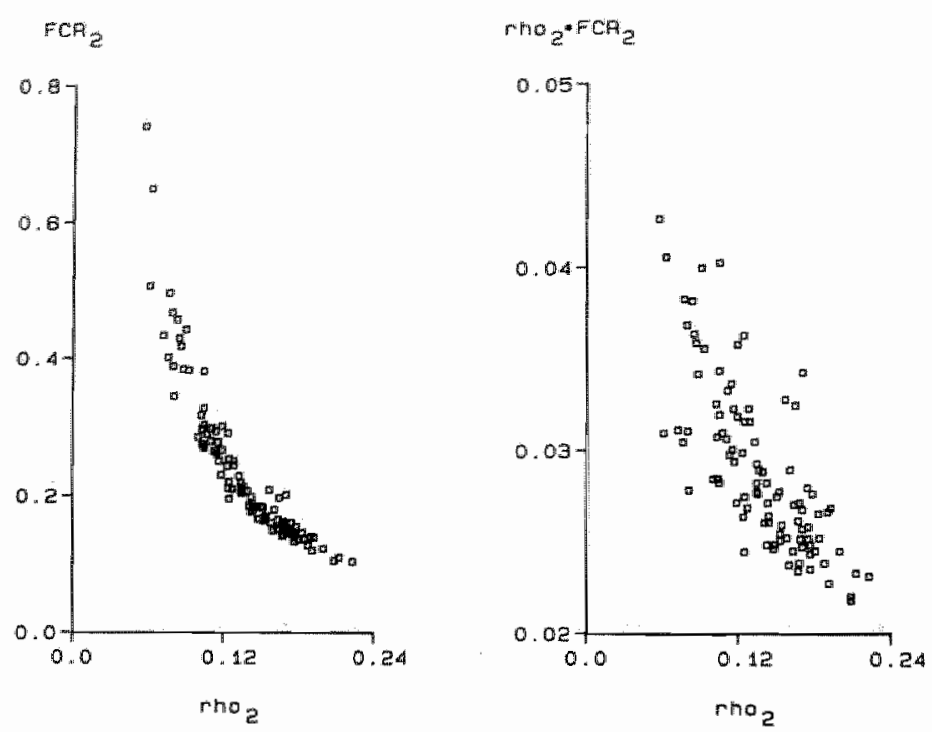

Fig. 3.6 The estimated values of $\mathrm{FCR}_{2}$ and of rho $\mathrm{H}_{2}$ obtained from simulated plasma activity curves contaminated with $7.5 \%$ error. Left panel the corretation between ${ }^{2 h O_{2}}$ and $\mathrm{ECR}_{2}$; Right panel the correlation between $\mathrm{rho}_{2}$ and $\mathrm{rho}_{2} \mathrm{FCR}_{2}$.

rable bias in the estimation of $\mathrm{FCR}_{2}$. From the plot, Fig. 3.6, of the individual values of the estimated rho and $\mathrm{FCR}_{2}$ for $\sigma=7.5$ \% it appears that this overestimation of $\mathrm{FCR}_{2}$ is caused by a few outlying values while also a high correlation between rho and $\mathrm{FCR}_{2}$ is apparent. In Fig. 3.7 of the residual contours (for unperturbed data) as a function of rho and $\mathrm{FCR}_{2}$ exhibits a pronounced deviation from quadratic dependence of SSR (the sum of the squared cesiduals) on the parameter values rho and $\mathrm{FCR}_{2}$. Apparently the outliers and the bias are caused by the non-linearity of the regression problem, which can be remedied by the transformation of the variables. In Fig. 3.7 of the residual contours (for unperturbed data) as a function of $\mathrm{rho}_{2}$ and $\mathrm{FCR}_{2}$ exhibits a pronounced deviation from quadratic dependence of SSR (the sum of the squared residuals) on the parameter values rho and FCR. Apparently the outliers and the bias are caused by the non-linearity of the regression problem, which can be remedied by the transformation of the variables (rho, FCR) to the variables (rho, rho.FCR), cf. Fig. 3.7. 

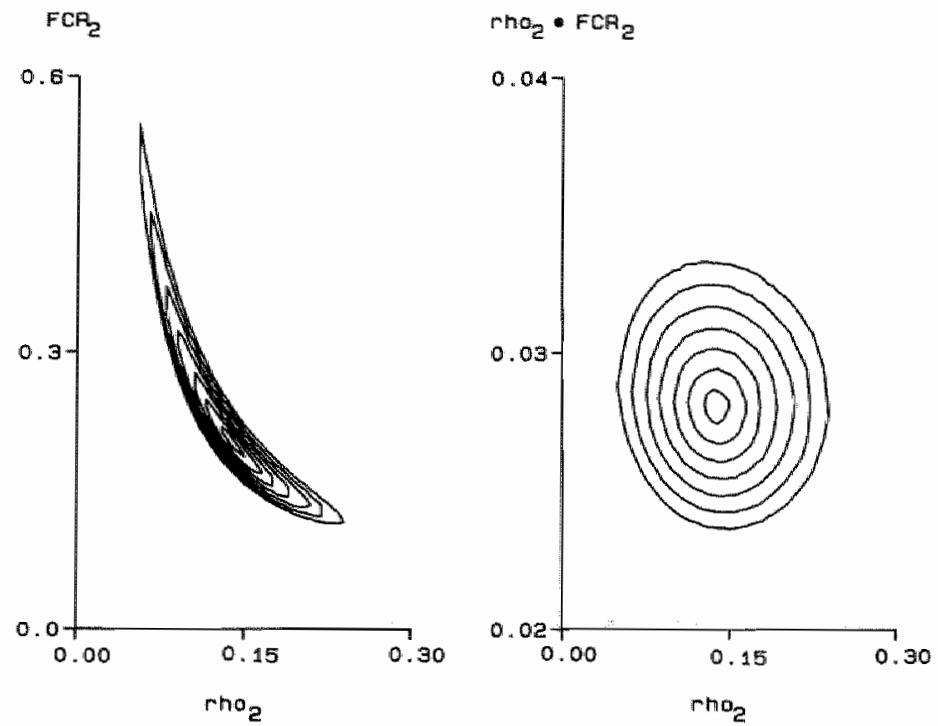

Fig. 3.7 The contours of the mean deviation between modet function and unperturbed simulated data comesponding to the meference value of Table 3.3. Indicated are the Levels Reas $=0.01, \ldots, 0.07$. The modet function is fitted to unperturbed data with respeat of the nutssance parametere $\mathbb{F}_{1}, \ldots, F_{N S}$, while the circulatory modet paraneterg are kept fixed. The obtained Rea\% is a function of rho and FCR 2 *

Left panet: the contours of Ress a a function of rho and $F_{2}$. Right panel: the contours of Res\% as a function of rho and mho $_{2} \cdot \mathrm{FCR}_{2}$ 
The data in Table 3.3. also indicate that the estimation of $\mathrm{rho}_{2} \cdot \mathrm{FCR}_{2}$ is unbiased in contrast to the estimation of $\mathbb{E C R}$. Thus we consider henceforth the estimation problem ith the parameters CS, rho, rho. PCR, TER and ERR.

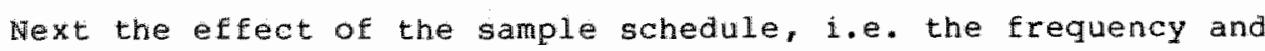
the duration of the sampling, upon the variation in the estimates is studied: Hereto simulated data are constructed, according to the procedure described above, for several modifications of the original siample schedule Ts.

From the results presented in Table 3.4 it is apparent that estimation of TER and ERF with a reasonible accuracy reguires sampling at least up to 240 hours, while longer collection of samples thereater hardly contributes to improved accuracy. A further conclusion is that improved results may be attained by a more frequent sample collection during the first 24 hours. Lastly it appears that increasing the number of samples after 120 hours does not lead to a improved estimation.

A further question regards dependence of the estimation procedure upon the values of the circulatory parameters, especially upon the values of FCR, of the enzymes included in the analysis. In Table 3.5 the results of the estimation of the circulatory model parameters from simulated plasma activity curves for various combinations of the reference values of the $\mathrm{FCR}_{1}$ and $\mathrm{FCR}_{2}$ are given. From these data it appears that:

- For an adequate determination of the circulatory model parameters it is indispensable that one of the enzymes included in the analysis is catabolised at a very slow rate. Even an increase of FCR $=0.015 \mathrm{~h}^{-1}$ to the value FCR $=0.05 \mathrm{~h}^{-1}$ results in a considerable increase of the variance of the estimated parameter values.

- The performance of the estimation procedure improves with greater difference in the values of FCR of the two enzymes analysed and the results deteriorate for values of FCR in the same range, e.g. the combination $\mathrm{FCR}_{1}=0.015 \mathrm{~h}^{-1}$ and $\mathrm{FCR}_{2}=0.050 \mathrm{~h}^{-1}$ gives disputable results. 
Table 3.4 Estimation of the circulatory paxameters from simulated plasma activity curves contaminated with 5 random error: Effect of the sample schedules.

Res TER ERR CS, tho $\begin{array}{llll}\mathrm{ThO}_{1} & \mathrm{CS}_{2} & \text { tho } & \mathrm{rho}_{2} \\ \mathrm{FCR}_{1} & & \mathrm{FCR}_{2}\end{array}$

Reference $\quad-0.0140 \quad 0.018080 .0 \quad 1.0 \quad 0.015045 .0 \quad 0.140 \quad 0.0280$ values

Ts, In this case no thrustworthy results could be obtained due to lack of convergence.

\begin{tabular}{|c|c|c|c|c|c|c|c|c|c|c|}
\hline $\mathbf{s}_{2}$ & $\begin{array}{c}\text { mean } \\
\mathrm{SD}\end{array}$ & $\begin{array}{l}5.1 \\
1.1\end{array}$ & $\begin{array}{l}0.0207 \\
0.0113\end{array}$ & $\begin{array}{l}0.0204 \\
0.0168\end{array}$ & $\begin{array}{r}79.7 \\
4.8\end{array}$ & $\begin{array}{r}1.0 \\
-\end{array}$ & $\begin{array}{l}0.0140 \\
0.0057\end{array}$ & $\begin{array}{r}45.7 \\
0.6\end{array}$ & $\begin{array}{l}0.138 \\
0.030\end{array}$ & $\begin{array}{l}0.0289 \\
0.0054\end{array}$ \\
\hline & $\begin{array}{c}\text { mean } \\
\mathrm{SD}\end{array}$ & $\begin{array}{l}4.9 \\
0.8\end{array}$ & $\begin{array}{l}0.01157 \\
0.0058\end{array}$ & $\begin{array}{l}0.0187 \\
0.0060\end{array}$ & $\begin{array}{r}79.6 \\
4.7\end{array}$ & 1.0 & $\begin{array}{l}0.0151 \\
0.0022\end{array}$ & $\begin{array}{r}45.7 \\
0.6\end{array}$ & $\begin{array}{l}0.140 \\
0.027\end{array}$ & $\begin{array}{l}0.0283 \\
0.0030\end{array}$ \\
\hline & $\begin{array}{c}\text { mean } \\
\text { SD }\end{array}$ & $\begin{array}{l}5.0 \\
0.7\end{array}$ & $\begin{array}{l}0.0159 \\
0.0056\end{array}$ & $\begin{array}{l}0.0193 \\
0.0042\end{array}$ & $\begin{array}{r}79.8 \\
4.6\end{array}$ & $\begin{array}{c}1.0 \\
-\end{array}$ & $\begin{array}{l}0.0154 \\
0.0021\end{array}$ & $\begin{array}{r}45.7 \\
0.6\end{array}$ & $\begin{array}{l}0.138 \\
0.026\end{array}$ & $\begin{array}{l}0.0286 \\
0.0032\end{array}$ \\
\hline & $\begin{array}{c}\text { mean } \\
\mathrm{SD}\end{array}$ & $\begin{array}{l}4.9 \\
0.7\end{array}$ & $\begin{array}{l}0.0158 \\
0.0054\end{array}$ & $\begin{array}{l}0.0192 \\
0.0033\end{array}$ & $\begin{array}{r}80.0 \\
4.6\end{array}$ & $\begin{array}{c}1.0 \\
-\end{array}$ & $\begin{array}{l}0.0155 \\
0.0020\end{array}$ & $\begin{array}{r}45.7 \\
0.6\end{array}$ & $\begin{array}{l}0.137 \\
0.026\end{array}$ & $\begin{array}{l}0.0286 \\
0.0032\end{array}$ \\
\hline & $\begin{array}{c}\text { mean } \\
\mathrm{SD}\end{array}$ & $\begin{array}{l}4.9 \\
0.7\end{array}$ & $\begin{array}{l}0.0158 \\
0.0054\end{array}$ & $\begin{array}{l}0.0191 \\
0.0032\end{array}$ & $\begin{array}{r}90.1 \\
3.6\end{array}$ & $\begin{array}{c}1.0 \\
-\end{array}$ & $\begin{array}{l}0.0155 \\
0.0020\end{array}$ & $\begin{array}{r}45.7 \\
0.6\end{array}$ & $\begin{array}{l}0.137 \\
0.02 .4\end{array}$ & $\begin{array}{l}0.0287 \\
0.0032\end{array}$ \\
\hline G & $\begin{array}{c}\text { mean } \\
\text { SD }\end{array}$ & $\begin{array}{l}4.9 \\
0.5\end{array}$ & $\begin{array}{l}0.0162 \\
0.0052\end{array}$ & $\begin{array}{l}0.0196 \\
0.0036\end{array}$ & $\begin{array}{r}80.1 \\
3.5\end{array}$ & $\begin{array}{c}1.0 \\
-\end{array}$ & $\begin{array}{l}0.0157 \\
0.0019\end{array}$ & $\begin{array}{r}45.7 \\
0.6\end{array}$ & $\begin{array}{l}0.134 \\
0.021\end{array}$ & $\begin{array}{l}0.0289 \\
0.0031\end{array}$ \\
\hline & $\begin{array}{c}\text { mean } \\
\text { SD }\end{array}$ & $\begin{array}{l}4.9 \\
0.5\end{array}$ & $\begin{array}{l}0.0154 \\
0.0045\end{array}$ & $\begin{array}{l}0.0201 \\
0.0035\end{array}$ & $\begin{array}{r}79.8 \\
2.0\end{array}$ & $\begin{array}{c}1.0 \\
-\end{array}$ & $\begin{array}{l}0.0152 \\
0.0015\end{array}$ & $\begin{array}{r}45.7 \\
0.6\end{array}$ & $\begin{array}{l}0.137 \\
0.016\end{array}$ & $\begin{array}{l}0.0285 \\
0.0027\end{array}$ \\
\hline & $\begin{array}{c}\text { mean } \\
\text { SD }\end{array}$ & 1.9 & $\begin{array}{l}0.0169 \\
0.0057\end{array}$ & $\begin{array}{l}0.0201 \\
0.0034\end{array}$ & $\begin{array}{r}82.0 \\
4.8\end{array}$ & 1.0 & $\begin{array}{l}0.0161 \\
0.0021\end{array}$ & $\begin{array}{r}45.7 \\
0.6\end{array}$ & $\begin{array}{l}0.128 \\
0.022\end{array}$ & $\begin{array}{l}0.02 \\
0.00\end{array}$ \\
\hline
\end{tabular}

Cf. Table 3.3.

$T s_{o}: T=4,8,12, \ldots, 48,56,64,72,84,96,108,120,144, \ldots, 360(\mathrm{~h})$

$T s_{1}$ - the sample sichedule $T s_{0}$ restricted to 120 hoursi

$\mathrm{TIS}_{2}$ - the sample schedule $\mathrm{Ts}_{\mathrm{o}}$ restricted to 180 hours

$\mathrm{Ts}_{3}$ - the sample schedule $\mathrm{Ts}$ o restricted to 240 hours

$\mathrm{Ts}_{4}$ - the sample schedule $\mathrm{Ts}$ restricted to 300 hours

$\mathrm{Ts}_{5}$ - the sample schedule $\mathrm{Ts}_{0}^{0}$ extended upto 480 hours by daily samples

$T s_{6}-T s_{0}$ extended with two-hourly samples upto 24 hours

$\mathrm{Ts}_{7}-\mathrm{Ts}_{6}^{0}$ extended with hourly samples upto 12 hours

Ts $s_{8}-$ Ts $_{0}$ extended with 12 -hourly samples between $120-240$ hours 
Table 3.5 Estimation of the cixculatory parameters from simulated plasma activity curves contaminated with $5 \%$ error: Effect of the values of FCR.

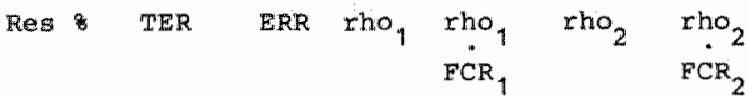

Reference values

Estimated; mean

SD

Reference values

Estimated: mean

SD

Reference values

Estimated: mean

SD

Reference values

Estimated: mean SD

Reference values

Estimated: mean SD

Reference values

Estimated: mean SD

Reference values Estimated: mean $\mathrm{SD}$

Reference values Estimated: mean SD

Reference values Estimated" mean $\mathrm{SD}$

$$
\begin{array}{ccc}
- & 0.0140 & 0.0180 \\
5.0 & 0.0241 & 0.0186 \\
0.8 & 0.0288 & 0.0072
\end{array}
$$

1.00 .0150

$1.0 \quad 0.0191$

$-0.0125$

$-\quad 0.01400 .0180$

1.00 .0150

$\begin{array}{lllll}5.0 & 0.0180 & 0.0212 & 1.0 & 0.0166\end{array}$

$0.6 \quad 0.0077 \quad 0.0034$

$-0.0032$

$-\quad 0.0140 \quad 0.0180$

1.00 .0150

$\begin{array}{llllll}4.9 & 0.0158 & 0.0992 & 1.0 & 0.0155\end{array}$

$0.7 \quad 0.0064 \quad 0.0033$

$-0.0020$

$\begin{array}{lllll}- & 0.0140 & 0.0180 & 1.0 & 0.0150\end{array}$

$\begin{array}{llllll}5.0 & 0.0157 & 0.0184 & 1.0 & 0.0152\end{array}$

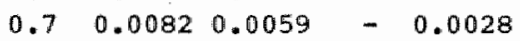

$-\quad 0.01400 .019020 .0 \quad 0.050$
-0.0264

$\begin{array}{llllll}5.0 & 0.0264 & 0.0270 & 20.0 & 0.072\end{array}$

$0.70 .02550 .0159-0.039$

$-\quad 0.0140 \quad 0.018020 .0 \quad 0.060$

$\begin{array}{llllll}5.0 & 0.0181 & 0.0242 & 20.0 & 0.066\end{array}$

$0.70 .01160 .0101-0.012$

$$
\begin{aligned}
& -\quad 0.0140 \quad 0.018020 .0 \quad 0.050
\end{aligned}
$$

$\begin{array}{llllll}4.9 & 0.0244 & 0.0267 & 20.0 & 0.056\end{array}$

$0.7 \quad 0.0236 \quad 0.216$

$-0.021$

$\begin{array}{r}-\quad 0.0140 \quad 0.0180 \\ \hline .001920 .0253\end{array}$

$\begin{array}{lll}5.0 & 0.0192 & 0.0253\end{array}$

$\begin{array}{llll}0.7 & 0.0237 & 0.0197\end{array}$

2.40 .100

2.40 .117

$-0.035$

$\begin{array}{ccccc}- & 0.0140 & 0.0186 & 2.4 & 0.100 \\ 4.9 & 0.0299 & 0.0343 & 2.4 & 0.128 \\ 0.7 & 0.0318 & 0.0246 & - & 0.052\end{array}$ $\begin{array}{rr}20.0 & 1.00 \\ 19.3 & 1.25 \\ 3.1 & 0.70\end{array}$

$2.40 \quad 0.240$

$2.29 \quad 0.261$

$0.34 \quad 0.044$

$\begin{array}{lll}0.140 & 0.0280\end{array}$

$\begin{array}{lll}0.137 & 0.0288\end{array}$

$0.026 \quad 0.0033$

$$
\begin{array}{ll}
1.00 & 0.300 \\
1.02 & 0.307 \\
0.34 & 0.047
\end{array}
$$

$2.40 \quad 0.240$

$2.19 \quad 0.323$

$0.47 \quad 0.177$

$0.140 \quad 0.0280$

0.1310 .0312

$0.030 \quad 0.0083$

$1.00 \quad 0.300$

$0.93 \quad 0.344$

$0.40 \quad 0.127$

$0.140 \quad 0.0280$

$\begin{array}{lll}0.132 & 0.0329\end{array}$

$0.023 \quad 0.0106$

$1.00 \quad 0.300$

$0.87 \quad 0.384$

$0.29 \quad 0.154$

Cf. Table 3.3

In these simulations the following prototypes of the cardiac enzymes are studied:

$\begin{array}{llll}\mathrm{HBD} & \text { : rho }=1.0, & \mathrm{FCR}=0.015 ; \text { rho. } \mathrm{FCR}=0.015 \\ \mathrm{ALT} & \text { : rho }=20.0, & \mathrm{FCR}=0.050 ; \text { rho.FCR }=1.00 \\ \mathrm{AST} & \text { : rho }=2.4, & \mathrm{FCR}=0.10 ; \text { rho.FCR }=0.24 \\ \mathrm{CK} & \text { : rho }=0.14, \mathrm{FCR}=0.20 ; \text { rho.FCR }=0.028 \\ \mathrm{CK}-\mathrm{MB} / \mathrm{GPI} & \text { : rho }=1.0, \mathrm{FCR}=0.30 ; \text { rho.FCR }=0.30\end{array}$


Table 3.6 Estimation of the circulatory parameters from simulated plasma activity curves contamimated with 58 random error: The method applied to the amalysis of three simultaneously sampled plasma activity curves.

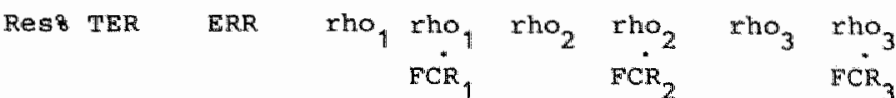

\begin{tabular}{|c|c|c|c|c|c|c|c|c|c|c|}
\hline eference & values & - & 0.0140 & 0.0180 & 1.0 & 0.0150 & 0.140 & 0.0280 & 20.0 & 1.00 \\
\hline stimated: & me & 4.9 & 0.0153 & 0.0190 & 1.0 & 0.0153 & 0.138 & 0.0285 & 19.9 & 1.02 \\
\hline & SD & 0. & 0.0044 & 0.0030 & - & 0.0018 & 0.023 & 0.0032 & 1.1 & 0.11 \\
\hline af & & - & & & 1.0 & & & & 2.4 & \\
\hline+ & mea & 5.0 & 0. & 0. & 1.0 & 0. & 0.133 & 0 . & 2.32 & 0 \\
\hline & SD & 0.5 & 0.0 & 0.0 & - & 0. & 0.025 & 32 & 0.21 & 0.02 \\
\hline efer & & - & 10 & & 1.0 & 0 & 40 & 0 . & 1. & 30 \\
\hline 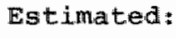 & & 5.0 & 0.0158 & $0.0 \| 94$ & 1.0 & 0.0154 & 0.140 & 0.0287 & 0.98 & 30 \\
\hline & SD & 0.5 & 0.0048 & 0.0034 & - & 0.0018 & 0.024 & 0.0029 & 0.25 & 0.030 \\
\hline
\end{tabular}

Fit of the data of enzymes 1 and 2 up to 360 hours, while the data of enzyme 3 are fitted only up to 48 hours

\begin{tabular}{|c|c|c|c|c|c|c|c|c|c|c|}
\hline eference & lues & - & 0 & .0180 & 1.0 & 0 . & 0.140 & 0.0280 & 20.0 & 1.90 \\
\hline stimated: & mean & 4.9 & 0.0158 & 0.0192 & 1.0 & 0.0154 & 0.138 & 0.0287 & 20.11 & 1.02 \\
\hline & SD & 0.6 & 0. & 033 & - & 019 & 0.026 & 0.0031 & 2.0 & 0.16 \\
\hline & val & - & 10 & & 1.0 & & 0. & 80 & 2.40 & \\
\hline & mer & 5.0 & 0.0 & 0. & 1.0 & 0. & 0.136 & 89 & 2.39 & 2 \\
\hline & SD & 0.6 & 0. & 33 & - & 0. & 0.026 & 032 & 0.27 & 0.03 \\
\hline$x^{2}$ & & - & & & 1.0 & & 0.140 & & 1.00 & \\
\hline 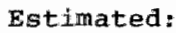 & & 4.9 & 0.0 & 0.0193 & 1.0 & 0.0 & 0.138 & 0.0288 & 0.98 & $a r$ \\
\hline & & 0.6 & 0.0051 & 0.0034 & - & 0.0019 & 0.025 & 0.0029 & 0.26 & .0 \\
\hline
\end{tabular}

Cf. Table 3.3

These findings stress the necessity of a multi-enzyme analysis for the determination of the value FCR of enzymes eliminated at a rate between $\operatorname{HBD}\left(\mathrm{FCR}=0.015 \mathrm{~h}^{-1}\right)$ and $\operatorname{AST}\left(\mathrm{ECR}=0.093 \mathrm{~h}^{-1}\right)$.

In Table 3.6 results obtained by simultaneous analysis of 3 simulated plasma activity curves with 5 error are given. Also are shown results obtained by fitting the model to complete data upto 360 hours of two of the three enzymes while from one of the enzymes only data upto 48 hours are used.

From these data it is concluded that for enzymes with intermediate values of $\mathrm{FCR}$, say $0.02<\mathrm{FCR} 0.10$ such an analysis results 
in an improved detemination of the value of FCR. Another conclusion is that if one expects release of some enzyme from other tissues (which would invalidate the model (3.7)) the estimation of FCR still is feasible by a multi-enzyme analysis and by limitation of the time interval for these enzymes. 


\subsection{Departures from the model}

In the previous section it was demonstrated that the least squares regression allows a satisfactory estimation of the circulatory parameters when the following assumptions are valid:

(A1) The dynamics of the plasma levels of the simultaneously analysed proteins can be described by the simplified two compartmental model (2.4) with identical valus for TER and ERR for all proteins.

(A2) The release of the enzymes is described by the same input function $f(t), i . e$. the input $f_{j}(t)$ of enzyme $j$ is given by

$$
f_{j}(t)=\left(1 / r h o_{j}\right) f(t)
$$

As mentioned in Appendix B.3 the detection of such departures from the model by residual analysis is hampered by the presence of the large number of (nuissance) parameters in the model causing highly correlated residuals and invalidating the common tests for the detection of systematic deviations. Therefore the alternative is choosen to analyse the effects of such departures on the estimation by simulations.

\section{Deviating_cirgculatatory_models}

In Table 3.7 we present the estimated values of the two-compartment circulatory parameters as obtained by regression of the model (3.7) on error free data generated according to Appendix B. 4 with the three exponential unit impulse response for the Mathews three-compartment mammilary model corresponding to the indicated reference values of the circulatory parameters (instead of the bi-exponential unit impulse response of the simplified two-compartment model).

Apparently the application of the two-compartment model to such data conforming the deviant three compartment model does not affect the estimated values of $\mathrm{rho}_{2}, \mathrm{FCR}_{1}$ and $\mathrm{FCR}_{2}$ to a large 
Table 3.7 Estimation of the circulatory parameters of the twocompartmental model from data calculated for the threecompartmental mamial 1 ary node1.

$\mathrm{TER}_{1} \mathrm{E}_{1} / \mathrm{p} \quad \mathrm{TER}_{2} \mathrm{E}_{2} / \mathrm{p} \quad \mathrm{FCR}_{1} \mathrm{ThO}_{2} \mathrm{FCR}_{2}$ Rest

\begin{tabular}{|c|c|c|c|c|c|c|c|c|c|}
\hline Reterence & Waluas & 0.030 & 0.0 .5 & 0.015 & 0.95 & 0.015 & 0.14 & 0.20 & - \\
\hline E.it & & 0.016 & 0.92 & - & - & 0.015 & 0.13 & 0.21 & 0.8 \\
\hline Reference & values & 0.030 & 0.10 & 0.015 & 0.90 & 0.015 & 0.14 & 0.20 & - \\
\hline it & & 0.016 & 0.85 & - & - & 0.014 & 0.14 & 0.19 & 0.7 \\
\hline Reference & values & 0.030 & 0.20 & 0.015 & 0.80 & 0.015 & 0.1 .4 & 0.20 & - \\
\hline it & & 0.016 & 0.72 & - & - & 0.013 & 0.15 & 0.17 & 0.6 \\
\hline Reference & values & 0.030 & 0.40 & 0.015 & 0.50 & 0.015 & 0.14 & 0.20 & - \\
\hline$E^{1} \mathrm{t}$ & & 0.024 & 0.74 & - & - & 0.013 & 0.16 & 0.16 & 0.4 \\
\hline Reference & values & 0.030 & 0.60 & 0.0115 & 0.40 & 0.015 & 0.14 & 0.20 & - \\
\hline Fit & & 0.043 & 0.99 & - & - & 0.015 & 0.14 & 0.20 & 0.1 \\
\hline Reference & values & 0.030 & 0.80 & 0.015 & 0.20 & 0.015 & 0.14 & 0.20 & - \\
\hline FIt & & 0.038 & 0.93 & - & - & 0.014 & 0.14 & 0.19 & 0.2 \\
\hline Reference & values & 0.030 & 0.90 & 0.015 & 0.10 & 0.015 & 0.14 & 0.20 & - \\
\hline$F \perp t$ & & 0.031 & 0.88 & - & - & 0.014 & 0.14 & 0.18 & 0.2 \\
\hline Reference & values & 0.030 & 0.95 & 0.015 & 0.05 & 0.015 & 0.14 & 0.20 & - \\
\hline it & & 0.030 & 0.93 & - & - & 0.015 & 0.14 & 0.19 & 0.3 \\
\hline
\end{tabular}

Data sets for both enzymes were generated by convolution of the three-exponential unit impulse response functions of Mathews mamnilary model corresponding to the indicated reference values of the citculatory parameters with the reference input function $(3.13)$

Indilited estimatong are obtained by fitting these unperturbed data with the two-compartmental model $(3.7)$.

extent while the estimated values of $\mathrm{E} / \mathrm{P}=\mathrm{TER} / \mathrm{ERR}$ represent a reasonible good approximation to the total extravascular pool, $\mathrm{E} / \mathrm{P}=\mathrm{E}_{1} / \mathrm{P}+\mathrm{E}_{2} / \mathrm{P}$

A further assumption regards the idential exchange rates between the plasma pool and the extravascular pool for all enzymes analysed simultaneously. The effect of departures from this assumption on the estimation are shown in Table 3.8. The data presented in this table indicate that the values of TER estimated 
Table 3.8 The effect of differences in the exchange rates between plasma and the extravascular pool for the various enzymes:

\begin{tabular}{|c|c|c|c|c|c|c|c|c|}
\hline fere & ce values & $\begin{array}{c}\text { TER } \\
0.015\end{array}$ & $\begin{array}{l}\mathrm{ERR}_{1} \\
0.015\end{array}$ & $\begin{array}{l}\text { rho, } \\
1.0\end{array}$ & $\begin{array}{l}\mathrm{FCR}_{1} \\
0.015\end{array}$ & $\begin{array}{r}\text { tho } \\
0.14\end{array}$ & $\begin{array}{r}F C R_{2} \\
0.21\end{array}$ & Res: \\
\hline $\mathrm{TER} \mathrm{R}_{\mathrm{2}}$ & $\mathrm{ERR}_{2}$ & & & & & & & \\
\hline 0.010 & 0.0067 & 0.016 & 0.017 & 1.0 & 0.015 & 0.14 & 0.21 & 0.6 \\
\hline 0.010 & 0.0 ม 00 & 0.016 & 0.017 & 1.0 & 0.015 & 0.14 & 0.21 & 0.7 \\
\hline 0.010 & 0.0150 & 0.017 & 0.017 & 1.0 & 0.016 & 0.13 & 0.22 & 0.9 \\
\hline 0.015 & 0.0100 & 0.015 & 0.016 & 1.0 & 0.015 & 0.13 & 0.24 & 0.7 \\
\hline 0.015 & 0.0150 & 0.017 & 0.016 & 1.0 & 0.016 & 0.14 & 0.22 & 0.9 \\
\hline 0.015 & 0.0225 & 0.019 & 0.018 & 1.0 & 0.016 & 0.13 & 0.23 & 4.2 \\
\hline 0.020 & 0.0133 & 0.016 & 0.015 & 1.0 & 0.016 & 0.14 & 0.21 & 0.8 \\
\hline 0.020 & 0.0200 & 0.018 & 0.017 & 1.0 & 0.017 & 0.13 & 0.24 & 1.0 \\
\hline 0.020 & 0.0300 & 0.021 & 0.018 & 1.0 & 0.017 & 0.12 & 0.25 & 1.3 \\
\hline 0.030 & 0.0200 & 0.018 & 0.016 & 1.0 & 0.017 & 0.13 & 0.24 & 1.0 \\
\hline 0.030 & 0.0300 & 0.022 & 0.017 & 1.0 & 0.018 & 0.12 & 0.27 & 1.3 \\
\hline 0.030 & 0.0450 & 0.024 & 0.019 & 1.0 & 0.018 & 0.12 & 0.27 & 1.4 \\
\hline
\end{tabular}

Simulated data are calculated according to Appendix B.4 with given refexence values of the circulatory parameters. Reference values of $\mathrm{TER}_{2}$ and $\mathrm{ERR}_{2}$ are varied as indicated in the lefthand columnis. Presented values are obtained by regression of the model (3.7) to unperturbed data sets.

according to the model (3.7) are a compromise between the values of TER of both enzymes, while the estimates of the other circulatory parameters may show a bias of upto $25 \%$.

Another issue concerns the applicability of the one-compartment model for the simultaneous analysis of plasma enzyme activity curves, as is suggested by the nearly mono-exponential unit impulse response curves of rapidy eliminated proteins, cf. Table 3.1. In the previous section it was shown that the identification of all the parameter values of the two-compartment model requires sampling of the plasma activities at least upto 10 days after AMI and the inclusion of a very slowly eliminated enzyme in the analysis. While this first requirement presents merely an incon- 
Table 3.9 Estimation of the values of the circulatory parameters of the one-compartment model from data calculated for the two-compartment model.

\begin{tabular}{|c|c|c|c|c|c|c|c|c|}
\hline & & TER & $\mathrm{ERR}$ & rho & $\mathrm{FCR}_{1}$ & $\mathrm{rhO}_{2}$ & $\mathrm{FCR}_{2}$ & Ress \\
\hline Refe & rence values & 0.014 & 0.018 & 1.0 & 0.0150 & 0.140 & 0.20 & - \\
\hline Fit & $0 \leqq T \leq 360$ & - & - & 11.0 & 0.0096 & 0.225 & 0.075 & 7.6 \\
\hline & $0 \leq \mathbb{T} \leq 240$ & - & - & 1.0 & 0.0408 & 0.219 & 0.086 & 6.7 \\
\hline & $0 \leqq T \leqq 180$ & - & - & 1.0 & 0.0136 & 0.193 & 0.114 & 4.5 \\
\hline & $0 \leq T \leq 120$ & - & - & 1.0 & 0.0140 & 0.170 & 0.140 & 2.2 \\
\hline & $0 \leqq T \leqq 96$ & - & - & 1.0 & 0.0187 & 0.158 & 0.161 & 1.2 \\
\hline Refe & cence values & 0.014 & 0.018 & $2 \cdot 4$ & 0.100 & 0.140 & 0.200 & - \\
\hline Fit & $0 \leqq T \leq 96$ & - & - & 2.4 & 0.083 & 0.150 & 0.150 & 1.7 \\
\hline Refe & cence values & 0.014 & 0.018 & 2.4 & 0.100 & 1.00 & 0.300 & - \\
\hline Fit & $0 \leq \mathbb{T} \leqq 96$ & - & - & 2.4 & 0.088 & 1.11 & 0.228 & 1.6 \\
\hline Refe & cence values & 0.014 & 0.018 & 0.14 & 0.200 & 1.00 & 0.300 & - \\
\hline Fit & $0 \leqq T \leqq 96$ & - & - & 0.134 & 0.1167 & 1.00 & 0.236 & 0.6 \\
\hline
\end{tabular}

Error free data were calculated using the reference input function $(3.8)$ and the unit impulse function of the two-compartment model:

$$
P_{1} e^{-k_{1} t}+p_{2} e^{-k_{2} t}
$$

corresponalng to the reference values of the circulatory parameters. Indicated values of the estimated parameters were obtained by fitting the one-compartment analogon of (3.7) to these data restricted to the indicated time interval.

venilence because of the considerable effort to be spent in sample collection and emzyme determinations, the second is really restrictive because of the cardiac enzymes essentially only HBD (or $L D_{1}$ and $L D_{2}$ ) meets this requirement.

Therefore a simplification of the estimation procedure brought about by the analysis of the data using an one-compartment model could be of considerable interest if such a procedure would allow 
the identification of the values of FCR of rapidly eliminated enzymes without reference to sampled plasma activities of $A B D$ and if a shorter interval of data collection would suffice.

The validity of this approach is tested by fitting the onecompartmental model equivalent of (3.7) to error free data generated using the reference input function $f(t)$, cf. $(3.8)$ and the two-compartmental unit impulse function. From the data presented in Table 3.9 it appears that this procedure results in a bias in the estimates of the values of the circulatory parameters as well a considerable lack of $f$ it when a slowly eliminated enzyme is included. In the cases with only rapidly eliminated enzymes included in the fit (with FCR>0.10), the quality of the fit as measured by the deviation between model and data appears to be satisfactory. However, even in this case the bias in the estimated values of FCR is not negligible.

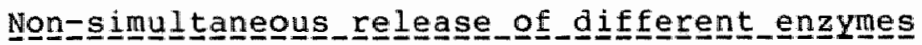

The proposed estimation procedure depends crucially on the assumption that enzyme release of the various enzymes runs strictly parallel, cf. (3.7). Although there exists much experimental evidence in support of this assumption, cf. Chapter 2.6, it would be of some consequence if the validity of this assumption could be infered independently from the simultaneously sampled data alone. Therefore we simulated data sets representing two natural departures from parallel release:

First an overall retardation (or accelleration) of the release of one of the enzymes in comparison to the release of the other enzyme(s) is simulated by replacement of the input function $f_{0}(t), c f .(3.8)$

by

$$
f_{0}(t)=c t^{2} e^{-(2 / t m) t} ; \text { with } t m=18.0
$$

$$
f_{s}(t)=C^{\prime \prime} t^{2} e^{-(2 /(t m+d t)) t} ; C^{\prime}=c(t m /(t m+d t))^{3}
$$

while the other enzymes retain the input $f_{o}(t)($, times $1 / r$ ho $j)$. Apparently the input of this selected enzyme then proceeds at a rate $t m /(t m+d t$ ) slower (if $d t>0$ ) or faster (if $d t<0$ ) than the 
Table 3.10 The effect of accelerated or retarded release of one of the enzymes on the estimated values of the circulatory parameters.

$\begin{array}{rlrl}\text { TER ERR } & \text { rhol } & \text { rhol } \\ \mathrm{FCR}_{1} & & \mathrm{rho}_{2} & \text { Res? } \\ \mathrm{FCR}_{2} & \end{array}$

\begin{tabular}{|c|c|c|c|c|c|c|c|}
\hline $\begin{array}{c}\text { Reference } \\
\text { values }\end{array}$ & 0.014 & 0.018 & 1.0 & 0.015 & 0.14 & 0.0280 & - \\
\hline$d t=-5.0$ & 0.0067 & 0.0129 & 1.0 & 0.0124 & 0.035 & 0.0239 & 1.4 \\
\hline$d t=-4.0$ & 0.0080 & 0.0140 & 1.0 & 0.0129 & 0.053 & 0.0243 & 1.0 \\
\hline $\mathrm{d} t=-3.0$ & 0.0095 & 0.0152 & 1.0 & 0.0135 & 0.072 & 0.0254 & 0.7 \\
\hline $\mathrm{at}=-2.0$ & 0.0111 & 0.0164 & 1.0 & 0.0140 & 0.092 & 0.0264 & 0.5 \\
\hline$a t=-1.0$ & 0.0130 & 0.0177 & 1.0 & 0.0147 & 0.113 & 0.0273 & 0.5 \\
\hline$a t=0.0$ & 0.0151 & 0.0190 & 1.0 & 0.0154 & 0.136 & 0.0287 & 0.6 \\
\hline$d t=1.0$ & 0.0177 & 0.0205 & 1.0 & 0.0161 & 0.160 & 0.0301 & 0.7 \\
\hline$d t=2.0$ & 0.0210 & 0.0221 & 1.0 & 0.0170 & 0.185 & 0.0315 & 0.8 \\
\hline $1 t=3.0$ & 0.0252 & 0.0240 & 1.0 & 0.0180 & 0.211 & 0.0333 & 1.0 \\
\hline$=4.0$ & 0.0308 & 0.0270 & 1.0 & 0.0194 & 0.239 & 0.0356 & .1 \\
\hline$a t=5.0$ & 0.0380 & 0.0282 & 1.0 & 0.0209 & 0.268 & 0.0383 & .2 \\
\hline
\end{tabular}

Error free data were calculated for both enzymes using the indicated reference values for the circulatory parameters and the input functions.

$$
f_{0}(t)=c t^{2} e^{-(2 / t m) t} \text {, with } t m=18 \text { and } c=0.78
$$

and

$$
f_{g}(t)=c^{\prime} t^{2} e^{-(2 /(t m+d t)) t}, c^{\prime}=c(t m /(t m+d t))^{3}
$$

for enzyme 1 and enzyme 2 respectively. Indicated data represent the parameter values estimated by fitting the two-compartment model for simultameously released enzymes, of. (3.7), to these unperturbed data. 
$\cup / 1$

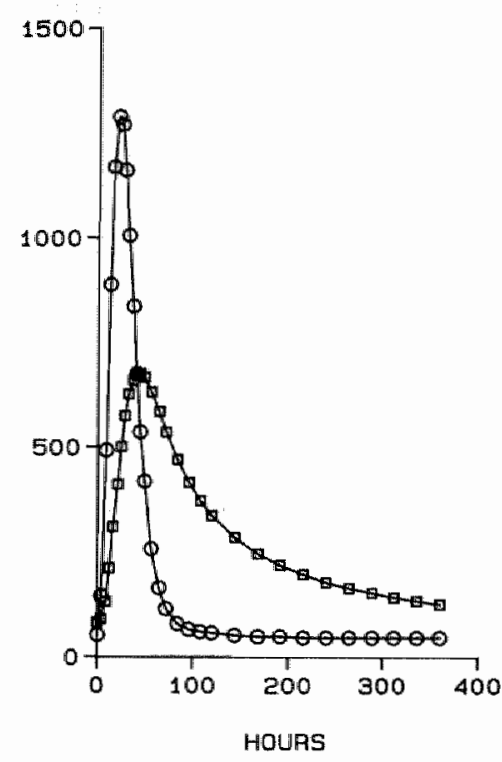

$U / 1$

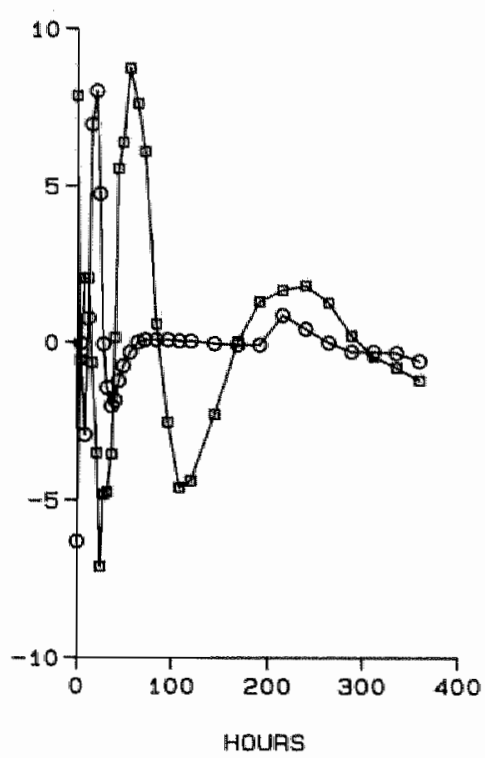

Fig. 3.8 Input $f_{2}(t) 25$ accelerated compared to $f_{1}(t)$ i.e. rho $f_{2}(t)=$ who $20 / 16 f(20 / 16 t)$.

Left panel: Fit (solid lines) and simulated data $c_{1}=a ; c_{2}=0$. Right panel: Deviation between data and fit $C_{1}(t)-M(1, p, t)=0$; $C_{2}(t)-M(2, p, t)=0$.

input as modelled by $f_{0}(t)$, while the total amount of enzyme released into the plasma is unchanged.

The data from Table 3.10 demonstrate that this kind of deviation from the model (assumptions) severely affects the validity of the estimated parameter values:

- Especially the estimated values of $\mathrm{rho}_{2}$ and also of TER and ERR exhibit a sizeable bias.

- The estimations of $\mathrm{FCR}_{1}$ and $\mathrm{rho}_{2} \cdot \mathrm{FCR}_{2}$ are affected to a much lesser extent.

- The deviation between model and data (the unexplained variation) remains small.

This relatively good quality of the fit, in presence of such model deviations is also apparent from Fig. 3.8. Although the 
residuals exhibit a systematic time courge, the size of the deviation between data and model is so small that the presence of an error in sampled data of the order of 5 will prohibit the detection of such small departure of less than 1 .

The pronounced sensitivity of the estimation procedure for such departures of the simultaneous release in combination with the unability to detect these departures from residual analysis may cast some doubts on the validity of estimated values of the circulatory parameters, in absence of an independent validation.

However in view of the data presented in Chapter 2.6 it must be expected that cytoplasmic enzymes are released in proportion to the quantities of these enzymes present in the necrotic tissue, implying equality of the ratio of cytoplasmic tissue content of the enzymes to the value of rho. A delayed (or accelerated) release then will be reflected, cf. Table 3.10 , by a deviating $v a d u e$ of tho.

In Fig. 3.9 a more physiological model for disparity of enzyme release is presented. It is assumed that with progressing necrosis enzyme is released from the cells into interstitial space at a rate of $f_{c}(t)$. The quantity $I(t)$ of enzyme present in the interstitium is assumed to be transported directly into plasma at a rate $f_{p}(t)=k_{p i} I(t)$ while part of the enzyme may also be degraded locally at a rate $k_{o i} I(t)$.

With this model the input into plasma equals

$$
E_{p}(t)=k_{p i} I(t)=k_{p i} \int_{0}^{t} e^{-\left(k_{p i}+k_{o i}\right)(t-\tau)_{E_{c}}(\tau) d \tau}
$$

In absence of detailed data on the processes involved we made the following speculative assumptions

$$
\begin{aligned}
\mathrm{f}_{\mathrm{c}}(t) & =c t \mathrm{e}^{-0.11} \cdot t \\
\mathrm{k}_{\mathrm{pi}} & =0.11 \\
\mathrm{c} & =14.1
\end{aligned}
$$

with the effect that in absence of local degradation of enzyme in the interstitium the resulting $f_{p}(t)$ equals the original reference input $(3.8)$. Otherwise $f_{p}(t)$ equals 


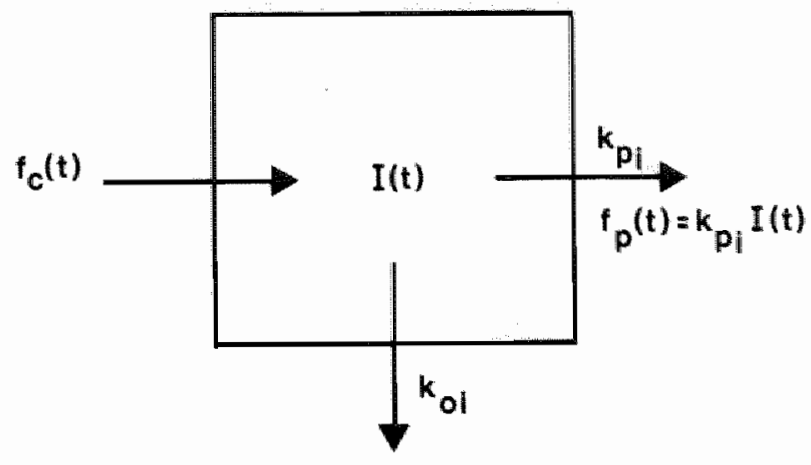

Fig. 3.9 Model for local degradation of enzyme.

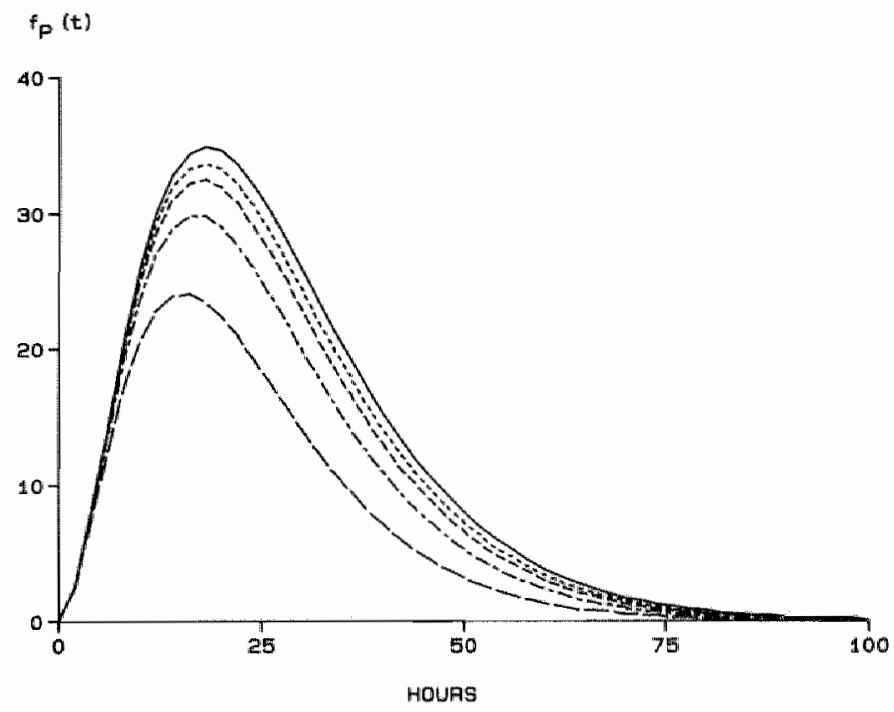

Fig. 3.10 The input function $f(t)$ as calculated for the model of tocal degradation for vamiaus values of the fraction reaching the circulation.

$F=1.0:(-) ; F=0.95:(\cdots \cdots) ; F=0.90:(-\cdots) ; \quad F=0.80:(-\cdots)$; $F=0.60:(-\longrightarrow)$. 


$$
f_{p}(t)=c t e^{-0.111 t * e^{-\left(k_{o i}+k_{p i}\right) t}}
$$

Cf. Fig. 3.10.

It should be noted that positive values of $\mathrm{k}_{\text {oi }}$, i.e. local degradation, affect the amount of enzyme reaching the circulation i.e. Only the fraction $\mathrm{F}=\mathrm{k}_{\mathrm{pi}} /\left(\mathrm{k}_{\mathrm{pi}}+\mathrm{k}_{\mathrm{oi}}\right)$ of the quantity of enzyme released by the cells reaches the circulation in this model.

Evidently different rates of local degradation, $\mathrm{k}_{\mathrm{oi}}$, for the various enzymes in consideration would even if the cellular release of these enzymes runs strictly parallel, result in a disparity of the input of these enzymes into plasma. In Table 3.11 we present the resulting estimated values of the circulatory parameters obtained by fitting on simulated data. From these data it appears that the value of $\mathrm{rho}_{2}$ is hardly affected by this kind of departure from the model, while the value of $\mathrm{FCR}_{2}$ of the rapidy eliminated enzyme is severely affected. The remaining circulatory parameters TER, ERR and even $\mathrm{FCR}_{1}$, the fractional catabolic rate of the slowly eliminated enzyme, are much less influenced. 
Table 3.11 The effect of local degradation of one of the enzyes upor the estimation of the curulatory parameters

$\begin{array}{cccccccc} & \text { TER } & \text { ERR } & \text { rho, } & \text { FCR }_{1} & \text { RHO } & \text { FCR }_{2} & \text { Res } \\ \text { Reference values } & 0.014 & 0.0180 & 1.0 & 0.0150 & 0.140 & 0.200 & -\end{array}$

Local degradation of the first enzyme

$\begin{array}{llllllll}F=1.00 & 0.015 & 0.019 & 1.0 & 0.0154 & 0.136 & 0.211 & 0.6 \\ F=0.95 & 0.016 & 0.020 & 1.0 & 0.0156 & 0.137 & 0.202 & 0.6 \\ F=0.90 & 0.017 & 0.020 & 1.0 & 0.0158 & 0.137 & 0.293 & 0.6 \\ F=0.80 & 0.018 & 0.021 & 1.0 & 0.0162 & 0.138 & 0.174 & 0.7 \\ F=0.60 & 0.021 & 0.024 & 1.0 & 0.0164 & 0.137 & 0.131 & 0.8\end{array}$

Local degradation of the second enzyme

$\begin{array}{llllllll}F=1.00 & 0.015 & 0.019 & 1.0 & 0.0154 & 0.136 & 0.211 & 0.6 \\ F=0.95 & 0.014 & 0.019 & 1.0 & 0.0151 & 0.135 & 0.220 & 0.5 \\ F=0.90 & 0.014 & 0.018 & 1.0 & 0.0149 & 0.134 & 0.231 & 0.5 \\ F=0.80 & 0.012 & 0.017 & 1.0 & 0.0145 & 0.130 & 0.260 & 0.4 \\ F=0.60 & 0.010 & 0.016 & 1.0 & 0.0139 & 0.117 & 0.372 & 0.6\end{array}$

The presented circulatory parameters are obtained by fitting the two-compartment model to error free data calculded as the convolution of the unit impulse response corresponding to the indicated reference values of the circulatory parameters and the Input function. The input function of one of the enzymes is the reference input $f_{0}(t)$ while for the other enzyme the expression for $f_{p}(t)$ (see text) is used as input function with as rate of local degradation $k_{\text {oi }}$ such that the fraction of enzyme $F$ reaching the circulation

$$
E=\left(0.11 /\left(0.11+k_{o i}\right)\right)
$$

equals the indicated value, i.e. $k_{o i}=0.11(1-F) / F$. 


\subsection{Simplified estimation procedures}

In the previous sections of this chapter it was shown that for the identification of the full set of two-compartment circulatory model parameters it is necessary to analyse plasma activity curves of two simultaneously released enzymes sampled for at. least 10 days after AMI. A further essential requirement turned out to be the inclusion of a very slowly eliminated enzyme (FCR $0.05 \mathrm{~h}^{-1}$ ) in the set op plasma activity curves considered for the estimation. This presents a severe restriction: Essentially only sets of enzymes including HBD can be analysed, because no other cardiac enzyme with sufficient slow elimination rate is known. It would be of some interest if one could determine routinely the FCR's of a rapidly eliminated enzymes by a less exigent procedure and more fundamentally if it would be possible to check the FCR's of rapidly eliminated enzymes obtained by estimation in combination with HBD by an independent method.

In this section two procedures are proposed to meet these requirements. The first of these procedures extends our abservations in section 5 of this chapter on the employment of the one-compartmental model for the analysis of data of enzymes with a catabolic rate much larger than the extravasation rate. The proposed method consists in assuming fixed values for $T E R$ and ERR (these parameters cannot be estimated when considering only enzymes with FCR TER, cF. Section 3.4) and reduction of the data used for the analysis to 4 days after onset of AMI.

obviously the results of such a procedure depend on the supplied values of TER and ERR and the quality of data.

Therefore this procedure is evaluated by using simulated data, constructed as described in section 4 of this chapter and contaminated with 5 error. A further data set was constructed by this procedure with random values for TER and ERR, Gaussian distributed with mean equal to the indicated reference values in Table 3.12 and with a variance corresponding to a coefficient of variation of $20 \%$, the anticipated biological variation in TER and ERR.

In Table 3.12 the results of estimation with TER and ERR kept fixed at various values are presented. From these data it appears 
Table 3.12 Estimation of the FCR"s of rapialy eliminated enzyes by simultaneous analysis of two simulated plasma activty curves contaminated with 5 error with the two-compartmental model supplied with fixed values of TER and ERR.

\begin{tabular}{|c|c|c|c|c|c|c|}
\hline & TER & $\mathrm{ERR}$ & rho, & $\begin{array}{c}\text { rho, } \\
\text { FCR }\end{array}$ & $\mathrm{rho}_{2}$ & $\begin{array}{l}\mathrm{Tho}_{2} \\
\mathrm{FCR}_{2}\end{array}$ \\
\hline eference values & 0.0140 & 0.0180 & 2.4 & 0.240 & 0.140 & 0.0280 \\
\hline
\end{tabular}

\begin{tabular}{|c|c|c|c|c|c|c|}
\hline $\begin{array}{l}\text { Fixe } \\
\text { for }\end{array}$ & $\begin{array}{l}\text { d values } \\
\text { TER and ERR }\end{array}$ & Res? & $\mathrm{rho}_{1}$ & rho ${ }_{1}$ & $\begin{array}{l}\text { Who } \\
\text { FCR, }\end{array}$ & $\begin{array}{l}\text { rho }_{2} \\
\mathrm{ECR}_{2}\end{array}$ \\
\hline TER & $=0.0093$ & 4.9 & 2.4 & 0.209 & 0.143 & 0.0244 \\
\hline ERR & $=0.0080$ & 0.9 & - & 0.048 & 0.020 & 0.0054 \\
\hline TER & $=0.0093$ & 4.9 & 2.4 & 0.219 & 0.142 & 0.0251 \\
\hline ERR & $=0.0120$ & 0.9 & - & 0.049 & 0.020 & 0.0055 \\
\hline TER & $=0.0093$ & 4.9 & 2.4 & 0.229 & 0.140 & 0.0264 \\
\hline ERR & $=0.0180$ & 0.9 & - & 0.051 & 0.020 & 0.0057 \\
\hline TER & $=0.0140$ & 4.9 & 2.4 & 0.231 & 0.140 & 0.0271 \\
\hline ERR & $=0.0120$ & 0.9 & - & 0.053 & 0.020 & 0.0053 \\
\hline TER & $=0.0140$ & 4.9 & 2.4 & 0.246 & 0.136 & 0.0286 \\
\hline ERR & $=0.0480$ & 0.9 & - & 0.055 & 0.021 & 0.0062 \\
\hline TER & $=0.0140$ & 4.9 & 2.4 & 0.257 & 0.135 & 0.0295 \\
\hline ERR & $=0.0270$ & 0.9 & - & 0.057 & 0.021 & 0.0063 \\
\hline TER & $=0.0210$ & 4.9 & 2.4 & 0.273 & 0.131 & 0.0320 \\
\hline ERR & $=0.0180$ & 0.9 & - & 0.063 & 0.022 & 0.0071 \\
\hline TER & $=0.0210$ & 4.9 & 2.4 & 0.290 & 0.130 & 0.0335 \\
\hline ERR & $=0.0180$ & 0.9 & - & 0.065 & 0.022 & 0.0072 \\
\hline TER & $=0.0210$ & 4.9 & 2.4 & 0.292 & $0.13 \|$ & 0.0334 \\
\hline ERR & $=0.0410$ & 0.9 & - & 0.064 & 0.022 & 0.0071 \\
\hline
\end{tabular}

Results obtained with simulated data constructed with random values for TER and ERR, see text:
$\operatorname{TER}=0.014 \pm 0.0028$
4.9
$2.4 \quad 0.251$
0.136
0.0290
$\mathrm{ERR}=0.018 \pm 0.0036$
0.9
$-0.077 \quad 0.021$
0.0086

Indicated figures are the mean \pm SD of 100 fits on independently aimulated data sets. Data up to 96 hours were used for the estimation. 
that the bias introduced by using incorrect values of TER and ERR in this estimation procedure remains rather restricted: a 50 : deviation in TER and ERR causes only a bias of maximally 8 in the estimation of tho and only 20 in the estimation of the rho. FCR's. Comparison with the results in the last entry of this table presenting the results of estimation with the correct mean values of TER and ERR on data simulating the 20 interindividual variation in TER and ERR values shows that such a variation of TER and ERR hardly contributes to the variation in the estimates.

The second method is based on the distinguised position of HBD, with $\mathrm{FCR}=0.015, \mathrm{TER}=0.014, \mathrm{ERR}=0.018$, anong the cardiac enzymes because of its extremely slow rate of elimination. For such an enzyme the plasma activity reflects during the initial phase of enzyme relase into the plasma with some precision the integrated input into plasma. In order to calculate the total cumulative input into plasma, cf. Table A.2 only small corrections accounting for the elimination and extravasation must be made. Hence it seems appropriate to use the plasma activities of HBD with fixed mean values of the circulatory parameters of HBD to estimate the input function $f(t)$. The remaining parameters, the rho and FCR for each rapidly eliminated enzyme must then be estimated by a simultaneous analysis of the plasma activity curve of this enzyme and of HBD.

Practically this procedure is implemented by fitting the model function with fixed mean walues of the circulatory parameters of HBD to the initial stage (say up to 36 or 48 hours) of the enzyme in study. Thus this method is only applicable after the determination of the circulatory parameters of the reference enzyme.

Obviously ith this procedure one introduces beyond the sampling error also an error in the estimation due to the neglection of the biological variation between individuals in the circulatory parameters of the reference enzyme. The impact of this neglection of the interindividual variation of these parameters by using fixed mean values instead of the individual parameter values is anticipated to be small as the contribution to the calculated cumulative release of terms containing these parameters remains small up to 48 hours. 
In order to evaluate this procedure we simulated plasma activity curves of the reference enzyme using random values from a Gaussian distribution with mean value equal to the indicated reference value and a standard deviation equal to the anticipated biological variation in the values of the circulatory parameters, 20\%, cf. Table 2.4 for FCR, TER and ERR and Table 4.2 for CS:

$$
\begin{array}{ll}
\mathrm{CS}(\mathrm{HBD}): \text { mean }=80 ; & ; \mathrm{SD}=16 \\
\mathrm{FCR}(\mathrm{HBD}) & : \text { mean }=0.015 ; \mathrm{SD}=0.0030 \\
\mathrm{TER} & : \text { mean }=0.014 ; \mathrm{SD}=0.0028 \\
\mathrm{ERR} & : \text { mean }=0.018 ; \mathrm{SD}=0.0036
\end{array}
$$

Beyond this the procedure to construct data for simulations as described in Section 4 of this chapter was followed. Sampling error of 58 was simulated by adding a random number to the data.

In Table 3.13 the results of estimation of rho and FCR of rapidly eliminated enzymes by this procedure applied to such simulated data are presented. From these data it appears that plasma activity curves sampled up to 36 hours suffice to obtain rho and FCR with a satisfactory precision although the variance in the estimation can be reduced by using data up to 48 hours. 
Table 3.13 Estmation of the rex and tho of a rapidly eliminated enzwe with HED as a reference emzyme Results are obtained by fitting data for different time interwals and for various reference values of FCR using 100 simulated data sets, see text.

Res tho rho.FCR

Reference values

$$
\begin{aligned}
& 24 \text { in mean } \\
& \text { S.D. }
\end{aligned}
$$

$36 \mathrm{~h}$

$$
\text { mean }
$$

S. D.

$48 \mathrm{~h}$

$$
\text { mean }
$$

S.D.

Reference vallues

$24 \mathrm{~h}$ mean
S.D.

$36 \mathrm{~h}$

$$
\text { mean }
$$

S.D.

$48 h$

mean

S.D.

Reference values

$24 \mathrm{~h}$ mean
$5 . D^{*}$

$36 \mathrm{~h}$

$$
\text { mean }
$$

S.D.

$48 \mathrm{~h}$ mean

$48 \mathrm{~h}$

$$
\begin{aligned}
& \text { mean } \\
& \text { S.D. }
\end{aligned}
$$

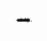

4.7

$2 \cdot 2$

4. 8

1.5

5.0

1.1

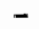

4.7

2.2

4. 6

1.7

5. 0

1.3

$-$

5.0

2.0

4.9

1.5

5. 0

1. 5

5.0

1.2

cf. Mable 3.12
1.07
0.23

1.07

0. 31

0.295

0.024

$$
2.4 \quad 0.24
$$

$\begin{array}{ll}2.51 & 0.224 \\ 0.45 & 0.054\end{array}$

2.48

0.230

0.032

$\begin{array}{ll}2.43 & 0.235 \\ 0.24 & 0.019\end{array}$

0.114

0.0280

$\begin{array}{ll}0.150 & 0.0263 \\ 0.032 & 0.0038\end{array}$

$0.147 \quad 0.0269$

0.027

0.0025

0.143

0.0269

0.020

0.0018

1.00

0.300

1. 16

0.284

0.034

1. 11

0.291

0.31

0.024

0.295

0.019 


\section{APPENDIX B}

B. 1. Identifiability_of_the_unit__impulse_response_from_simultane ous Iy_sampled_plasma_activity_curves

The multiple system response upon an unknown input $f(t)$ is given by

$$
c_{j}(t)=\left(1 / r h o_{j}\right) P_{b j}(t) * f(t), j=1, \ldots, m
$$

with $P_{b j}(t)$ the unit impulse response of enzyme $j$. Laplace transformation of this formula results in:

$$
\overline{\mathrm{C}}_{j}(s)=\left(1 / \mathrm{rho} o_{j}\right) \overline{\mathrm{P}}_{\mathrm{bj}}(\mathrm{s}) \overline{\mathrm{f}}(\mathrm{s}) ; j=1, \ldots, \mathrm{m}
$$

In order to avoid the trivial undeterminacy brought about by the freedom to scale $f(t)$, we assume from now on that rho $1=1$. The quatients

$$
\overline{\mathrm{r}}_{j \ell}(\mathrm{s})=\overline{\mathrm{C}}_{j}(\mathrm{~s}) / \overline{\mathrm{C}}_{\ell}(\mathrm{s})=\left(\overline{\mathrm{P}}_{\mathrm{bj}}(\mathrm{s}) / \mathrm{rho} \mathrm{o}_{j}\right) /\left(\overline{\mathrm{P}}_{\mathrm{b} \ell}(\mathrm{s}) / \mathrm{rho} \mathrm{o}_{\ell}\right), \begin{array}{r}
j=1, \ldots, \mathrm{m} \\
1=1, \ldots, \mathrm{m}
\end{array}
$$

are independent of the unknown $f(t)$ and contain essentially all the information on $\mathrm{P}_{\mathrm{bj}}$ obtainable from the sampled plasma curves.

The one-compartment model

In this case the mono-exponential unit impullse response is given by

$$
P_{b j}(t)=e^{-k_{1 j} t},\left(k_{1 j}=F C R_{j}\right)
$$

and thus

$$
\left.\bar{r}_{j l}(s)=\left(1 / r h o_{j}\right) \frac{s+F R_{1}}{s+\operatorname{FCR}_{j}}=\left(1 / r h o_{j}\right)\left(1+\frac{\mathrm{FCR}_{1}-\mathrm{FCR}_{j}}{s+\mathrm{FCR}_{j}}\right)\right)
$$

clearly both the ratio rhoj and the FCR"s of both enzymes are defined by the $\vec{r}_{j l}(s)$. 
The_two=compartment_model

The bi-exponential unit impulse response $P_{b j}(t)$ is given by (3.3) and the Laplace transformation of $P_{b j}(t)$ equals, cf. (A.8):

$$
P_{b j}(s)=\left(s+\mathbb{E} R R_{j}\right) /\left(s+k_{1 j}\right)\left(s+k_{2 j}\right)
$$

(with the substitution $\operatorname{ERR}_{j}=k_{e}, k_{1 j}=k_{1}$ and $k_{2 j}=k_{2}$ in $(A .8)$ ). One finds

$$
\bar{r}_{j 1}(s)=1 / \text { cho } o_{j} \frac{\left(s+E_{j}\right)\left(s+k_{11}\right)\left(s+k_{21}\right)}{\left(s+E_{j}\right)\left(s+k_{1 j}\right)\left(s+k_{2 j}\right)}
$$

Although this expression enables one to identify the sets

$$
\begin{aligned}
& A=\left\{a_{1}, a_{2}, a_{3}\right\}=\left\{E_{j}, k_{11}, k_{21}\right\} \\
& B=\left\{b_{1}, b_{2}, b_{3}\right\}=\left\{E_{1}, k_{1 j}, k_{2 j}\right\}
\end{aligned}
$$

there remains however an ambiguity in the assignment of the correspondence of one of the $a_{j}$ to the walue of $E R R_{j}$.

In the case that only two responses are analysed simultaneously this unidentifiability is unresolvable and even the requirement that the circulatory parameters (cf. A.14)

and

$$
\mathrm{FCR}_{j}=\mathrm{k}_{1 j} \mathrm{k}_{2 j} / \mathrm{ERR}
$$

$$
\operatorname{TER}_{j}=-\left(k_{1 j}-E R R_{j}\right)\left(k_{2 j}-E R R_{j}\right) / E R R_{j}
$$

must be positive (physical realisability) cannot resolve this unidentifiability as is demonstrated by the following example: given the values $a_{1}<b_{1}<a_{2}<b_{2}<a_{3}<b_{3}$ both choices

and

$$
\text { (1) } \begin{aligned}
k_{11}=a_{1}, k_{21}=a_{3}, E R R_{1}=b_{2} \\
k_{1 j}=b_{1}, k_{2 j}=b_{3}, E R R_{j}=a_{2}
\end{aligned}
$$

(2) $k_{11}=a_{1}, k_{21}=a_{2}, E_{1} R_{1}=b_{1}$

$$
k_{1 j}=b_{2}, k_{2 j}=b_{3}, E R R_{j}=a_{3}
$$


are admissible with respect to physical realisability "Certainly, this ambiguity might in principle be resolved if more than two enzymes are analysed simultaneously:

Both ratios

$$
\bar{r}_{j \perp}(s)=\bar{c}_{j}(s) / \bar{c}_{1}(s)=\frac{1}{r o_{j}} \frac{\left(s+E R R_{j}\right)\left(s+k_{11}\right)\left(s+k_{21}\right)}{\left(s+E R R_{1}\right)\left(s+k_{1 j}\right)\left(s+k_{2 j}\right)}
$$

and

$$
\bar{r}_{j \ell}(s)=\bar{c}_{j}(s) / \bar{c}_{\ell}(s)=\frac{r o_{\ell}}{r o_{j}} \frac{\left(s+E_{j}\right)\left(s+k_{1 \ell}\right)\left(s+k_{2 \ell}\right)}{\left(s+E_{\ell}\right)\left(s+k_{1 j}\right)\left(s+k_{2 j}\right)}
$$

share the (only) common factor (s+ERR, in the numerator and hence the $a_{j}$ representing the value of $E R R_{j}$ may be identified. This procedure is however not practical because its sensitivity to perturbations caused by sampling errors.

A significant simplification is attained if it is assumed that for the enzymes considered the values of TER and ERR are identical. Then

$$
\bar{r}_{j I}(s)=1 / \text { rho }_{j} \frac{\left(s+k_{11}\right)\left(s+k_{21}\right)}{\left(s+k_{1 j}\right)\left(s+k_{2 j}\right)}
$$

which can be rewritten as, (cf. A.14)

$$
\bar{r}_{j I}(s)=1 / \mathrm{rho}_{j}\left(1+\left(\mathrm{FCR}_{1}-\mathrm{FCR}_{j}\right)\left(\frac{\mathrm{P}_{1 j}}{s+\mathrm{k}_{1 j}}+\frac{\mathrm{P}_{2 j}}{s+\mathrm{k}_{2 j}}\right)\right)
$$

From this expression (B.3b) the circulatory parameters are determined for $\mathrm{ECR}_{1}$ unequal to $\mathrm{FCR}_{j}$ :

$\mathrm{P}_{1 j}, \mathrm{P}_{2 j}, \mathrm{k}_{1 j}$ and $\mathrm{k}_{2 j}$ Eully characterize the circulatory model of enzyme $j$, cf. Formula $(A .14)$, and thus $\mathrm{FCR}_{j}$, TER and ERR, while the ratio cho $j$ and the difference $F C R_{1}-F^{-} R_{j}$ also are determined from (B.3b).

The three-compartment model

In an analogeous way as with the general two-compartment model it can be demonstrated that the general three-compartment model leads to unidentifiability unless the circulatory models for the various enzymes are restricted. One such an restriction is again presented by the assumption that the kinetics of the plasma 
levels can be described by Mathews mamilary model with exchange rates between the plasma and the extravascular pools identical for all enzymes involved, which hence differ only with respect to the elimination rate $\mathrm{FCR}_{j}$. Then

$$
\bar{r}_{j 1}(s)=\bar{c}_{j}(s) / \bar{c}_{1}(s)=1 / \text { rho } \frac{\left(s+k_{11}\right)\left(s+k_{21}\right)\left(s+k_{31}\right)}{\left(s+k_{1 j}\right)\left(s+k_{2 j}\right)\left(s+k_{3 j}\right)}
$$

which may be rewritten as

$$
\bar{r}_{j 1}(s)=1 / \operatorname{cho}_{j}\left(1+\left(\mathrm{FCR}_{1}-\mathrm{FCR}_{j}\right)\left(\frac{\mathrm{P}_{1 j}}{s+k_{1 j}}+\frac{\mathrm{P}_{2 j}}{s+\mathrm{k}_{2 j}}+\frac{\mathrm{P}_{3 j}}{s+\mathrm{k}_{3 j}}\right)\right)
$$

which clearly allows the identification of the circulatory parameters of enzyme $j$ together with $\mathrm{rho}_{j}$ and $\mathrm{FCR}_{1}-\mathrm{FCR}_{j}$.

B. 2. Numerical_implementation_of_the_estimation_procedure._Calcu=

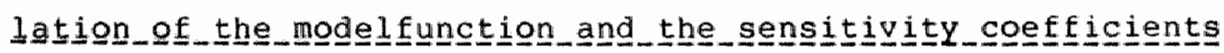

The modelfunction $M(j, p, t)$, defined in Formula $(3.7)$, equals

$$
M(j, p, t)=C S_{j}+1 / r h_{j} \sum_{i=1}^{N_{S}} F_{i}\left(P_{1 j} h_{i}\left(k_{1 j}, t\right)+P_{2 j} h_{i}\left(k_{2 j}, t\right)\right)
$$

with

$$
\begin{aligned}
& h_{i}(k, t)=\int_{0}^{t} s_{i}(\tau) e^{-k(t-\tau)} d \tau \\
& k_{1 j}, k_{2 j}=\frac{1}{2}\left(\mathrm{FCR}_{j}+\mathrm{TER}+\mathrm{ERR} \pm \sqrt{\left(\mathrm{FCR}_{\mathrm{j}}+\mathrm{TER}+\mathrm{ERR}\right)^{2}-4 \mathrm{FCR}_{\mathrm{j}} \mathrm{ERR}},\right. \\
& P_{1 j}=\left(k_{1 j}-E R R\right) /\left(k_{1 j}-k_{2 j}\right) ; P_{2 j}=\left(k_{2 j}-E R R\right) /\left(k_{2 j}-k_{1 j}\right) \\
& s_{i}(t)=\sum_{\ell=-1}^{1} w_{i \ell}\left(t-t s_{i+\ell}\right) ; w_{i \ell}=\prod_{m=-1}^{1}\left(t s_{i+m}-t s_{i+\ell}\right)^{-1} \\
& m \neq l
\end{aligned}
$$

Hence one may express $M$ in terms of the function $\mathrm{Ct}_{+}$defined by

$$
\begin{array}{rlrl}
c t+\left(t-t s_{i}, k\right) & =\int_{0}^{t}\left(\tau-t s_{i}\right)+e^{-k(t-\tau)} d \tau \\
& =\int_{0}^{t} \tau \quad e^{-k\left(t-t s_{i}-\tau\right) d \tau ; t-t s_{i} \geqq 0} \\
& =0 & ; t-t s_{i}<0
\end{array}
$$


Thus the expression

$$
\begin{aligned}
& M(j, p, t) \\
& =C s_{j}+1 / \operatorname{rho}_{j} \sum_{i=1}^{N_{s}} \sum_{i=1}^{2} \sum_{\ell=-1}^{1} F_{i} \mathbb{P}_{m j} w_{i l} c t+\left(t-t s_{i}+k_{m j}\right)
\end{aligned}
$$

is obtained for the modelfunction.

The evaluation of the function $c t,(t, k)$ requires some care. By partilal integration one finds:

$$
\begin{aligned}
c t_{f}(t, k) & =\int_{0}^{t} \tau e^{-k(t-\pi)} d \tau \\
& =\left(1 / k^{2}\right)\left(k t-1+e^{-k t}\right)
\end{aligned}
$$

But this last expression is inaccurate for $|k t| \ll 1$. In that case the series expansion

$$
\begin{aligned}
c t+(t, k) & =e^{-k t} \int_{0}^{t} \tau e^{k \tau} d \tau=e^{-k t} \int_{0}^{t} \sum_{n=0}^{\infty} \tau\left(\frac{k \tau}{n !}\right)^{n} d \tau \\
& =e^{-k t} \sum_{n=0}^{\infty} \frac{k^{n} t^{n+2}}{n !(n+2)}
\end{aligned}
$$

is more suitable; with 13 terms and $|k t|<0.5$ the resulting relative error is less than $10^{-15}$.

The calculation of the sensitivity coefficients, i.e. the derivatives of the modelfunction with respect to the (model) parameters is relatively straightforward by using formula (B.5). The function $M$ is linear in the parameters $C_{j}, E_{i}, i=1, \ldots, N_{s}$

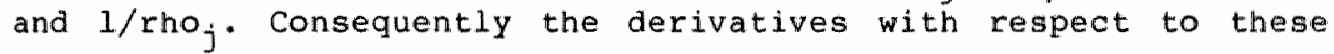
paraneters are easily calculated:

$$
\begin{aligned}
& \frac{\partial}{\partial C s_{i}} M(j, p, t)=1 \\
& \frac{\partial}{\partial F i} M(j, p, t)=1 / r h o_{j} \sum_{m=1}^{2} \sum_{\ell=-1}^{1} P_{m j} h_{1 \ell} c t_{+}\left(t-t s_{j+\ell}, k_{m j}\right) \\
& \frac{\partial}{\partial r h o_{j}} M(j, p, t)=-1 / r h o_{j}\left(M(j, p, t)-C s_{j}\right)
\end{aligned}
$$

The derivatives with respect to the parameters $x=E C R_{j}$ " "TER or 
ERF are equal to

$$
\begin{aligned}
\frac{\partial}{\partial x} M(j, p, t)= & 1 / \operatorname{cho}_{j} \sum_{i=1}^{N_{s}} \sum_{i=1}^{2} \sum_{\ell=-1}^{1} F_{i} W_{i \ell}\left(\frac{\partial}{\partial x} P_{m j}\right) c t_{+}\left(t-t s_{i+\ell}, k_{m j}\right) \\
& \left.+\left(P_{m j}\left(\frac{\partial}{\partial x} k_{m j}\right)\right) \frac{\partial}{\partial k_{m j}} c t\left(t-t s_{i+\ell}, k_{m j}\right)\right\} \quad(B .7 b)
\end{aligned}
$$

with the decivatives $\left(\frac{\partial}{\partial x} P_{m j}\right)$ and $\left(\mathbb{P}_{m j} \frac{\partial}{\partial x} k_{m j}\right)$ given in Table B.1.

Table B.1 The derivatives of the coefficients of the bi-exponential impulse response with respect to the circulatory

\begin{tabular}{|c|c|c|c|c|}
\hline$\frac{\partial}{\partial x}$ & $\frac{\partial}{\partial x} P_{1}$ & $\frac{\partial}{\partial x} P_{2}$ & $P_{1} \frac{\partial}{\partial x} k_{1}$ & $P_{2} \frac{\partial}{d x} k_{2}$ \\
\hline$\frac{\partial}{\partial \mathrm{FCR}}$ & $-\frac{2 P_{1} P_{2}}{\left(k_{2}-k_{1}\right)}$ & $-\frac{2 P_{1} P_{2}}{\left(k_{1}-k_{2}\right)}$ & $\mathbb{P}_{1}{ }^{2}$ & $\mathrm{P}_{2}{ }^{2}$ \\
\hline$\frac{\partial}{\partial \mathrm{TER}}$ & $\frac{P_{2} k_{1}-P_{1} k_{2}}{\left(k_{2}-k_{1}\right)^{2}}$ & $\frac{\mathbb{P}_{1} k_{2}-P_{2} k_{1}}{\left(k_{2}-k_{1}\right)^{2}}$ & $-\frac{P_{1} k_{1}}{k_{2}-k_{1}}$ & $-\frac{\mathrm{P}_{2} \mathrm{k}_{2}}{\mathrm{k}_{1}-\mathrm{k}_{2}}$ \\
\hline$\frac{\partial}{\partial E R R}$ & $\frac{\operatorname{TER}\left(\mathrm{k}_{1}+\mathrm{k}_{2}\right)}{\left(\mathrm{k}_{2}-\mathrm{k}_{1}\right)^{3}}$ & $\frac{\operatorname{TER}\left(k_{1}+k_{2}\right)}{\left(k_{1}-k_{2}\right)^{3}}$ & $\frac{T E R k_{1}}{\left(k_{2}-k_{1}\right)^{2}}$ & $\frac{\mathrm{TER} \mathrm{k}_{2}}{\left(\mathrm{k}_{1}-\mathrm{k}_{2}\right)^{2}}$ \\
\hline
\end{tabular}
model parameters.

The coefficients of the bi-exponential unit impulse response

$$
P_{b}(t)=P_{1} e^{-k_{1} t}+P_{2} e^{-k_{2} t}
$$

of the simplified two-compartment model are differentiated with respect to FCR, TER and ERR by using the relations

$$
\begin{aligned}
& \mathrm{P}_{1}+\mathrm{P}_{2}=1 ; \mathrm{P}_{1} \mathrm{k}_{2}+\mathrm{P}_{2} \mathrm{k}_{1}=\mathrm{ERR} \\
& \mathrm{k}_{1}+\mathrm{k}_{2}=\mathrm{FCR}+\mathrm{TER}+\mathrm{ERR} ; \mathrm{k}_{1} \mathrm{k}_{2}=\mathrm{FCR} \mathrm{ERR}
\end{aligned}
$$


The function $\frac{\partial}{\partial \mathrm{k}} c t_{+}(t, k)$ is calculated by direct differentiation of $(B .6 a)$ :

$$
\begin{aligned}
\frac{\partial}{\partial k} c t_{+}(t, k) & =-\left(2 / k^{3}\right)\left(k t-1+e^{-k t}\right)+\left(1 / k^{2}\right)\left(t-t e^{-k t}\right) \\
& =-(t+2 / k) c t_{+}(t, k)+t^{2} / k
\end{aligned}
$$

Again this formula is inaccurate for $|\mathrm{kt}| \ll 1$ and for such small values of $|\mathrm{kt}|$ better accuracy is attained by differentiation of the series expansion (B.6b):

$\frac{\partial}{\partial k} \quad c t+(t, k)=-e^{-k t} \sum_{n=0}^{\infty} \frac{k^{n} t^{n+3}}{n !(n+2)(n+3)}$

The minimization_of the sum of squared_residuals

A modified Gauss-Newton method is used in order to find a parameter set $\mathrm{p}_{e}$ which minimizes the sum of squared residuals (cf. 3.8$)$ :

$$
\operatorname{SSR}(p)=\sum_{i=1}^{N m}\left(r_{i}(p)\right)^{2}
$$

with the residuals renumbered with a single index

$$
r_{i j} \rightarrow r_{i+(j-1) N}
$$

With the notation

$\mathrm{p}$ - the column vector of the parameters $\mathrm{P}_{1}, \ldots, \mathrm{P}_{\mathrm{N}_{\mathrm{p}}}$

$r$ - the column vector of the residuals

$J$ - the Jacobian matrix of $r$, i.e.

$J_{i j}=\frac{\partial}{\partial P_{j}} r_{i}(p) ; i=1, \ldots, N m ; j=1, \ldots, N_{p}$

$g$ - the gradient vector of $\operatorname{SSR}(p), i . e$.

$g_{j}=2 \underset{i=1}{\sum_{i}^{N m}} r_{i}(p) \frac{\partial}{\partial P_{j}} r_{i}(p) ; j=1, \ldots, N_{p}$

$\mathrm{H}$ - the Hessian of $\operatorname{SSR}(\mathrm{p})$, i.e. 


$$
\begin{array}{r}
H_{j k}=2 \sum_{i=1}^{N N_{i}} r_{i}(p) \frac{\partial^{2}}{\partial p_{j} \partial p_{k}} r_{i}(p)+\frac{\partial}{\partial p_{j}} r_{i}(p) \frac{\partial}{\partial p_{k}} r_{i}(p) \\
j=1, \ldots, N_{p}, k=1, \ldots, N_{p}
\end{array}
$$

the Gauss-Newton method consists in the interative construction of a sequence of approximations $\mathbb{p}^{(k)}$ of the minimum satisfying

$$
2 J^{+} J\left(p^{(k)}-p^{(k-1)}\right)=-g\left(p^{(k-1)}\right)
$$

This is a simplification of Newton's iterative procedure for seeking a zero of the gradient $g(p)$, where the Hessian $B$ is replaced by the approximation $2 \mathrm{~J}^{+} \mathrm{J}$ neglecting the second order derivatives.

In the implementation used in this thesis the direction, $x^{(k)}$ $=p^{(k)}-p^{(k-1)}$, as defined by $(B .9)$ is retained but the stepsize is adjusted, i.e.

$$
p^{(k)}=p^{(k-1)}+\alpha^{(k)} x^{(k)}
$$

so that the sequence $\operatorname{SSR}\left(\mathrm{p}^{(\mathrm{k})}\right)$ is monotonically decreasing. Hereto the stepsize is determined by a safequarded quadratic interpolation according to a strategy originally proposed by Box and Kamenescu, cf. \Beck, 1977》.

In the actual. implementation the search direction $x^{(k)}$ is determined by solving the equivalent set of overdetermined linear equations

$$
J\left(p^{(k-1)} x^{(k)}=-r\left(p^{(k-1)}\right)\right.
$$

with help of the subroutine F04AMF from the Fortran library of [NAGLIB]. The interation is stopped and the $k$-th iterate $p^{(k)}$ is accepted as a close approximation of the minimizing $p_{e}$ if:

$$
\left\{\sum_{i=1}^{N_{p}}\left(\frac{\alpha^{(k)} x_{i}^{(k)}}{\mathbb{P}_{i}^{(k)}}\right)^{2}\right\}^{\frac{1}{2}}<10^{-4}
$$


i.e. If the relative changes in all components of the parameter vector are smaller than $10^{-4}$.

B.3 Stat isticical analys is

The estimation problem posed in chapter 3.2 concerns the estimation of the parameter vector

$$
\begin{aligned}
\mathrm{p}= & \left(\mathrm{p}_{1}, \ldots, \mathrm{p}_{\mathrm{Np}}\right)=\left(\mathrm{CS}_{1}, \ldots, \mathrm{CS}_{\mathrm{m}}, \mathrm{rho}_{2}, \ldots, \mathrm{rho}_{\mathrm{m}}, \mathrm{FCR}_{1},\right. \\
& \left.\ldots, \mathrm{FCR}_{\mathrm{m}}, \mathrm{TER}, \mathrm{ERR}, \mathrm{F}_{1}, \ldots, \mathrm{F}_{\mathrm{Ns}}\right)
\end{aligned}
$$

from comparison of the measured data, contaminated with (sampling) error $E_{i j}$, to the modelfunction (3.7) i.e.

$$
\begin{aligned}
C d_{i j}=M(j, p, t i)+\varepsilon_{i j} ; i & =1, \ldots, N \\
j & =1, \ldots, m
\end{aligned}
$$

Maximum 1ikelihood_estimator

In order to obtain an identifiable problem one must a priori specify assumptions concerning the errors $\varepsilon_{i j}$; attributing most of the sampling error to the (spectrophotometric) assay of the enzyme activity (that includes dilution of the plasma samples for high activities) errors are assumed to be independent normal variates

with

$$
\varepsilon_{i j} \sim N\left(0, \sigma^{2} a_{i j}^{2}\right)
$$

$$
\begin{aligned}
\sigma_{i j} & =c_{i j} \text { for } c_{i j} \geq 50 \mathrm{U} / \mathrm{L} \\
& =50 \text { for } c_{i j} \leqq 50 \mathrm{U} / \mathrm{L}
\end{aligned}
$$

i.e. errors are percentual except for low activites. The Log Likelihood function, cf. [Kendal (Ch. 18), 1973] of (B.10) is given by

$$
\log L=-\frac{1}{2} n \log (2 \pi)-\frac{1}{2} n \log \left(\sigma^{2}\right)-\frac{1}{2 \sigma^{2}} \sum_{\ell=1}^{n} r_{\ell}^{2}(p)
$$

with $\mathrm{n}$ - the total number of observations $\mathrm{n}=\mathrm{N} \cdot \mathrm{m}$ 


$$
\begin{aligned}
& r_{\ell}-\text { the residuals, } c f_{*} \text { Ch } 3.3, \\
& \qquad r_{i+(j-1) N}(p)=\left(1 / o_{i j}\right)\left(C d_{i j}-M\left(j, p, t_{1}\right)\right) .
\end{aligned}
$$

It follows that the least squares estimator, proposed in $\mathrm{Ch} .3 .3$, is a Maximum Likelihood Estimator under the above mentioned assumptions on the errors if the weights are choosen equal to

$$
w_{i j}=1 / a_{i j}
$$

Approximate $\underline{\text { linearized_analysis }}$

As this multiparameter non-linear estimation problem resists the analysis of the small sample properties of the least squares estimator, the corresponding linearized least squares estimation problem is used to obtain approximate results. If $\mathrm{p}^{\star}$ is the true value of the parameter vector and $p^{e}$ the least squares estimate we consider

$$
C d_{i j}=M\left(j, p^{e}, t_{i}\right)+\frac{\partial}{\partial p} M\left(j, p^{e}, t_{i}\right)\left(p-p^{*}\right)+\varepsilon_{i j}
$$

which is equivalent to

$$
y=\operatorname{Adp}+\varepsilon
$$

with $\mathrm{y}_{\mathrm{i}}=\mathrm{r}_{\mathrm{i}} ; \mathrm{dp}=\left(\mathrm{p}-\mathrm{p}^{*}\right)$

$\varepsilon_{i}=N\left(0, \sigma^{2}\right) ; \varepsilon_{i}$ independent

$$
A_{i+(j-1) N, k}=w_{i j} \frac{\partial}{\partial p_{k}} M\left(j, p^{e}, t_{i}\right)=-J_{i+(j-1) N, k}
$$

For the least squares solution of this linear problem the following properties can be derived [Seber, 1977]:

$-d p \sim N\left(0, \sigma^{2}\left(A^{+} A\right)^{-1}\right)$

$-s^{2}=\left(1 /\left(N m-N_{p}\right)\right) \sum_{i=1}^{N m} r_{i}^{2}$ is an unbiased estimate of $\sigma^{2}$ with

$$
\left(\mathrm{Nm}-\mathrm{N}_{\mathrm{p}}\right) \mathrm{s}^{2} / \mathrm{o}^{2}-\chi^{2}{ }_{\mathrm{Nm}-\mathrm{N}}
$$


- The $(1-\alpha)$ confidence intervals of the $i-t h$ component $d p_{i}$ of $d p$ is given by

$$
p_{i i}^{e}-S H_{i i} t^{\alpha / 2}<P^{*}{ }_{i}<p_{i i}^{e}+S H_{i i} t^{\alpha / 2}
$$

with $\mathrm{H}_{i \mathrm{i}}=\left(\left(\mathrm{A}^{+} \mathrm{A}\right)^{-1}\right)_{i \mathrm{i}}$

$t^{\alpha / 2}$ - the $\alpha / 2$-percentage point of the student distribution with $\mathrm{Nm}-\mathrm{N}_{\mathrm{p}}$ degrees of freedom

$s=\sqrt{s^{2}}$

outlying_observations

The kind of data to be analysed is most probably contaminated with a not negligible percentage of gross error for which least squares estimation is rather sensitive. In order to avoid the weeding of the data from such outliers this sensitivity was reduced by using for patient data as residuals instead of

$$
r_{i j}^{2}=w_{i j}^{2}\left(C d_{i j}-M(j, p, t)\right)^{2}
$$

the modification cf. [Huber, 1981]

$$
\begin{array}{rlrl}
h r_{i j}^{2} & =r_{i j}^{2} & & ; r_{i j} \leqslant r_{0} \\
& =r_{0}^{2}+2 r_{0} \operatorname{sign}\left(r_{i j}\right) \quad ; r_{i j} \geq r_{0}
\end{array}
$$

in the sum of squared residuals. In view of the anticipated error of about $5 \%$ a conservative value of the scaling factor $r_{0}$ is choosen to be $r_{0}=0.06$, cf. [Andrews, 1972]. This modification reduces the effect of outliers with $\left|r_{i j}\right|>r_{0}$ without discarding them.

Deviations_of the error from_the_assumed_model_(B.11)

The assumptions on the probability distribution of the error terms are difficult to test by analysis of the residuals because of the large number of nuissance parameters $\mathrm{F}_{1}, \ldots, \mathrm{F}_{\mathrm{Ns}}$.

On the other hand relaxing the assumptions on the error by 
introduction of more degrees of freedom in the model, e.g. by only assuming that

$$
\begin{aligned}
& E_{i j} \text { and } \varepsilon_{\ell k} \text { - independent for } i \neq k \\
& \left(w_{i 1} \varepsilon_{i 1}, \ldots, w_{i m} \varepsilon_{i m}\right) \sim \mathbb{N}(0, \Sigma)
\end{aligned}
$$

with unknown covariance matrix $\Sigma$ to be estimated from the data, is not attractive. Firstly because the maximum likelihood estimation of such models generally involves the minimization of the determinant of the residuals as a function of the parameters, cf. [Box, 1965]. A much less tractable problem than the minimization of a sum of squared residuals. Secondly such problems, as is the case with the above example, become unidentifiable if the parametrization of the input function is so flexible that by adjusting the parameters $F_{1}, \ldots, F_{N s}$ the deviation between the model response of one of the enzyme activities and the measured plasma activities can be made equal to zero for all i simultaneously, cf. [Kendal1 (Ch. 29), 1973].

In order to examine the sensitivity of the proposed least squares estimator for deviations in the distribution of the errors data were simulated according to Chapter 3.3 and Appendix B. 4 with various deviating errors.

Errors independent for different sample times and with a normal distribution are generated with a quasi-random number generator i.e.

$$
\left|\begin{array}{l}
E_{11} \\
E_{12}
\end{array}\right| \sim N(0, \Sigma)
$$

$\begin{aligned} & \text { I } \\ & \text { with in case II } \\ & \text { III }\end{aligned}: \Sigma=\left\{\begin{array}{ll}\sigma_{1}^{2} \phi \\ \phi \quad \sigma_{2}^{2}\end{array}\right\} \quad \begin{aligned} & I: 0.05 \\ & \text { II }: \sigma_{1}=0.05 ; \sigma_{2}=0.05 ; \sigma_{2}=0.02 \\ & \text { III: } \sigma_{1}=0.02 ; \sigma_{2}=0.05\end{aligned}$ and

with in case IV $\left.\begin{array}{c}\mathrm{V} \\ \mathrm{V}\end{array} \quad \Sigma=\sigma^{2} \mid \begin{array}{ll}1 & \mathrm{R} \\ \mathrm{R} & 1\end{array}\right\}$

IV: $\sigma=0.05 ; \quad R=0.80$

$\mathrm{V}: \sigma=0.05 ; \quad \mathrm{R}=-0.80$ 
In Table B.2 the mean and standard deviation of the estimated parameters as obtained from 100 different simulated data sets are presented. From these data it is apparent that:

- The least squares estimator is rather robust to this kind of deviations

- The performance of the estimator is especially sensitive to the sampling error contaminating the slowly eliminated enzyme activities.

Table B.2 The effect of deviant ercors upon the performance of the estimator.

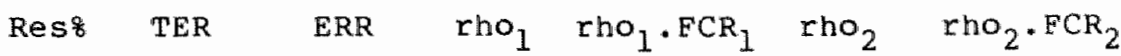

Reference

0.0140 .018

1.00 .015

0.14

0.028

values

I

$$
\bar{x}
$$$$
\bar{x} \quad 5.0 \quad 0.0157
$$

$0.0188 \quad 1.0$

0.0154

0.135

0.0289

SD $0.7 \quad 0.0053$

0.0038

0.0021

0.025

0.0032

II

$\begin{array}{rr}\bar{x} & 4.5 \\ \text { SD } & 0.6\end{array}$

0.0154

0.0187

1. 0

0.0154

0.0136

0.0287

$0.0048 \quad 0.0035$

0.0019

0.023

0.0028

III

$\begin{array}{rr}\overline{\mathrm{x}} & 2.9 \\ \mathrm{SD} & 0.5\end{array}$

0.01520 .0190

1.0

0.0154

0.135

0.0288

$0.0026 \quad 0.0018$

0.0011

0.016

0.0017

IV

$\overline{\mathrm{x}} \quad 4.4$

$\begin{array}{lll}0.0156 & 0.0193 & 1.0\end{array}$

0.0153

0.138

0.0285

SD 0.7

$0.0040 \quad 0.0034$

0.0016

0.019

0.0024

V

$\begin{array}{rrrrc}\bar{X} & 5.2 & 0.0175 & 0.0202 & 1.0 \\ \text { SD } & 0.8 & 0.0075 & 0.0047 & -\end{array}$

0.0162

0.128

0.0297

$0.0027 \quad 0.031$

0.0045 
B4 The calculation of simulated_data

Reference data

The calculation of the plasma response upon the reference input

$$
f(t)=c t^{2} e^{-a t}, c=0.78, a=0.111
$$

depends upon the calculation of the convolution integral

$$
g(t)=\int_{0}^{t} f(\tau) e^{-k(t-\tau)} d \tau=f(t)^{*} e^{-k t}
$$

Laplace transformation of $g(t)$ results in

$$
\bar{g}(s)=\bar{f}(s) \frac{1}{s+k}=\frac{2 C}{(s+a)^{3}(s+k)}
$$

By partial fraction expansion this last formula is reduced to $(k \neq a)$

$$
\bar{g}(s)=\frac{2 c}{(k-a)^{3}}\left(\frac{(k-a)^{2}}{(s+a)^{3}}-\frac{(k-a)}{(s+a)^{2}}+\frac{1}{s+a}-\frac{1}{s+k}\right\}
$$

which corresponds in the time domain to

$$
g(t)=\frac{c}{(k-a)^{3}}\left((k-a)^{2} t^{2} e^{-a t}-2(k-a) t e^{-a t}+2 e^{-a t}-2 e^{-k t}\right)
$$

With given circulatory model parameters $\mathrm{CS}_{j}$, rho ${ }_{j}, \mathrm{FCR}_{j}$, TER and ERR the simulated plasma response is then calculated by (cf. Formula's (3.3) and 3.4)):

$$
\begin{aligned}
& k_{1,2}=\frac{l}{2}\left(\mathrm{ECR}_{\mathrm{j}}+\mathrm{TER}+\mathrm{ERR} \pm \sqrt{\left(\mathrm{FCR}_{\mathrm{j}}+\mathrm{TER}+\mathrm{ERR}\right)^{2}-4 F C R \mathrm{ERRR}}\right) \\
& P_{1}=\frac{k_{1}-E R R}{k_{1}-k_{2}} \quad ; P_{2}=\frac{k_{2}-E R R}{k_{2}-k_{1}} ; C=c_{j} / r h o_{j} \\
& C_{j}(t)=C S_{j}+P_{1} \frac{c_{j}}{\left(k_{1}-a\right)^{3}}\left(\left(k_{1}-a\right)^{2} t^{2} e^{-a t}-2\left(k_{1}-a\right) t e^{-a t}+2 e^{-a t}-2 e^{-k_{1} t}\right) \\
& +\mathbb{P}_{2} \frac{C_{1}}{\left(k_{2}-a\right)^{3}}\left\{\left(k_{2}-a\right)^{2} t^{2} e^{-a t}-2\left(k_{2}-a\right) t e^{-a t}+2 e^{-a t}-2 e^{-k_{2} t}\right\}
\end{aligned}
$$


Analogeously the plasma response for the three compartment mammilary model upon this reference input $f(t)$ is calculated by using Formula (A.16).

Local degradation

In this case, cf. Formula (3.5), the input into plasma is given by

$$
\begin{aligned}
& f(t)=a C\left(\left(t e^{-a t}\right) *\left(e^{-b t}\right)\right) ; \text { with } c=14.1 \\
& a=0.111 \\
& b=a+k_{o i}
\end{aligned}
$$

The plasma concentration in response to this input is represented as a sum of terms

$$
g(t)=f(t) * e^{-k t}
$$

Laplace transformation of this last expression results in

$$
\bar{g}(s)=\frac{a c}{(s+a)^{2}(s+b)(s+k)}
$$

which is reduced by partial fraction expansion to

$$
\begin{array}{r}
\bar{g}(s)=a c\left\{\frac{1}{(b-a)(k-a)} \frac{1}{(s+a)^{2}}+\frac{2 a-b-k}{(b-a)^{2}(k-a)^{2}} \frac{1}{s+a}\right. \\
\left.+\frac{1}{(a-b)^{2}(k-b)} \frac{1}{s+b}+\frac{1}{(a-k)^{2}(b-k)} \frac{1}{s+k}\right\}
\end{array}
$$

which corresponds in the time domain to

$$
g(t)
$$$$
=a c\left(\frac{t e^{-a t}}{(b-a)(k-a)}+\frac{(2 a-b-k) e^{-a t}}{(b-a)^{2}(k-a)^{2}}+\frac{e^{-b t}}{(a-b)^{2}(k-b)}+\frac{e^{-k t}}{(a-k)^{2}(b-k)}\right)
$$ 

CHAPTER 4

ESTIMATION OF THE CIRCULATORY PARAMETERS OE CARDIAC ENZYMES IN PATIENTS WITH AMI

\subsection{Introduction}

In this chapter the simultaneously sampled plasma activity curves of several cardiac enzymes, e.g. CR, CK-MB, HBD and AST as measured in patients with AMI are analysed. The method exposed in the previous chapter is used to estimate the circulatory model parameters of these enzymes in man.

The circulatory parameters of the two-compartment model are identified by simultaneous analysis of HBD plasma activity curves together with CK or AST plasma activities. Next it is verified that the simplified procedures, introduced in section 3.6, are also applicable for the determination of the FCR of rapidiy eliminated enzymes. It is demonstrated that once the circulatory parameters TER, ERR and FCR are determined for the slowly catabolized enzyme HBD there is little benefit in the use of the full size regression problem instead of the simplified method with a fit of only the initial stage of 36 to 48 hours of the plasma activity curves, cf. Section 3.6. This last shorter fit also has the advantage that the perturbation caused by release of liver enzymes is avoided and thus is applicable to ALT plasma activity curves.

Plasma activities of HBD, CK and AST are composed of the activities of two or more isoenzymes. This rises the question whether the estimation of the circulatory parameters by analysis of total activities instead of the separate isoenzyme activities may cause errors. This issue is investigated by estimation of the FCR values of the separate isoenzymes contributing predominantly to the total plasma activity, i.e. $\mathbb{L D H}_{1}$ and $\mathrm{LDH}_{2}$ for $H B D, C K-M M$ for CK and CAST for AST.

A main problem is the validation of the parameter values obtained by the simultaneous analysis of plasma activity curves. The validity of the results depends on the parallel release of 
the enzymes studied. In the prewious chapter it was demonstrated that a simple delay in the release of one of the enzymes will result in a erconeous value of rho, $i . e$. in the estimated value of the ratio of the amounts of enzyme celeased into plasma. Thus a value of tho deviating from the ratio found in heart tissue may in this case be used to detect such a departure.

The most natural cause for non-simultaneous input of enzymes into plasma however is the local degradation of one of the enzymes in the damaged tissue. In section 3.5 it was shown that this kind of departure from parallel release of enzymes does not result in large deviations in the estimated value of rho, whereas especially the estimation of the $\mathbb{E C R}$ of the rapidly eliminated enzyme is severely affected: Inactivation of e.g. the rapidly eliminated enzyme such that only 508 of the enzyme activity released from tissue in the interstitium reaches the plasma would result in an overestimation of the FCR of this enzyme of approximately 100\%, i.e. the true value of FCR is only 50 of the estimated value.

As a possible check against a non-parallel release of enzymes into plasma the fitting of FCR's of three simultaneously sampled enzymes is considered. It is investigated to which extend a disparity in the release of one of the enzymes will be reflected in discrepancies in the estimated values of the FCR's when the FCR's are determined by fitting the model to the three possible combinations of two enzymes.

Another check is offered by the much earlier enzyme release obserwed when patients with AMI are treated with streptokinase. The shorter duration of enzyme release reduces local denaturation which should result in discrepancies between the parameter values determined in the control patients and the parameter values determined in patients treated with streptokinase and in control patients.

The estimation procedure used shows a pronounced sensitivity to sampling error and correspondingly large variations in the estimated values of the circulatory parameters. This renders it impossible to reach conclusions regarding the interindividual variation of the circulatory parameters and rho. Therefore cumu- 
lative release of enzymes is also calculated using fixed mean values of the cicculatory parameters. The ratio of the so calculated cumulative release of different enzymes exhibits a variation due to the neglected interindividual variation in the circulatory parameters and cho. Assuming independence of the various components of this variation one may arrive at an estimation of the interindividual variation in the circulatory parameters. 


\subsection{Methods}

\section{Patients}

In this study plasma enzyme activity curves determined in several groups of patients with acute myocardial infarctions not complicated by cardiogenic shock are analysed. The first group A consists in patients admitted to the coronary care unit of the Academic Hospital Leiden. Clinical details have been reported earlier [Witteveen, 1975]. Blood samples for the determination of $\mathrm{CK}$ and $\mathrm{HBD}$ were obtained immediately after admission to the coronary care unit and then every 4 hours until 48 hours after admission, every 8 hours upto 72 hours, thereafter every 12 hours up to 120 hours and finally once a day for two weeks in 30 patients and for one week in 10 patients. Additional (iso-) enzymes were determined in subgroups of these 40 patients: CK-MB $(n=16)$, LDH-isoenzymes in the ten patients sampled for one week, total AST $(n=20)$ and mitochondrial AST $(n=10)$, GPI $(n=14)$ and ALT $(n=18)$.

A second group of patients, admitted with AMI to the CCU of the Academic Hospital Maastricht, was studied as part of a randomised trial on the effect of intracoronary streptokinase treatment. Patients arriving in the CCU were admitted to the study if: admission to the $\mathrm{CCU}$ occured within four hours after first symptoms, age was below 70 years, pain was still present upon admission and persistent ST shifts larger than $0.2 \mathrm{mV}$ and not reacting upon treatment with nitrobate or beta blockers. Excluded from the study were also: patients earlier exposed to streptokinase treatment, patients subjected earlier to coronary bypass surgery, patients with trauma, e.g. caused by cardiac massage, patients with lowered consciousness precluding informed consent, and patients with an elevated bleeding risk, e.g. because recent surgery. After admission and obtained informed consent the patients were randomly allocated to the treatment (BT, $n=32$ ) or control group $(B C, n=28)$. Part of the patients in the treatment group $(n=19)$ received immediately after admission 100000-500000 U SK iv. Then heart catheterisation was started and after locali- 
sation of the occlusion streptokinase was infused at a rate of $4000 \mathrm{U} / \mathrm{min}$ until $15 \mathrm{~min}$. after recanalisation or otherwise upto a total of $250000 \mathrm{U}$. Patients received additional standard medication.

Activities of $H B D, C K$ and AST were determined in serial plasma samples taken immediately after admission, every 4 hours upto 24 hours thereafter and then every 6 hours for the next 24 hours.

Sample_handiling

Blood samples of 5-10 $\mathrm{mi}$ were obtained generally by using an indwelling intravenous catheter. Clotting was prevented with sodium citrate. Samples were centrifugated immediately for 15 min at $1000 \mathrm{~g}$ in order to remove blood cells. Thereafter, plasma samples were centrifuged a second time for $20 \mathrm{~min}$ at $40.000 \mathrm{~g}$ (first group) or for $15 \mathrm{~min}$ at $1000 \mathrm{~g}$ (SK trial) and stored at $-20^{\circ} \mathrm{C}$ (first group) or $-80^{\circ} \mathrm{C}$ ( $\mathrm{SK}$ trial) until enzyme determinations were performed within 6 weeks after storage.

\section{Enzyme_de_texeminationions}

Activity of alpha-hydroxybutyrate dehydrogenase (HBD) was determined spectrophotometrically at $25^{\circ} \mathrm{C}$ with alpha-ketobutyrate as substrate [Rosalki, 1960] using a test kit (Boehringer, 124818). Lactate dehydrogenase (LDH) activity was assayed spectrophotometrically at $25^{\circ} \mathrm{C}$ with pyruvate as substrate [Wroblewski, 1955] using a testkit (Boehringer, 124923). Isoenzymes of IDH were separated by electrophoresis on agarose films, stained with nitroblue tetrazolium and scanned at 545 m. Creatine kinase (CK) activity was measured at $25^{\circ} \mathrm{C}$ with creatine phosphate as substrate [Rosalki, 1967] using a testkit (Baker 3107 and 3108). Results were converted to equivalent values at $30^{\circ} \mathrm{C}$ by multiplying them with a factor 1.47. The plasma activities of $\mathrm{CK}$ of the patients studied in the $\mathrm{SK}$ trial were assayed by the same method but using a different (N-acetylcysteine activated) testkit (Merck 14109). Isoenzyme separation of muscle CK (CK-MM) and the cardiac isoenzyme (CK-MB) were performed with a column chromatographic method based on stepwise elution of isoenzymes lMercer, 
19751. The actiwty of aspartate aminotransferase (AST) was assayed with aspartate and alpha-ketoglutarate as substrates at $25^{\circ} \mathrm{C}$ [Karmen, 1955] using a testkit (Boehringer 124427). For separation of the AST in cytoplasmic and mitochondrial fraction a chromatographic method was employed [Sampson, 1978]. Plasma enzyme actiwity of ALT was determined at $25^{\circ} \mathrm{C}$ with alpha-ketoglutarate and alanine as substrates [Wroblewski, 1955] using a testkit (Boehringer 124559 ). The activity of GPI was assayed at $25^{\circ} \mathrm{C}$ with (D-) fructose 6-phosphate as a substrate [Bueding, 1955] using a testkit (Sigma $355 \mathrm{~A}, \mathrm{~B}$ ).

Enzyme determinations were checked during the long period in which these data were collected against standards, especially with the transition to a new testkit, and normalized such that the values presented in Table 4.1 are found for the enzyme content of homogenized and sonificated normal human heart tissue.

Table 4.1 Enzyme content of human heart

$\begin{array}{lrrlrr}\text { Enzyme } & \text { MEAN } & \text { CV } & \text { Enzyme } & \text { MEAN } & \text { CV } \\ \text { HBD } & 123 & 9 & \text { LDH } & 155 & 8 \\ \text { CK } & 865 & 10 & \text { LDH }_{1} & 84 & 10 \\ \text { CK-MB } & 132 & 27 & \text { LDH }_{2} & 57 & 9 \\ \text { AST } & 146 & 11 & \text { LDH }_{3} & 12 & 17 \\ \text { MASTI } & 92 & 12 & \text { LDH4 }_{4} & 3 & 33 \\ \text { GPT } & 143 & 10 & \text { LDH }_{5} & 0 & - \\ \text { ALT } & 5 & 37 & & & \end{array}$

Figures indicate mean activities $(U) \pm$ coefficient of variation ( per gram of wet weight as determined in biopsies $(n=8)$ obtained during surgery lVan der Laarse, $1980 \mathrm{~b}]$. Activities were determined at $25^{\circ} \mathrm{C}$ after sonification of samples in buffered saline. 


\section{3 Results}

Analysis_of_CK_and_HBD_plasma_activity_curves

In the 30 patients in group $A$ sampled upto 14 days after admission to the hospital the circulatory parameters as estimated by simultaneous analysis of the plasma activity curves of $\mathrm{CK}$ and HBD are presented in Table 4.2 . As observed earlier with simulated curves, estimation of $\mathbb{F C R}_{C K}$ results in outlying values (and consequently biased estimation of FCR). Therefore we consider henceforth the parameters tho and rho.FCR, cf. Section 3.4.

The mean observed plasma activities of $\mathrm{CK}$ and HBD are shown in Fig. 4.1 together with the (coinciding) mean fit curves. In order to check whether the simpler one-compartmental model, i.e. TER=0 and $E / P=T E R / E R R=0$, allows also an adequate description of the plasma activity curves, the same procedure for the one-compart-

$$
U / 1
$$

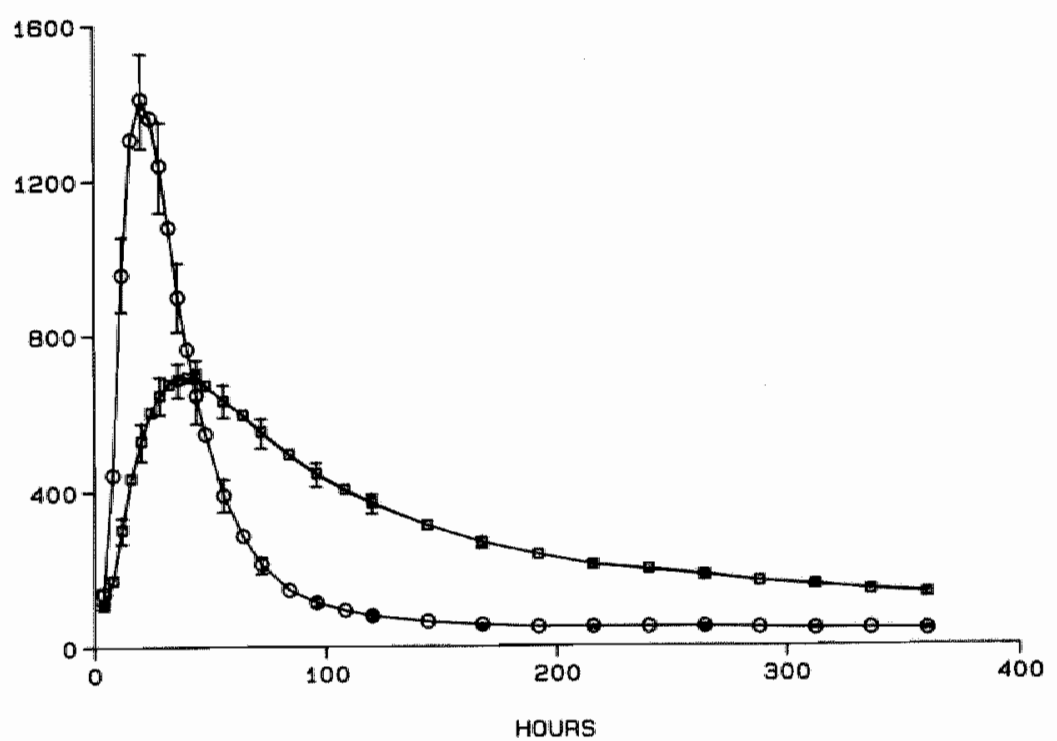

Fig. 4.1 Plasma activities of $C K(O)$ and $H B D$ (D) with best fit approximations. Indicated are the mean and the SEM (bars) of the reaults obtained for 30 patiente with AMI, of. Table 4.2. Solid line indicates the mean best fit approximation. 
Table 4.2 Circulatory parameters estimated by simultaneous analysis of CK and HBD plasma activity curves sampled in 30 patients with AMI.

\begin{tabular}{|c|c|c|c|c|c|c|c|c|c|c|}
\hline \multirow[b]{2}{*}{ patient } & \multirow[b]{2}{*}{ RESSO } & \multirow[b]{2}{*}{$\begin{array}{l}T E R \\
h^{-1}\end{array}$} & \multirow[b]{2}{*}{$\begin{array}{l}\text { ERR } \\
h^{-1}\end{array}$} & & & \multirow[b]{2}{*}{$Q_{96}^{\mathrm{HBD}}$} \\
\hline & & & & $\begin{array}{r}\mathrm{CS} \\
\mathrm{w} / 1\end{array}$ & rho & $\begin{array}{c}\text { rho. FCF } \\
h^{-1}\end{array}$ & $\begin{array}{r}\mathrm{Cs} \\
\mathrm{U} / 1\end{array}$ & rho & $\begin{array}{c}\text { tho. } \mathrm{FCR} \\
\mathrm{h}^{-1}\end{array}$ & \\
\hline 1 & 6.0 & 0.008 & 0.017 & 87 & 1.0 & 0.012 & 52 & 0.14 & 0.021 & 985 \\
\hline 2 & 7.6 & 0.020 & 0.026 & 92 & 1.0 & 0.018 & 51 & 0.13 & 0.031 & 1161 \\
\hline 3 & 7.7 & 0.016 & 0.041 & 79 & 1.0 & 0.016 & 36 & 0.11 & 0.024 & 713 \\
\hline 4 & 4.7 & 0.004 & 0.017 & 103 & 1.0 & 0.012 & 34 & 0.08 & 0.018 & 668 \\
\hline 5 & 5.7 & 0.005 & 0.018 & 72 & 1.0 & 0.012 & 45 & 0.16 & 0.017 & 866 \\
\hline 6 & 4.4 & 0.005 & 0.008 & 82 & 1.0 & 0.015 & 48 & 0.11 & 0.019 & 1010 \\
\hline 7 & 7.2 & 0.013 & 0.022 & 118 & 1.0 & 0.019 & 45 & 0.11 & 0.043 & 1181 \\
\hline$\theta$ & 4.9 & 0.001 & 0.017 & 82 & 1.0 & 0.012 & 35 & 0.15 & 0.032 & 1262 \\
\hline 9 & 6.1 & 0.017 & 0.017 & 106 & 1.0 & 0.022 & 14 & 0.20 & 0.046 & 1795 \\
\hline 10 & 6.4 & 0.006 & 0.017 & 83 & 1.0 & 0.012 & 26 & 0.21 & 0.028 & 1195 \\
\hline 11 & 6.6 & 0.015 & 0.022 & 106 & 1.0 & 0.016 & 35 & 0.06 & 0.021 & 629 \\
\hline 12 & 4.0 & 0.009 & 0.018 & 87 & 1.0 & 0.012 & 42 & 0.23 & 0.028 & 1217 \\
\hline 13 & 5.1 & 0.027 & 0.020 & 75 & 1.0 & 0.011 & 49 & 0.10 & 0.023 & 534 \\
\hline 14 & 7.1 & 0.013 & 0.012 & 65 & 1.0 & 0.011 & 33 & 0.09 & 0.020 & 1510 \\
\hline 15 & 9.2 & 0.019 & 0.016 & 68 & 1.0 & 0.008 & 33 & 0.06 & 0.024 & 490 \\
\hline 16 & 6.6 & 0.029 & 0.055 & 144 & 1.0 & 0.030 & 30 & 0.08 & 0.030 & 660 \\
\hline 17 & 3.7 & 0.006 & 0.009 & 79 & 1.0 & 0.016 & 44 & 0.12 & 0.029 & 1453 \\
\hline $1 \mathrm{~B}$ & 3.6 & 0.097 & 0.014 & 84 & 1.0 & 0.013 & 28 & 0.08 & 0.019 & 729 \\
\hline 19 & 5.3 & 0.014 & 0.023 & 140 & 1.0 & 0.019 & 87 & 0.04 & 0.019 & 1850 \\
\hline 20 & 5.8 & 0.013 & 0.025 & 128 & 1.0 & 0.019 & 52 & 0.09 & 0.024 & 2182 \\
\hline 21 & 9.1 & 0.013 & 0.021 & 91 & 1.0 & 0.019 & 36 & 0.19 & 0.026 & 1315 \\
\hline 22 & 5.5 & 0.021 & 0.035 & 88 & 1.0 & 0.020 & 67 & 0.07 & 0.022 & 1622 \\
\hline 23 & 7.4 & 0.013 & 0.005 & 146 & 1.0 & 0.014 & 47 & 0.10 & 0.020 & 946 \\
\hline 24 & 6.2 & 0.011 & 0.012 & 109 & 1.0 & 0.016 & 54 & 0.09 & 0.019 & 552 \\
\hline 25 & 7.4 & 0.016 & 0.047 & 116 & 1.0 & 0.016 & 57 & 0.18 & 0.017 & 1985 \\
\hline 26 & 4.8 & 0.015 & 0.015 & 86 & 1.0 & 0.014 & 22 & 0.25 & 0.034 & 2395 \\
\hline 27 & 5.2 & 0.011 & 0.005 & 65 & 1.0 & 0.016 & 46 & 0.15 & 0.021 & 1336 \\
\hline 28 & 4.9 & 0.034 & 0.042 & 89 & 1.0 & 0.018 & 41 & 0.07 & 0.021 & 1082 \\
\hline 29 & 3.8 & 0.025 & 0.027 & 76 & 1.0 & 0.010 & 76 & 0.11 & 0.018 & 1450 \\
\hline 30 & 9.5 & 0.003 & 0.011 & 94 & 1.0 & 0.013 & 94 & 0.17 & 0.027 & 1229 \\
\hline mean & 6.0 & 0.014 & 0.021 & 95 & 1.0 & 0.015 & 43 & 0.12 & 0.025 & 1.203 \\
\hline $\mathrm{SD}$ & 1.6 & 0.008 & 0.012 & 23 & - & 0.004 & 14 & 0.06 & 0.007 & 495 \\
\hline SEM & 0.3 & 0.001 & 0.002 & 4 & - & 0.001 & 3 & 0.01 & 0.001 & 90 \\
\hline
\end{tabular}


ment model was applied to the data. With this procedure the following values (meantSEM) were found for the circulatory parameters of the one-compartment model:

$$
\begin{array}{ll}
\text { ChOHBD }_{\mathrm{HBK}} & =0.151 \pm 0.009 \\
\mathrm{ECR}_{\mathrm{HBD}} & =0.0134 \pm 0.007 \\
\text { PCR }_{\mathrm{CK}} & =0.124 \pm 0.016 \\
\text { Res: }^{5} & =7.7 \% \pm 0.3
\end{array}
$$

These values deviate significantly from the corresponding values of the circulatory parameters found with the two-compartment analysis. The inadequacy of this model is apparent from the systematic deviations between data and fit curves shown in $F i g$. 4.2. This in contrast to the situation with the two-compartment fit were the mean residual deviation between data and fit is not different from zero.

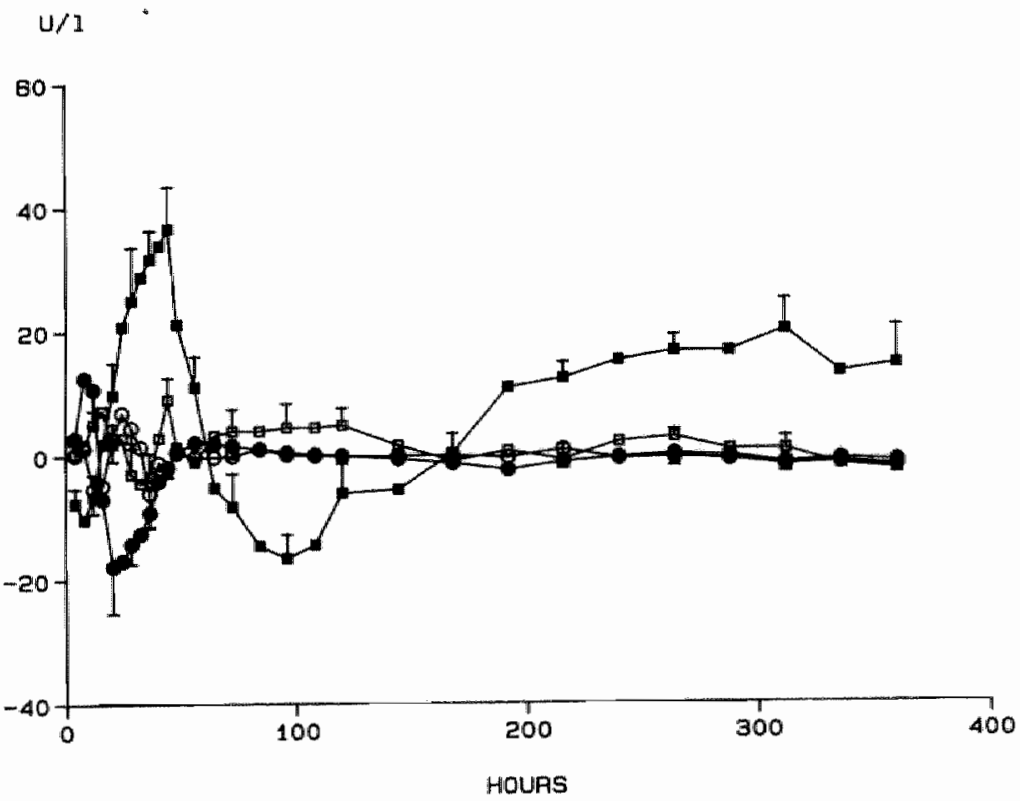

Fig. 4.2 Residual deviation between data and fit for the one-compartment model (CK: ; $H B D: \mathbf{n})$ and for the two-compartment model (CK: 0 ; $H B D: \square$ ) cf. Fig. 4.1. 

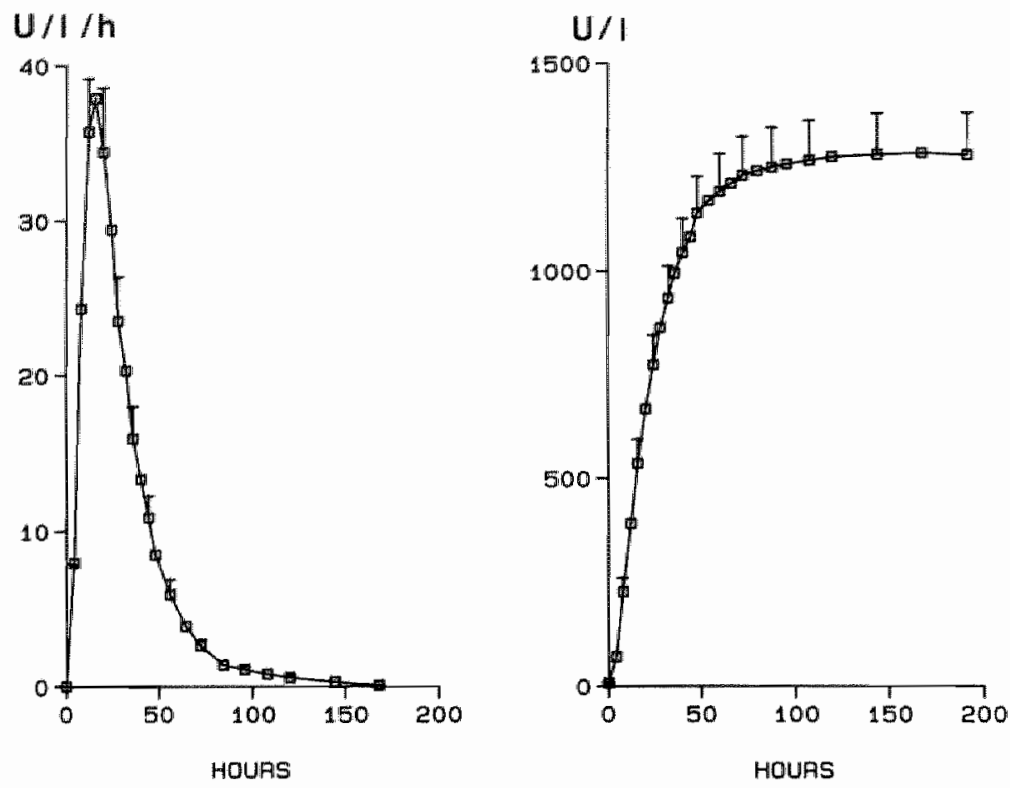

Fig. 4.3 Mean input of HBD into plasma as obtained for the patients from Table 4.2. Left ponel: mean input function f(tl) Right panel: the mean cumulative release.

The mean estimated input into plasma of HBD, expressed in $U / 1 / h$, $\left(\mathrm{f}_{\mathrm{HBD}}\right.$ ) and its integral representing the cumulative release upto time $t$ are shown in Fig. 4.3 .

S1 1 mult_aneous_analysis_of_CK__HBD_and_AST_Plasma_activitities

In a subgroup of 20 patients of group $A$ also AST plasma activities were determined. Simultaneous analysis of the $C K, H B D$ and AST plasma activity curves results in estimated values of the circulatory parameters as presented in Table 4.3. Apparently the inclusion of AST in the fit does not affect the values of the circulatory parameters of $\mathrm{CK}$ and $\mathrm{HBD}$, cf Table 4.2.

The ratios of the released quantities of the enzymes, i.e. $\mathrm{rho}_{\mathrm{HBD} / \mathrm{CK}}$ and $\mathrm{rho}_{\mathrm{HBD} / \mathrm{AST}}$ do not differ from the ratio $\mathrm{HBD} / \mathrm{CK}$ and HBD/CAST found in heart tissue, cf. Table 4.1. Therefore it seems appropriate to express cumulative release of enzymes in gram 
Table 4.3 Circulatory parameters estimated by simultaneous analysis of CK. AST and ABD plasma activity curves sampled in 20 patients with AMI.

\begin{tabular}{|c|c|c|c|c|c|c|c|c|c|}
\hline Res? & \multirow[b]{2}{*}{ TER } & \multirow[b]{2}{*}{ ERR } & \multicolumn{2}{|r|}{$\mathrm{HBD}$} & \multicolumn{3}{|c|}{$\mathrm{CK}$} & \multicolumn{2}{|r|}{$\mathrm{AST}$} \\
\hline pat. & & & cs & rho rho. FCR & $\mathrm{CS}$ & rho & rho. FCR & $\operatorname{cs}$ & rho tho. $\mathbb{F C R}$ \\
\hline & $h^{-}$ & $\mathrm{h}^{-}$ & $0 / 1$ & $h^{-1}$ & $0 / 1$ & & $n^{-1}$ & $u / 1$ & $h^{-1}$ \\
\hline
\end{tabular}

$\begin{array}{rrrrrrrrrrrrr}11 & 5.5 & 0.009 & 0.016 & 106 & 1.1 & 0.014 & 35 & 0.07 & 0.017 & 12 & 1.6 & 0.16 \\ 12 & 4.3 & 0.011 & 0.020 & 92 & 1.0 & 0.013 & 42 & 0.21 & 0.030 & 9 & 2.3 & 0.17 \\ 13 & 4.9 & 0.023 & 0.018 & 75 & 1.0 & 0.011 & 49 & 0.10 & 0.022 & 6 & 2.4 & 0.22 \\ 14 & 9.0 & 0.020 & 0.014 & 67 & 1.0 & 0.013 & 33 & 0.08 & 0.022 & 16 & 2.3 & 0.19 \\ 15 & 7.4 & 0.013 & 0.013 & 62 & 1.0 & 0.006 & 33 & 0.09 & 0.020 & 8 & 1.6 & 0.14 \\ 16 & 5.2 & 0.044 & 0.059 & 138 & 1.0 & 0.032 & 30 & 0.08 & 0.032 & 5 & 1.5 & 0.25 \\ 17 & 5.7 & 0.008 & 0.014 & 83 & 1.0 & 0.018 & 47 & 0.10 & 0.032 & 12 & 2.0 & 0.15 \\ 18 & 3.3 & 0.016 & 0.014 & 83 & 1.0 & 0.013 & 28 & 0.08 & 0.018 & 6 & 2.1 & 0.20 \\ 19 & 5.8 & 0.007 & 0.005 & 97 & 1.0 & 0.010 & 87 & 0.10 & 0.015 & 5 & 1.9 & 0.20 \\ 20 & 6.4 & 0.010 & 0.022 & 125 & 1.0 & 0.017 & 52 & 0.11 & 0.022 & 9 & 3.6 & 0.24 \\ 21 & 7.4 & 0.012 & 0.020 & 94 & 1.0 & 0.019 & 36 & 0.18 & 0.026 & 11 & 2.5 & 0.21 \\ 22 & 8.4 & 0.056 & 0.045 & 91 & 1.0 & 0.030 & 67 & 0.03 & 0.030 & 10 & 2.1 & 0.41 \\ 23 & 7.5 & 0.012 & 0.007 & 145 & 1.0 & 0.015 & 47 & 0.12 & 0.020 & 7 & 1.5 & 0.18 \\ 24 & 4.6 & 0.011 & 0.012 & 110 & 1.0 & 0.017 & 54 & 0.08 & 0.020 & 9 & 2.2 & 0.23 \\ 25 & 7.6 & 0.014 & 0.043 & 118 & 1.0 & 0.015 & 57 & 0.19 & 0.017 & 8 & 2.7 & 0.17 \\ 26 & 5.8 & 0.014 & 0.013 & 84 & 1.0 & 0.014 & 22 & 0.23 & 0.034 & 8 & 2.6 & 0.22 \\ 27 & 5.6 & 0.011 & 0.005 & 66 & 1.0 & 0.016 & 47 & 0.16 & 0.020 & 9 & 3.3 & 0.35 \\ 28 & 5.0 & 0.019 & 0.030 & 87 & 1.0 & 0.014 & 41 & 0.09 & 0.018 & 6 & 2.3 & 0.19 \\ 29 & 3.7 & 0.023 & 0.026 & 76 & 1.0 & 0.010 & 54 & 0.12 & 0.017 & 9 & 2.6 & 0.21 \\ 30 & 8.1 & 0.004 & 0.016 & 78 & 1.0 & 0.012 & 36 & 0.22 & 0.027 & 7 & 3.2 & 0.13 \\ & & & & & & & & & & & & \\ \text { mean } & 6.0 & 0.017 & 0.021 & 93 & 1.0 & 0.015 & 43 & 0.12 & 0.023 & 8 & 2.3 & 0.21 \\ \text { SD } & 1.6 & 0.012 & 0.014 & 21 & - & 0.006 & 16 & 0.06 & 0.006 & 3 & 0.6 & 0.07 \\ \text { SEM } & 0.4 & 0.003 & 0.003 & 5 & - & 0.001 & 4 & 0.01 & 0.001 & 1 & 0.1 & 0.02\end{array}$

equivalents per litre of plasma (q-eq/1), i.e. total cumulative release expressed in units per litre (U/1) is divided by the heart tissue content expressed in $\mathrm{U} / \mathrm{g}$.

\section{Simplifífied_estimation procedures}

In Section 3.6 two simplified procedures for the estimation of FCR were proposed: The results of these methods presented in Table 4.4 conform to the results obtained in section 3.6 with simulated data, i.e. estimates of rho and rho.FCR obtained with the simplified methods are in agreement to the results of the general procedure cf. Table 4.3. The simplified method (I) allows also the identification of $F C R_{C K}$ and $F C R_{A S T}$ without reference to 
Table 4.4 simplified estimation procedures applied to the analysis of CK. HBD and AST plasma activities after AMI.

\begin{tabular}{|c|c|c|c|c|c|c|c|c|}
\hline \multirow[b]{2}{*}{$\operatorname{Res}$} & \multirow[b]{2}{*}{$\begin{array}{l}\mathrm{TE} \\
\mathrm{h}^{-1}\end{array}$} & \multirow[b]{2}{*}{$\begin{array}{l}\text { ERR } \\
\mathrm{h}^{-1}\end{array}$} & \multicolumn{2}{|c|}{$\mathrm{HBD}$} & \multicolumn{2}{|c|}{ CK } & \multicolumn{2}{|c|}{ AST } \\
\hline & & & rho & $\begin{array}{c}\text { rho. FCR } \\
n^{-1}\end{array}$ & rho & $\begin{array}{c}\text { xho } \cdot \mathrm{PCR} \\
\mathrm{n}^{-1}\end{array}$ & rho & $\begin{array}{c}\text { rho. FCR } \\
h-4\end{array}$ \\
\hline
\end{tabular}

(I) mean $\begin{array}{cccccccccc} & 5.3 & 0.0140 & 0.021 & 1.0 & 0.0156 & 0.126 & 0.0224 & 2.24 & 0.206 \\ \mathrm{SD} & 1.2 & - & - & - & 0.0053 & 0.044 & 0.0060 & 0.55 & 0.065 \\ \mathrm{SEM} & 0.3 & - & - & - & 0.0011 & 0.010 & 0.0013 & 0.12 & 0.015\end{array}$

$\begin{array}{lccccccccc}\text { (II) mean } & 5.1 & 0.0140 & 0.021 & 1.0 & 0.0150 & 0.114 & 0.0223 & 2.19 & 0.204 \\ \text { SD } & 1.5 & - & - & - & - & 0.051 & 0.0053 & 0.58 & 0.047 \\ \text { SEM } & 0.3 & - & - & - & - & 0.011 & 0.0012 & 0.13 & 0.010\end{array}$

(I) Analysils of the data using fixed mean values of TER and ERR and fitting the model to data sampled upto 96 hours.

(II) Analysis of the data with HBD as a reference enzyme with fixed mean values supplied for FCR $\mathrm{HBD}$ " TER and ERR and fitting the model to data sampled upto 48 hours.

HBD data lalthough the mean values of TER and ERR used in thi simplified procedure of course were determined by a $f i t$ of $H B$ and CK plasma curves). The results presented in Table 4.5 indi cate that identical values of $F_{C K}$, AST or HBD are found in dependently of the combination of these enzymes used in th estimation procedure.

The effect of local degradation upon the estimation procedur applied to the three possible combinations of plasma activit curves is assessed by application of the identical estimatio procedure to simulated data constructed conform the model o local degradation exposed in Section 3.5. Hereto the example i: considered that CK suffers from local degradation with the effect that only 508 of $C K$ released from the tissue reaches the circulation, while local imactivations is absent for AST and HBD. Ir that case the value of FCR ${ }_{C K}$ estimated before, cf. Table 4.2-4. 
Table 4.5 Application of the simplified method (I) to the combinations $\mathrm{CK}$ $x$ HBD, CK $\times$ AST and HBD $\times$ AST separately.

Patiencs:

\begin{tabular}{|c|c|c|c|c|c|}
\hline & & & $\mathrm{HBD}$ & CKR & $\mathrm{Ast}$ \\
\hline Res: & $\begin{array}{l}\text { TER } \\
h^{-1}\end{array}$ & $\begin{array}{l}\mathbb{E R R} \\
\mathrm{h}^{-1}\end{array}$ & $\begin{array}{c}\text { tho rho. ECR } \\
h^{-1}\end{array}$ & $\begin{array}{c}\text { tho } \mathrm{FCR} \\
\mathrm{h}^{-1}\end{array}$ & $\begin{array}{c}\text { rho tho } \mathrm{FCR}^{-1} \\
\mathrm{~h}^{-1}\end{array}$ \\
\hline
\end{tabular}

mean

$\begin{array}{lllll}5.3 & 0.014 & 0.021 & 1.0 & 0.0156\end{array}$

$0.116 \quad 0.0224$

2.24

0.206

SD

$1.2-$

$-\quad-0.0053$

0.0440 .0060

0.550 .065

mean

$\begin{array}{lllll}5.0 & 0.014 & 0.021 & 1.0 & 0.0167\end{array}$

$0.110 \quad 0.0227$

SD

$1.8-$

$-\quad-0.0065$

$0.056 \quad 0.0052$

mean

$5.20 .014 \quad 0.021-$

$-$

$0.125 \quad 0.0243$

2.40

0.207

$1.9=$

0.0420 .0106

0.071

mean

$\begin{array}{cccccccccc}4.9 & 0.014 & 0.021 & 1.0 & 0.0160 & - & - & 2.31 & 0.212 \\ 2.4 & - & - & - & 0.0084 & - & - & - & 0.63 & 0.068\end{array}$

Simulations:

Reference

$\begin{array}{llll}0.014 & 0.021 & 1.0 & 0.0150\end{array}$

$0.115 \quad 0.0115$

2.20

0.210

values

mean

$\begin{array}{llllll}4.8 & 0.014 & 0.021 & 1.0 & 0.0169\end{array}$

$0.108 \quad 0.0245$

2.16

0.221

$\mathrm{SD}$

0.7

$-\quad-0.0023$

$0.014 \quad 0.0016$

0.14

0.095

mean

5.10 .014

0.021

$1.0 \quad 0.0166$

$0.110 \quad 0.0246$

$\mathrm{SD}$

1.1 -

- $\quad-0.0024$

$\begin{array}{lll}0.014 & 0.0017\end{array}$

mean

$4.4 \quad 0.01$

$0.021 \quad 1.0$

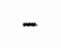

$0.104 \quad 0.0274$

2.20

0.245

SD

0.9

$-$

$0.017 \quad 0.0053$

0.046

mean

$\begin{array}{lllll}4.6 & 0.014 & 0.021 & 1.0 & 0.0155\end{array}$

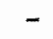

$-$

2.23

0.212

SD

$0.9-\quad-0.0035$

$-$

0.20

0.025

Figures indicate mean and standard deviation of estimated parameters; patients: $n=20$; simulations: $n=10$. For construction of simulated data sets: see text. 
would represent an overestimation of approximately $100 \%$. Of the true value of FCR. Hence in the simulations it was assumed that the inactivation of $\mathrm{CK}$ amounts to $50 \%$ and that the rho. FCR ${ }_{C K}$ equals 0.0115 . Simulated data were contaminated with $5 \%$ random error. From the results shown in Table 4.5 it follows that extensive local inactivation results in discrepancies in the estimated parameter values.

Determination_of_ECR_of_various_lisolenzymes

The values of FCR of $H B D, L D H_{1}, L_{D H}$ are determined in the subgroup of 10 patients of group $A$ by simultaneous analysis with the simplified method I of the plasma activity curves of these enzymes together with $\mathrm{CK}$. The results of this analysis are summarized in Table 4.6 .

Table 4.6 The estimated values of FCR of cardiac (iso-) enzymes.

ENZYME $\begin{array}{cc}h^{-1} & \text { number of } \\ & \end{array}$

$\begin{array}{llcl}\text { HBD } & 0.0150 \pm 0.0008 & 30 & \text { Table } 4.2 \\ \text { LDH }_{1} & 0.0143 \pm 0.0013 & 10 & \\ \text { LDH }_{2} & 0.0154 \pm 0.0012 & 12 & \\ \text { CX } & 0.204 \pm 0.028 & 30 & \text { Table } 4.2 \\ \text { CK-MM } & 0.209 \pm 0.034 & 16 & \\ \text { CK-MB } & 0.329 \pm 0.119 & 16 & \text { Table 4.3 } \\ \text { AST } & 0.0913 \pm 0.0117 & 20 & \\ \text { CAST } & 0.0901 \pm 0.0173 & 10 & \\ & 0.249 \pm 0.043 & 14 & \\ \text { GPI } & 0.0376 \pm 0.0049 & 18 & \end{array}$

Values of $\mathrm{FCR}$ are calculated as $\mathrm{FCR}=$ rho. $\mathrm{FCR} / \mathrm{rho}$. 
The values of ECR for the enzymes ALT, GPI, CK-MM and CAST as presened in Table 4.6 are determined by simultaneous analysis of the plasma activity curves of each of these enzymes with the plasma activity curve of the reference enzyme HBD, i.e. by application of the simplified estimation procedure II upon various subgroups of group A.

\section{Estimation_of FCR_of_CK_and_AST_in_SK-t_egted_patients}

Figure 4.4 shows the input into plasma of HBD and CK, calculated using the fixed mean values of the circulatory parameters in the control patients (group BC) and in the SK-treated patients (group BT). Apparently the SK-treatment results in a much earlier

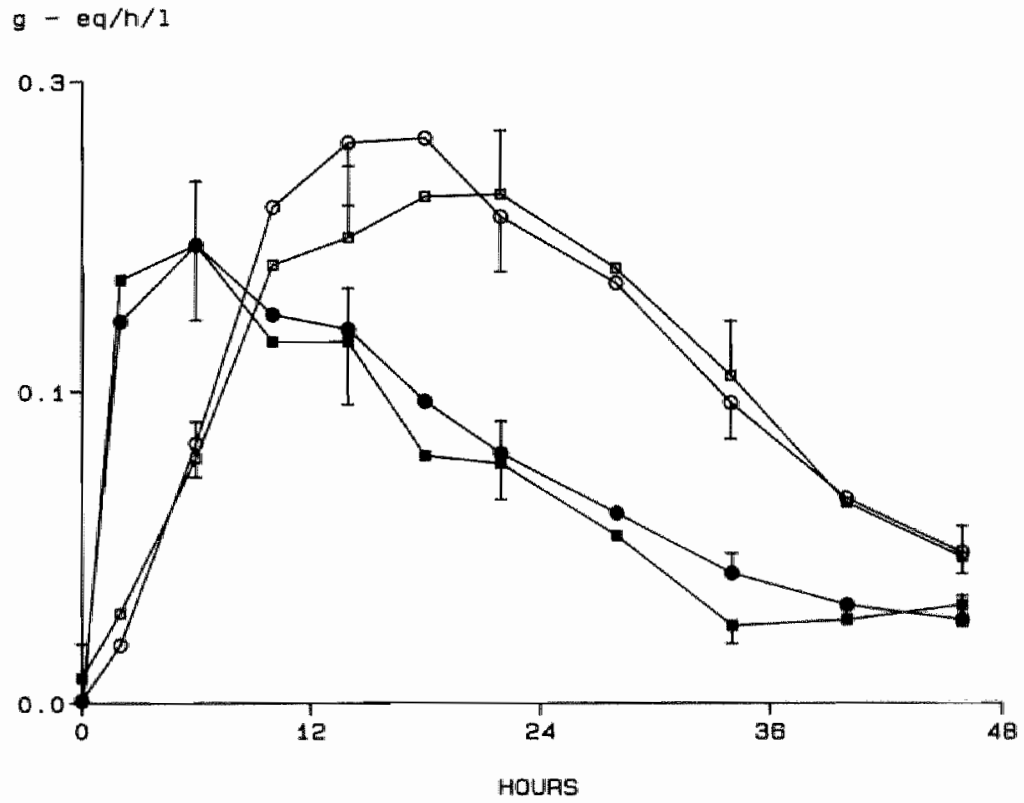

Fig. 4.4 The mean input of $f(t)$ CK $(0,0)$ and of $H B D(\square, 0)$ into plaama expresed in $g-e q / 2 / h$ by dividing the calculated input $f(t)(U / 2 / h)$ by the heart tiaaue enayme content $(U / g)$ (of. Table 4.1). Open aymbota: The mean input in 28 control patients. Closed aymbota: The mean input as obtained in 32 streptokinase treated patients. 
Table 4.7 Estimation of FCR sK-treated patients.

Rest rho rho.FCR $\frac{\text { AST }}{\text { rho rho.FCR }}$

$\begin{array}{lllllll}\text { controls } & \text { mean } & 5.6 & 0.115 & 0.0244 & 1.94 & 0.172 \\ (\mathrm{n}=28) & \text { SEM } & 0.4 & 0.011 & 0.0013 & 0.11 & 0.008 \\ & & & & & & \\ \text { SK treated mean } & 6.5 & 0.123 & 0.0235 & 1.68 & 0.157 \\ \text { patients } & \text { SEM } & 0.5 & 0.013 & 0.0013 & 0.11 & 0.009 \\ (\mathrm{n}=30) & & & & & & \end{array}$

Walues of ECR are calculated as FCR $=$ rho. FCR/ cho

In the controls: $\quad F_{C K}=0.21 \pm 0.03 \quad F_{C R} R_{A S T}=0.089 \pm 0.009$

In the treated patients: $\mathrm{FCR}_{\mathrm{CK}}=0.19 \pm 0.03 \mathrm{FCR}_{\mathrm{AST}}=0.094 \pm 0.011$
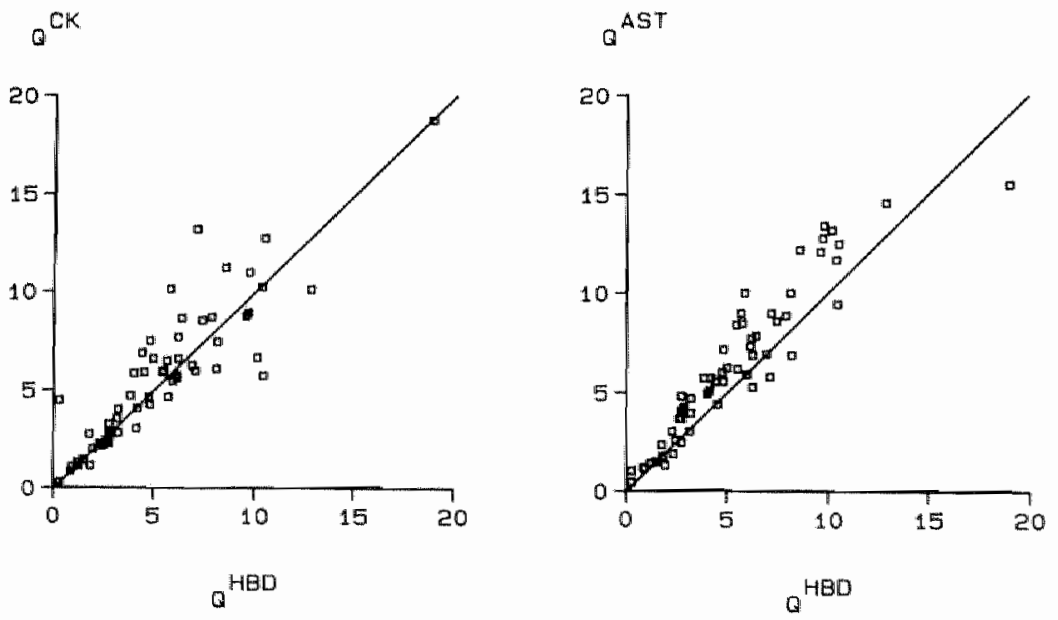

Fig. 4.5 The correiation between the cumulative retease up to 48 houms of $\mathrm{CK}$ and HBD, left panel and of AST and HBD in the patiente of group $B$. The atculated cumulative release $(U / l)$ is expressed in $(g-e q / l)$ by division by heart tissue enzyme content (U/g) (of. Table 4.1 ). 
release of enzymes, without changing the proportion of released $\mathrm{CK}$ and HBD, see also Table 4.8. This indicates already that the $F C R_{C K}$ in SK-treated patients is not different from the value of FCR in control patients. This was checked by application of the simplified procedure II upon the simultaneously samples plasma activity curves of CK, HBD and AST, see Table 4.7.

\section{Correlation_of_enzyme_relétease}

Data on control patients $(n=28)$ and sk-treated patients $(n=32)$ were pooled, because no differences between these groups were detected with respect of the proportion, of release of CK, AST and HBD. Figure 4.5 shows the relation between CK, $H B D$ and $A S T$ cumulative release upto 48 hours in these patients of group $B$. Correlation coefficients and mean ratios of released quantities are presented in Table 4.8 .

Table 4.8 Relation between of the guantities of the enzymes AST, CK and HBD released during the first 48 hours after AMI.

\begin{tabular}{|c|c|c|}
\hline & $Q^{\mathrm{AST}} / \mathrm{Q}^{\mathrm{HBD}} \pm \mathrm{CV}$ & $Q^{\mathrm{CK}} / \mathrm{Q}^{\mathrm{HBD}} \pm \mathrm{CV}$ \\
\hline $\begin{array}{l}\text { Control patients } \\
(n=28)\end{array}$ & $1.16 \pm 208$ & $1.09 \pm 25$ \\
\hline $\begin{array}{l}\text { sk-treated patients } \\
(n=32)\end{array}$ & $1.26 \pm 208$ & $1.04 \pm 238$ \\
\hline $\begin{array}{l}\text { all patients } \\
(n=60)\end{array}$ & $1.22 \pm 208$ & $1.06 \pm 248$ \\
\hline $\begin{array}{l}\text { Correlation } \\
\text { coefficients : }\end{array}$ & AST $x$ HBD & $\mathrm{CK} \times \mathrm{HBD}$ \\
\hline control patients & $R=0.90$ & $\mathrm{R}=0.88$ \\
\hline$(n=28)$ & $Q^{\mathrm{AST}}=1.4+0.93 \mathrm{Q}^{\mathrm{HBD}}$ & $Q^{C K}=1.17+0.86 Q^{\mathrm{HBD}}$ \\
\hline SK-treated patients & $R=0.96$ & $\mathbb{R}=0.87$ \\
\hline$(n=32)$ & $\mathrm{Q}^{\mathrm{AST}}=0.4+1.15 \mathrm{Q}^{\mathrm{HBD}}$ & $Q^{C K}=0.6+0.94 Q^{\mathrm{HBD}}$ \\
\hline al1 patients & $R=0.93$ & $R=0.88$ \\
\hline$(n=60)$ & $Q^{A S T}=0.9+1.02 Q^{\mathrm{HBD}}$ & $Q^{C K}=0.8+0.90 Q^{\mathrm{HBD}}$ \\
\hline
\end{tabular}

Cumulative release of enzyme was expressed in (g-eg/1) by using the values of heart tissue enzyme content given in Tablie 4.1. 
4. 4 Discussion

The assumptions used in the mult t_enzyme_analysis

The two-compartmental multi-enzyme analysis procedure for the estimation of the circulatory parameters is based upon a number of assumptions:

-a- Input of enzyme occurs only in plasma

-b- The exchange of enzyme between plasma and the extrawascular pool may be described by the two-compartment madel, $i . e$. the extravascular pool is homogeneous.

-c- The exchange rates between plasma and extravascular pool of all enzymes included in the analysis is identical. -d-After AMI the release of cardiac enzymes is simultaneous.

The first assumption, already discussed in Chapter 2.6, is based upon Eindings in experimental infarctions in the dog, where it was observed that more than $80 \%$ of the enzymes enters the cicculation directly while the remainder is transported by lymph with a relatively short delay of less then 20 minutes.

The second assumption, i.e. the adoption of the two-compartment model for the dynamics of plasma levels of proteins, presents a simplification. However as discussed in Chapter 2.4, the dynamics of the plasma levels after an intraveneous bolus injection conforms closely to the bi-exponential impulse response of the two-compartment model for most plasma proteins studied, with alloumin as a notable exception exhibiting a tri-exponential plasma disappearance. The plasma decay curves of ALT after an intraveneous bolus injection in the dog could also adequately be described by the two-compartment model [Willems, 1982]. Division of the extravascular pool into two pools with different exchange rates has only limited effects on the values of the estimated parameters as was demonstated by using simulated data, cf. Table 3.7 .

The third assumption, i.e. that the behaviour of enzymes in the circulation does not differ with respect to the transcapillary escape rate or the extravascular return rate, is plausible in view of the experiments on isolated organs summarized in Fig. 
2.2. The values of the exchange cates TER and ERR between the plasma and extravascular pools, as obtained from intraveneous injection of radiolabeled plasma proteins show considerable differences both between different preparations of the same protein and between different proteins. The above mentioned variations in the reported values do not allow definitive conclusions on this issue.

In situations as considered in this chapter only one of the enzymes, HBD, is catabolized at such a slow rate that it is comparable to the rate of extravasation while the cemaining enzymes are eliminated at least 6 times more rapidly. Eor such situations it was shown by using simulations, cf. Table 3.8 , that the estimated values of TER and ERR represent essentially a compromise between the various TER and ERR values, while differences in the values of TER and ERR of the enzymes analysed cause a moderate bias in the estimated circulatory parameters.

The last assumption of parallel release of the enzymes after AMI is the most critical one. In the section 3.5, it was already demonstrated for two-examples (1) delayed release of one of the enzymes and (2) local denaturation of one of the enzymes before release into the plasma, that deviations from this simultaneous release may cause a considerable bias in the estimated values of the circulatory parameters, while the quality of the fit measured by Res' is hardly affected.

The occurrence of delayed release of one of the enzymes compared to the other results in a large bias in the estimated value of tho, ci. Table 3.10. This kind of non-simultaneous release would thus result in gross deviations of the estimated rho from the ratio of the heart content of the corcesponding enzymes. Such deviations were not found, cf. Table 4.2-3.

A similar check upon the occurrence of local denaturation is regrettably lacking. The effect of this kind of deviation from the model is mainly reflected by a biased estimation of the FCR of the rapidly eliminated enzyme compensating for the local denaturation, cE. Table. 3.11.

Two circumstantial arguments plead against the local degradation. Eirstly simultaneous analysis of the time-activity curves 
of SK-treated patients results in values of ECR CK $_{\text {and }}$ FCR $_{\text {AST }}$ not different from the values found in control patients this in spite of the much earlier release of the enzymes in the treated group, see Fig. 4.4. Aso quantificated cumulative release of the enzymes CK, HBD and AST which fixed mean values of FCR, TER and ERR has the same proportions in both groups. This in contrast to several reports on the serum entrance ratios of $C K$ measured im experimental studies in the dog. In this model it was observed that the serum entrance ratio is higher in scattered infarctions [Cairns, 1978; Swain, 1980] compared to massive infarctions and is also higher after reperfusion [Vatner, 1978].

secondly the variable recovery found in these studies is not compatible with the moderate variation, $C V 24 \%$, observed for the ratio of total quantities of calculated released $C K$ and HBD (quantified with fixed mean values of FCR).

The direct check of pairwise analysis of the several different combinations of $C K, H B D$ and $A S T$ plasma activity curves does not result in discrepancies in the estimated parameter values. Accoraing to the simulations presented in Table 4.5 such discrepancies would result in the presence of a massive local inactivation. However with the large variation found in the estimation and the relatively small dilscrepancies predicted by the simulations, this test is disappointingly insensitiwe.

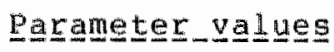

Experimental studies in man addressed to the determination of circulatory model parameters of tissue enzymes by means of intraveneous injections are lacking with as a single exception a study with alkaline phosphatase, cf. Section 2.5. Therefore it is impossible to compare our results directly with other observations.

With respect to the extravasation rate TER and the size of the extravascular pool, i.e. $T E R / E R R=E / P$, there are several reasons, cf. Section 2.2 , to assume that the values of these parameters do not depend critically upon the specific protein in study. Therefore it seems reasonable to compare our estimated 
values to the values obtained for IgG, one of the best studied plasma proteins with a molecular weight close to HBD, cf. Table $2.3:$

IgG

TER

$\mathrm{E} / \mathrm{P}$

$0.011-0.016$

HBD

0.014

$0.85-0.98$

0.68

clearly there exists a good agreement between these values.

As the fractional catabolic rates depend critically upon the specific protein and species in study, such comparisons are not feasible for ECR. Only two reports on the determination of FCR by the intraveneous infusion of cardiac enzymes in man are currently known to us. The values $\mathrm{ECR}_{\mathrm{CK}}=0.16 \mathrm{~h}^{-1}, \mathrm{FCR}_{\mathrm{HBP}}=0.015 \mathrm{~h}^{-1}$ $(n=1)$, [Dawson, 1969] and $F_{C R}=0.12 \pm 0.015 \mathrm{~h}^{-1}$ (mean \pm SEM, $\mathrm{n}=6$ ) [Tommasini, 1979] are in a reasonable agreement with our results.

All estimations of the circulatory parameters show a huge variation which is larger than the variation observed in application of the estimation procedure to simulated data contaminated with a comparable level of (independent) error. Especially the standard deviation of about 408 in the estimated values of rho $\mathrm{HBD} / \mathrm{CK}$ is much larger than the standard deviation found with simulated data, while this discrepancy is not observed for AST. obviously this could reflect a correspondingly large true variation in the proportion of released $C K$ and $H B D$. This is however cefuted by the moderate standard deviation of 248 found for the ratio of released $C K$ and released $H B D$, when cumulative release is calculated with fixed mean values for the circulatory parameters.

From the results obtained for simulated plasma activities, cf. Table 3.4, 3.10 and 3.11, it appears that the estimation of rho is especially sensitive for disturbances of the initial gtage, the first 12 hours, of the plasma activity curve. This offers a possible explanation for the huge variation in the rho ${ }_{\mathrm{CK}}$ : Even small perturbations of this initial phase, e.9. by non-cardiac CK release or a variable time delay of about one hour of release of $\mathrm{HBD}$ and $\mathrm{AST}$ compared to $\mathrm{CK}$ release, could cause comparable variation in the estimation of cho, while the total released quantity of CK is hardly affected. 
The values of the circulatory parameters found in this chapter are in agreement with the values published earlier by our group, based on different estimation procedures [Hermens, 1982; Willems, 1979; Willems, 19821. Therefore there is no necessity to change these earlier values, which are used in the sequel.

\section{Simplified_progedures}

In the previous chapter it was already demonstrated, cf. Table 3.4, that for the identification of the circulatory model parameters from simultaneous analysis of plasma activity curves it is required that (a) samples are taken at least up to ten days after the start of the infarction (b) one of the enzymes included in the analysis is catabolized slowly, compared to the extravasation rate, i.e. FCR $<0.05 \mathrm{~h}^{-1}$. These restrictions presumably arise from the gradual long lasting time course of enzyme release after AMI. While the first condition mainly represents an annoyance because of the large number of samples to be taken, the second condition represents an essential restriction as $H B D\left(L D_{1}\right.$ or $\left.L_{2} D_{2}\right)$ is the only cardiac enzyme that meets this requirement. Therefore it seems that the use of the simplified method to obtain estimations of FCR of rapidly eliminated enzymes by an analysis of the data of short time interval of two days and using HBD with fixed mean values for FCR, TER and ERR as a reference enzyme is preferable, once the circulatory model for HBD is identified. The resulting error in the estimation, due to neglection of the interindividual variation of the circulatory parameters of HBD is compensated by the better condition of the fit, cf. Table 3.4. 3.13 and 4.4 . In conclusion this simplified procedure is more convenient, exhibits no larger $\mathrm{CV}$ in the estimated parameters and does not lead to biased estimations.

Accuracy_of_guantification_of_cumulative release_of enzymes

A striking variation is present in all estimations of circulatory parameter values considered in this chapter. It is of obvious interest to determine which part of this variation is due to the application of the estimation procedure to data contami- 
nated with ercor and which part reflects true biological variation.

The data obtained in the better studies with radio-labelled plasma proteins indicate that coefficients of variation must be less than 20\%, see Section 2.4. The same magnitude of the coefficient of variation of FCR was found in experiments in the dog where enzymes were injected or infused in the plasma [Visser, 1981; Willems, 1982】. However such studies concern standarized groups of healthy subjects while the variation in patients with AMI, suffering from fever or hemodynamic disturbances may be larger.

Quantification of cumulative enzyme release after AMI, with fixed mean parameter values, cf. Table 4.8 , results howewer in a close correlation between the calculated release of the enzymes CK, HBD and AST. The coefficients of variation of 24 respectively $20 \%$ found for the ratio of released $C K$ and released $H B D$ and for the ratio of released AST and released HBD respectively do not allow a biological variation in FCR exceeding 15\%. This implies that the anticipated error in calculated error of cumulative release calculated with fixed mean values of the circulatory parameters is less than 15\%, cf. [Van der Laarse, 1984].

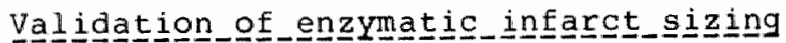

A direct validation of the use of enzymatic estimates of myocardial necrosis was first given by Erhardt, [Erhardt, 1974]. The author determined the peak levels of "thermostable LDA" sampled at 12 hourly intervals in patients with AMI. Heating of plasma samples as used in Erhardt"s study, inactivates the thermolabile isoenzymes $\mathrm{LDH}_{3}, \mathrm{LDH}_{4}$ and $\mathrm{LDH}_{5}$ and leaves $L D H_{1}$ and $\mathrm{LDH}_{2}$ intact. As shown in the next chapter this implies that measurement of thermostable $L D H$ is equivalent to the measurement of HBD, apart from a multiplicative factor. In 43 patients who died after the peak levels of $\mathrm{LDH}$ where reached, Exhardt determined the anatomical infarct size with the well known technique of staining heart slices with nitroblue tetrazolium (NBT). A good correlation between peak LDH activities and anatomical infarct size was found 
$(t=0.79)$. Since then similar validations have been reported for CK [Bleife1d, 1977] and CK-MB [Grande, 1982; Hacke1, 1984].

Although these studies have firmly established a correlation between myocardial necrosis and plasma enzyme activities after AMI, they do not settle controversy about the quantitative relation beween enyzme release and infacct size and especially the issue of the "plasma entrance ratio" "i.e. the fraction of enzyme depleted from the heart that is recovered in the plasma.

With the values of $F C R$ and TER and ERR obtained in this study the calculated release of the enzymes CK, HBD and AST is in proportion to the myocardial content of these enzymes (cf. Fig. 4.5 and Table. 4.8 ).

Moreover infarct sizes of $20(\mathrm{~g}$-eq/ 1$)$ are observed cf. Table 4.2. With a plasma volume of 31 and accounting for a residual enzyme activity of $20 \%$ in the central zone of the infarction this amounts to infarct sizes up to 75 gram. As such infarct sizes are observed in a group of patients with AMI uncomplicated by manifest cardiac failure it is concluded that infarct size is not underestimated to a significant extent and that local inactivation of enzymes is of limited importance in patients with AMI. From the data of Erhardt a similar conclusion can be drawn more directiy regarding LDH. It follows from our data that cumulative release of $L_{\Delta} H_{1}$ and $L_{L H}$ can be estimated by multiplication of the peak levels of these enzymes by a factor of 1.7 , cf. Fig. 4.1 and 4.3. Using this factor we calculated from Erhardt's data that per gram of infarcted myocardium, as measured by NBT staining, approximately $75 \mathrm{U}$ of $\mathrm{LDH}$ are released. Grande et al. [Grande. 1982] have shown that infarcted myocardium, as determined by NBT-staining has an overall enzyme depletion of approximately 50 \% with higher values (up to 85\%) in the centre and lower values towards the border of the infarcted zone. Using the value of 140 U/g for myocardial content of thermostable LDH (cf. Table 4.1) this implies that NBT-stained infarcted myocardium could maximalIy release about $70 \mathrm{~J} / \mathrm{g}$ of thermostable $\mathbb{L} D \mathrm{H}$ which is close to the value calculated from Erhardt's data. Apparently, local inactivation of thermostable LDH is quantitatively unimportant. Complete recovery in plasma of $\mathrm{CK}$ depleted from the heart is also indicat- 
ed by data presented by [Bleifeld, 1977]. In their study, an apparent disappearance rate constant $k_{d}=0.06 \mathrm{~h}^{-1}$ was used for $\mathrm{FCR}_{\mathrm{CK}}$ and it was assumed that $15 \%$ of the initial $\mathrm{CK}$ content of the infarcted myocardium was released in plasma. Using a value of $\mathrm{FCR}_{\mathrm{CK}}=0.20 \mathrm{~h}^{-1}$ the released fraction of $\mathrm{CK}$ is close to $50 \mathrm{~s}$ which is again in accordance with the overall depletion reported in the study of Grande. 


\section{CHAPTER 5}

\section{ENZYMATIC ASSESSMENT OF INJURY AFTER CARDIAC SURGERY}

\subsection{Introduction}

Soon after the first demonstration of increased plasma levels of cardiac enzymes in patients with acute myocardial infarction [LaDue, 1954], this phenomenon was also studied in patients after cardiac surgery [Nicke11, 1957]. Quantitative use of enzymatic data started when a correlation was reported between postoperative mortality and peak plasma levels of AST [Baer, 1960]. At that time, perioperative myocardial infarction was a common complication. Although it was realized that enzyme release after cardiac surgery is a complex response to anaesthesia, surgery and extracorporeal circulation [Baer, 1960, Hauss, 1958], it was concluded that a rapid rise of plasma activities of $A S T$ and $L D H$ during the first few postoperative hours, indicates evolving myocardial infarction [Welbourn, 1966; Dieter, 1969]. Since then, the introduction of improved anaesthetic and surgical techniques and the development of intraoperative myocardial protection, have drastically reduced the incidence of perioperative myocardial infarction [Kouchoukos, 1980]. Accordingly, myocardial enzyme release has been reduced considerably as we11 [Davids, 1982].

Enzymes of non-cardiac origin are not the only complication in enzymatic estimation of perioperative myocardial damage. The occurrence of hemodynamic changes in patients during the first few hours after termination of extracorporeal circulation may also necessitate corrections. During this period, there is a rapid reduction of plasma volume which has become considerably expanded during cardiopulmonary bypass [Welbourn, 1966]. The present study also demonstrates a largely increased transcapillary escape rate of protein in the first few postoperative hours.

In order to identify the various sources of extramyocardial enzyme release, a combination of (iso)enzymes is considered for which the activities present in myocardium, skeletal muscle and in blood cells were seperately determined. The release from 
Various tissues can thus be estimated from appropriate combinations of isoenzymes. It is shown that after uncomplicated cardiac surgery the release of creatine kinase (CK) and aspartate aminotransferase (AST) from skeletal muscle, as well as the release of lactate dehydrogenase (LDH) from erythrocytes, exceeds the myocardial release of these enzymes.

Perioperative hemodynamic changes are estimated in patients and, more accurately, in the dog. It is shown that these changes may also introduce large errors in the estimation of cumulative release of enzymes. The magnitude of these errors is largely deperident on the elimination rate of enzyme from plasma, on the extent of myocardial damage and, for alpha-hydroxybutyrate dehydrogenase (HBD), on the extent of peroperative hemolysis. 


\subsection{Methods}

Description_of_the_groups_of_patients_studied

Group A consists of 72 patients admitted in 1980 and 1981 to the Department of Cardiovascular surgery of the st. Raphael clinics in Leuven. These patients underwent aortocoronary bypass grafting in a randomised trial on three different techniques of myocardial protection. Details are published elsewhere [Flameng, 1984]. Perioperative enzyme levels were not different in these three groups and the data are pooled. After anaesthesia and sternotomy, a cardiopulmonary bypass (CPB) system was installed primed with $25 \mathrm{ml}$ per $\mathrm{kg}$ of body weight of a buffered solution containing 80-100 g of human serum albumin, 500 IU of heparin and $500 \mathrm{ml}$ of fresh blood. During $\mathrm{CPB}$ fresh blood was given in order to maintain hematocrit values above $25 \%$ (usually a total volume of $1-2$ 1). At the end of $C P B$, the oxygenator content was slowly transfused to the patients until a pulmonary artery wedge pressure of 8-9 $\mathrm{mm} \mathrm{Hg}$ was obtained. The blood balance was recorded after $C P B$ by measurement of the erythrocyte volume drained from the thoracic cavity (Argyle, Chest Drainage unit) and of the blood volume given in the postoperative period. Blood samples for the determination of $C K, C K-M B, H B D, A S T, H t$ and $H b$ were obtained at the start of $C P B$, immediately after $C P B$ and subsequently every 4 hours for 24 hours from an indwelling venous catheter. A final. blood sample was obtained 12 hours later. Group A represents recently performed, routine bypass surgery with limited myocardial damage.

Group B consists of 42 patients admitted to the Department of Thoracic Surgery, University Hospital, Leiden. Of these patients, 32 were admitted in 1975 and underwent aortocoronary bypass grafting, with intermittent aortic cross clamping at $32^{\circ} \mathrm{C}$, or prosthetic valve insertion, using coronary perfusion with cold blood. Blood samples for the determination of $\mathrm{CK}, \mathrm{CK}-\mathrm{MB}$, $\mathrm{HBD}$ and Ht were obtained as described for group A except for 6 hourly sampling during 48 hours postoperatively and thereafter 12 hourly sampling up to 96 hours. Further details are given elsewhere [Van 
der Laarse, $1979 a]$. An identical group of 10 patients was admitted in 1980 and protected peroperatively by potassium-induced hypothermic cardioplegia. Further details ace given elsewhere [Davids, 1982]. Blood samples were obtained every 4 hours until 48 hours after the start of CBB, then every 8 hours until 72 hours and every 12 hours unt1l 120 hours. These 10 patients were selected to include cases with considerable myocardial damage and an extensive (iso)enzyme analysis was performed, i.e. CK, CK-MB, $\mathrm{LDH}_{1}-\mathrm{LDH}_{5}$, cytoplasmic and mitochondrial AST, ALT and HBD were determined. Group B represents various forms of cardiac surgery with a high incidence of postoperative myocardial infarction, especially after aortic valve replacements [Van der Laarse, 1979 a].

Group C consists of 40 patients with acute myocardial infarction (AMI) admitted between 1975 and 1980 to the Coronary Care Unit of the University Hospital in Leiden, cf. patient group $A$ in Chapter 4.2 .

\section{An}

Mongrel dogs of both sexes and $15-25 \mathrm{~kg}$ of body weight were used. Five dogs were premedicated with fluanisone, $10 \mathrm{mg} / \mathrm{kg}$, and fentanyl citrate, $0.32 \mathrm{mg} / \mathrm{kg}$. Anaesthesia was induced with solium pentobarbital, $10 \mathrm{mg} / \mathrm{kg}$, and after endotracheal intubation maintained with nitrous oxide in oxygen. After sternotomy, a CPB system was 1 installed as used in patient group $A$. The composition of the priming solution and protocol for peroperative blood suppletion was also identical. After initiation of CBP, the rectal temperature was lowered to $25^{\circ} \mathrm{C}$ by means of the oxygenator heat exchanger. The hearts remained fibrillating throughout the hypothermic period. The aorta was cross-clamped during four periods of $10 \mathrm{~min}$ separated by $10 \mathrm{~min}$ intervals of reperfusion. After rewarming of the dogs and defibrillation when the rectal temperature reached $34^{\circ} \mathrm{C}, \mathrm{CPB}$ was terminated. The dogs were studied for another 120 minutes and killed with an overdose of sodium pentobarbital. 
Plasma volumes were determined by the enzyme dilution technique [Visser, 1982] before CPB and 10,70 and 120 min after CPB. For each determination, approximately 200 u of purified porcine AST (Sigma Chem. Co.) was injected into the left atrium.

In order to determine the transcapillary escape rate of protein in the postoperative period, an enzyme preparation, prepared by bypoxic incubation of dog liver [Visser, 1982] and containing approximately $200 \mathrm{U}$ of ALT per bolus, was injected immediately after CPB and the rate of disappearance of ALT from plasma was determined from 8 blood samples taken at $10 \mathrm{~min}$ intervals from a catheter in the brachial artery.

This liver enzyme preparation was also used to study the recovery in plasma of enzymes released in skeletal muscle. Intramuscular injections of $10 \mathrm{ml}$ were given in 9 dogs. In 4 cases, the liver preparation was mixed with a similar preparation obtained from dog heart [Visser, 1981cl with a high CK activity. Injections were given in the gluteus muscle and the AST, ALT and CK activities appearing in plasma were measured in serial blood samples taken from a catheter in the foreleg vein (vena cepha1is). Samples were obtained every 2 hours until 16 hours after the intramuscular injection, then every 4 hours until 40 hours and every 8 hours antil 72 hours.

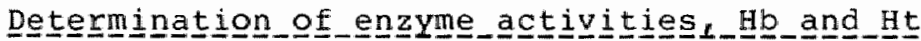

Blood samples consisted of $5-10 \mathrm{ml}$ of blood and clotting was prevented with sodium citrate. Samples were centrifugated immediately for $15 \mathrm{~min}$ at $1000 \mathrm{xg}$ in order to remove blood cells. In patient group $C$ centrifugation was repeated a second time for 20 min at $40,000 \mathrm{xg}$. Thereafter, plasma samples were stored at $-80^{\circ} \mathrm{C}$ (dogs and patient group $A$ ) or $-20^{\circ} \mathrm{C}$ (groups $B$ and $C$ ) until enzyme assays were perforned within 6 weeks after storage. Plasma enzyme activities of CK, CK-MB, LDH, LDH isoenzymes, AST, M-AST, HBD, ALT were assayed as described in Chapter 4.2. Hemoglobin (Hb) was determined spectrofotometrically at $540 \mathrm{~nm}$, using a test kit (Boerhringer) based on the cyanide method [Van Kampen, 1961 ] and expressed in mmol/1. Hematocrit (Ht) was determined immediately 
after collection of blood samples by the microcapillary method [McGovern, 1955]. Values are expressed as a percentage of total blood volume.

\section{Anaㄴysíg_of_data}

The time-activity curves of enzymes in plasma were analyzed with the two-compartment model shown in Fig. 5.1. The plasma pool $P(t)$ of enzyme at time $t$ is changed by metabolic degradation with a Eractional Catabolic Rate constant FCR, and by input of enzyme from the heart in plasma at a rate $F(t)$. In addition there is transport of enzyme from plasma to the extravascular pool of enzyme $E(t)$ with a fractional Transcapillary Escape Rate constant TER and return of enzyme from the extravascular pool to plasma with a fractional Extravascular Return Rate constant ERR. If it is assumed that the release of enzyme starts at time $t=0$, cumulative release of enzyme in plasma from $t=0$ up to time $t$ is given by:

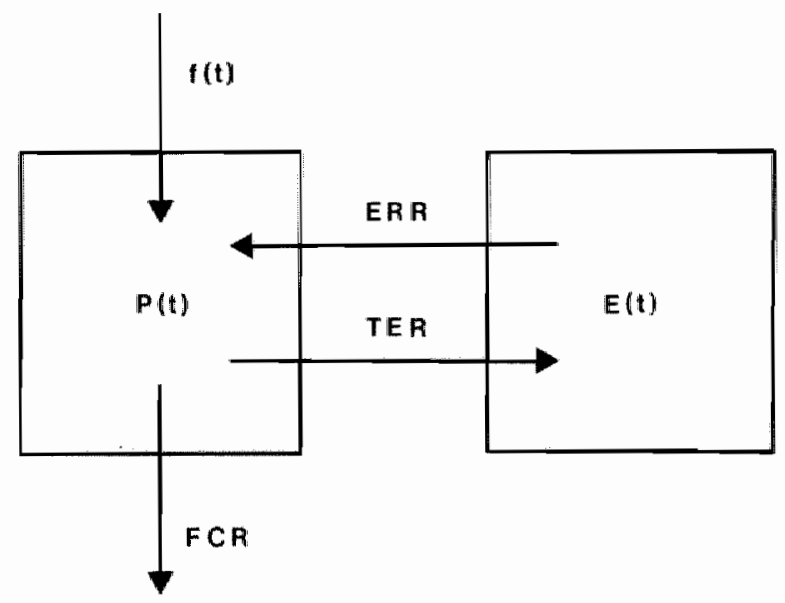

fig. 5.1 Two-compartment model for the calculation of cumulative release of ensymes from plasma ename activities (aee text). 


$$
A(t)=P(t)+E(t)+\int_{0}^{t} F C R P(\tau) d \tau
$$

where the first two terms give the quantities of enzyme which, after being released, are still present in plasma and in the extravascular space. The last term gives the quantity of enzyme catabolized up to time $t$. If the values of TER and ERR are known and $P(t)$ can be measuced as a function of time $t$, the term $E(t)$ in Equation 1 can be calculated from the equation [Willems, 1982 ; Hermens, 19821 :

$$
E(t)=\int_{0}^{t} \operatorname{TER}(\tau) \exp (-\operatorname{ERR}(t-\tau)) P(\tau) \mathrm{d} \tau
$$

The following parameter values, expressed in $\mathrm{h}^{-1}$, were used in Equations 5.1 and 5.2 [Willems, 1982; Visser, 1981c; Hermens, 1982 ; Chapter 41

in man: $\mathrm{FCR}_{\mathrm{CK}-\mathrm{MM}}=\mathrm{FCR}_{\mathrm{CK}}=0.20 ; \mathrm{FCR}_{\mathrm{CK}-\mathrm{MB}}=0.34 ; \mathrm{FCR}_{\mathrm{HBD}}=0.015 ;$ $\mathrm{FCR}_{\text {CAST }}=\mathrm{FCR}_{\mathrm{AST}}=0.093 ; \mathrm{FCR}_{\mathrm{ALT}}=0.042$;

$\mathrm{TER}=0.014 ; \mathrm{ERR}=0.018$.

in dogs : $\mathrm{ECR}_{\mathrm{CK}}=0.36 ; \mathrm{FCR}_{\mathrm{AST}}=0.21 ; \mathrm{FCR}_{\mathrm{AL} \mathrm{T}}=0.022$; TER $=0.031 ; \mathrm{ERR}=0.064$.

Values of $P(t)$ were determined by use of the relation $P(t)=$ $V(t) . C(t)$ where $V(t)$ is the plasma volume at time $t$ and $C(t)$ is the plasma enzyme activity at time $t$ from which the normal steady state plasma enzyme acitivity CS is subtracted. For the rapidy catabolized enzymes $C K$ and AST, individual values of CS were used, as measured before $\mathrm{CPB}$ in the surgery groups and after renormalization of plasma activities in the infarction group. For the slowly catabolized enzymes HBD and ALT, fixed mean values of respectively $\mathrm{CS}=80 \mathrm{U} / 1$ and $\mathrm{CS}=7 \mathrm{U} / 1$ were used.

Postoperative plasma volume changes can be estimated from changes in hematocrits. In individual patients this procedure is subject to error as the loss of erythrocytes due to bleeding may 
not be exactly compensated by blood transfusion. However, from the blood balance records averaged for the whole group of patients it is concluded (cf. Results) that such compensation did occur. Therefore, mean values of $V(t)$ were calculated from the mean hematocrit values according to the equation fHermens, 1982; Van Beamont, 1974]:

$$
\bar{V}(t)=\bar{V}(0)[100 / \bar{H} t(t)-1] /[100 / \bar{H} t(0)-1]
$$

where $\bar{V}(t)$ and $\bar{H} t(t)$ are respectively the mean plasma volume and the mean hematocrit value at time $t$. Equation 5.3 follows from the relation $V(t) / V E=(100-H t(t)) / H t(t)=100 / H t(t)-1$, where $\mathrm{VE}$ is the total erythrocyte volume which is assumed to remain constant between $t=0$ and $t$ ime $t$. The mean normal values $\bar{V}(0)$ and $\overrightarrow{\mathrm{H}} \mathrm{t}(0)$ were estimated from body weight, sex and age [Geigy, 1979] at respectively 2.981 and 44.28 . 



\subsection{Results}

\section{Signifífance_of_plasma_enzyme_activitity_measurements}

Table 5.1 shows the enzyme content of various tissues that may be damaged during cardiac surgery. It is apparent that multienzyme or isoenzyme analysis could be useful in the identification of various sources of enzyme release. Total enzyme activities can be measured more simply and accurately than isoenzyme activities because the separation of isoenzymes is often laborious and may introduce considerable error. However, application of Equations 5.1 and 5.2 to total plasma enzyme activities may also lead to error because total plasma activity of e.g. CK is composed of $\mathrm{CK}-\mathrm{MB}$ and CK-MM activity, i.e. different molecular forms with different values of FCR. Therefore, it must be first ascertained under which conditions the application of Equations 5.1 and 5.2 to total plasma activities is meaningfull.

\section{Meas}

The LDH molecule is a tetramer composed of two different subunits: $H$ (heart) and M(muscle). The following combinations exist: $\mathrm{H}_{4}\left(\mathrm{LDH}_{1}\right), \mathrm{H}_{3} \mathrm{M}\left(\mathrm{LDH}_{2}\right), \mathrm{H}_{2} \mathrm{M}_{2}\left(\mathrm{LDH}_{3}\right), \mathrm{HM}_{3}\left(\mathrm{LDH}_{4}\right)$ and $\mathrm{M}_{4}\left(\mathrm{LDH}_{5}\right), \mathrm{Fig} .5 .2$ shows mean activities of total $L D H$ and of the LDH isoenzymes measured after AMI in patient group $C(n=10)$ and after cardiac surgery in patient group $B(n=10)$. In the surgery group corrections for peroperative hemolysis and perioperative hemodynamic changes as described below were applied. After AMI, release of $\mathrm{LDH}$ consist almost exclusively of $\mathrm{LDH}_{1}$ and $\mathrm{LDH}_{2}$, as should be expected from the data in Table 5.1 showing that less than 108 of total myocardial LDH content consists of the isoenzymes $\mathrm{LDH}_{3}$, $\mathrm{LDH}_{4}$ and $\mathrm{LDH}_{5}$. However, it appears that the release of $\mathrm{LDH}_{4}$ and $\mathrm{LDH}_{5}$ is also minimal after cardiac surgery in spite of damaged skeletal muscles (cf. below) with a high $\mathrm{LDH}_{5}$ content (cf. Table 5.1). As discussed below, this indicates either local inactivation or rapid elimination from plasma of $\mathrm{LDH}_{5}$.

Cumulative release of LDH obtained from Equations 5.1 and 5.2 with $F C R=0.015 \mathrm{~h}^{-1}$, is closely approximated by the summed acti- 
Table 5.1 Enzyme content of human muscle and blood.

\begin{tabular}{|c|c|c|c|c|c|c|c|}
\hline En- & Heart ${ }^{1}$ & Skel & tal muscl & $e^{2}$ & Blor & $s d^{3}$ & \\
\hline zyme & & aiaphr. & interc. & pect. & erythr. & platel. & leuc. \\
\hline $\mathrm{CK}$ & $865 \pm 10$ & $2377 \pm 14$ & $3.110+10$ & $2580 \pm 38$ & $1.7 \pm 12$ & $0.01+33$ & $0.02+34$ \\
\hline$C K-M B$ & $132+27$ & $36+200$ & $28+100$ & $15+200$ & not & detectabl & \\
\hline $\mathrm{HBD}$ & $123 \pm 9$ & $48 \pm 25$ & $69 \pm 12$ & $42+43$ & $11.7 \pm 9$ & $0.26+15$ & $0.26+25$ \\
\hline LDER & $155+8$ & $113 \pm 18$ & $266 \pm 13$ & $138 \pm 33$ & $16.5 \pm 8$ & $0.60+27$ & $0.65+24$ \\
\hline $\mathrm{LDH}_{1}$ & $84 \pm 10$ & $15 \pm 20$ & 0 & $1 \pm 900$ & $7.1 \pm 5$ & $0.06+70$ & $0.06+25$ \\
\hline $\mathrm{LDH}_{2}$ & $57+9$ & $14 \pm 21$ & $6 \pm 22$ & $5 \pm 64$ & $6.6+18$ & $0.19+10$ & $0.14 \pm 10$ \\
\hline $\mathrm{LDH}_{3}$ & $12 \pm 17$ & $14+71$ & $19+42$ & $5 \pm 100$ & $2 \cdot 6+44$ & $0.22 \pm 6$ & $0.15+20$ \\
\hline $\mathrm{LDH}_{4}$ & $3+33$ & $21 \pm 43$ & $43+19$ & $13 \pm 23$ & $1.7+99$ & $0.13 \pm 33$ & $0.15 \pm 11$ \\
\hline $\mathbf{L D H}_{5}$ & 0 & $48 \pm 31$ & $200 \pm 7$ & $113 \pm 6$ & 0 & $0.01 \pm 50$ & $0.15+12$ \\
\hline AST & $146 \pm 11$ & $62+11$ & $55 \pm 6$ & $48 \pm 33$ & $0.3 \pm 12$ & $0.01 \pm 14$ & $0.02+30$ \\
\hline mAsT & $92 \pm 12$ & $29 \pm 28$ & $22+23$ & $19 \pm 26$ & not & detectabl & \\
\hline LT & $5 \pm 37$ & $6 \pm 48$ & $3 \pm 22$ & $6 \pm 62$ & not & determine & \\
\hline
\end{tabular}

Figures indicate mean activities \pm coefficient of variation( 8 ) per gram of muscle or per ml of blood. Activities were determined at $25^{\circ} \mathrm{C}$ after homogenization and sonification of samples in buffered saline. Details are described in [Van der Laarse, 1980b; Hollaax, 1979].

(1) Determined in biopsies $(n=8)$ obtained during surgery. No significant differences were found between left and right ventxicular enzyme content [Van der Laarse, 1980b].

(2) Determined in diaphragm $(n=6)$, pectoral $(n=4)$ and intercostal $(n=3)$ muscle biopsies obtained during surgery.

(3) Calculated from [Hollaar, 1979] for cell counts of $5 \times 10^{9}$ erythrocytes per ml, $4 \times 10^{8}$ platelets per ml and $7 \times 10^{6}$ leucocytes (628 granulocytes and $38 \%$ lymphocyteg * monocytes) per ml. 
U/1

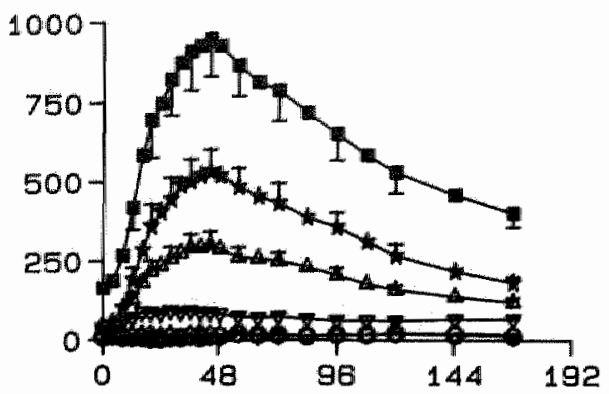

u

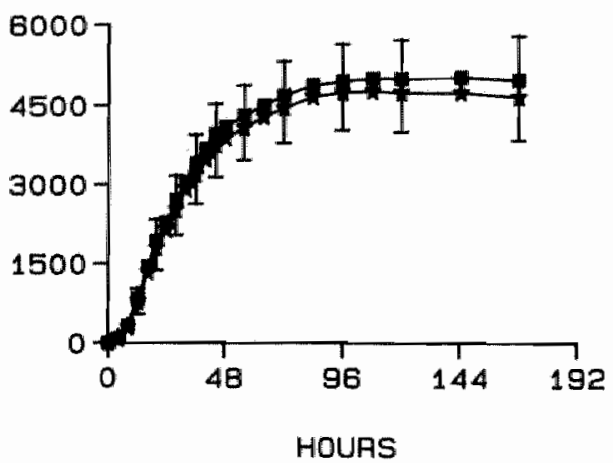

$U / 1$

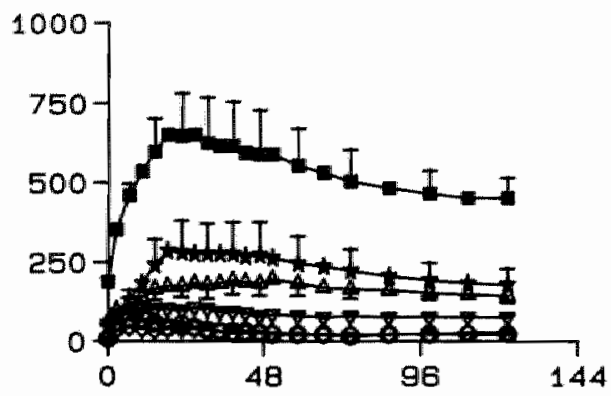

u

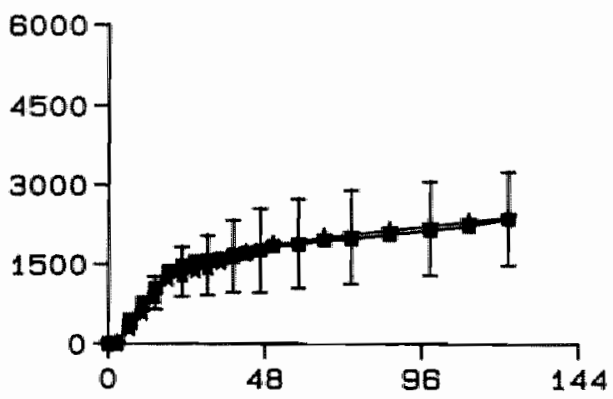

HOURS

Fig. 5.2 Contributions of $L D H_{1}(\star), L D H_{2}(\Delta), L D H_{3}(\nabla), L D H_{4}(\diamond)$ and $L D H_{5}$ (O) to total plasma LDH activty ( $(\mathbf{)}$. Left panet: after AMI. Right panel: after cardiac aurgery. Bottom figures show the summed contribution of $L D H_{1}$ and $L D H_{2}$ ( $\$ to cumulative release of total $L D H$ activity (1). Mean values \pm SEM are shown in all Figures.

vity of $\mathrm{LDH}_{1}$ and $\mathrm{LDH}_{2}$. The strictly parallel time course of the $\mathrm{LDH}_{1}$ and $\mathrm{LDH}_{2}$ plasma activity curves (cf. Fig. 5.2) confirms that both isoenzymes must have approximately equal values of FCR, cf. Table 4.6 . 
$U / 1$

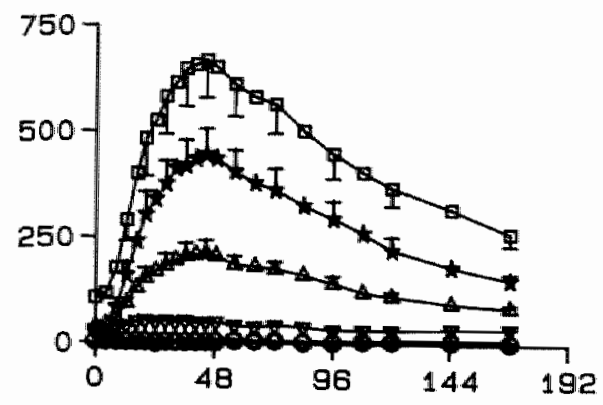

u

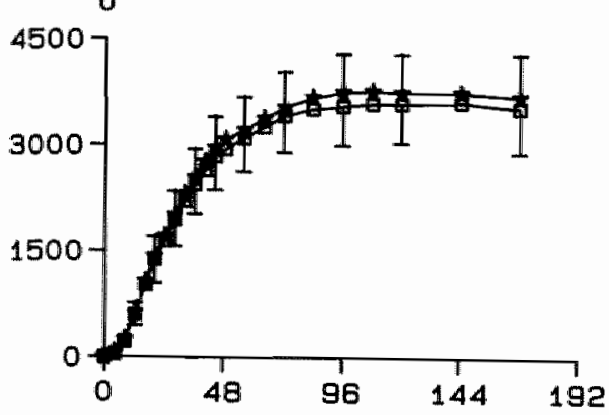

HOURS
$0 / 1$

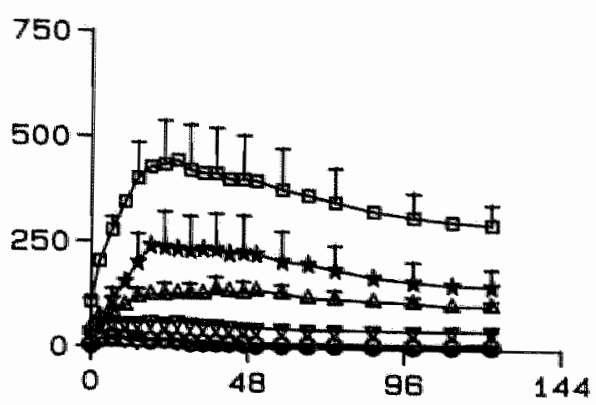

U

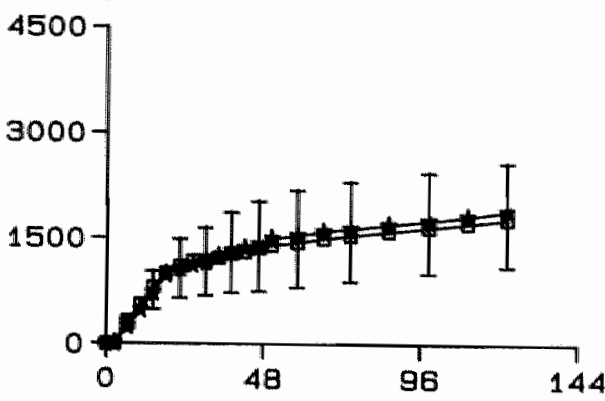

HOUAS

Pig. 5.3 Contributions of $L D H_{1}(\star), L D H_{2}(\Delta), L D H_{3}(\nabla), L D H_{4}(\Theta)$ and $L D H_{5}$ (O) to total polarma $H B D$ activity ( $(0)$. Left panel: after AMI. Right panel: after cardiac augery. Bottom figurea show the surmed contribution of $L_{D H}$ and $L_{2} H_{2}(\star)$ to cumulative rezease of totat $H B D$ activity (a).

$\left(\mathrm{CH}_{3} \mathrm{CH}_{2} \mathrm{COCOOH}\right)$, which is the substrate in the HBD assay, is based on the close resemblance to pyruvate ( $\mathrm{CH}_{3} \mathrm{COCOOH}$, the natural. substrate of LDH. However, HBD activity is particularly a property of the H-subunits of the LDH molecule. The HBD activities of the various LDH isoenzymes, expressed as a percentage of their activity in the LDH assay, are $84 \%, 71 \%, 57,43 \%$ and $30 \%$ for $\mathrm{LDH}_{1}, \mathrm{LDH}_{2}, \mathrm{LDH}_{3}, \mathrm{LDH}_{4}$ and $\mathrm{LDH}_{5}$ respectively lVan der Laarse, 1980b; Hollaar, 1979]. 
As a result the plasma activities in Fig. 5.3 are even more dominated by $\mathrm{LDH}_{1}$ and $\mathrm{LDH}_{2}$ than in Fig. 5.2. The cumulative release of total HBD again is closely approximated by the contributions of $\mathrm{LDH}_{1}$ and $\mathrm{LDH}_{2}$ *

\section{Measurement_of AsT}

In the myocardial cell, AST is located in the cytoplasm (CAST) and in the mitochondria (mAST) (CE. Table 5.1). After rupture of the cell membrane CAST is readily released and the bulk of plasma AST activity after AMI consists of CAST. Part of the mAST pool is

$\mathrm{U} / 1$

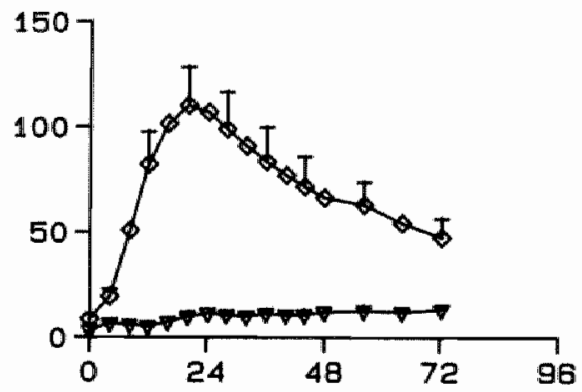

U

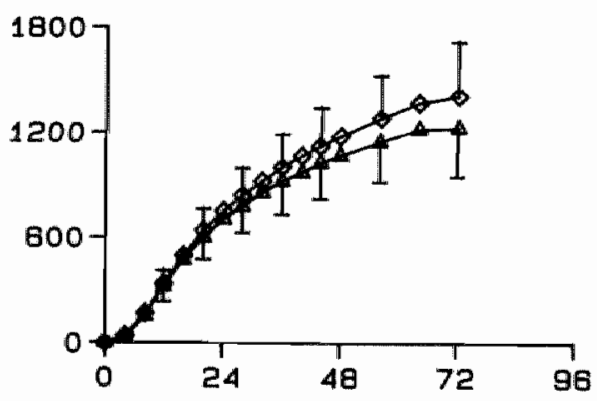

HOUIRS
$\mathrm{U} / 1$

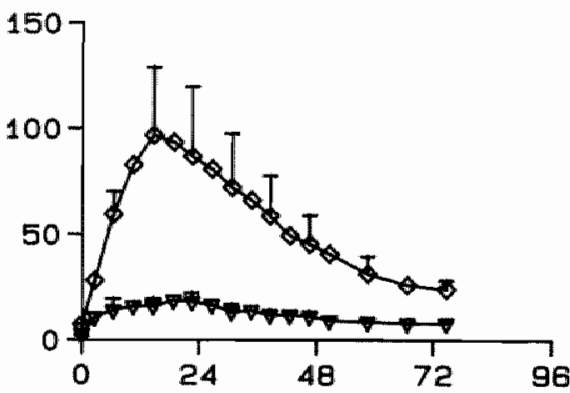

U

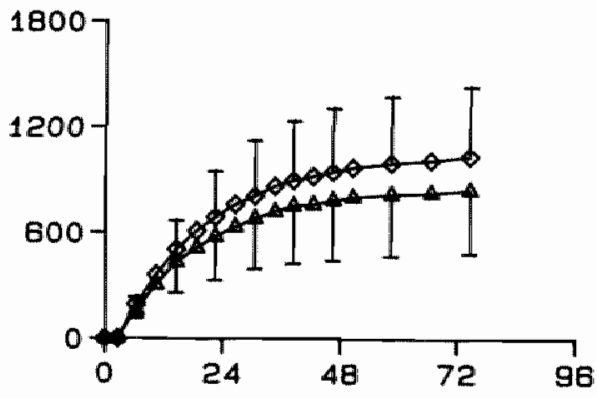

HOUAS

Fig. 5.4 Contribution of MAST ( $\nabla)$ to total plama AST activity (O). Left panel: after AMI. Right panel: after cardiac surgery. Bottom figures show the contribution of $C A S T(\Delta)$ to cumulative release of total $A S T$ activity $(0)$. 
firmly attached to mitochondrial structures and is not released by the dying cardiac cell [Van der Laarse, 1981]. The remaining part of the mAST pool is located in the intermembrane space of the mitochondrion and may be released after AMI. As shown in Fig. 5.4 for patients with AMI, this release of mAST is delayed and incomplete in comparison with release of cAST. It follows from Fig. 5.4 that cumulative release of AST, calculated from total plasma AST activities and a value of ECR $=0.093 \mathrm{~h}^{-1}$, can be used as an approximation of cumulative release of cAst with an overestimation of about $10 \%$ after AMI and about $16 \%$ after cardiac surgery.

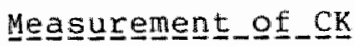

The CK molecule is a dimer, composed of two subunits: M (muscle) and $B$ (brain). The predominant form in heart and in skeletal muscle is CK-MM. Approximately 15 of total CK activity in the heart consists of $C K-M B$, while this percentage is less than 18 in skeletal muscle, except for diaphragm (cf. Table 5.1). The quantity of $C K-B B$ in heart and skeletal muscle is negligeable. Apart from the cytosolic isoenzymes, muscle alsa contains a small fraction of mitochondrial $\mathrm{CK}$ which is however quantitative1y unimportant in the calculation of cumulative release of CR in plasma [Grace, 1983]. Fig. 5.5 shows total CK and CK-MB activities in plasma of patients after $A M I$ and after cardiac surgery. It is also shown in Fig. 5.5 that the release of CK, calculated from total plasma $C K$ activities and a value of $F C R=0.20 \mathrm{~h}^{-1}$, closely appoximates the celease of CK-MM, i.e. the overestimation is of the order of $8 \%$ after AMI and even less after cardiac surgery. A continuing release of CK in the surgery group, due to skeletal muscle damage (cf. below) is apparent in Fig. 5.5 .

Perioperative changes in circulatory parameters

Transiently increased plasma_volume

Fig. 5.6 shows serial determinations of plasma volume by means of bolus injections of AST in a dog. From the large volumes of 
$U / 1$

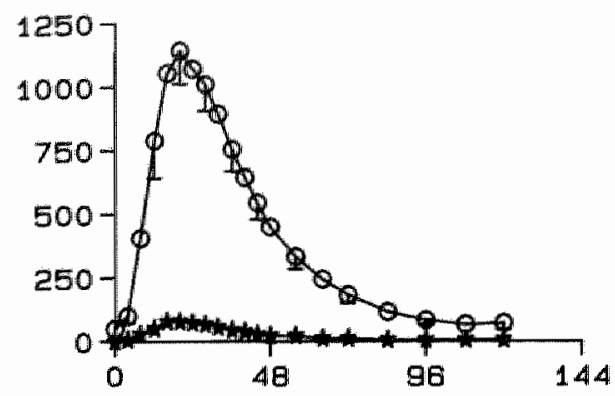

u

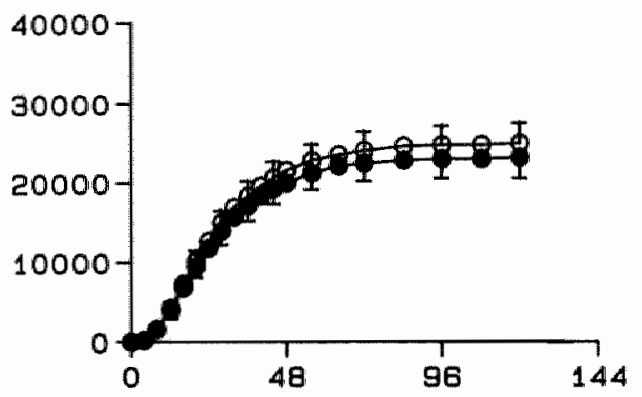

HOURS
$U / 1$

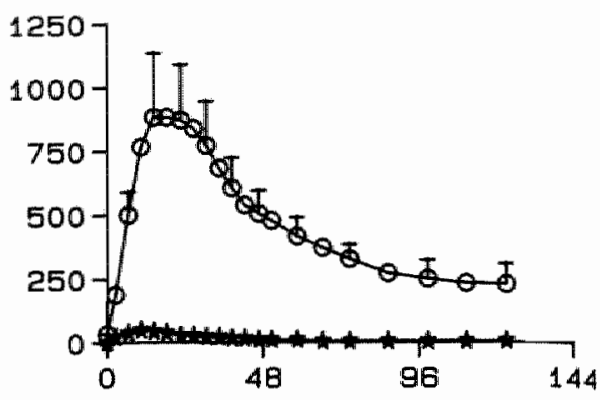

u

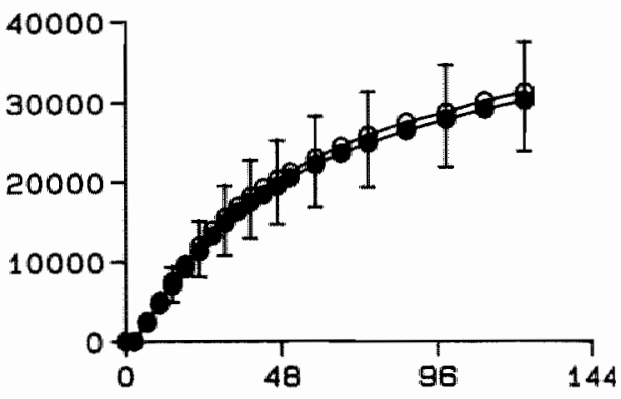

HOURS

Pig. 5.5 Contribution of $C K-M B$ ( $\star$ ) to total plama $C K$ activity $(O)$. Left panel: after AMI. Right panel: after cardiac burgery. Bottom figures show the contribution of $C K-M M$ ( $)$ to cumutative release of total $\mathrm{CK}$ activity $(\mathrm{O})$.

diluted oxygenator blood transfused to patients and dogs after CPB, it follows that a considerable expansion of plasma volume must have occurred, as shown in Fig. 5.7. This Figure also shows the mean post-CPB plasma volume changes in patients from group $A$, as calculated from the mean haematocrit values and Equation 5.3. As explained in the Section 5.2, this procedure assumes that the average erythrocyte volume remains constant after CPB. Indeed, the blood balance records demonstrated that, although these patients received $1.1 \pm 0.5$ (mean \pm SD) litre of blood during the first 24 hours after CPB, the averaged net blood balance was only 


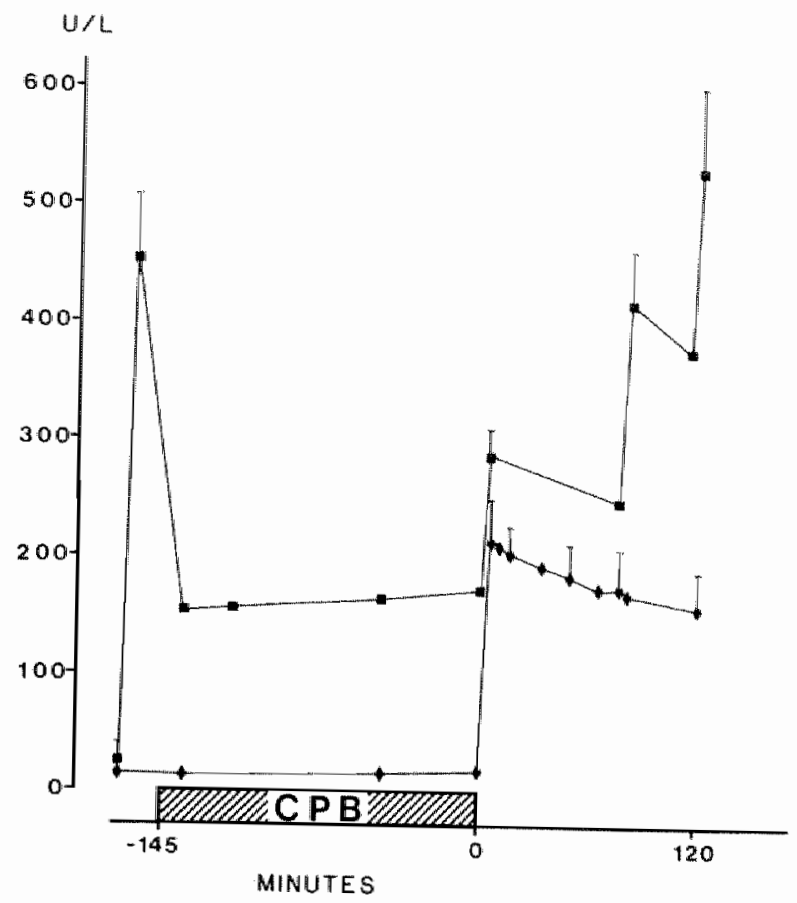

Fig. 5.6 Serial determination of ptasma votume, from botus injections of AST $(\mathbf{\omega})$, after CPB in the dog. A single loading dose of $A L T$ ( $\downarrow$ ) is given just after $C P B$ for the measurement of transcapilzary protein escape.

+ $85 \mathrm{ml}$ of blood. Fig. 5.7 shows that the largely expanded plasma volume after CPB rapidly renormalizes in the first few post-operative hours.

\section{Transient1y_increased_transcapillary_escape_of_protein}

Fig. 5.8 shows the accellerated disappearance of enzymes from plasma during the phase of rapidly decreasing plasma volume. The plasma pool $P(t)$ was calculated from the plasma activities $C(t)$ and the mean plasma volumes $\bar{v}(t)$ as shown in Fig. 5.7. The normal disappearance constant of ALT during the first few hours after 


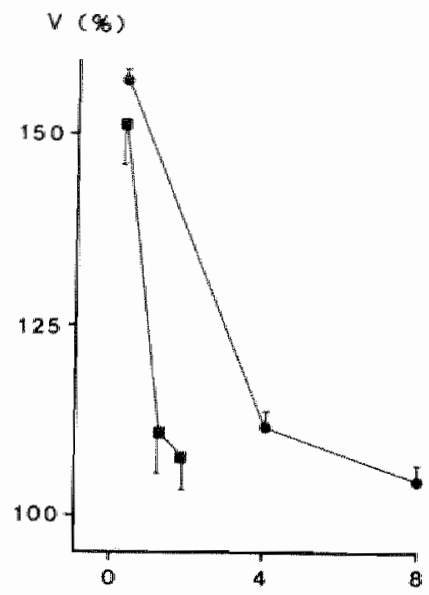

HOURS

Fig. 5.7 Plasma votume ehanges after CPB measured in patient group A (O) and in the dog (a). Values ame expressed as a percentage of nomat. values.

bolus injection in the $\operatorname{dog}$ is $k_{\mathrm{d}}=0.053 \mathrm{~h}^{-1}$ [Willems, 1982] while a value of $\mathrm{k}_{\mathrm{d}}=0.48 \pm 0.08 \mathrm{~h}^{-1}$ (mean $\pm S E, \mathrm{n}=5$ ) was found For the bolus of ALT injected after CPB (cf. Fig. 5.6). Similarly, the mean disappearance rate of HBD observed in patients after AMI cannot exceed the theoretically maximal value $\mathrm{k}_{\mathrm{d}}=\mathrm{FCR}_{\mathrm{HBD}}+$ TER $=0.029 \mathrm{~h}^{-1}$ in normal steady state conditions and a value of $\mathrm{k}_{\mathrm{d}}=0.011 \mathrm{~h}^{-1}$ is actually observed after AMI (cf. Fig. 5.8). In contrast, a mean value of $k_{\mathrm{d}}=0.089 \mathrm{~h}^{-1}$ was found in the first hours after CPB in patients of group A (CE. Fig. 5.8). Correction for the catabolic elimination of HBD from plasma would result in a value of $\mathrm{RER}=\mathrm{k}_{\mathrm{d}}-\mathrm{FCR}_{\mathrm{HBD}}=0.089-0.015=0.074 \mathrm{~h}^{-1}$. However, there is postoperative release of myocardial HBD and the actual walue of TER must therefore be higher. This release was estimated as described below, using a normall value of $\mathrm{TER}=0.014$ $h^{-1}$ as a first approximation, and values of TER were corrected accordingly. The final values for TER thus obtained are TER = $0.100 \mathrm{~h}^{-1}$ for the first 4 hours after CPB and TER $=0.057 \mathrm{~h}^{-1}$ for the next 4 hours. These values for TER, and plasma volumes as shown in Fig. 5.7, were used in the calculations of $A(t)$ for patients after cardiac surgery. 

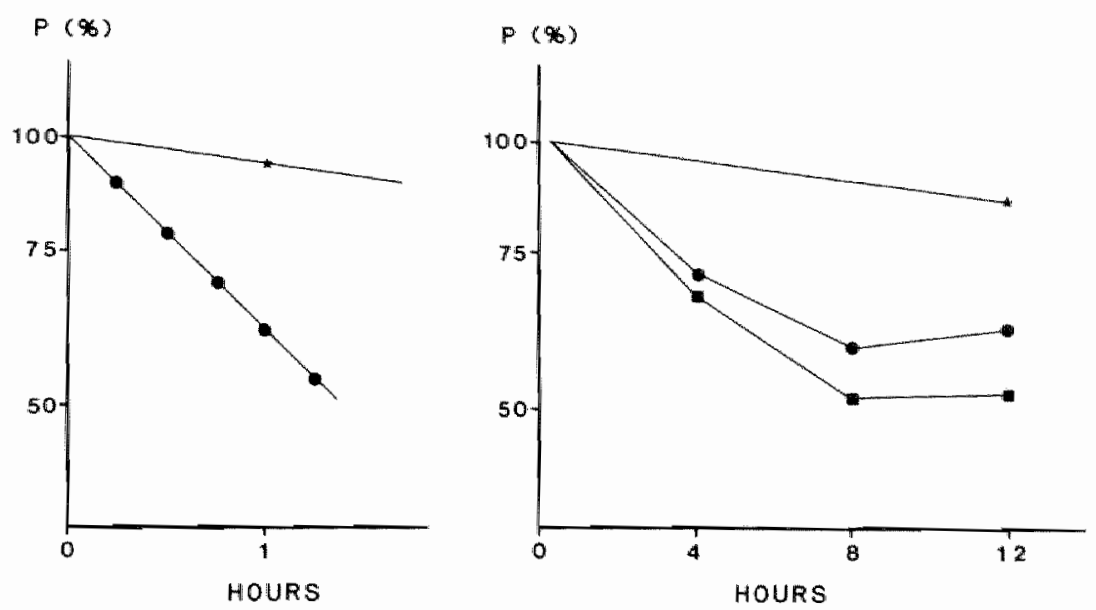

Fig. 5.8 POBt-CPB disappearance of ALT(-) in the dog (left figure) and of $H B D(\bullet)$ in patient group A (might figure). Plasma pools $P(t)$ are expressed as a percentage of the values found direatly after CPB (Zogarithmic scale). Normal disappearance rates of ALT ( $\star$ ) after botus injection in the dog, and of $B B D(*)$ after $A M T$ in patiente, are also shown. The right figure shows the disappearance of $H B D$ after correction for catabolism and myocardial release ( $\mathbf{n}$, see text).

Contribution of various tissues to perioperative_enzyme_redease Éstimation ó bemolysis

Fig. 5.9 shows that during CPB a variable but sometimes considerable increase in free plasma Hb concentrations and plasma HBD activities is observed. The slope of the best-fit straight line in Fig. 5.9 is $C_{H B D} / H b=1.10$ U/micromole, while the catio of $\mathrm{HBD}$ activity to $\mathrm{Hb}$ concentration in a preparation obtained by freezing and sonification of the packed cell sediment in centrifugated blood samples, is $C_{H B D} / H b=1.28 \pm 0.13 \mathrm{~J} / \mathrm{micromole}$ (mean $\pm S D)$. It follows from Table 5.1 that this value is only marginal1y influenced by the presence of leucocytes and platelets in the packed cell preparation. Hemolysis during CPB apparently 


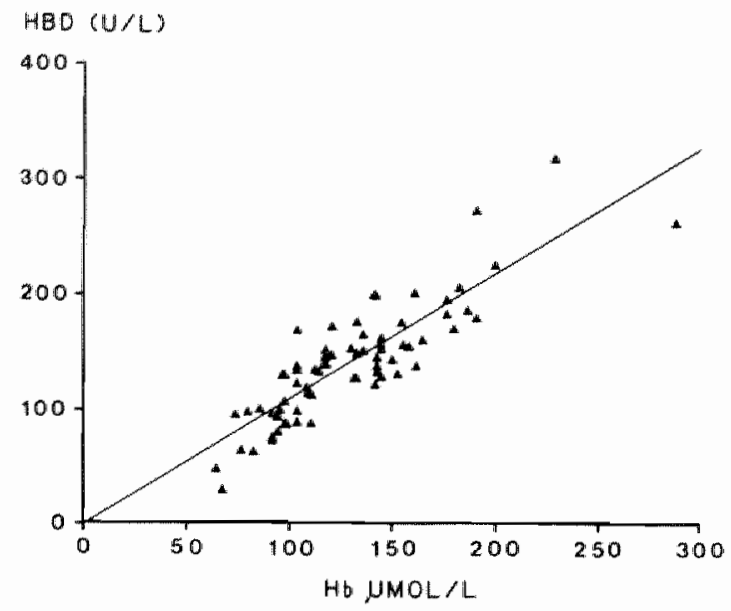

Fig. 5.9 Comelation between the peroperative inerease in plasma HBD activity and the eimilar increase in the free plasma hemoglobin concentration. Data are from patient group $A$. The correlation coefficient $i_{6} 0.87$.

leads to complete liberation of HBD from the damaged erythrocytes. From the mean increase of $795 \pm 175 \mathrm{u}$ of HBD observed during $\mathrm{CPB}$, it is concluded (cf. Table 5.1) that $68 \pm 15 \mathrm{ml}$ of blood is hemolyzed. As discussed below, there is no indication for continuing hemolysis after CPB. Corrections for hemolysis were therefore made by subtracting the peroperative increase in the circulating quantity of HBD from calculated values of $A(t)$.

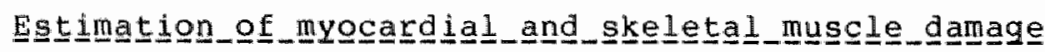

Fig. 5.10 shows cumulative release of enzymes in patient groups $A, B$ and $C$, expressed in gram-equivalents of myocardium, i.e. calculated values of $A(t)$ were divided by the enzyme content per gram of myocardium (cf. Table 5.1). After AMI, approximately equal estimates of myocardial damage are obtained from $\mathrm{HBD}, \mathrm{CK}$, and AST while the release of $C K-M B$ falls somewhat behind in the course of time. It seems as if in these patients $\mathrm{CK}-\mathrm{MB}$ is init- 

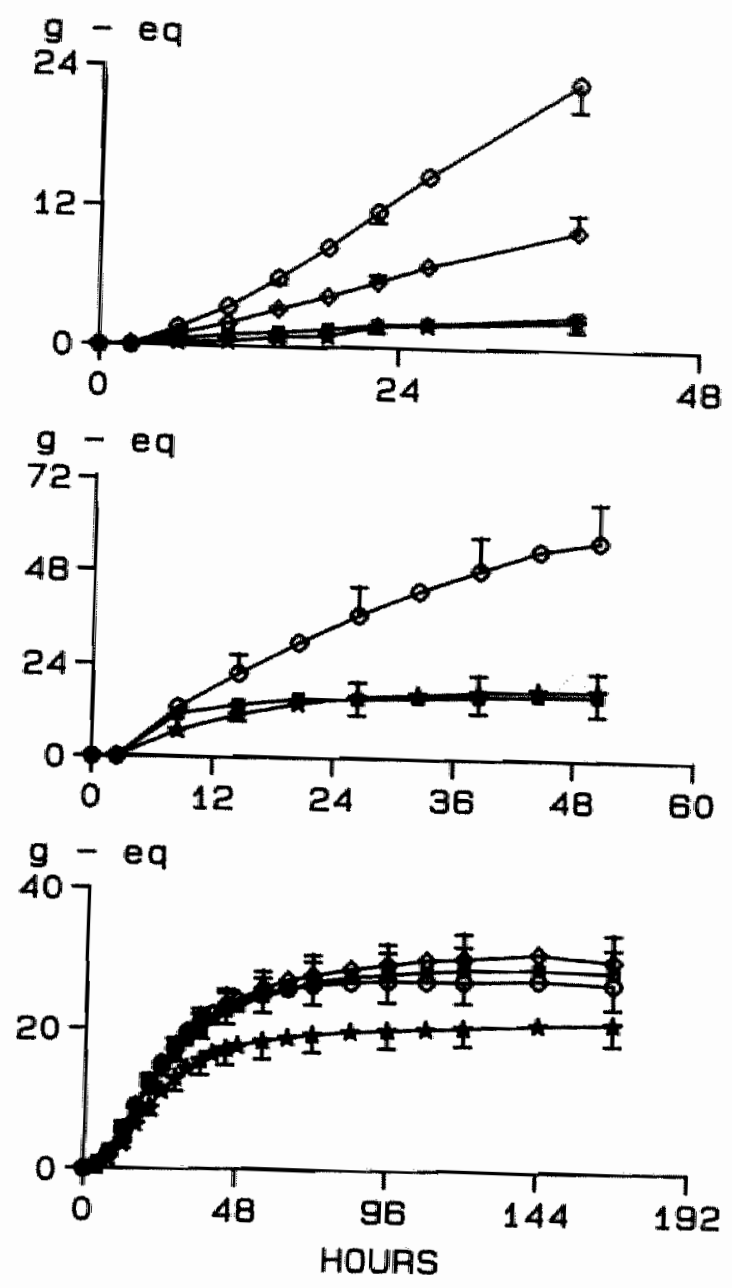

Fig. 5.10 Cumlative release of $H B D(\square), C K(0), C K-M B$ ( $(\star)$ and AST (O) after uneventfulz bypass surgery (group $A$; upper figure), after cardiac surgemy with frequent postoperative infaratione (group $B$; middie figure) and after AMI (Bottom figurel. Coracetione for peroperative hemolysis were performed for HBD. Extra release of $C K$ and AST from sketetal musele is apparent in the surgery groups 

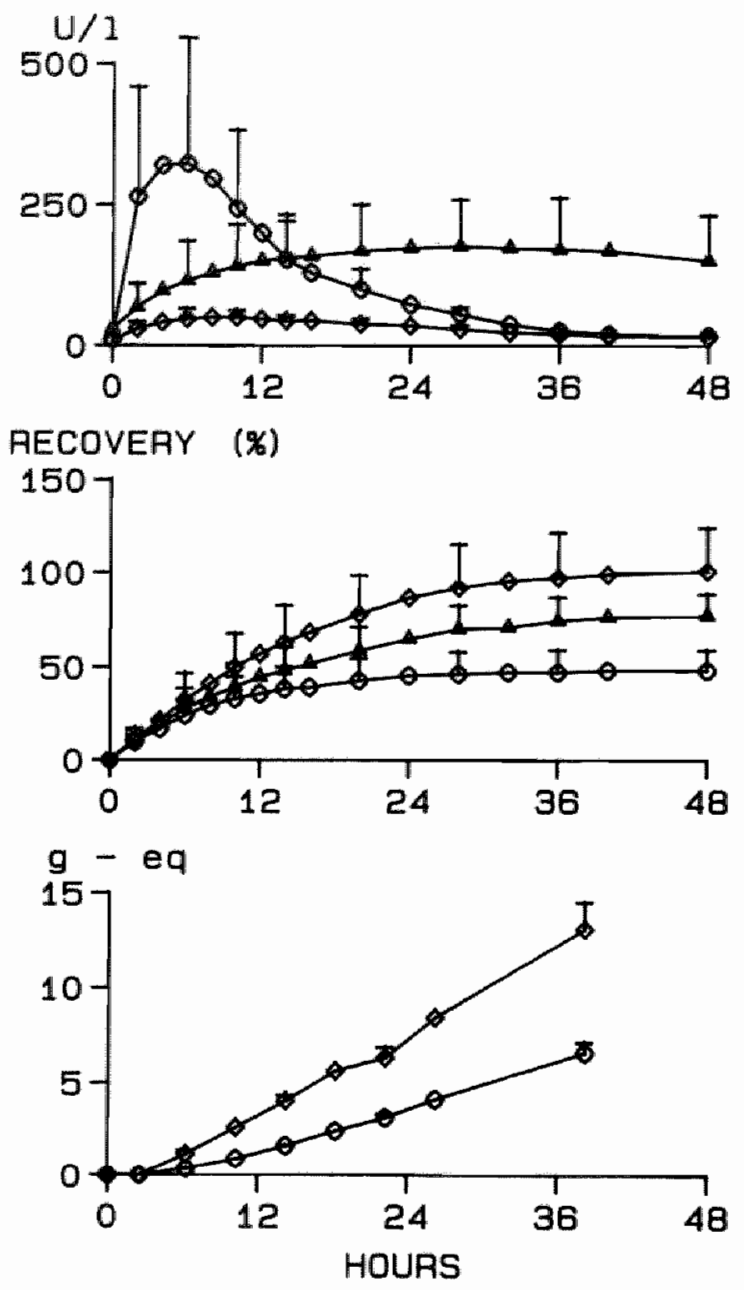

Fig. 5.11 Plasma activities (upper figure) and recovery in plasma (middle figure) of $C K(O)$, $A S T(D)$ and $A L T(\Delta)$ after intramuscultar injection of these ensymes in the dog. The recovery is the cumlative release in plasma expressed as a percentage of the injected quantities of enzyme. The bottom figure presents the lass of skeletal muscle as estimated from $C K$ and from $A S T$ in group $A$. 
ially released in proportion to the other enzymes but that this release gradually lags behind, leading to a significant difference after 48 hours. Total myocardial damage in group $C$ was estimated from HBD at $31 \pm 13$ (mean \pm SD) grams of myocardium.

Fig. 5.10 shows that after cardiac surgery HBD foorrected for peroperative hemolysis) and $\mathrm{CK}-\mathrm{MB}$ are released in proportion to myocardial enzyme content. This release is maindy restricted to the first 36 hours after surgery. As shown before, skeletal muscle damage does hardly contribute to release of HBD and mean myocardial damage may therefore be estimated from $H B D$ and $C K-M B$ at $1.5 \pm 1.5 \mathrm{~g}$-eq in group $A$ and $16 \pm 24 \mathrm{~g}$-eq (mean $\pm \mathrm{SD}$ ) in group $B$. The high value in patient group $B$ was mainly caused by the considerable damage observed after aortic valve replacements with the non-optimal cardiac preservation techniques used at that time [Davids, 1982]. Postoperative hemolysis would result in increased release of HBD compared to $C K-M B$, which is not observed.

Skeletal muscle damage after surgery is estimated by subtracting the myocardial contribution to $\mathrm{CK}$ and $\mathrm{AST}$ release lestimated by means of HBD release) from the abserved total release of these enzymes after surgery,cf. Fig. 5.10. The so calculated muscle damage expressed in gram equivalents of skeletal muscle by dividing by the mean values for skeletal muscle content of CK and AST from Table 5.1 is shown in Fig. 5.11. Appearantly there exists a discrepancy between the size of the skeletal muscle injury estimated Erom $C K(6.5 \pm 4.2 \mathrm{~g}-\mathrm{eq})$ and the size of this injury estimated from AST $(13 \pm 109-e q)$. This discrepancy in the extent of skeletal muscle damage, as estimated from CK or AST, was further investigated in the dog. Fig. 5.11 shows that AST, injected intramuscularly in the dog, is recovered completely in plasma while $\mathrm{CK}$ is only cecovered for approximately 50\%. Apparently, a large fraction of the injected $C K$ is inactivated during transport from the muscle to plasma. Although extrapolation to man has to be considered with great caution, this result offers a tentative explanation for the just mentioned discrepancy and indicates that skeletal muscle damage can more safely be estimated from AST than from CK. 
$9-e q / n$

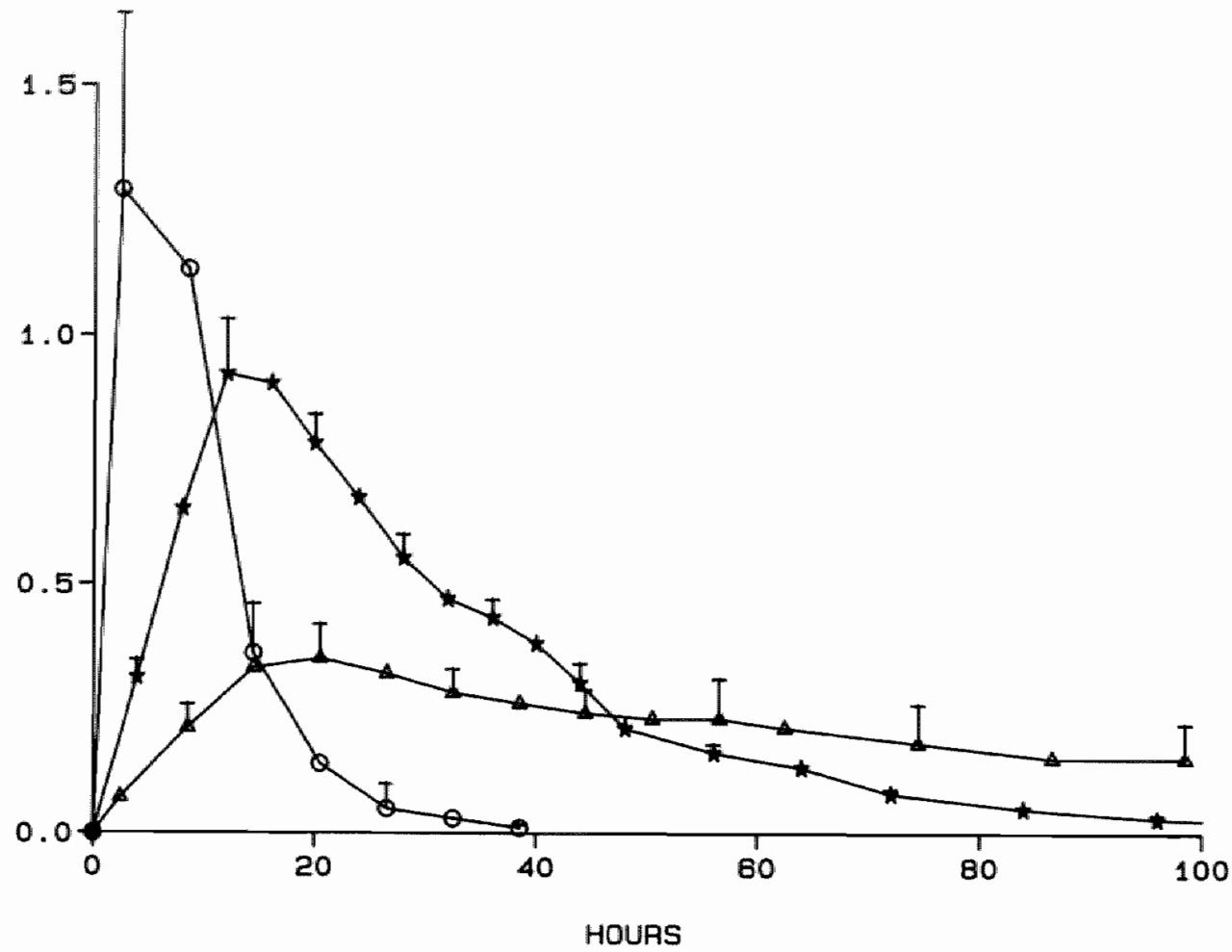

Fig. 5.12 Time course of myocardial damage after cardiac surgery $(0$, group B) and after AMI ( $\star$. The time course of skeletal mucle damage in group $B$ is also show $(\Delta)$.

Fig. 5.12 shows the time course of perioperative myocardial and skeletal muscle damage. After AMI, the release of myocardial enzymes starts later and continues for a longer period of time than after cardiac surgery. Release of enzymes from skeletal muscle is gradual and long-lasting.

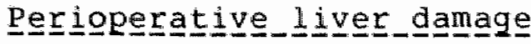

Fig. 5.13 shows that release of ALT after AMI can be complete- 

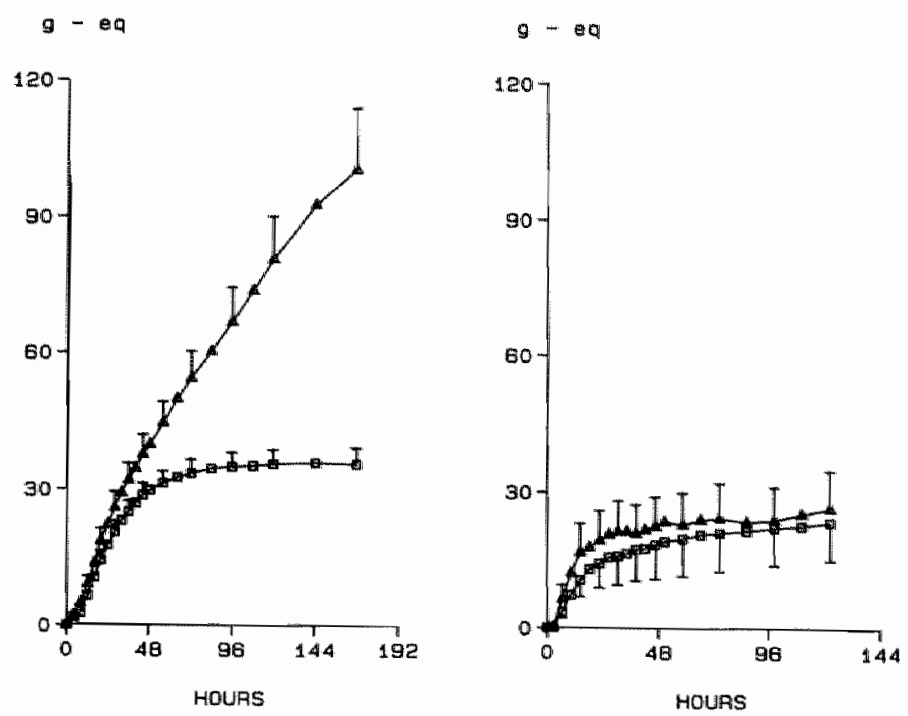

Pig. 5.13 Cumulative reiease of $H B D(\square)$ and $A L T$ ( $\triangle$ ), expreseed in g-eq of myocardium, after AMI (Left panez) and after cardiac burgery (group $B$; might panel). Additional release of ALT, indicating liver damage, is apparent after AMI.

Iy explained by myocardial celease up to about 36 hours after the onset of symptoms. Thereafter, extra release of ALT occurs, indicating liver damage. It should be noted that Fig. 5.13 is expressed in gram-equivalents of myocardium. As the liver contains approximately 8 times more $A L_{1} T$ than the heart, only a minor liver damage after AMI is indicated. It is also apparent from this figure that there is no indication of liver damage after cardiac surgery.

\section{Accuracy_in the_estimation_of_myocaroial_damage}

It has been argued that a low value of FCR improves the accuracy of calculated cumulative enzyme release after AMI [Willems, 1982; Van der Laarse, 19841. The unknown individual variation in FCR is much larger than the error in the assay of plasma enzyme 
activities. This implies that, unless a slowly catabolized enzyme is used, the error in the estimated quantity of catabolized enzyme soon becomes dominant. This argument cannot be directly applied to the situation after cardiac surgery. In that case, the unknown individual variation in plasma volume and in transcapillary protein escape may also introduce considerable error. In fact, the situation could be reversed because an uncertain value of TER is relatively unimportant if FCR is large, i.e. for a rapidly catabolized enzyme.

In order to evaluate these effects, the individual variation in circulatory paraneters is accounted for in Table 5.2 by using random fluctuations of $20 \%$ around the mean parameter values. The effect of sampling error is simulated by adding a $6 \%$ random error to the plasma activities. The combined effect of these sources of error is shown as the random error in Table 5.2. The systematic error in this Table refers to the effect of postoperative changes in plasma volume and in TER. Data of group $B$ were chosen because of the large range of myocardial injury observed in this group. Changes in TER do indeed affect the systematic error for HBD more than for CK-MB. For large injuries, the use of HBD is preferred because of the much smaller random error. This has also been demonstrated for patients with AMI [Willems, 1982, Van der Laarse, 1984]. For an intermediate extent of damage, the error for both enzymes is comparable in absolute magnitude but the systematic error observed for $\mathrm{ABD}$ will usually be preferred to the random error observed for CK-MB. A very large sytematic error is shown for HBD in case of small injuries. This is caused by the release of HBD due to hemolysis which in that case even exceeds myocardial release of $\mathrm{HBD}$. If the largely expanded plasma volume ater CPB is not taken into account, the fraction of HBD due to hemolysis is seriously underestimated and myocardial release is overestimated accordingly. Table 5.2 also shows that the random error for HBD increases for smaller injuries. This effect is partly due to the high normal steady state activity of $\mathrm{HBD} / \mathrm{C}_{\mathbf{s}}=$ $80 \mathrm{U} / 1)$, which makes the individual variation of 208 in $\mathrm{C}_{\mathrm{s}}$ important in case of a limited release, and partly due to the elevation of HBD activities due to hemolysis, which makes the 6 : error in enzyme activities more important for smaller injuries. 
Table 5.2 Error in estimated myocardial injury after surgery for HBD and CK-MB.

\begin{tabular}{|c|c|c|c|c|c|}
\hline $\begin{array}{l}\text { Extent } \\
\text { of damage }\end{array}$ & Enzyme & $\begin{array}{l}\mathrm{V} \text { and TER } \\
\text { corrected }\end{array}$ & $\begin{array}{l}\text { only } \mathrm{V} \\
\text { corrected }\end{array}$ & $\begin{array}{l}\text { anly TER } \\
\text { corrected }\end{array}$ & $\begin{array}{l}V \text { and TEl } \\
\text { uncorredt }\end{array}$ \\
\hline large & $\mathrm{HBD}$ & $0 \pm 9$ & $-8+11$ & $3 \pm 6$ & $-4 \pm 10$ \\
\hline$(40 \mathrm{~g}-\mathrm{eq})$ & $\mathrm{CK}-\mathrm{MB}$ & $0+21$ & $-2+21$ & $-8+20$ & $-10+21$ \\
\hline medium & $\mathrm{HBD}$ & $0 \pm 15$ & $-13+19$ & $30 \pm 11$ & $21 \pm 13$ \\
\hline$(8.4 g-e q)$ & $\mathrm{CK}-\mathrm{MB}$ & $0+23$ & $-2+23$ & $-7+22$ & $-9+22$ \\
\hline $\operatorname{mall}$ & $\mathrm{HBD}$ & $0+29$ & $-27+39$ & $69 \pm 15$ & $5 y+17$ \\
\hline$(3.5 \mathrm{~g}-\mathrm{eq})$ & $C K-M B$ & $0 \pm 23$ & $-2+24$ & $-7 \pm 22$ & $-9+22$ \\
\hline
\end{tabular}

Figures indicate mean systematic \pm random errors expressed as percentages. Corrections for perioperative changes in plasma volume (V) and transcapillary escape rate of proteln (TER) are described in the text.

Patient group $B$ wasi divided in three subgroups of 14 patients with respectively large, medium and small extent of myocardial injury. Total release over 48 hours was calculated from the averaged time-activity curves of each group. Systematic exror is expressed relative to the values obtained after correction of both $V$ and TER. Random error was obtained from 100 repeated calculations after adiling random varlations of 208 to the parameter values of $F C R, T E R, E R R$ and $C S$ and after adding a 68 random error to plasma enzyme actlvities. 


\subsection{Discussion}

\section{Capillary_permeabi litty}

Volume loading during $\mathrm{CPB}$ and rapid renormalization of plasma volume in the early postoperative period, with extensive diuresis, has been observed routinely [Welbourn, 1966]. The increased transcapillary protein escape during the first Ew postoperative hours, as observed in the present study may be effected by several mechanisms. Extracorporeal circulation results in the release into circulation of many vasoactive substances [Nagaka, 1975; Davies, 1980; Haslam, 1980; Schuette, 1982] including neurohormones, kinins, histamine, serotonine, activated complement factors, fibrinopeptides, adenosine nucleotides and thromboxanes. Several of these substances have been demonstrated to increase vascular permeability. An alternative explanation is suggested by the coincidence of increased permeability with the period of plasma volume reduction. Increased transcapillary protein transport and extravascular accumulation of radiolabeled albumin has been observed after plasma volume loading in several species [Wasserman, 1952; Studer, 1973; Mullins, 1982]. These data have supported the "stretched pore" hypothesis i.e. the assumption that increased capillary pressure may widen the endothelial intercellular junctions and thereby increase capillary permeabiIity [Shirley, 1957]. This has also been observed after reperfusion of ischemic myocardium or of ischemic skeletal muscle [Joyner, 1974; Camilleri, 1976; McDonagh, 1982].

\section{Enzyme_disappearance rate}

The increased apparent disappearance rate from plasma of HBD and ALT after CPB, could also be caused by an effect of CPB on the catabolic rates (FCR) of these enzymes. However, several authors have shown that surgical interventions with drastic hemodynamic disturbances such as hepatectomy, nephrectomy, splenectomy or partial ligation of the vena cava have no influence on the values of FCR for AST, ALT, CK and LDH [Dunn, 1958; Fleisher, 1963a; Strandjord, 1959; Roberts, 1975]. Enhanced extravascular 
return of protein, $i . e$ an increased value of ERR, could also influence the apparent disappearance rate of HBD and ALT. If ERR would be significantly increased, this would reduce the overall apparent disappearance rate and the increase in TER would be underestimated accordingly. From calculations not shown in the text, however, it is concluded that an appreciable increase in the value of ERR is not compatible with the magnitude and duration of the observed post-CPB disappearance of circulating HBD.

\section{Blogd_cellis}

Thrombocytopenia or functional platelet defects due to CPB have been reported by several authors and were related to a postoperative bleeding tendency [Porter, 1968; Gomes, 1970; Mckenna, 19751. These effects were usually reversible and were interpreted as a transient intravascular aggregation following the contact of platelets with incompatible surfaces in the CPB system or with the priming fluids [Bick, 1976, woltjes, 1979]. Fram Table 5.1 it is apparent that even complete release of enzymes from platelets or leucocytes will be completely masked by the release of enzymes from a small fraction of damaged erythrocytes. The loss of erythrocytes due to CPB as calculated from release of hemoglobin and HBD in the present study only amounts to approximately $2 \%$ of total red cell mass. This effect completely vanishes in the large perioperative changes in hematocrit values observed after CPB, which explains why the doss of erythrocytes due to extracorporeal circulation was not observed in a number of the above mentioned studies.

\section{Skeletal muscle}

A surprising result apparent from Fig. 5.2 is the 1 ack of release of $\mathrm{LDH}_{5}$ after skeletal muscle damage. Together with the partial inactivation of skeletal muscle $\mathrm{CK}$, as demonstrated in the dog and indicated in patients (cF. Fig. 5.11), this finding indicates that after skeletal muscle damage circumstances are favourable for local inactivation of enzymes. The considerable time needed for the transport of enzymes from skeletal muscle to 
plasma (cf. Fig. 5.11 and Fig.5.12) may also contribute to this effect. For LDH $\mathrm{LH}_{5}$ such Local inactivation could also be facilitated by its great thermolability. Moreover, the appearance of $\mathrm{LDH}_{5}$ in plasma lay be hampered by the rapid elimination from plasma of this isoenzyme as observed in several species [Boyd, 1967; Friedel, 1976; Qureshi, 1976].

Myocárdial_aldamage

Studies on the relation between plasma enzyme activities after cardiac surgery and other indices of perioperative myocardial injury, have not produced unequivocal results. In these studies, the occurrence of myocardial infarction is defined by a reference method, mostly by electrocardiography or radionuclide scanning. Then, the sensitivity, i.e. the percentage of correctly detected infarctions, and the specificity, i.e. the percentage of correct$1 y$ detected non-infarctions, of the enzymatic method can be determined. Hultgren et al. [Hultgren, 1973] found a 70\% sensitivity and $90 \%$ specificity for $\mathrm{LDH}_{1}$ but only $51 \%$ sensitivity and $69 \%$ specificity for CK. About 95 or higher sensitivities and specificities were found for $\mathrm{LDH}_{1}$ [Codd, 1977] and for CK-MB [Baur, 1979] but other studies reported sensitivities of $60 \%$ or less, also for CK-MB [Balderman, 1980; Righetti, 1977]. Several factors may contribute to these discrepancies. Cardiac enzyme release is primarily related to the extent of irreversible myocardial injury and this may be only partially true for the reference method used in these studies, which may include transient ischemia-induced abnormalities as well. Using post mortem histological verification, Horan et al. [Horan, 1971] could only confirm 31 from 56 cases with recent myocardial infarctions diagnosed by electrocardiography. Verification with angiographically assessed contractility also indicated only 50 sensitivity for electrocardiographic detection of perioperative myocardial infarction [Shirey, 1980]. Similar discrepancies were mentioned in several recent studies [Balderman, 1980; Raabe, 1980]. Another factor contributing to these divergent results are the arbitrary threshold values used by different authors for the enzymatic discrimination between 
infarctions and non-infarctions. Largly different results can often be obtained from the same data by changing the threshold value. Also, threshold values can often not be compared due to the use of different enzyme assays. In addition, corrections for enzyme release due to hemolysis or skeletal muscle damage were absent in the studies mentioned. This could explain variable results obtained for different enzymes, or for different surgical technigues.

\section{Conclus 노은}

With respect to the accuracy of estimation of perioperative myocardial damage it is concluded that the choice of marker enzymes and correction procedures is dependent on the specific aspects to be investigated. For instance, if one aims at detecting complications of cardiac surgery, e.g. injuries exceeding 10 g-eq of myocardium, one should use a slowly eliminated enzyme like HBD and apply corrections for the transient changes in plasma volume and transcapillary protein escape. In the case of HBD, correction for peroperative hemolysis is also important. Under these conditions, the total release of enzyme can be estimated with an error of approximately 10\%. On the other hand, if one would for instance be interested in further improvement of myocardial preservation techniques for coutine bypass surgery, this would imply a further reduction of injuries of less than 2 g-eq of myocardium. A rapidly eliminated enzyme like CK-MB should then be used but the random error in the estimations will be of the order of $20 \%$. In that case, corrections for hemodyrimic changes are relatively unimportant. 


\section{REFERENCES}

Agress CM, Jacobs HJ, Glassner HF, Lederer MA, Clark WG, Wroblewski F, Kaxmen A, La Due JS: Serum transaminase levels in experimental mocardial infardtion. Circulation 11, 711-713, 1955.

Agress CM, Kim JHC: Evaluation of enzyme test in the diagnosis of heart disease. Arn J Cardiol $6,641-649,1960$.

Alper $A C$, Freeman $\mathbb{T}$, Waldenström $J$ : The metabolism of gamma globulins in myeloma and allied conditions. J Clin Invest 42, 1858-1868, 1963.

Amelung $D$ : untersuchungen zur Grösze der Eliminationsgeschwindigkeit von fermenten aus dem Kaninchenserum. Hoppe-Seylers $\mathbb{Z}$ physiol Chem $318,219-228$. 1960.

Amelung D: Enzymelimination aus dem Plasma. In: Praktische Enzymologie. Schidat FW (Ed), Bern, Huber HT, 1968, 149-162.

Andersen SB, Glemert J, Wallevik K: Gamma globulin turnover and intestinal degradation of gamma globulin in the dog. J Clin Invest $42,1873-1891$, 1963.

Andersen SB: Metabolism of human gammalobulin. Oxford, Blackwell Scientific Publ, 1964 .

Andrews DF et al.: Robust estimates of location: Survey and advances. Princeton University Press, Princeton N.J. 1972.

Ashwell G, Morell AG: The role of surface carbohydrates in the hepatic recognition and transport of circulating glycoproteins. Adv Enzymol 4. 9, 99-127, 1974.

Aukland $K$, Nicolaysen $G$ : Interstitial fluid volume; local regulatory mechanisms. Physiol Rev 61, 556-643, 1981.

Baer $H$, Blount SG: The response of the serum glutamic oxalacetic transaminase to open-heart operation. Am Heart $\mathrm{J} 60,867-878,1960$.

Balderman SC, Bhayana JN, Steinbach JJ, Zaki Masud AR, Michalek S: Perioperative myocardial infarction: A diagnostic dilema. Ann Thorac surg 30: 370377,1980 .

Bär U, Ohlendorf s: Studien zur Enzymelimination I. Halbwertzeiten einiger Zellenzyme beim Menschen. KIin Wschr 48, 776-780, 1970.

Baur HR, Steele BW, Frelmesberger KF, Gobel FL: serum myocardial creatine kinase (CK-MB) after coronary arterlal bypass surgery. Am it cardiol 44 , $679-686,1979$.

Beck JV, Arnold KJ: Parameter estimation in engineering and science. John Wiley \& Sons, New York, 1977.

Beeken WL, Volwiler W, Goldsworthy PD, Garby LE, Reynolds WE, Stoggdill R, Stemler RS: Studies of I-131-ablumin catabolism and distribution in normal young male adults. I Clin Invest 41, 1312-1333, 1962 .

Berg $A$, Keul $J$ : Serumkinetik während und nach intensiver Langzeltbelastung. Dtsch Ztschr f Sportmed 8, 12-17, 1982.

Berman M, Schoenfeld R: Invariants in experimental data on linear kinetics and the formulation of models. I Appl Physics 27, 1361-1372,1956.

Bick RL, Schmalhorst WR, Arbegast NR: Alterations of hemostasis assoclated with cardiopulmonary bypass. Thromb Res 8: 285-302, 1976.

Bleifeld W, Mathey D, Hanrath P, Buss H, Effert S: Infaret size estimated from sexial serum creatine phosphokinase in relation to left ventricle dynamjas. Circulation 55: 303-311, 1977 .

Blum $\mathrm{CB}$, Levy RiJ, Eisenberg $S$, Hall M, Goebel RH, Berman M: High dengity ilpoprotein metabolism in man. J Clin Invest 60, 795-807, 1977 .

Box GEP, Draper DR: The Bayesian estimation of common parameters from several responses. Biometrika $52,355-365,1965$. 
Boyd JW: The rates of disappearance of L-lactate dehydrogenase isoenzymes from plasma. Blochlm Blophys Acta 132: 221, 1967.

Brown FF' Compartinental system analysis: State of art. IEEE Trans Biomed Eng BME $-27,1-11,1980$

Bxidigan , Schnelder KW: KInische Untersuchungen zux Pathogenese der Fermentaktivitätserhöhungen der Glutaminsure - Oxal essigsäure Trausaminase and Aldolase Jn Serum. Dtech Arch klin Med 206, 451-458, 1960.

Bueding $\mathrm{E}$, Mackinon JA: Studies of phosphoglucose isomerase of schistosoma mansoni. J Biol Chem $215,507,1955$.

Cairns JA, MLssixilis E, Fallen EL: Myocardial infarction size from serial CPK: variability of CPK gerum entry ratio with size and model of infarction. Circulation $58,1143-1153,1978$.

Camilleri JP, Joseph D, Fabiani JN, Deloche A, Schlumberger M, Relland J, Carpentiex A: Microcirculatory changes following early reperfusion in experimental myociardial infarction. Virchow Arch (Pathol Anat) 369: 315, 1976.

Clark GI, Roblson AK, Gnepp DR, Roberts $R$, Sobel BE: Effects of lymphatic transport of enzyme on plasma creatine kinase time-activity curves after myocardial infarction in dogs. Circ Res $43,162-169,1978$.

Clubb JS, Neale $F C$, Posen 5 ; The behavior of infused human placental alkaline phosphatase in human subjects. J Lab Clin Med 66, 493-507, 1965.

Codd JE, Sullivan RG, Weing RD, Barrer HB, Kaisex GC, Wlllman VL: Myocardial Injury following myocardial revascularization. Detection by isoenzyme analysi.g. Circulation 56, suppl II, 49-53, 1977.

Cohen $S$, Freeman $T$ : Metabolic heterogreneity of human gamma-globulin. Biochem I $76,475-487,1960$.

Coheñ 5 , Freeman T, McFarlane AS: Metabolism of $1-131$ labeled human albumin. C1in ScI 20, 161-170, 1961 .

Collen D, TYtgat GN, Claeys $H$, Piessens $R$ : Metabolism and distribution of fibrinogen. I. Fibrinogen turnover in physiological conditions in humans. Br J Haematol 22, 681-700, 1972a.

Collen D, Tytgat $\bar{G}_{*}$ Claeys $H$, Vexstraete M, Wallén P: Metabolism of plasminogen in healthy subjects; Effect of tranexamic acid. J Clin Invest 51, 1310$1318,1972 \mathrm{~b}$.

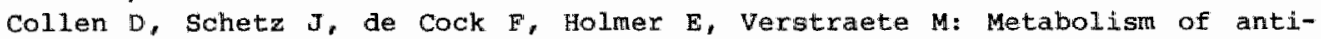
thrombin III (heparin cofactor) in man - effects of venous thrombosis and of heparin administration. Eur J Clin Invest $\underline{7}, 27-35,1977$.

Coward WA, Sawyer MB: Whole-body albumin mass and distribution in rats fed on 1ow protein diets. Br J Wutr $37,127-134,1977$.

Cutler DJ: Llnear systems analysis in pharmacokinetics. I Pharmacokin Biopharm 6. $265-282,1978$.

Davids HA, Hermens WT, Hollaar $I_{\lrcorner}$, Van der Laarse A, Huysmans HA: Extent of myocardial damage after open-heart surgery assessed from sexial plasma enzyme levels in either of two pexiods $(1975-1980)$. Br Heart J 47, 167-172, 198.2 .

Davies GC, Sobel M, Salzman EW: Elevated plasma fibrinopeptide A and thromboxane $\mathrm{B}_{2}$ levels auring cardiopulmonary bypass. Circulation 61: 808-814, 1980 .

Davies $B$, Dagget $A$, Watt DAL: serum creatine kinase and isoenzymes responses of veteran class running. Eur J Appl Physiol 48, 345-354, 1982.

Dawson DM: Efflux of enzymes fxom chloken muscile. Biochin Biophys Acta 113 , $144-157,1966$.

Dawson DM, Alper CA, Seidman J, Mendelsohn J: Measurement of serum turnover rates. Ann Int Med $70,799-805,1969$.

Delforge $J$ : Necessary and sufflcient structural condition for Iocal indentiflability of a system with linear compartments. Math Biosc 54, 159-180, 1981. 
Dewry WC, Hunter JD: Inhomogeneity of Lodated human serum albuin as detected with rat liver. I Appl Physiol 15, 961-962, 1960.

Dieter RA, Neville WE, Plfarré R: Serum enzyme alterations after open-heart surgery. Surgery 66: $328-337,1969$.

Dreyfuss $J-C$, Schapira $G$, Resnais $J$, Scebat $L$ : La creatine-kinase serique dans le diagnostic de $1^{\prime}$ infarctus myocardique. Rev Franc Etud clin Blo1 5 , 386387,1960 .

Donato I: Round table on applicetions of tracer theory to proteln turnover studies. In: Labeled proteins in tracer studies. I Donato, Gilhaud, I Sirchis (eds), Euratom, Brussels, 1966, 375-408.

Dunn $M$, Martins J, Reissmann $K R$ : The disappearance rate of glutamic transaminase from the circulation and its distribution in the body's fluid compartments and secretions. J Lab Clin Med 51, 259-265, 1958.

Erhardt LR: Clinical and pathological observations in different types of acute myocardial infarction. (A study of 84 patients decreased in a coronary care unit). Acta Med scand $560,1-78,1974$.

Fleisher GA, wakim $\mathrm{KG}$ : The fate of enzymes in body fluids - an experimental. study I. Disappearance rates of glutamic - pyruvic transaminase under various conditions. J Lab clin Med 61, 76-85, 1963a.

Fleisher GA, wakim KG: The fate of enzymes in body fluids - an experimental study III. Disappearance rates of glutamic-oxalacetic transaminase II under various conditions. I Lab Clin Med 61, 98-106, $1963 \mathrm{~b}$.

Fleisher GA, wakim KG: The role of the small intestine in the alsposal of enzymes from blood. Enzym Biol Clin 9, 81-96, 1968.

Freeman $T$, Gordon $A H$, Humphrey $J H$ : Distinction between catabolism of native and denatured proteins by the isolated perfused liver after carbon loading. Br I Exptl Pathol 39, 459-471, 1958.

Freeman $\mathrm{T}$ : The biologlcal behaviour of normal and denatured human plasma albumin. Clin Chim Acta 4, 788-792, 1959a.

Freeman $T$, Matthews CME, MCFarlane AS, Bennhold $H$, Kallee E: Albumin labelled with Iodine-131 in an analbuminaemic subject. Mature 183, 606, $1959 \mathrm{~b}$.

Freeman T: Gamma globulin metabolism in normal humans and in patients. Ser Hemat $4,76-86,1965$.

Friedel $R$, Bode $R$, Trautschold J: Vertellung heterologer, homologer und autologer Enzyme nach intravenöser Injektion. Vertellung und Transport von zellenzymen irm extrazeliuláren Raum, IIT. Mitteilung. J clin Chem clin Biachem 14, 129-136, 1976.

Gebhard MM, Denkhaus $H$, Saka $1 \mathrm{~K}$, Spleckermann $\mathrm{PG}$ : Energy metabolism and enzyme release. J Mol Med 2: 271-283, 1977.

Gervin $C A$, Hacke1 DB, Roe CR: Enzyme content of canine caralac lymph aluring acute myocardial infarction. Surgical Forum, XXV, 17-176, 1974.

Gitlin D: Distribution dynamics of circulating and extravascular I-131 plama proteins. Ann New York Acad sci 70, 122-136, 1957.

Gomes MMR, MCGoon DC: Bleeding patterns after open heart surgery. J Thorac Cardiovasc surg 60: 87-97, 1970.

Grace $A M$, Perryman $\overline{M B}$, Roberts $R$ : Purlfication and characterization of human mitochondrial creatine kinase. J Biol Chem 258: 15346-15354, 1983 .

Grande P, Fischer Hausen B, Christiansen C, Naestaft J: Estlmation of acute myocardial infarct size in man by serum $C K-M B$ measurements. Circulation 65 , $756-764,1982$.

Grotte G: Passage of dextran molecules across the blood-lymph barrier. Acta Chir Scana Suppl $211,1-84,1956$.

Hackel DB, Reimex KA, Ideker RE et al: Comparison of enzymatic and anatonic estimates of myocardial infarct size in man. Circulation 70, 824-835, 1984.

Hall CL, Hardwicke J: Low molecular weight proteinuria. Ann Rev Med 30, 199$211,1979$. 
Hansen KAI, Bjerre-Knudsen $J_{,}$Brodthagen $U$, Jordal $\mathbb{R}$, Paulev P-F: Muscle cell leakage due to long aistance training. Eur $J$ appl Physiol 48, 177-185, 1982.

Hansgon HE: Right duct lymph auring and after open heart surgery. Scand $J$ Thoracic Cardloyasc surg 9, 229-239, 1975.

Hasiam PL, Towsend PJ, Branthwaite: Conplement activation during cardiopulmonary bypass. Anaesthesia $35: 22-26,1980$.

Hauss W, Gerlach $W$, Schurmeyer $\mathbb{E}$ : Uber die Pathogenese und die klinische Bedeutung der Hyperfermentämie. Dtgch Med wochenschr 83: $1310,1958$.

Hearse DJ, Humphrey SM, Chain EB; Abrupt reoxygenation of the anoxic potassium - arrested perfused rat heart; a study of myocardial enzyme release. J Mol Cell Cardiol $5,395-407,1973$.

Hearse DJ: Cellular damage during myocardial ischemia; metabolic changes leading to enzyme leakage. In: Enzymes in cardiology. DJ Hearse, J de Leiris (eds), John wiley and Sons, London, 1979,1-19.

Hebert LA, Stuart KA, AlIhiser $C$, Rodey GR: Measurement of the fractional uptake of macromolecules by the renal vascular bed compared to other vascular beds. I Lab Clin Med 87, 716-724, 1976.

Hemker HC, Hitteveen SAGJ, Hermens Wh, Hollaar L: quantitation of the size of a myocardial infarction by determination of plasma enzymes *

In: Quantitation in cardiology, HA Snellen, HC Hemker, PG Hugenholtz, JH van Bemuel (eds), Leiden, Univ Press, 1972.

Hermens WT, Witteveen SAGJ, Hollaar $L$, Hemker HC: Effect of a thrombolytic agent (urokinase) on necrosis after acute myocardial infarction. In: Recent advances in studies on cardiac structure and metabolism PE ROY, G Rona (eds), Baltimore, Univ Park Press, 1975, vol. 10,319-329.

Hermens WTh, Willems GM, Visser MP: Quantification of circulating protelns. The Hague, Martinus Nijhoff Publ, 1982.

Hirzel HO, Sonnenblick EH, Kirk ES: Absence of a lateral border zone of intermediate creatine phosphokinase depletion surrounding a central infarct 24 hours after acute coronary occlusion in the dog. Circ Res 41, 673-683, 1977.

Hollaar $I$, Van der Laarse A: Interference of the measurement of lactate dehydrogenase (LDH) activity In human serum and plasma by LDH from blood cells. Cl.in Chim Acta 99, 135-142, 1979.

Horan LG, Flowers VC, Johnson JC: Significance of the diagnostic Q-wave of myocardial infarction. Circulation 43: $428,4971$.

Huber PJ: Robust statistics. John WLleY Sons, New York, 1981.

Hultgren $\mathrm{HN}$, Miyagrawa $M$, Buch $W$, Angell WW: Ischemic myocardial injury during cardiopulmonary bypass surgery. Am Heart J 85: 167-176, 1973.

Inoue $J$, Meda $w$, Kobatake $\Psi$ : structure of exitable membranes formed on the surface of protoplasnic drops isolated from Nitella. Biochim Biophys Acta (blomembranes) 298, 653-663, 1973 .

Inoue $M$, Hori $M$, Wishimoto $Y$, Fukui $S$, Abe H, Wada $F$, Minamino $T$ : Immunological determination of serum m-MST activity in patients with acute myocardial infarction. Br Heart is $40,1251-1256,1978$.

Jacquez $J A$ : Compartmental analysis in biology and medicine. Elsevier, Amsterdam, 1972.

Tewell WR, Krishnan C, Schloerb PR: Apparent cellular ingress of albumin in walker 256 tumor and rat muscle. Cancer Res 35, 405-408, 1975.

Johnson RN, Sammel NL, Norris RM: Depletion of myocaraial creatine kinase, lactate dehydrogenase, myoglobin and $\mathrm{k}+$ after coronary artery ligation in dogs. Cardiowasc Res 15,529-537, 1981.

Jones EA, Vergalla J, steer CJ, Bradley-Moore PR, Vierling JM: Metabolism of intact and desialylated alpha 1-antitrypsin. Clin 5ci Mol Med 55, 139-148, 1978. 
Joyner WL, Carter $\mathrm{RD}$, Raizes GS, Renkin EM: Influence of histamine and some other substances in blood-lymph transport of plasma protein and dextran in dog paw. Microvasc Res 7: 19, 1974.

Kahn JC, Gueret P, Menier $\bar{R}$, Giraudt P, Ben Farhat M, Bourdarias JP: Prognostic value of enzymatic (CPK) estimation of infarct size. J Mol Med 2, 223231, 1977 .

Karmen A: A note on the spectrophotometric assay of glutamic-oxaletic transaminase in human blood serum. J Clin Invest 34, 131-133, 1955.

Katz JH: Iron and protein kinetics studied by means of doubly labeled human crystalline transferrin. I Clin Invest $40,2143-2152,1961$.

Katz J, Bonorris G, Golden S, Sellers AL, Extravascular albumln mass and exchange in rat tissues. Clin Sci 39, 705-724, 1970a.

Katz J, Bonorris G, Sellers AL: Extravascular albumin in human tissues. Clin Sci $39,725-729,1970 \mathrm{~b}$.

Kedem 0 , Katcholsky A: Permeability of composite membranes, parts $1,2,3$. Trans Faraday Soc 59, 1918-1954, 1963.

Kedem $O$, Essig $A$ : Isotope flows and flux ratios in biological membranes. I Gen Physiol 48, 1047-1070, 1965.

Kendal MG, Stuart $A$ : The advanced theory of statistics. Griffin, London, 1973. Kibe $O$, Nilsson NJ: Observations on the diagnostic and prognostic value of some enzyme tests in myocardial infarction. Acta Med scand 182, 597-610, 1967.

Kjekshus JK, Sobel BE: Depressed myocardial creatine phosphokinase actlvity following experimental myocardial infarction in rabbit. Circ Res $27,403-$ $414,1970$.

Kobayashi $N$, Takeda $Y$ : Effects of a large dose of oestradiol on antithrombin III metabolism in male and female dogs. Eur J Clin Invest 7, 373-381, 1977.

Kouchoukos NT, Oberman $A$, Kirklin JW, Russel RO, Karp RD, PaCliflco AD, Zorn GL: Coronary bypass surgery: Analysis of factors affecting hop ital mortality. Circulation 62 (suppl I): $84-89,1980$.

Kulpers H, Drukker J, Frederik PM, Geurten P, Kranenburg GW: Muscle degeneration after exercise in rats. Int J Sports Med 4, 45-51, 1983.

Kupper $\omega$, Bleifeld $w$ : Serum enzyme changes in patients with cardiac diseases. In: Advances in clinical enzymology. E Schmidt, Fw schmidt, I Trautschold, R Friedel (Eds), Basel, $s$ Karger, 1979.

LaDue JS, Wróblewski F, Karmen A: Serum glutamic oxaloacetic transaminase activity in human acute transmural myocardial infarction. Sclence 420 , 497-499, 1954 .

Lassen NA, Parving HH, Rossing N: Filtration as the maln mechanism of overall. transcapillary protein escape from the plasma. MLcrovagc Res $7, i-1 v, 1974$.

Lees MH, Hexr RH, Hill JD, Morgan CL, Ochsner AJ, thomas $C$, Van Flleet DL; Distribution of systemic blood flow of the rhesus monkey durling cardiopulmonary bypass. I Thorac Cardiovasc surg 61: 570-586, 1971 .

Lewallen $C G$, Berman $M$, Rall JE: Studies of Iodo-albumin metabolism $I$. A mathematical approach to the kinetics. J Clin Invest 38, 66-87, 1959a.

Lewallen $C G$, Rall $\mathfrak{J E}$, Berman $M$ : Studies of liodo-albumin metabolism II. Effects of thyroid hormone. I Clin Invest $38,88-101,1959 \mathrm{~b}$.

Malmberg P: Aspertate aminotransferase activity in dog heart lymph after myocardial infarction. Scand J Clin Lab Invest 30, 153-158, 1972.

Mahey BWJ, Rowson KEK, Parr CW, Salaman MH: Studies on the mechanism of action of Riley virus. I. Action of substances affecting the reticuloendothelial system on plasma enzyme levels in mice. J Expt1 Med 122, 967-981, 1965.

Mahey BWJ, Rowson KEK, Parx CW: Studies on the mechanism of action of Riley virus IV. The reticuloendothelial system and impalred plasma enzyme clearance in infected mice. I Expt l. Med 125, 277-288, 1967. 
Malmberg P: Enzyme composition of dog heart lymph after myocardial infarction. Upsala J Med Sc1 $78,73-77,1973$.

Massarrat 5: Verhalten und schmudrate der Glutamat-oxalacetat-Transaminase in dex Blutbahn " ges Exp Med 148, 56-71, 1968.

Matthews CME: The theory of tracer experiments with I-131 Jabeled plasma protelns. Physics Med Biol $2,36-53,1957$.

MC Dondgh PF, Goldman $N$, Laks $H$ : Transcoronary solute exchange in the beating versus fibrillating canine heart on cardiopulmonary bypass. Microcirculation 2: $45-72,1982$.

McFarlane AS: Efficient trace-labelling of proteins with Iodine. Nature 183, $53,1958$.

MCFarlane As: In Vivo behavior of I-131-fibrinogen. J Clin Invest 42, 346-361, 1963.

MCFarlane AS, Koj A: Short-term measurement of catabolic rates using iodinelabeled plasma proteins. I Clin Invest 49, 1903-1911, 1970.

McGovern JJ, Jones AR, Stelnberg AG: The hematocrit of capillary blood. New Eng I Med 253: 308, 1955.

Mckenna $R$, Bachmann $F$, Whittaker $B$, Gilson JR, Weinberg M: The hemostatio mechanism after open-heart surgery. J Thorac Cardiovasc Surg 70: 298, 1975.

Mercex. DW, Varat MA: Detection of cardiac specific creatine kinase iscenzyme in sera with normal or slightly increased total creatine kinase activity. Clin Chen 21: 1088-1092, 1975a.

Mercer DW: Sinultaneous separation of serum creatine kinase and lactate dehydrogenase isoenzymes on ionexchange chrometrography. Clin Chem 21. 1102$1106,1975 \mathrm{~b}$.

Mezger VA, Kahles $H$, Stellwaag M, Spleckermann PG: Myoglobin and enzymes: simultaneously liberated diagnostic markers of myocardial cell damage. J Mol Cell Cardiol 11, 37, 1979.

Mor In LG: Creatine kinase isoenzyme-antibody reactions in immuno inhibition and immuno-nephelometry.Clin chem 25, 1415-1419, 1979.

Morris B: The hepatic and intestinal contributions to the thoracic duct lymph. Quart J Exp Physiol 41, 318-325, 1956.

Mullins RJ, Bell. DR: Changes in interstitial volume and masses of album and IgG in rabbit skin and skeletal muscle after saline volume loading. Circ Res 51: 305-313, 1982 .

Nagaka $\mathrm{H}$, Katori $\mathrm{M}$ : Inhibition of kinin formation by a kallikxein inhibitor during extracorporeal circulation in open-heart surgery. Circulation 52: $325-332,1975$.

Wicke11 WF, Allbrltten FF: Serum trangaminase content related to tissue injuxy. Surgery 42: 240-248, 1957.

Nisgen NI, Ranlov P, weis-Fogh $J$ : Evaluation of four different serum enzymes in the Alagmosis of acute myocardial infarction. Br Heart J 27, 520-526, 1965.

Nordbeck H, Kahles H, Preusse CJ, Spieckermann PG: Enzymes in cardiac lymph and coronary blood undex normal and pathophysiological conditions. J Mol Med $2,255-263,1977$.

Nordbeck H: Iymphphyslologie des Herzens, Georg Thieme Verlag, stuttgart, 4978.

Norris RM, Whitlock RMI, Barratt-Boyes $C$, small $\mathrm{CW}$, Clinical measurernent of myocardial Infarct size. Modification of a method for the estimation of total creatine phosphokinase release after myocardial infarction. circulation $51,614-620,1975$.

Norris $\mathrm{RM}$, Howell $\mathrm{D}$, Whitlock RLM, Heng MK, Peter $T$ : Enzyme release after myocardial infarction comparison of serial serum alpha-hydroxybutyrate dehydrogenase with creatine kinase levels. Eur J Cardiol 4, 461-468, 1976. 
Worton JP: Normal-mode identifiability analysis of linear compartmental systens in linear stages.

Math Biose 50, 95-115, 1980 .

Nosslin B: Nossilin's modification of the model $C$ of Reeve and Roberts. In: Andersen SB: Metabolism of human gamu globulin. Blackwell publ, oxford 1964 .

Nosslin B: Analysis of disappearance time-curves after single injection of labelled proteins. In: Protein turnover. Clba Found symp 9 (new series). Amsterdam, Elsevier, 1973, 113-130.

Noyes WD, Garby $\mathrm{I}$ : Rate of haptoglobin in synthesis in normal man. Determinam tions by the return to normal levels following hemoglobin infusion. "Sicand J Clin Lab Invest 20, 33-38, 1967.

Nydick J, Mróblewski F, LaDue JS: Evidence for increased serum glutamic oxalacetic transaminase (SGOT) activity following graded myocardial infarots in dogs. Circulation $12,161-168,1955$.

olesen H: Turnover studies with Lodine-labeled gama-macroglobulin and albumin. Scand J Clin Lab Invest $15,497-510,1963$.

Olszewsiki WL, Loe $\mathrm{K}$, Engeset $A$ : Immune proteins and other blochemical constituents of peripheral lymph in patients with malignancy and post-licradiation 1ymphedema. Lymphology 11, 174-180, 1978.

Porter JM, silver D: Alterations in fibrinolysis and coagulation associated with cardiopulmonary bypass. J Thorac Cardiovasc surg 56: 869-878, 1968 .

Posen S: Turnover of circulating enzymes. Clin Chem 16, 71-84, 1970 .

Qureshi AR, Wilkinson $\mathrm{JH}$ : The fate of circulating lactate dehydrogenase-5 in the rabbit. Clin Sci Mol Med 50,1-14,1976.

Raabe DS, Morise A, Sbarbaro JA, Gundel WD: DLagnostic criteria for acute myocardial infarction in patients undergoing coronary artery bypass surgery. Circulation $62: 869-876,1980$.

Rapaport $\mathbb{E}$ : The fractional disappearance rate of the separate isoenzymes of creatine phosphokinase in the dog. Cardiovasc Res 9, 473-477, 1975.

Reeve ${ }_{13} B$, Bailey HR: Mathematical models describing the distribution of I- ${ }^{131}$-albumin in man. J Lab Clin Med $60,923-943,1962$.

Reimer KA, Jennings $\mathrm{RB}$ : The changing amatomic reference base of evolving myocardial Infarction. Circulation 60, 866-876, 1979.

Reimer $\mathrm{KA}$, Hackel DB, Ideker RE, Mikat EM, Hartwell TD, Parker CB, Braunwald EB, Gold HK, Raabe DS, Rude RE, Sobel BE, Stone PE, Robertis R: Comparlson of enzymatic and anatomic estimates of infaret size in man. Clin Res 31: $214 \mathrm{~A}, 1983$.

Renkin BM: Multiple pathways of capillary permeabildty. Circ Res 41, 735-743, 1977.

Renkin EM, Curry FE: Transport of water and solutes across capillary endothelium. In: Membrane transport in biology. G Glebisch, DC Tosteson, HH Ussing (Eds), Berlin, Springex Verlag, 1979, vol IV A, 1-45.

Reuge $C$, Blatrix C, Brevet JP, Steinbruch M: Etude de la demie-vie de $I^{\prime \prime}$ alpht 2-macroglobuline. In: Labelied proteins in tracer studles. I Donato, $G$ Milhaud, J Sirchis (Eds) Brusselis, Euratom 1966, 143-149.

Righetti A, Crawford MH, O'Rourke RA, Haxdarson $T$, Schelbert $H, D a 11 y$ PO, De Luca M, Ashburn W, Ross J: Detection of perioperative myocaralal damage aftex coronary artery bypass graft surgery. Clxculation 55: 173-178, 1977.

Rippe B, Kamiya A, Folkow B: Transcapillary passage of albumin, effects of tissue cooling and of increases in filtration and plasma colloid osmotic pressure. Aota Physiol scand 105, 171-187, 1979.

Roberts $R$, Henry $P D$, Sobel BE: $\overline{A n}$ improved basis for enzymatic estlmation of infarct size. Circulation 52, 743-754, 1975. 
Roberts R, Sobel BE: Effect of selected drugs and myocaxdial infarction on the disappearance of creatine kinage from the circulation in conscious dogs. Cardiovas Res $11,103-112,1977$.

Robison AK, Gnepp $\overline{D R}$, Sobel BE: Imactivation of CPK in lymph. Circulation 52 , SuppI II, 5, 1975.

Rosalkid SP, Milkingon JH: Reduction of alpha-ketobutyrate by human serum. Nature 188: $1110-1111,1960$.

Rosalki 53 : serum alpha-hydroxybutyrate dehydrogenase; a new test for myocardial Infaxction. Er Heart J 25, 795-802, 1963.

Rosalki SB: An inproved procedure for serum creatine phosphokinase determinathor. I Lab Clin Med 69: 696-705, 1967.

Rossing $\mathrm{N}$, Jensen $\mathrm{H}$ : Metabolic studies of different albumin preparations. Clin Sci $32,89-99,1967$.

Rouvier J, Collen D, Swart ACW, Verstraete M: Prothrombin metabolism in healthy subjects and in two patients with congenital hypoprothrombin and related coagulation factors. HC Hemker, JJ Veltkamp (Eds). Leiden, Univ Press, $1975,167-190$.

Ruegregger P, Nydick J, Freiman A, LaDue JS: Serum activitty patterns of glutamic oxaloacetic transaminase, glutamic pyruvic transaminase and lactic dehydrogenase following graded myocardial infarction in dogs. Circ Res 7 , $4-10,1959$.

Rutili G, Arfors $\mathrm{KE}$ : Protein concentration in interstitial and lymphatic fluids from the subcutaneous tissue. Acta Physiol scand 99, 1-8, 1977 .

Saln JI, Cobb FR, McHale PA, Roe CR: Non-linear relationship between creatine kinage estimates and the histologic extent of infarction in conscious dogs: effects of reglonal myocardlal blood flow. Circulation 62, 1239-1247, 1980.

Sampson EJ, Miller AS, MCKneally SS, Whitner VS, Hannon WH, Burtic CA: Columnchromatographic separation of isoenzymes of aspartate aminotransferase. Cllin. Chem. 24: 1805-1812, 1978 .

Schuette AR, Huttemeier PC, Watkins WD, zapol WM: Effect of lung thromboxane generation on reglonal blood flow during sheep bypass. Am I Physiol 242: H462-H469, 1982 .

Schultze HE, Heremans JF: Molecular blology of human proteins. Amsterdam, Elsevier Publ Cy 1966, vol I.

Seeman P: Ultrastructure of membrane lesions in immune lysis, asmotic lysis and drug-induced lysis. Fed Proc 33, 2116-2124, 1974.

Seber GAF: Linear regression analysis. John wiley \& Sons, New York, 1977.

Shaplro SS, Martinez $J *$ Human prothrombin metabolism in normal man and in hypocoagulable subjects. J Clin Invest 48, 1292-1298, 1969.

Shell WE, KJekshus JK, Sobel BE: Quantitative assessment of the extent of myocardial infarction in the consclous dog by means of analysis of serial changes in serum creatine phosphokinase activity. I clin Invest 50, 26142625, 1971 .

Shenton LR, Bowman KO: Maximum likelihood estimation in small samples. Griffin, London, 1977.

Shirey EK, proudfit WL, Sones FM: Serum enzyme and electrocardiographic changes after coronary artery surgery. Correlation with selective cine coronary arteriography and left ventriculography. Chest 57, 122-130, 1970.

Shirley HH, Wolfram CG, Wasserman K, Mayerson HS: Capillary permeability to macromolecules: stretched pore phenomenon. Am J Physiol 190: 189-193, 1957.

Sibley JA: Significance of serum aldolase levels. Ann WY Acad Sci 75, 339-348, $1958 / 1959$.

Slutsky AS: Individual values for the disappearance rate parameter ( $k$ f) in the enzymatic estimation of infarct size. Circulation $56,545-547,197 \%$.

Smith AF: Diagnostic value of serum creatine kinase in a coronary care unit. Lancet $\underline{2}, 178-182,1967$. 
Smith AF, Wong PC-P, Oliver MF: Release of mitochondrial enzymes in acute myocardial infarction. J Mol Med $2,265-269,1977$.

Sobel BE, Bresnahan GF, Shell WE, Yoder RD: Estimation of infarct size in man and its relation to prognosis. Circulation $46,640-648,1972$.

Sobel BE, Markham J, Karlsberg RP, Roberts $R$ : The nature of alsappearance of creatine kinase from the circulation and its influences on enzynatic estimation of infarct size. Circ Res $41,836-844,1977$.

Spieckermann PG, Gebhara MM, Sakai K: Influence of acid base changes on enzyme release in the isolated perfused guinea pig heart. J Mol Med $2,331-335$, $1977 a$.

Spieckermann PG, Norabeck $H$, Preusse $C J$ : From heart to plasma. In: Enzymes in cardiology. DJ Hearse, J de Leiris (eds), John Wiley and sons, New York, $1979,81-96$.

strandjord PE, Thomas KE, White LP: Studies on isocitric and lactic dehydrogenases in experimental myocardial infarction. $J$ clin Invest 38, 2111-2118, 1959.

strandjord PE, Clayson $\mathrm{KJ}$ : Diagnosis of acute myocardial infarction on basis of heat-stable serum lactic dehydrogenase. J Lab Clin Med 58, 962, 1961.

studer RK, Morgan J, Penkoske M, Potchen EJ: Regional vascular volune and extravascular accumulation of labeled protein during plasma volume expansion. Am J Physiol. 224: 699-704, 1973.

Swain JL, Cobb FR, McHale PA, Roe ChR: Nonlinear relationship between creatine kinase estimates and histologic extent of infarction in conscious dogs: Effects of regional myocardial blood flow. Circulation $62: 1239-1247,1980$.

szabó G: Movement of proteins into blood capillaries. In: Ergebnisse der Angiologie. Basic Lymphology, Földi M (Ed). Stuttgart, Fk Schattauer Verlag, $1976,31-50$.

Szabó G, Magyar Z: The relationship between tissue fluid and lymph II. Enzymes in tissue fluid and lymph. Lymphology 11, 101-105, 1978a.

szabó G: Enzymes in tissue fluid and peripheral 1ymph. Lymphology 11, 147-155. $1978 \mathrm{~b}$.

Takeda $Y$, Reeve EB: Distribution and excretion of I-131-iodide in man on pharmacologic doses of lodides. J Lab CIin Med 60, 944-953, 1962.

Takeda $Y$, Reeve EB: Studies of the metabolism and distribution of albumin with autologous I-131-albumin in healthy men. J Iab Clin Med 61, 183-202, 1963.

Takeda $Y$ : Studies on the metabolism and distribution of fibrinogen in healthy men with autologous I-125-labeliled fibrinogen. J clin Invest 45, 103-111, 1966.

Takeda $Y$ : Studies of the metabolism and distribution of prothrombin in healthy men with homologous I-125-prothrombin. Thromb Diath Haemorrh 27, 472-489, 1972.

Takeda $Y$, Takeuchi T: studies of fibrinogen metabolism in healthy and hypertensive female subjects with the use of autologous $1-125$-fibrinogen. Thromb Haemostas (stuttg) 39, 39-45, 1978 .

Taylor $A E$, Granger $D N$, Brace $\overrightarrow{R A}$ : Analysis of lymphatic flux data $I$. Estimation of the reflection coefficient and permeablilty-surface area product for total protein. Microvasc Res 13, 297-313, 1977.

Taylor AE: Capillary tuld filtration. Starling forces and lymph flow. Circ Res: $49,557-575,1981$.

Teorell $T$ : Kinetics of distribution of substances administered to the body. I The extravascular modes of administration. Arch Int pharmacodyn Ther 57 , $205-240,1937$.

Thompson PL, F'letcher EE, Katavatis V: Enzymatic indices of myocardial necrosis; Influence on short- and long-term prognosis after myocardial infarction. Circulation 59, 113-119, 1979. 
gommalni $G$, Presta M: Prediction of infarct size by enzymatic techniques; modification of a method and clinical application. Br heart $J 42,326-332$, 1979.

Irautschold I, Scheitzer G: Enzyme im Serum bei Herzerkrankungen. In: Praktigche Enzymologie. FW Schmidt (Ed), Bern, Huber, 1968.

Van Beaunont Rochelle RH: Exythrocyte volume stability with plasma osmolarity changes in exexcising man. Proc Soc Exp Biol Med 145, 240-243, 1974.

Van dex Laarse $A$, Hollaar $L$, van der Valk LJM, Witteveen SAGJ: Enzyme release from and enzyme depletion in rat heart cell eultures during anoxia. J Mol Med $3,123-131,1978$.

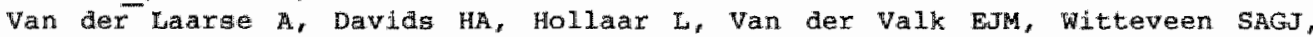
Hermens Wh: kecognition and quantification of myocardial injury by means of plasma enzyme and isoenzyme activities after cardiac surgery. Br heart $\mathrm{J}$ 41: $660,1979 a$.

Van der Laarse $A$, Hollaar $L$, Kokshoorn LJM, witteveen SAGJ: The activity of cardi-gpecific isoenzymes of creatine phosphokinase and lactate dehydrogenase in monolayer cultures of neonatal rat heart cells. J Mol Cell Cardiol 11, 501-510, 1979b.

Van der Laarse $A$, Hollaar $L$, Van der Valk: Rellease of alpha-hydroxybutyrate dehydrogenase from neonatal rat heart cell cultures exposed to anoxia and reoxygenation. Comparison with impairment of structure and function of damaged cardiac cells. Cardiovasc Res 13, 245-253, $1979 \mathrm{C}$.

Van der Laarse A: Quantification of anoxia-induced cell damage in rat heart cell cultures using enzymatic, histologic and contractile performance techniques. In: Quantification of myocardial ischemia. H Kreuzer, Ww Parmley, P Rentrop, HW Heiss (Eds). New York, Gerhard Witzstrock Publ House, 461-471, $1980 \mathrm{a}$.

Van der Luarse A, Hollaar I, Bloys van Treslong CHT: Evaluation of a columnchromatographlc and electrophoretic technique for separation of the two isoenzymes of aspartate aminotransferase (AST). Clin Chim Acta 102, 257$260,1980 \mathrm{~b}$.

Van der Laarse A, Dijkshoorn NJ, Hollaar $L$, Caspers $T$ : The (iso)-enzyme activities of lactate dehydrogenase, alpha-hydroxybutyrate dehydrogenase, creatine kinase and aspartate aminotransferase in human myocardial biopsies and autopsies. Clin Chim Acta, 104, 381-391, 1980c.

Van der Laarse A, Davids HA, Hollaar L, Hermens WT: Enhanced release of mitochondili aspartate aminotransferase (mAST) from anoxic heart cell cultures during reoxygenation. Comparison to plasma mast levels in patients after acute mocaraial infarction and after cardiac surgery. Cardiovasc Res 15 , $11-20,1981$.

Van der Laarse $A$, Hermens WTh, Hollaar $L$, Jol M, Willems GM, Liem AH, Souverifn JHM, Ouahof $\mathrm{JH}_{*}$ de Hooge J, Buis B, Arntzenius AC: Assessment of myocardial damage in patients with acute myacardial infarction by serial. measurement of serum alpha-hydroxybutyrate dehydrogenase levels. Am Heart J $107: 248-260,1984$.

Van dex Veen $\mathrm{KJ}$, Willebrands $A F$ : Isoenzymes of creatine phosphokinase in tissue extracts and in normal and pathological sera. Clin Chim Acta $13,312-$ $316,1966$.

Van Dieijen-Visser MP: Behaviour of tissue enzymes in the circulation. Thesis, Meppel, 1981.

Van Furth $R$, Cohn ZA, Hirsch JG, Humphrey JH, Spector WG, Langevoort HL: The mononuclear phagocyte system; a new classification of macrophages, monocytes and their precursor cells. Bull wld H1th org 46, 845-852, 1972 .

Van Kampen EJ, Zijlstra WG: standardization of hemoglobinometry. I The hemoglobin cyanide method. Clin Chim Acta $6: 538,1961$. 
Vatner SE, Baig $H$, Manders WT, Maroko PR: Effects of coronary artery reperfusion on myocardial infarct size calculated from creatine kinase. J clin Invest 61: 1048-1056, 1978.

Veng-Pedersen P: An algorithm and computer program for deconvolution in linear pharmacokinetics. J Pharmacokin Biophatrm $8,463-481$, 1980 .

Vincent WR, Rapaport E: Serum creatine phosphokinase in the alagnosis of acute myocardial infarction. Amer $J$ cardiol 15, 17-26, 1965.

Visser MP: The two-compartment circulatory model used for enzymatic infarct quantitation. Circ Res $42,877-879,1978$.

Visser MP, Krill MTA, Boumans M, Willems GM, Hermens WT: Selection of marker enzymes for the quantitation of tissue damage. Eur Heart $J 2$, Suppl $\mathbb{A}, 22$, 1981 a.

Visser MP, Kxill MPA, Muijtjens AMM, Willems GM, Hermens WT: Distribution of enzymes in dog heart and liver. Significance for assessment of tissue damage from data on plasma enzyme activities. Clin chem 27, 1845-1850, 1981b.

Visser MP, Krill MTA, Willems GM, Hermens WT: Selection of a sultable clrculatory model for the plasma clearance and distribution of cardiac enzymes in the dog. Cardiorasc Res 15, 35-42, 1981c.

Visser MP, Krill MTA, Willems GM, Hermens WT: Plasma volume determination by use of enzyme dilution in the dog. Lab Animals 16, 248-255, 1982 .

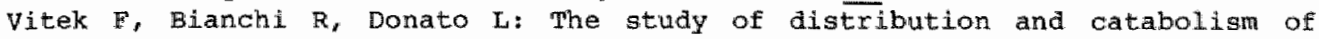
labeled serum albumin by means of analog computer technique. I Nucl Biol Med 10, 121-130, 1966.

Volk P: Myoglobin in the serum after myocardial infarction. München Med Wschr $115,2122-2128,1973$.

Wakim KG, Fleisher GA: The fate of enzymes in body fluids - an experimental study II. Disappearance rates of glutamic - oxalacetic transaminase I under various conditions. J Lab clin Med $61,86-97,1963 \mathrm{a}$.

Wakim KG, Fleisher GA: The fate of enzymes in body Eluids - an experimental study IV. Relationship of the reticulo-endothelial system to activities and disappearance rates of various enzymes. I Lab Clin Med 61, 107-119, 1963b.

Wallevik $K$ : In vivo structure and stability of serum albumin in relation to its normal catabolism. Acta Physiol scand, suppl 471, 1-56, 1979.

Walter $\mathbb{E}$, Lecourtier $Y$ : Unidentifiable compartmental models: What to do? Math Biosc $56,1-25,1981$.

Wassernan $\mathrm{K}$, Mayerson HS: Mechanism of plasma proteln changes following galine infusions. Am J Physiol 170: 1-10, 1952.

Welbourn $W$, Melrose DG, Moss DW: Changes in serum enzyme levels accompanying cardiac surgery with extracoporeal circulation. J Clin Pathol 19: 220-232, 1966.

Wetterfors J: Catabolism and distribution of serum-ablumin in the dog. An experimental study with homologous 131-I-albumin. Acta Med scand 177, 243$256,1965$.

Wiederhielm $C A$ : Dynamics of capillary fluid exchange. A nonlinear computer simulation. Microvasc Res 18, 48-82, 1979 .

willems GM, Muijtjens AMM, Lambil FHH, Hermens WT: Estimation of clrculatory parameters in patients with acute myocardial infarction. Signiflcance for calculation of enzymatic infarct size. Cardiovase Res 13, 578-587, 1979.

Willems GM, Visser MP, Krill MTA, Hermens WT: Quantitative analyals of plasma enzyme levels based upon simultaneous determination of different enzymes. Cardiovasc Res 16, 120-131, 1982.

Wissenschaftlichte Tabellen Geigy: Hämatologie und Humangenet1k, (Ciba-Gelgy) 8. Auflage, Basel, 1979.

Witteveen SAGJ, Hermens WT, Hemkex HC, Hollaar L: Quantitation of enzyme release from infarcted heart muscle. In: Ischenlc heart disease. IH de Haas, HC Hemker, HA Snellen (Eds). Leiden, Univ Press, 1970, 36-42. 
Witteveen SAGJ: Aeseagment of the extent of a mocardiall infarction on the basis of plasma enzyme levels. Thesis, Leiden, 1972.

Witteveen SAGJ, Henker HC, Hollaar L, Hermens WT: Quantitation of infarct size In man by means of plasma enzyme levels. Br Heart $\mathbb{3} 37,795-803,1975$.

wohlgemuth I: Uber eine neue Methode zur quantitativen Bestimmung des diastatischen Ferments. Biachem $z \underline{9}, 1-9,1908$.

Woltjes $J$, De Jong JCF, Ten Duis HJ, Wlilevulux ChRH: The priming of extracorporeal circuits: The effect on canine blood elements. Transfusion 19: 552$557,1979$.

Wroblewsiki F, LaDue IS: Lactic dehydrogenase activity in blood. Proc soc Exp Bilol Med 90: 210-213, 1955.

zierler KL: Muscle membrane as a dymamic structure and its permeability to aldolage. Ann NY Acad Sci 75, 227-234, 1958.

Zizza F, Cambell TJ, Reeve EB: The nature and rates of excretion of radioactive breakdown products of $I-131-a l b u m i n$ in the rabbit. J. Gen Physiol 43, 397, 1959. 


\section{CONCLUSIONS AND SUMMARY}

The plasma activities of cardiac enzymes, e.g. creatine kinase and $\alpha$-hydroxybutyrate dehydrogenase, have been used routinely during the past 25 years to estimate the extent of myocardial damage in patients with acute myocardial infarction (AMT). Yet, very few data are available concerning the kinetics of the plasma levels of these enzymes in man.

In order to determine the total quantity of enzyme released into plasma (to estimate the total loss of myocardium) from the elevated plasma activities the rate of degradation of the enzyme cicculating in plasma must be known. This rate of degradation equals the Fractional Catabolic Rate constant (FCR) multiplied by the instantaneous plasma activity.

In this thesis a method is presented to estimate the catabolic rate FCR of cardiac enzymes from simultaneously sampled timeactivity curves of several cardiac enzymes, as measured in patients with AMI. This estimation is complicated because in this situation also the input of enzyme into the plasma is unknown and must be estimated. Moreover it is also necessary to account for the distribution of enzyme between plasma and extravascular fluid. This necessitates the introduction of two additional circulatory model parameters, the Transcapillary Escape Rate constant (TER) and the Extravascular Return Rate constant (ERR) characterizing the exchange rates between these pools of enzyme. The parameters FCR, TER and ERR define a two-compartment circulatory model for the elimination and distribution of circulating proteins.

The existing data on the circulation of proteins in the body are reviewed in Chapter 2, where it is concluded that the process of elimination of protein is poorly understood, while the physiological data on extravasation and distribution of native proteins lack the accuracy required for modelling the kinetics of the plasma levels. In contrast, detailed data are available from kinetic studies were radiolabelled plasma proteins intravenously injected in man. From a review of these studies a range is derived for the values of TER and ERR of the enzymes studied in this 
thesis. Data on cellular enzyme release and on the transport of such enzymes to the circulation are reviewed in the final sectior of this chapter.

In chapter 3 it is shown that in case of a strictly parallel progressing release into plasma of several enzymes, the circulatory model parameters and the input into plasma may be estimated from the (sufficiently) sampled plasma enzyme activity curves. The release of the studied enzymes in a constant (time-independent) proportion is crucial for the validity of the proposed estimation procedure. This assumption however is supported by a large body of experimental data, cf. Chapter 2.6 .

Next, the sensitivity of the estimation procedure to the experimental error and the sampling schedule is investigated by means of simulated data. It appears that inclusion of a slowly catabo11 zed enzyme (FCR $<0.05 \mathrm{~h}^{-1}$ ) is necessary in order to attain an acceptable accuracy. Also the effect of deviations from the model assumptions upon the estimation of the circulatory model parameters is investigated, in particular the effect of non-simultaneous release and of local degradation of enzyme.

In Chapter 4 the estimation procedure is applied to the analysis of plasma activity curves of several cardiac enzymes simultaneously sampled in patients with AMI. Thus the value of FCR is determined for the enzymes $C K, C K-M B, H B D, A S T, G P I$ and ALT. The value of FCR estimated for CK is much higher than the value used in most literature for the quantification of cumulative release of CK in man. Thus total release of $C K$ calculated with this higher value of $F C R_{C K}$ is correspondingly higher. Application of these values of FCR and comparisons with published data on histological infarct size results in the conclusion that in man the larger part of enzyme originally present in the infarcted area is recovered in the plasma. Furthermore the results presented in this chapter indicate that cytoplasmic enzymes are released into plasma in quantities proportional to heart tissue enzyme content.

This finding is used in chapter 5 in the analysis of plasma enzyme activities measured in patients after open heart surgery. The interpretation of plasma enzyme activities in this group of patients is complicated by enzyme release from damaged skeletal 
muscle and from erythrocytes in addition to cardiac enzyme release. It also appears that in these patients the plasma volume is considerably expanded immediately after coronary pulmonary bypass. In a postoperative period of approximately eight hours the plasma volume renormalizes. This period of plasma volume contraction is accompanied by an increased value of TER. Such transient hemodynamic disturbances must be accounted for in the calculation of cumulative enzyme release. It is shown in chapter 5 that the contributions of damaged heart tissue, skeletal muscle and blood cells may be discriminated. An analysis of the effects of various sources of error upon the calculation of cumulative enzyme release is also presented in this chapter. This error analysis demonstrates that HBD should be used for the quantification of heart damage if the anticipated extent of damage is large. However, it is concluded that in case of minor heart injury $\mathrm{CK}-\mathrm{MB}$ permits more accurate estimation. 



\section{CONCLUSIES EN SAMENVATTING}

De verhoging van plasma activiteiten vam caraiale enzymen. zoals creatine kinase en a-hydroxybutyrate dehydrogenase, worden reeds meer dan 25 jaar gebruikt woor het schaten van de hartschade in patiënten met een acuut myocardinfarct. Toch is er nog weinig bekend over de kwantitatieve beschrijving van de kinetiek van de plasma spiegels van deze enzymen in de mens.

om met behulp van de gestegen plasma enzym activiteiten de totale hartschade te kunnen schatten is het nodig de afbraaksnelheid van deze enzymen uit plasma te kennen. Deze snelheid is gelijk aan de "Fractional catabolic Rate constant" (FCR) vermenigvuldigd met de plasma-activiteit.

In dit proefschrift wordt een methode beschreven om deze afbraak constante FCR te schatten wit de gelijktijdige verhoogde activiteitscurves van verscheidene cardiale enzymen, zoals die gemeten worden in plasma van patiënten met een acuut myocard infarct. Deze schatting is gecompliceerd door de omstandigheid dat de instroom van enzym in plasma in dergelijke gevallen onbekend is. Daarnaast blijkt het nodig te zijn on rekening te houden met de verdeling van enzym over het plasma volume en een extravasculair vloeistofvolume. Dit laatste vereist de invoering van twee extra parameters, namelijk een constante TER (Transcapillary Escape Rate constant) om de ontsnappingssnelheid van enzym vanuit het plasma naar de extravasculaire vloeistof te karakteriseren en de parameter ERR (Extravascular Return Rate constant) die de snelheid van transport van enzym vanuit het extravasculaire volume terug naar het plasma aangeeft. Deze drie model parameters FCR, TER en ERR definiëren het twee-compartimenten model voor de circulatie van eiwitten in het lichaam.

Van de bestaande gegevens over de circulatie van eiwitien in het lichaam wordt in Hoofdstuk 2 een overzicht gegeven. Hieruit wordt de conclusie getrokken dat weinig inzicht bestaat in het mechanisme van de eliminatie van eiwitten uit de circulatie terwijl de fysiologische gegevens betreffende extravasatie en verdeling van de normal voorkomende eiwitten niet voldoende nauwkeurig zijn om te gebruiken bij het modelleren van de kine- 
tiek van plasma spiegels. Er zijn echter wel veel gegevens over de circulatie van eiwitten bekend uit studies waarbij radioactief gelabelde plasma eiwitten zijn ingespoten in de mens. Uit een overzicht van deze onderzoekingen worden grenzen afgeleid voor de warden van TER en ERR voor de enzymen van belang in dit proefschrift. Tenslotte worden in dit hoofdstuk de gegevens besproken die bekend zijn over cellulaire enzymutstorting en over het transport van uitgestorte enzymen naar het plasma.

In Hoofdstuk 3 wordt aangetoond dat bij gelijktijdig (parallel) verlopende uitstorting in het plasma van de verschillende enzymen de circulatoire modelparameters FCR, TER en ERR, em de uitstortingsfunctie geschat kunnen worden uit de (voldoende Erequent) bemonsterde activiteitscurves. Hierbij is curcial dat de bestudeerde enzymen in een (tijdsonafhankelijke) constante verhouding uitgestort worden. Deze veronderstelling wordt, zoals in Hoofdstuk 2 van dit proefschrift beschreven is, echter door een veelvoud van experimentele gegevens aannemelijk gemaakt. Voorts wordt in Hoofdstuk 3 met behulp van gesimuleerde data de gevoeligheid van de schattingsprocedure voor experimentele fouten onderzocht. Hierbij blijkt het, voor het bereiken van een acceptabele nauwkeurigheid, noodzakelijk te zijn dat een van de in de analyse betrokken enzymen langzaam afgebroken wordt (FCR $<.05$ $\mathrm{h}^{-1}$ ). Ook wordt het effect geëvalueerd van afwijking van het model op de schatting van de circulatoire modelparameters, zoals niet-gelijktijige uitstorting of lokale denaturatie van een van de enzymen.

In Moofdstuk 4 wordt de ontwikkelde schattingsmethode toegepast op de plasma-activiteit curves van verscheidene cardiale enzymen die gel:jktijdig bemonsterd zijn in patiënten met een acuut myocardinfarct. Hierbij is de waarde van FCR (de afbraakconstante) bepald voor de enzymen CK, CK-MB, HBD, AST, GPI en ALT. De gevonden waarde voor $\mathrm{FCR}_{\mathrm{CK}}$ blijkt hoger te zijn dan de warden die in de meeste literatuur wordt gebruikt voor het quantificeren van de totale $C K$ uitstorting. De met deze (hogere) warde van FCR berekende cumulatieve uitstorting is dan ook evenredig groter. 
Vergelijking wan de zo berekende enzymuitstorting met gepubliceerde gegevens over histologlsch geschatte infarctgroottes leidt tot de conclusie dat na een acuut myocardinfarct de enzymen die oorspronkelijk in het beschadigde weefsel aanwezig zijn grotendeels in de cicculatie terecht komen. Voorts wordt in Hoofdstuk 4 geconcludeerd dat cytoplasmatische cardiale enzymen bij een hartinfarct in de circulatie uitgestort worden in de verhouding warin deze enzymen oorspronkelijk in het hartweetsel aanwezig zijn.

Deze bevinding wordt in Hoofdstuk 5 toegepast bij de analyse van de plasma enzyme-activiteiten van patiènten na open hart chirurgie. Bij de interpretatie van de stijging van plasma activiteiten van enzymen in deze patiënten moet rekening gehouden worden met enzymuitstorting ten gevolge van de beschadiging van skeletspier en van (rode) bloedcellen. Daarnaast blijkt in deze patiënten na de aansluiting op de hart-long machine het plasma volume aanzienlijk geëxpandeerd te zijn. In een periode van ongeveer acht uur na de operatie daalt het plasma volume weer tot de normale warde. Deze plasmavolume daling gaat vergezeld van een verhoogd transcapillair transport van eiwitten uit het plasma naar het extravasculaire volume.

In Hoofdstuk 5 wordt beschreven hoe de bijdragen van beschadigde skeletspier, bloedeellen en hartspier onderscheiden kunnen worden. Tenslotte wordt in dit hoofdstuk een analyse gepresenteerd van de effecten van verschillende foutenbronnen op de becekening van de cumulatieve enzynuitstorting. Hieruit wordt geconcludeerd dat HBD gebruikt dient te worden voor het schatten van de hartschade indien verwacht wordt dat deze aanzienlijk zal zijn, mits gecorrigeerd wordt voor peroperatieve hemolyse, terwijl CK-MB wordt aanbevolen voor het schatten van de kleinere hartschades. 


\section{ABBREVIATIONS}

ALT

AMI

AST

$A(t)$

CAST

$c_{b}(t)$

$\mathrm{CCU}$

CPB

CK

$\mathrm{CK}-\mathrm{BB}, \mathrm{MB}, \mathrm{MM}$

$\mathrm{CS}$

$\mathrm{CV}$

$E_{b}(t)$

ERR

$E(t)$

$\mathrm{FCR}$

$E(t)$

g-eq

GPI

$\mathrm{H}_{\mathrm{b}}$

$H B D$

$\mathrm{Ht}$

LDH

$\mathrm{LDH}_{1, \ldots 5}$

$M$

MAST

$P_{b}(t)$

$P(t)$

$Q(t)$

$\mathrm{R}$
Alanime aminotransferase ( $\mathrm{EC} 2.6 .1 .2$.

Acute Myocardial infacction

Aspartate aminotransferase (EC 2.6.1.1.)

Total quantity of enzyme (protein) released into plasma upto time $t$

Cytoplasmic isoenzyme of AST

Plasma concentration in response upon a unit bolus injection at $t=0$

Coronary care unit

Cardiopulmonary bypass

creatine kinase ( $\mathrm{EC} 2.7 .3 .2$.

Isoenzymes of creatine kinase

Normal steady state activity of an enzyme

Coefficient of variation

Extravascular pool of protein in respons upon a unit bolus injection at $t=0$

Extravascular return rate constant

Extravascular pool of protein

Fractional catabolic rate constant

Input of protein into the plasma per hour

Gram equivalents

Glucosephosphate isomerase (EC 5.3.1.9.)

Hemoglobin

a-hydroxybutyrate dehydrogenase

Hematocrit

Lactate dehydrogenase (EC 1.1.1.27.)

Lactate dehydrogenase isoenzymes

Relative molecular weight

Mitochondrial isoenzyme of AST

Plasma pool of protein in response upon an unit bolus injection at $t=0$

Plasma pool of protein

Cumulative enzyme release per litre of plasma upto time $t$

Correlation coefficient 
Res?

$\mathrm{SD}$

SEM

$S \mathbb{K}$

SSR

TER

U

$V(t)$
Square root of the mean squared percentual deviation between data and $E \mathbb{i t}$

Standard deviation

Standard error of the mean

Streptokinase

Sum of squared residuals

Transcapillary Escape Rate constant

Units of enzyme activity. One unit is the enzyme activity that converts one umol of substrate per minute

Plasma volume 


\section{CURRICULUM VITAE}

De schrijver van dit proefschrift is op 9 mei 1948 geboren in Utrecht. Na het behalen van het gymasium $B$ diploma aan het Nieuwe Lyceum te Bilthoven (1966) begon hij zijn studie in de wis- en natuurkunde aan de Rijksuniwersiteit Utrecht, war hij tijdens zijn doctoral studie gedurende twee jaren een studenten assistentschap vervulde bij Prof. Dr. W. Eckhaus. In juni 1970 behalde hij aldaar aan de Faculteit der Wis-en Natuurkunde met lof het doctoraal examen Wiskunde met prof. Dr. A.F. Monna en Dr. E. Bertin als afstudeerdocenten.

Hierna werkte hij tot december 1974 op de afdeling Toegepaste Wiskunde van de Stichting Mathematisch Centrum te Ansterdam. Gedurende het eerste jaar van zijn verblijf op deze afdeling werd gewerkt aan approximatietheorie en werd mede door de schrijver een colloguium over dit onderwerp georganiseerd. Vervolgens is op instigatie van het afdelingshoofd Prof. Dr. H.A. Lauwerier het werk gericht op wiskundige toepassingen in de biologie en werd de werkgroep Biomathematica gestart.

Eind 1974 trad de schrijver van dit proefschrift in dienst van de Rijksuniversiteit Limburg, waar hij sindsdien werkzaam is binnen het deelproject Myocard (deelprojectleider: Prof. Dr. R.S. Reneman) in het onderzoeksproject: "Schatting van hartspierschade en andere toepassingen van kwantificeringsmethoden voor circulerende eiwitten" (projectleider: Dr. W.Th. Hermens). Verder werkt hij mee aan het project: "Eiwit-lipide interacties bestudeerd met ellipsometrische technieken" (projectleider: Dr. P.A. Cuypers). 


\section{NAWOORD}

Het is inmiddels meer dan tien jaar geleden dat ik in de werkgroep Biomathematica van de Stichting Mathematisch centrum te Amsterdam kennismakte met het onderwerp van deze thesis via de promotor Prof. Dr. H.C. Hemker, die in 1969 op de afdeling Cariabiochemie in Leiden een studie begonnen was nax de mogelijkheden om hartschade te kwantificeren met behulp van de stijging van plasma-activiteiten van cardiale enzymen. Coen, alleen all hierom wardeer $i k$ het bijzonder dat je als promotor hebt willen optreden.

Het in dit proefschrift beschreven onderzoek is uitgevoerd aan de Rijksuniversiteit Limburg in het kader van het project: "Schatting van hartspierschade en andere toepassingen van kwantificeringsmethoden voor circulerende eiwitten" onder leiding van co-promotor Dr. W.Th. Hermens. Wim, zonder jouw voortdurende en vasthoudende belangstelling en stimulering zou dit werk nooit voltooid zijn. Daarnaast wil je danken voor de hulp die jij mij bij dit werk hebt gegeven en voor de vele verhelderende discussies die ik met je mocht voeren.

Voor het ter beschikking stellen van vele in dit proefschrift verwerkte patiëntendata dank ik: Arnoud van der Laarse en Lenie Hollaar, afdeling Cardiobiochemie, Academisch Ziekenhuis Leiden en Ger van der Vusse, capaciteitsgroep Fysiologie, Rijksuniversiteit Limburg.

De dierexperimentele gegevens zijn verzameld door Marja van Dieijen en Erik van der Veen. Vele biochemische bepalingen ten behoeve van dit onderzoek zijn verricht door Marie-Louise Boumans. Vic Dreessen heeft een belangrijke bijdrage geleverd aan het vervaardigen van de Fortran programma"s en daarnaast dank ik hem voor de produktie van de figuren in dit proefschrift. Vele discussies gevoerd met Arno Muijtjens over de in dit onderzoek gebruikte methoden hebben zeker bijgedragen om mijn gedachten te ordenen. Van de medewerkers van de Dienst Informatie Verwerking heb ik alle medewerking ondervonden, zodat het mogelijk was het excessief lange rekenwerk uit te voeren. 
Wast het vele typewerk in de voorbereidende fase heeft Rosy Hanssen met professionele hand de produktie van het (foto-reproduktie geredel manuscript verzorga. Voor de plezierige samenwerking en voor haar grote inbreng in de vormgeving bedank ik haar. 